\title{
SURFACE WATER AND ATMOSPHERIC CARBON DIOXIDE AND NITROUS OXIDE OBSERVATIONS \\ BY SHIPBOAPD AUTOMATED GAS CHROMATOGRAPHY: RESULTS FROM EXPEDITIONS BETWEEN 1977 AND 1990
}

R. F. Weiss
F. A. Van Woy
P. K. Salameh

Scripps Institution of Oceanography University of California, San Diego La Jolla, California 92093-0220

Environmental Sciences Division Publication No. 3987

Scripps Institution of Oceanography Reference 92-11

Date Published: December 1992

Prepared by R. J. Sepanski

Energy, Environment, and Resources Center

The University of Tennessee

Knoxville, Tennessee 37996

Prepared for the

Global Change Research Program

Environmental Sciences Division

Office of Health and Environmental Research

U.S. Department of Energy

Budget Activity Number KP 0500000

Prepared by the

Carbon Dioxide Information Analysis Center

Environmental Sciences Division

OAK RIDGE NATIONAL LABORATORY

Oak Ridge, Tennessee 37831-6335

managed by

MARTIN MARIETTA ENERGY SYSTEMS, INC. for the

U.S. DEPARTMENT OF ENERGY under contract DE-AC05-84OR21400 
CONTENTS

Page

LIST OF FIGURES $\ldots \ldots \ldots \ldots \ldots \ldots \ldots \ldots \ldots \ldots \ldots \ldots \ldots \ldots \ldots$

LIST OF TABLES $\ldots \ldots \ldots \ldots \ldots \ldots \ldots \ldots \ldots \ldots \ldots \ldots \ldots \ldots \ldots$

ACKNOWLEDGMENTS $\ldots \ldots \ldots \ldots \ldots \ldots \ldots \ldots \ldots \ldots \ldots \ldots \ldots \ldots \ldots$

ABSTRACT $\ldots \ldots \ldots \ldots \ldots \ldots \ldots \ldots \ldots \ldots \ldots \ldots \ldots \ldots \ldots \ldots \ldots \ldots \ldots$

PART 1: INFORMATION ABOUT THE NUMERIC DATA PACKAGE .... 1

1. NAME OF THE NUMERIC DATA PACKAGE $\ldots \ldots \ldots \ldots \ldots \ldots \ldots$

2 CONTRIBUTORS $\ldots \ldots \ldots \ldots \ldots \ldots \ldots \ldots \ldots \ldots \ldots \ldots \ldots \ldots \ldots \ldots$

3. KEYWORDS $\ldots \ldots \ldots \ldots \ldots \ldots \ldots \ldots \ldots \ldots \ldots \ldots \ldots \ldots \ldots \ldots$

4. SOURCE INFORMATION $\ldots \ldots \ldots \ldots \ldots \ldots \ldots \ldots \ldots \ldots \ldots \ldots \ldots$

5. METHODOLOGY $\ldots \ldots \ldots \ldots \ldots \ldots \ldots \ldots \ldots \ldots \ldots \ldots \ldots \ldots \ldots \ldots \ldots$

6. APPIICATIONS OF THE DATA $\ldots \ldots \ldots \ldots \ldots \ldots \ldots \ldots \ldots \ldots$

7. LIMITATIONS AND RESTRICTIONS $\ldots \ldots \ldots \ldots \ldots \ldots \ldots \ldots \ldots$

8. DATA CHECKS PERFORMED BY CDIAC $\ldots \ldots \ldots \ldots \ldots \ldots \ldots . \ldots 9$

9. REFERENCES $\ldots \ldots \ldots \ldots \ldots \ldots \ldots \ldots \ldots \ldots \ldots \ldots \ldots \ldots \ldots \ldots$

10. HOW TO OBTAIN THE PACKAGE $\ldots \ldots \ldots \ldots \ldots \ldots \ldots \ldots \ldots, 100$

PART 2: INFORMATION ABOUT THE DATA. FILES

PROVIDED ON MAGNETIC TAPE OR FLOPPY DISKETTES $\ldots \ldots \ldots \quad 103$

11. CONTENTS OF THE MAGNETIC TAPE OR FLOPPY DISKETTES $\ldots . \quad 105$

12. DESCRIPTIVE FILE ON THE TAPE/DISKETTES $\ldots \ldots \ldots \ldots \ldots \ldots$.

13. LISTLNG OF THE FORTRAN-77 DATA RETRIEVAL PROGRAM $\ldots \ldots \quad 117$

14. LISTING OF THE SAS INPUT/OUTPUT RETRIEVAL PROGRAM $\ldots$.

15. VERIFICATION OF DATA TRANSPORT $\ldots \ldots \ldots \ldots \ldots \ldots \ldots \ldots . \ldots$ 


\section{CONTENTS (continued)}

APPENDIX. REPRINTS OF PERTTNENTT LITERATURE

Determinations of carbon dioxide and methane by dual catalyst flame ionization chromatography and nitrous oxide by electron capture

chromatography, by R. F. Weiss. 1981. 


\section{LIST OF FIGURES}

Figure

Page

1 World map showing composite cruise track locations $\ldots \ldots \ldots \ldots \ldots$

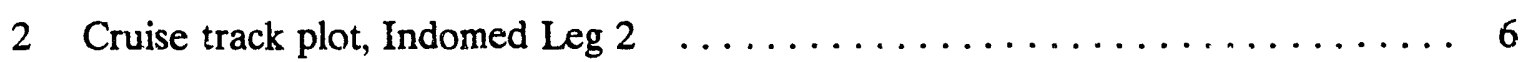

3 Data plot of $x \mathrm{~N}_{2} \mathrm{O}$ (dry gas mole fraction), Indomed Leg $2 \ldots \ldots \ldots \ldots \ldots$

4 Cruise track plot, Indomed $\operatorname{Leg} 3 \ldots \ldots \ldots \ldots \ldots \ldots \ldots \ldots \ldots \ldots \ldots \ldots \ldots$

5 Data plot of $x \mathrm{~N}_{2} \mathrm{O}$ (dry gas mole fraction), Indomed Leg $3 \ldots \ldots \ldots \ldots$

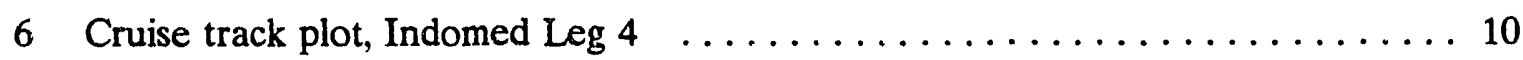

7 Data plot of $x \mathrm{~N}_{2} \mathrm{O}$ (dry gas mole fraction), Indomed Leg $4 \ldots \ldots \ldots \ldots \ldots$

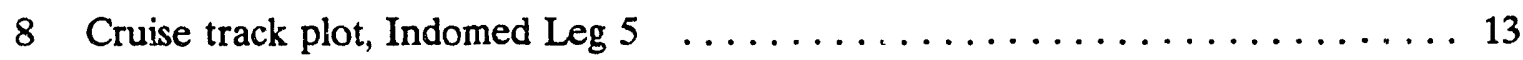

9 Data plot of $x \mathrm{~N}_{2} \mathrm{O}$ (dry gas mole fraction), Indomed Leg $5 \ldots \ldots \ldots \ldots$

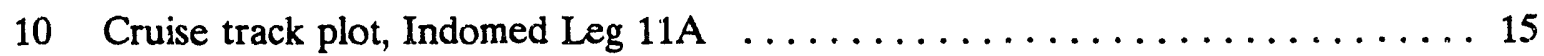

11 Data plot of $x \mathrm{CO}_{2}$ and $x \mathrm{~N}_{2} \mathrm{O}$ (dry gas mole fractions), Indomed Leg $11 \mathrm{~A} \ldots \ldots 16$

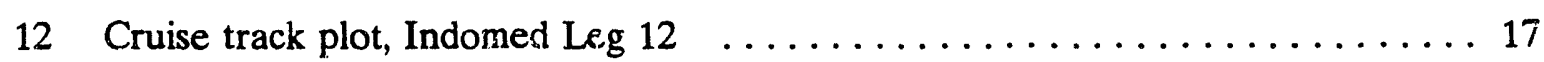

13 Data plot of $x \mathrm{CO}_{2}$ and $x \mathrm{~N}_{2} \mathrm{O}$ (dry gas mole fractions), Indomed Leg $12 \ldots \ldots 18$

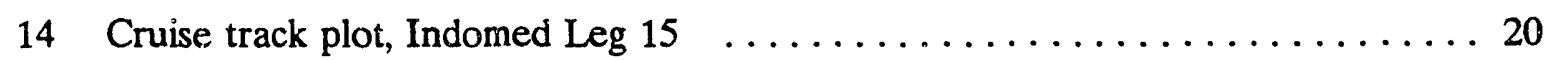

15 Data plot of $x \mathrm{CO}_{2}$ and $x \mathrm{~N}_{2} \mathrm{O}$ (dry gas mole fractions), Indomed Leg $15 \ldots \ldots 21$

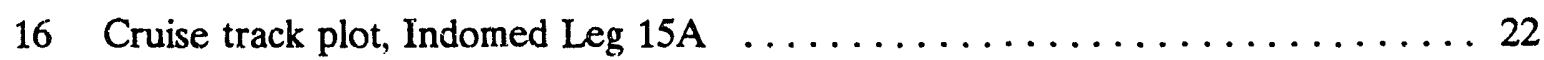

17 Data plot of $x \mathrm{CO}_{2}$ and $x \mathrm{~N}_{2} \mathrm{O}$ (dry gas mole fractions), Indomed Leg $15 \mathrm{~A} \ldots \ldots 23$

18 Cruise track plot, NORPAX Shuttle Transit $\ldots \ldots \ldots \ldots \ldots \ldots \ldots \ldots \ldots$

19 Data plot of $x \mathrm{CO}_{2}$ and $x \mathrm{~N}_{2} \mathrm{O}$ (dry gas mole fractions), NORPAX Shuttle Transit . 25

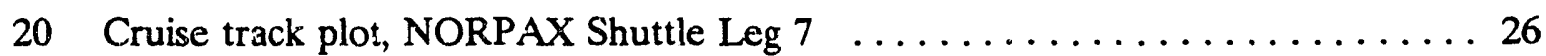

21 Data plot of $x \mathrm{CO}_{2}$ and $x \mathrm{~N}_{2} \mathrm{O}$ (dry gas mole fractions), NORPAX Shuttle Leg 7 . 27

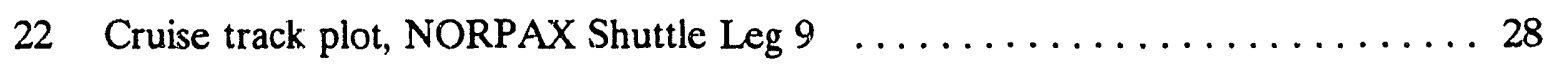




\section{LIST OF FIGURES (continued)}

Figure

$\underline{\text { Page }}$

23 Data plot of $x \mathrm{CO}_{2}$ and $x \mathrm{~N}_{2} \mathrm{O}$ (dry gas mole fractions), NORPAX Shuttle Leg $9 \ldots 29$

24 Cruise track plot, NORPAX Shuttle Leg $13 \ldots \ldots \ldots \ldots \ldots \ldots \ldots \ldots \ldots \ldots \ldots \ldots \ldots$

25 Data plot of $x \mathrm{CO}_{2}$ and $x \mathrm{~N}_{2} \mathrm{O}$ (dry gas mole fractions), NORPAX Shuttle Leg 13 . 31

26 Cruise track plot, NORPAX Shuttle Leg $15 \ldots \ldots \ldots \ldots \ldots \ldots \ldots \ldots \ldots \ldots \ldots$

27 Data plot of $x \mathrm{CO}_{2}$ and $x \mathrm{~N}_{2} \mathrm{O}$ (dry gas mole fractions), NORPAX Shuttle Leg 15 . 33

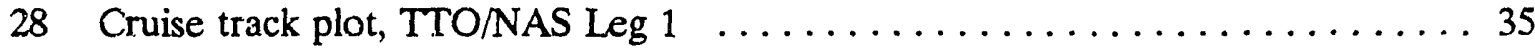

29 Data plot of $x \mathrm{CO}_{2}$ and $x \mathrm{~N}_{2} \mathrm{O}$ (dry gas mole fractions), TTO/NAS Leg $1 \ldots \ldots .36$

30 Cruise track plot, TTO/NAS Leg $2 \ldots \ldots \ldots \ldots \ldots \ldots \ldots \ldots \ldots \ldots \ldots \ldots \ldots \ldots$

31 Data plot of $x \mathrm{CO}_{2}$ and $x \mathrm{~N}_{2} \mathrm{O}$ (dry gas mole fractions), TTO/NAS Leg $2 \ldots \ldots 38$

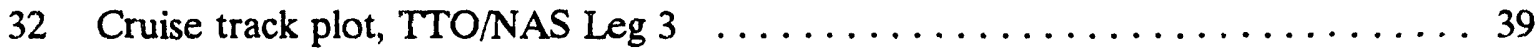

33 Data plot of $x \mathrm{CO}_{2}$ and $x \mathrm{~N}_{2} \mathrm{O}$ (dry gas mole fractions), TTO/NAS Leg $3 \ldots \ldots$

34 Cruise track plot, TTO/NAS Leg $4 \ldots \ldots \ldots \ldots \ldots \ldots \ldots \ldots \ldots \ldots \ldots \ldots$

35 Data plot of $x \mathrm{CO}_{2}$ and $x \mathrm{~N}_{2} \mathrm{O}$ (dry gas mole fractions), TTO/NAS Leg $4 \ldots \ldots 4$

36 Cruise track plot, TTO/NAS Leg $5 \ldots \ldots \ldots \ldots \ldots \ldots \ldots \ldots \ldots \ldots \ldots \ldots \ldots$

37 Data plot of $x \mathrm{CO}_{2}$ and $x \mathrm{~N}_{2} \mathrm{O}$ (dry gas mole fractions), TTO/NAS Leg $5 \ldots \ldots 4$

38 Cruise track plot, TTO/NAS Leg $6 \ldots \ldots \ldots \ldots \ldots \ldots \ldots \ldots \ldots \ldots \ldots \ldots \ldots$

39 Daca plot of $x \mathrm{CO}_{2}$ and $x \mathrm{~N}_{2} \mathrm{O}$ (dry gas mole fractions), TTO/NAS Leg $6 \ldots 46$

40 Cruise track plot, TTO/NAS Leg $7 \ldots \ldots \ldots \ldots \ldots \ldots \ldots \ldots \ldots \ldots \ldots \ldots$

41 Data plot of $x \mathrm{CO}_{2}$ and $x \mathrm{~N}_{2} \mathrm{O}$ (dry gas mole fractions), TTO/NAS Leg $7 \ldots \ldots 8$

42 Cruise track plot, Hudson $82-001$ Leg $1 \ldots \ldots \ldots \ldots \ldots \ldots$

43 Data plot of $x \mathrm{CO}_{2}$ and $x \mathrm{~N}_{2} \mathrm{O}$ (dry gas mole fractions), Hudson 82-001 Leg $1 \ldots 50$

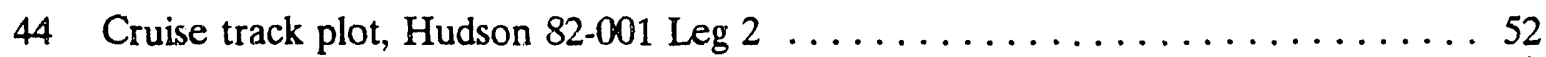




\section{LIST OF FIGURES (continued)}

Figure

Page

45 Data plot of $x \mathrm{CO}_{2}$ and $x \mathrm{~N}_{2} \mathrm{O}$ (dry gas mole fractions), Hudson 82-001 Leg $2 \ldots 53$

46 Cruise track plot, TTO/TAS Leg $1 \ldots \ldots \ldots \ldots \ldots \ldots \ldots \ldots$

47 Data plot of $x \mathrm{CO}_{2}$ and $x \mathrm{~N}_{2} \mathrm{O}$ (dry gas mole fractions), TTO/TAS Leg $1 \ldots 55$

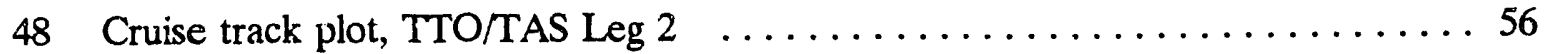

49 Data plot of $x \mathrm{CO}_{2}$ and $x \mathrm{~N}_{2} \mathrm{O}$ (dry gas mole fractions), TTO/TAS Leg $2 \ldots 57$

50 Cruise track plot, TTO/TAS Leg $3 \ldots \ldots \ldots \ldots \ldots \ldots \ldots$

51 Data plot of $x \mathrm{CO}_{2}$ and $x \mathrm{~N}_{2} \mathrm{O}$ (dry gas mole fractions), TTO/TAS Leg $3 \ldots 59$

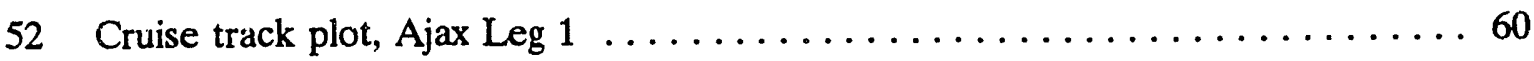

53 Data plot of $x \mathrm{CO}_{2}$ and $x \mathrm{~N}_{2} \mathrm{O}$ (dry gas mole fractions), Ajax Leg $1 \ldots \ldots 1$

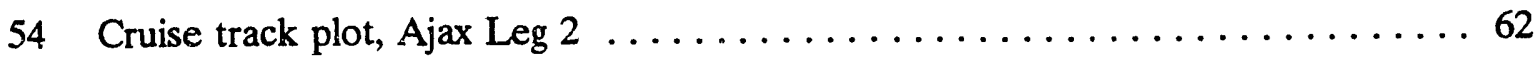

55 Data plot of $x \mathrm{CO}_{2}$ and $x \mathrm{~N}_{2} \mathrm{O}$ (dry gas mole fractions), Ajax Leg $2 \ldots \ldots 3$

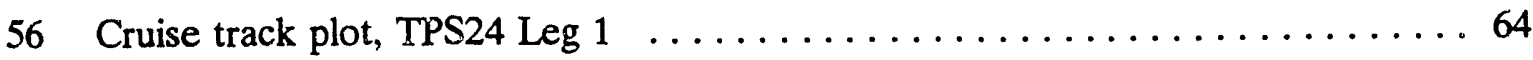

57 Data plot of $x \mathrm{CO}_{2}$ and $x \mathrm{~N}_{2} \mathrm{O}$ (dry gas mole fractions), TPS24 Leg $1 \ldots \ldots 5$

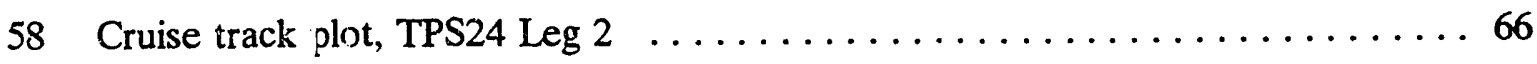

59 Data plot of $x \mathrm{CO}_{2}$ and $x \mathrm{~N}_{2} \mathrm{O}$ (dry gas mole fractions), TPS24 Leg $2 \ldots 67$

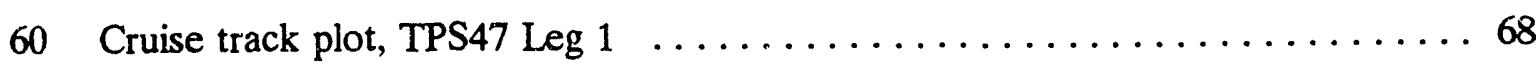

61 Data plot of $x \mathrm{CO}_{2}$ and $x \mathrm{~N}_{2} \mathrm{O}$ (dry gas mole fractions), TPS47 L:g $1 \ldots 69$

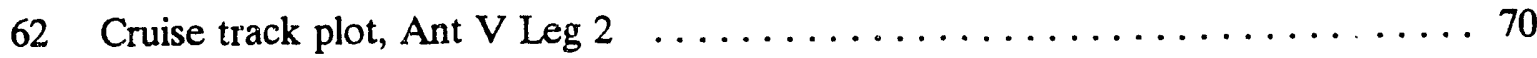

63 Data plot of $x \mathrm{CO}_{2}$ and $x \mathrm{~N}_{2} \mathrm{O}$ (dry gas mole fractions), Ant $\mathrm{V} \mathrm{Leg} 2 \ldots \ldots 71$

64 Cruise track plot, Ant V Leg $3 \ldots \ldots \ldots \ldots \ldots \ldots \ldots \ldots \ldots \ldots$

65 Data plot of $x \mathrm{CO}_{2}$ and $x \mathrm{~N}_{2} \mathrm{O}$ (dry gas mole fractions), Ant $\mathrm{V} \mathrm{Leg} 3 \ldots 75$

66 Cruise track plot, SAVE Transit $\ldots \ldots \ldots \ldots \ldots \ldots \ldots \ldots \ldots \ldots \ldots$ 


\section{LIST OF FIGURES (continued)}

$\underline{\text { Figure }}$

67 Data plot of $x \mathrm{CO}_{2}$ and $x \mathrm{~N}_{2} \mathrm{O}$ (dry gas mole fractions), SAVE Transit $\ldots \ldots \ldots 78$

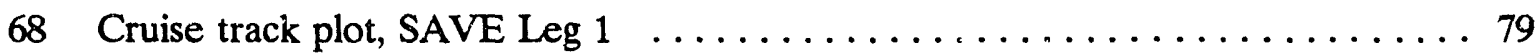

69 Data plot of $x \mathrm{CO}_{2}$ and $x \mathrm{~N}_{2} \mathrm{O}$ (dry gas mole fractions), SAVE Leg $1 \ldots \ldots 0$

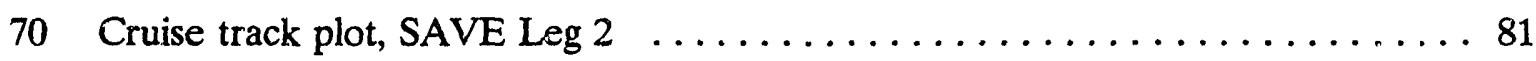

71 Data plot of $x \mathrm{CO}_{2}$ and $x \mathrm{~N}_{2} \mathrm{O}$ (dry gas mole fractions), SAVE Leg $2 \ldots \ldots 2$

72 Cruise track plot, SAVE Leg $3 \ldots \ldots \ldots \ldots \ldots \ldots \ldots \ldots \ldots \ldots \ldots \ldots$

73 Data plot of $x \mathrm{CO}_{2}$ and $x \mathrm{~N}_{2} \mathrm{O}$ (dry gas mole fractions), SAVE Leg $3 \ldots \ldots 4$

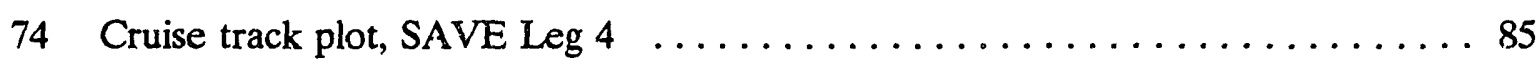

75 Data plot of $x \mathrm{CO}_{2}$ and $x \mathrm{~N}_{2} \mathrm{O}$ (dry gas mole fractions), SAVE Leg $4 \ldots \ldots 6$

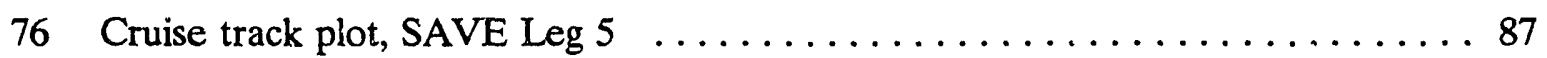

77 Data plot of $x \mathrm{CO}_{2}$ and $x \mathrm{~N}_{2} \mathrm{O}$ (dry gas mole factions), SAVE Leg $5 \ldots \ldots 8$

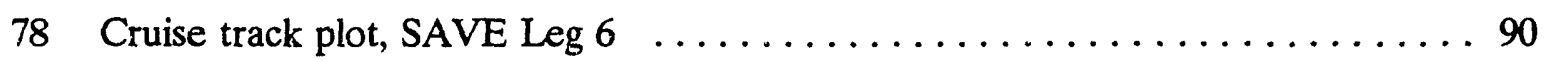

79 Data plot of $x \mathrm{CO}_{2}$ and $x \mathrm{~N}_{2} \mathrm{O}$ (dry gas mole fractions), SAVE Leg $6 \ldots \ldots$. . 91

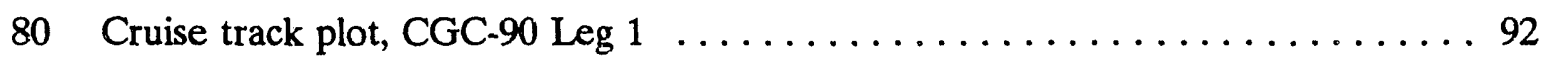

81 Data plot of $x \mathrm{CO}_{2}$ and $x \mathrm{~N}_{2} \mathrm{O}$ (dry gas mole fractions), CuC-90 Leg $1 \ldots 93$

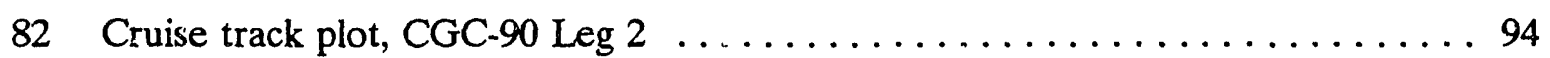

83 uata plot of $x \mathrm{CO}_{2}$ and $x \mathrm{~N}_{2} \mathrm{O}$ (dry gas mole fractions), CGC-90 Leg $2 \ldots 95$ 


\section{LIST OF TABLES}

Table

Page

1 Track list of expeditions that contributed measurements to the surface water and atmospheric $\mathrm{CO}_{2}$ and $\mathrm{N}_{2} \mathrm{O}$ data set $\ldots \ldots \ldots \ldots \ldots 4$

2 Partial listing of one of the surface water and atmospheric $\mathrm{CO}_{2}$ and $\mathrm{N}_{2} \mathrm{O}$ data files (File 23 on the magnetic tape or NORPAX0.H2:O on the floppy diskette)

3 Characteristics of numeric variables in the collective surface water and atmospheric $\mathrm{CO}_{2}$ and $\mathrm{N}_{2} \mathrm{O}$ data files 120 


\section{ACKNOWLEDGMENTS}

This report contains the results of many years of work which could not have been completed without the help of many colleagues. We are especially graieful to J. Bullister, E. Dlugokencky, K. Harrison, E. Hoopes, P. Murphy, M. Warner, and the officers and crews of the many research vessels upon which we have worked for their assistance with the shipboard operations. This work was supported by grants from the Marine Chemistry and Physical Oceanography programs of the National Science Foundation and from the Department of Energy, often as an ancillary part of our seagoing chlorofluorocarbon tracer measurement efforts. 


\begin{abstract}
R. F. WEISS, F. A. VAN WOY, AND P. K. SALAMEH. 1992. Surface water and atmospheric carbon dioxide and nitrous oxide observations by shipboard automated gas chromatography: Results from expeditions between 1977 and 1990. Scripps Institution of Oceanography Reference 92-11. ORNL/CDIAC-59, NDP-044. Carbon Dioxide Information Analysis Center, Oak Ridge National Laboratory, Oak Ridge, Tennessee. 144 pp.
\end{abstract}

This document presents the results of surface water and atmospheric carbon dioxide $\left(\mathrm{CO}_{2}\right)$ and nitrous oxide $\left(\mathrm{N}_{2} \mathrm{O}\right)$ measurements carried out by shipboard gas chromatography over the period 1977-1990. These data include results from 11 different oceanic surveys for a total of 41 expedition legs. Collectively, they represent a globally distributed sampling that includes locations in the Atlantic, Pacific, Indian, and Southern Oceans, as well as ihe Mediterranean and Red Seas.

The measurements were made by an automated high-precision shipboard gas chromatographic system developed during the late 1970s and used extensively over the intervening years. This instrument measures $\mathrm{CO}_{2}$ by flame ionization after quantitative reaction to methane in a stream of hydrogen. Nitrous oxide is measured by a separate electron capture detector. The chromatographic system measures 196 dry-gas samples a day, divided equally among the atmosphere, gas equilibrated with surface water, a low-range gas standard, and a high-range gas standard.

These data constitute one of the most extensive records available of $\mathrm{CO}_{2}$ and, particularly, $\mathrm{N}_{2} \mathrm{O}$ in marine air and surface seawater. The data will be valuable in modeling applications dealing with the ocean's role in the global cycles of carbon and nitrogen, in studies of ocean-atmosphere dynamics, and in studies evaluating other methodologies for determining $p \mathrm{CO}_{2}$.

All data have been assessed for quality and consistency and have been edited to remove serious outliers and contaminated samples and to correct gross numerical errors.

These data are arailable free of charge as a numeric data package (NDP) from the Carbon Dioxide Information Analysis Center (CDIAC). The NDP consists of this document and a magnetic tape (or set of floppy diskettes) containing machine-readable files. ${ }^{a}$ This document provides sample listings of the surface water and atmospheric $\mathrm{CO}_{2}$ and $\mathrm{N}_{2} \mathrm{O}$ data, offers retrieval program listings (in FORTRAN and SAS ${ }^{b}$ languages), furnishes graphical and tabular information on each of the contributing oceanic expeditions, defines limitations and restrictions of the data, and reprints pertinent literature.

\footnotetext{
${ }^{a}$ Files are also available through Internet using the File Transfer Protocol (FTP) from CDIAC's anonymous FTP area.

${ }^{b}$ SAS is the registered trademark of SAS Institute, Inc., Cary, NC $27511-8000$.
} 
PART 1

INFORMATION ABOUT THE NUMERIC DATA PACKAGE 


\section{NAME OF THE NUMERIC DATA PACKAGE}

Surface Water and Atmospheric Carbon Dioxide and Nitrous Oxide Observations by Shipboard Automated Gas Chromatography: Results from Expeditions between 1977 and 1990

\section{CONTRIBUTORS}
R. F. Weiss
F. A. Van Woy
P. K. Salameh

Scripps Institution of Oceanography

University of California, San Diego

La Jolla, California

\section{KEYWORDS}

Carbon dioxide $\left(\mathrm{CO}_{2}\right)$; gas chromatography; marine atmospheric concentrations; nitrous oxide $\left(\mathrm{N}_{2} \mathrm{O}\right)$; oceanography; surface seawaier dissolved gases.

\section{SOURCE INFORMATION}

The surface water and atmospheric carbon dioxide $\left(\mathrm{CO}_{2}\right)$ and nitrous oxide $\left(\mathrm{N}_{2} \mathrm{O}\right)$ data reported here were obtained by direct shipboard gas chromatographic measurement. These data include results from 11 different oceanic surveys for a total of 41 expedition legs. The represented oceanic surveys include the following: (1) the Indomed expedition, 1977-1979 [Indomed legs 4 and 5 are also part of the Geochemical Ocean Sections (GEOSECS) Indian Ocean expedition]; (2) the North Pacific Experiment (NORPAX) Hawaii-Tahiti Shuttle Experiment, 1979-1980; (3) and (4) the Transient Tracers in the Ocean, North Atlantic and Tropical Atlantic Studies (TTO/NAS, TTO/TAS), 1981-1983; (5) the Hudson 82-001 expedition, 1982; (6) the Ajax expedition, 1983-1984; (7) and (8) the Trans-Pacific Sections expeditions along 24 degrees North and 47 degrees North (TPS24 and TPS47), 1985; (9) the fifth "Antarktis" expedition (Ant V) of the R/V Polarstern, as part of the Winter Weddell Sea Experiment, 1986; (10) the South Atlantic Ventilation Experiment (SAVE), 1987-1989 (SAVE legs 4-6 are also designated as legs 2-4 of R/V Melville's Hydros expedition); and (11) the 1990 expedition of the National Oceanic and Atmospheric Administration's Climate and Global Change series (CGC-90). Table 1 presents a track list showing the dates, ports of departure and arrival, regions surveyed, and cruise ship names for each of the 41 expedition legs that contributed data. In addition, a series of maps showing the tracks of the expeditions and the $\mathrm{N}_{2} \mathrm{O}$ and $\mathrm{CO}_{2}$ results for each expedition leg is presented in Figs. 1-83. 
Table 1. Track list of expeditions that contributed measurements to the surface water and atmospheric $\mathrm{CO}_{2}$ and $\mathrm{N}_{2} \mathrm{O}$ data set

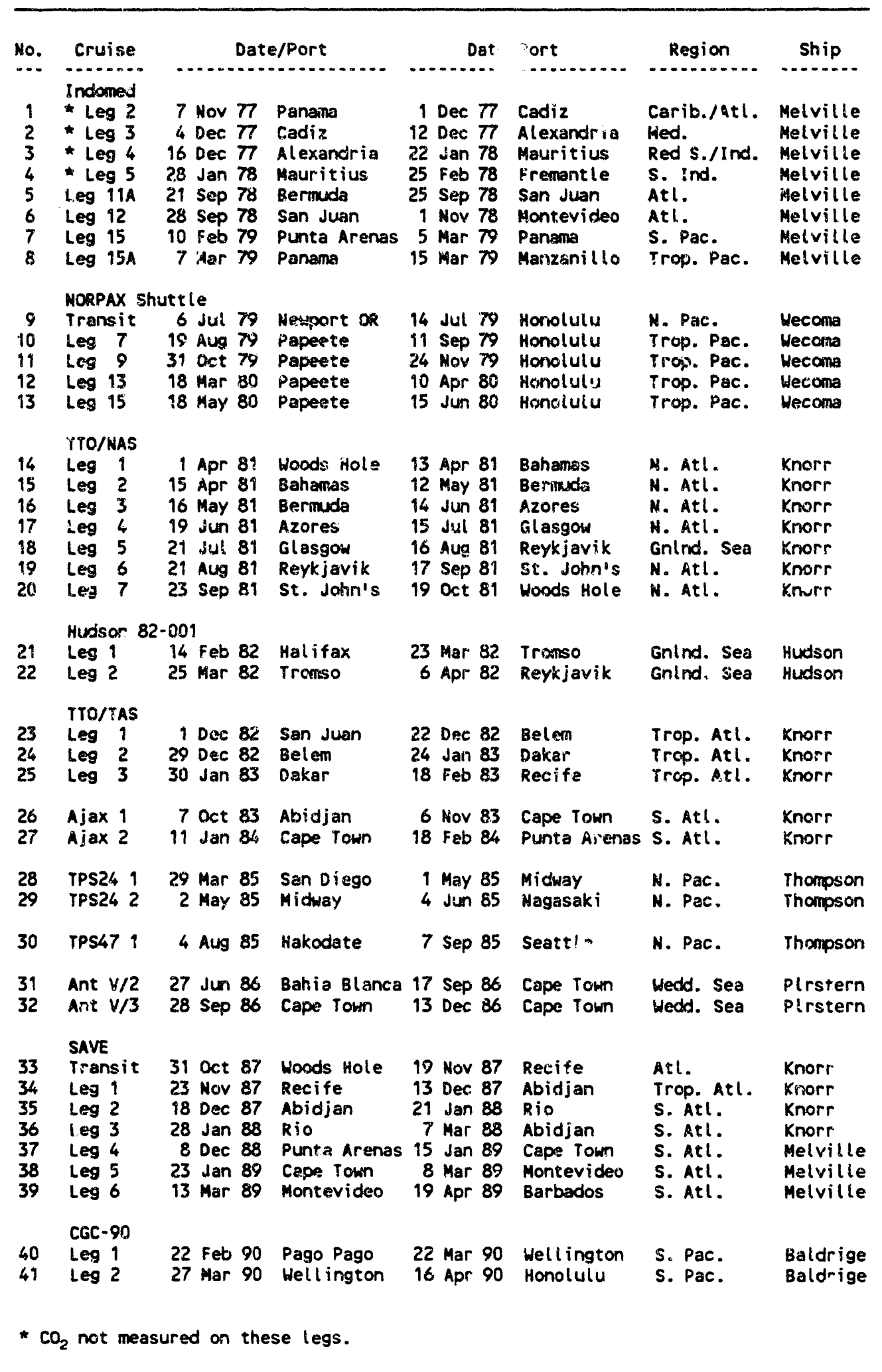




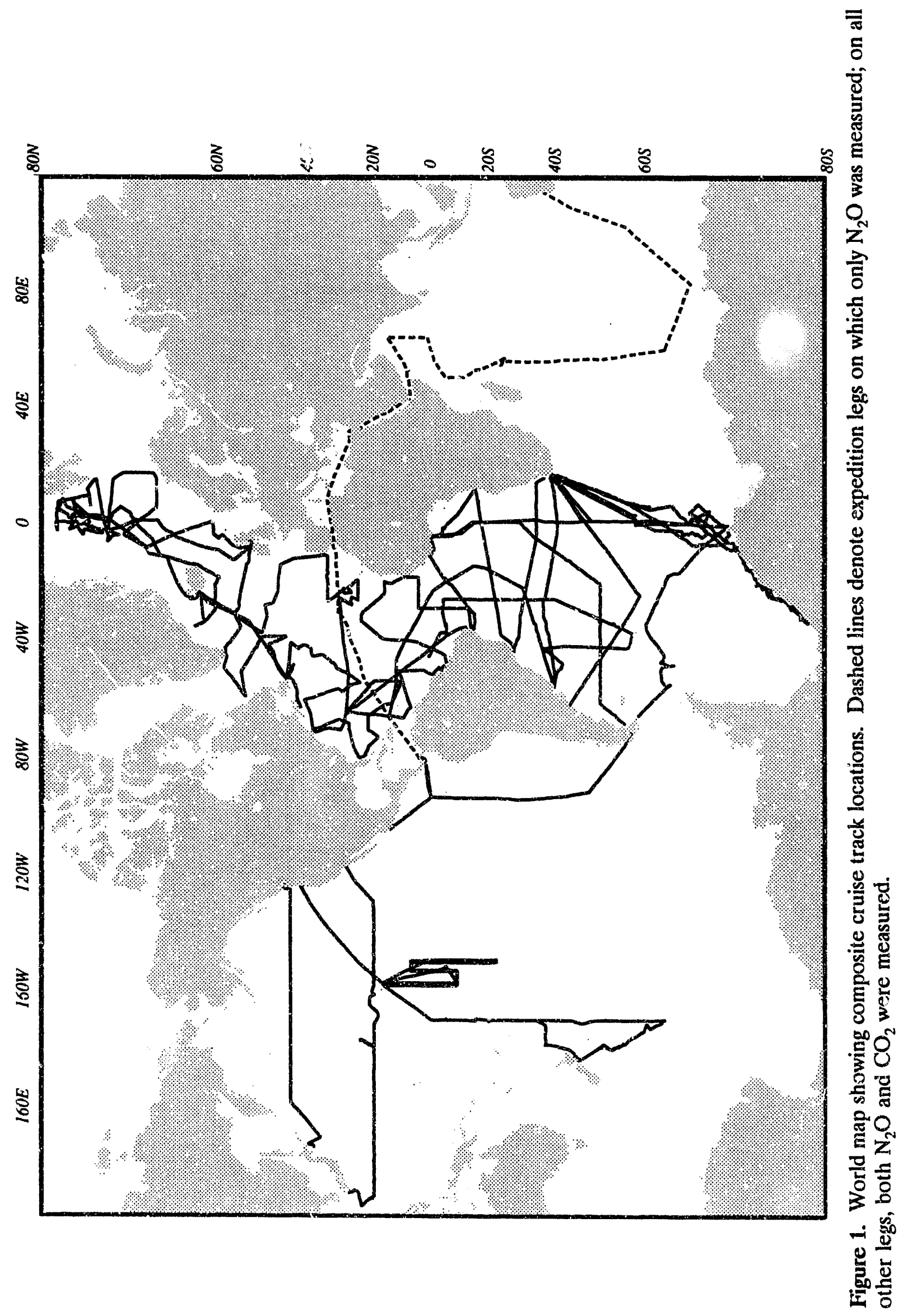




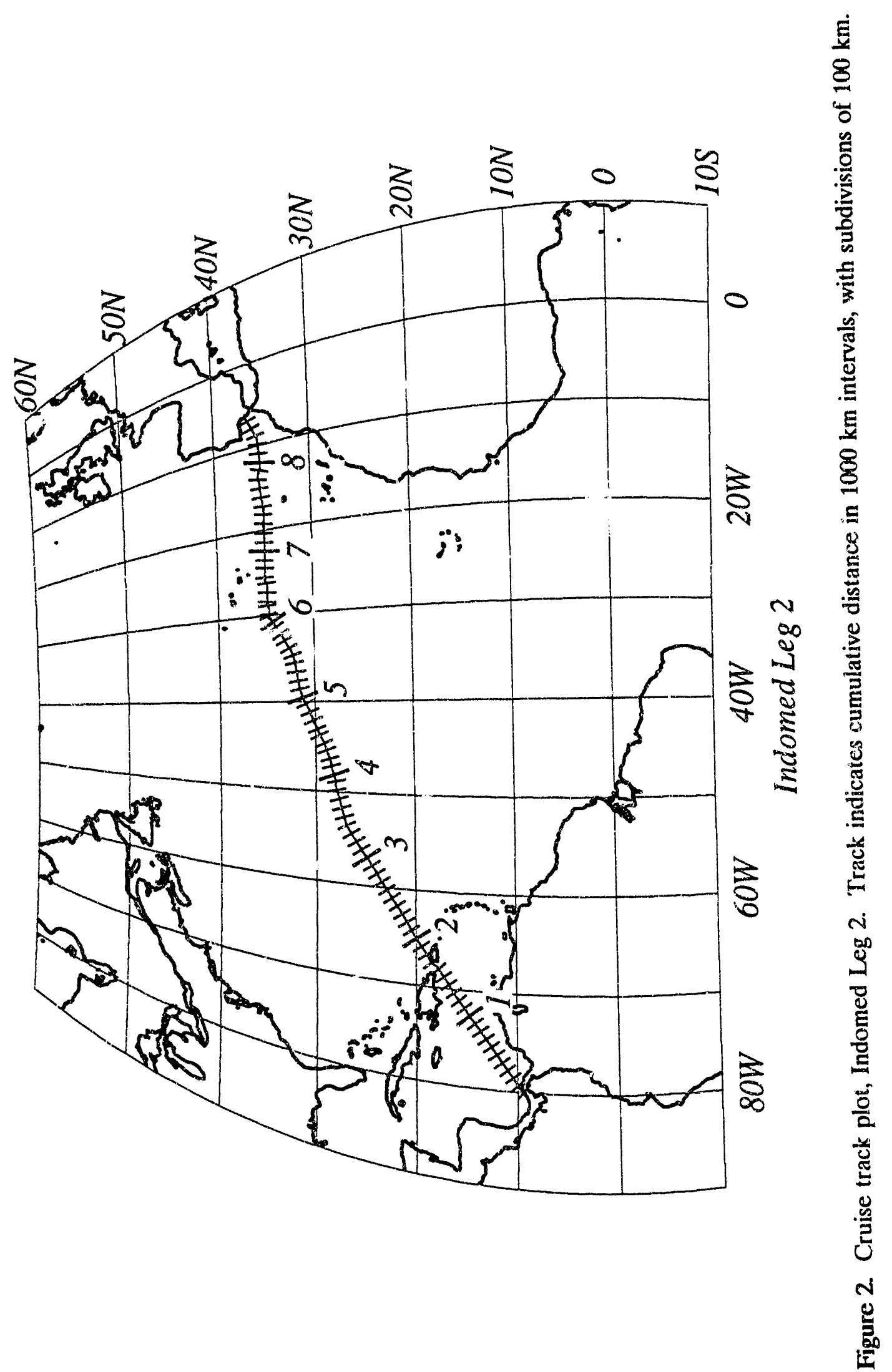




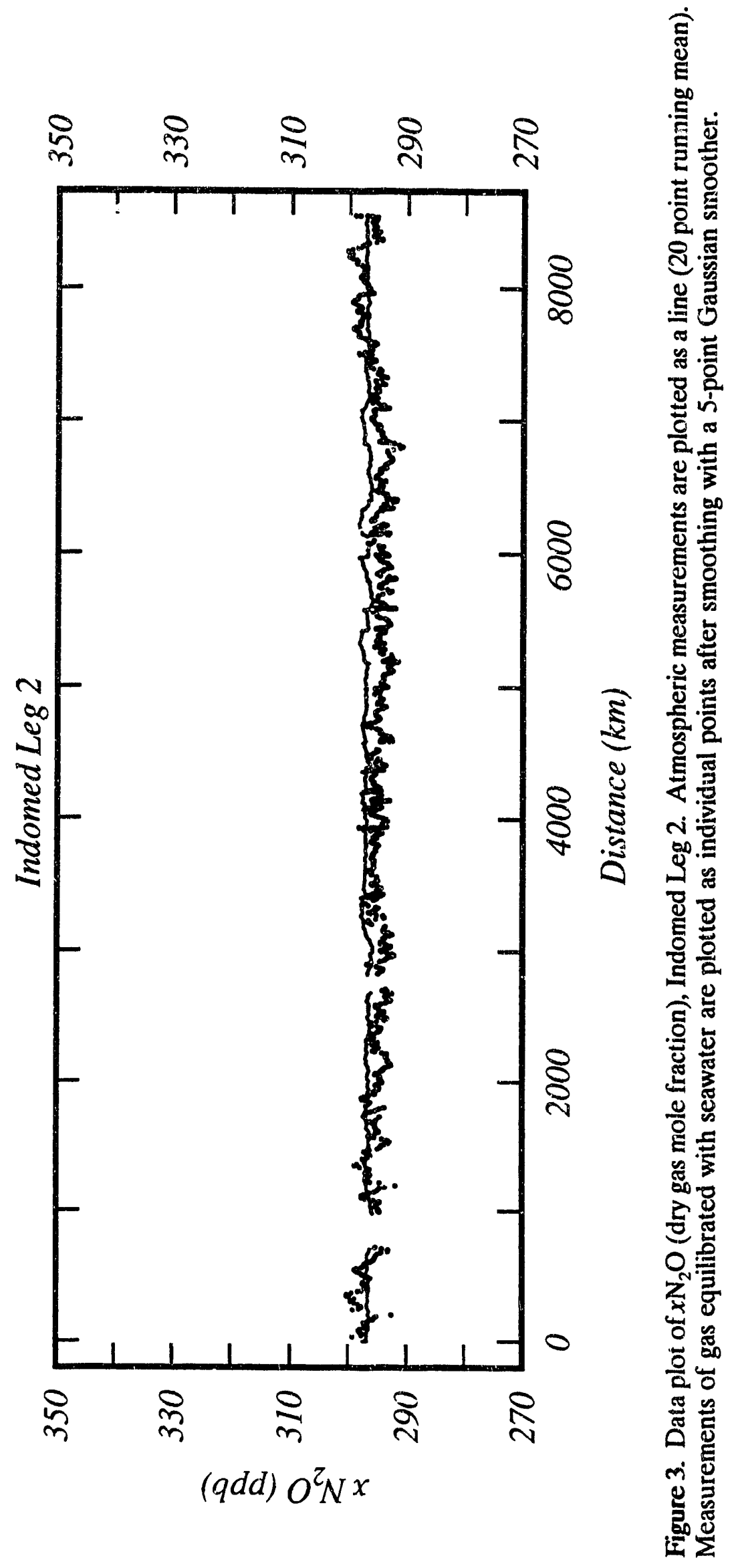




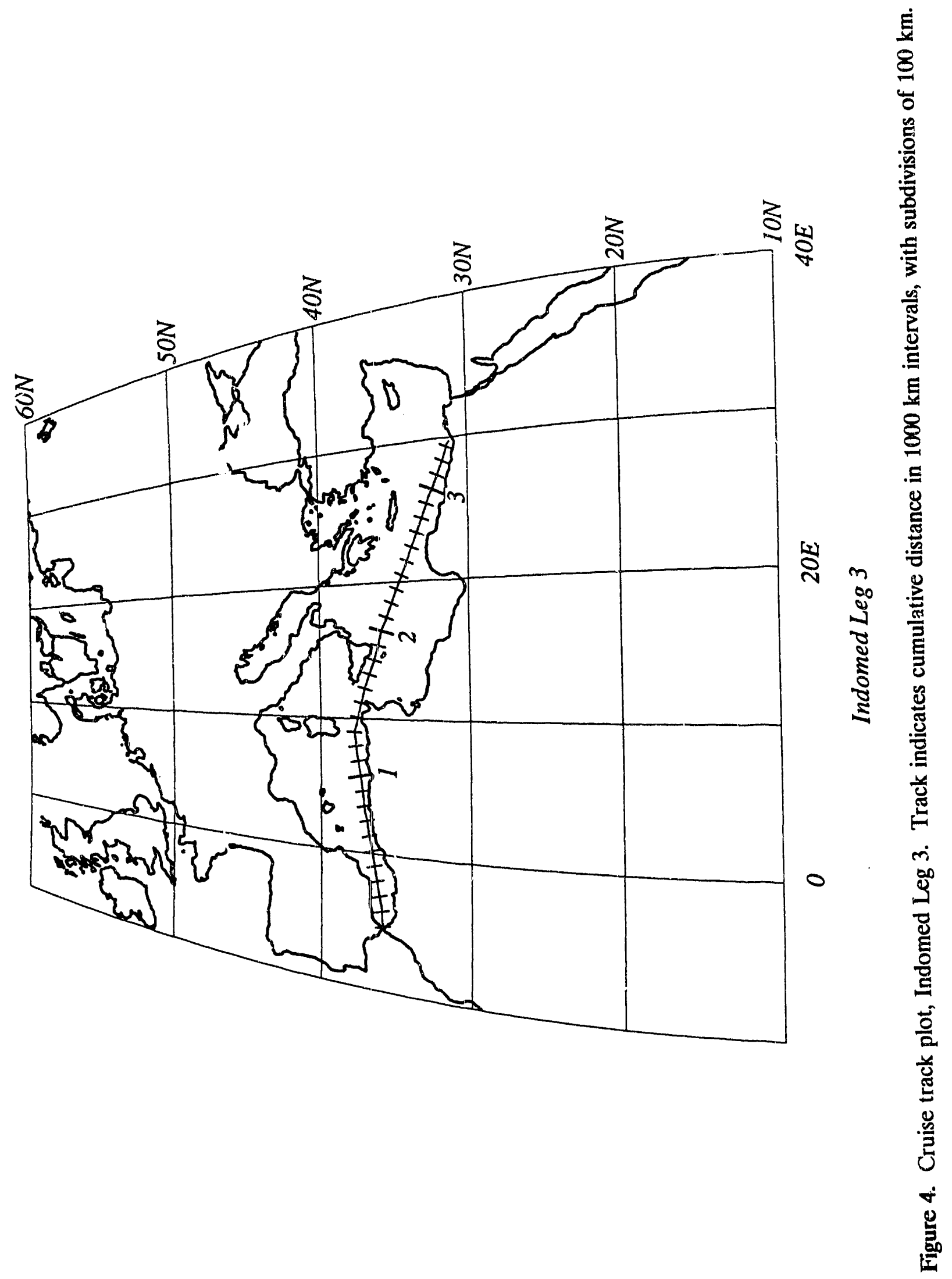



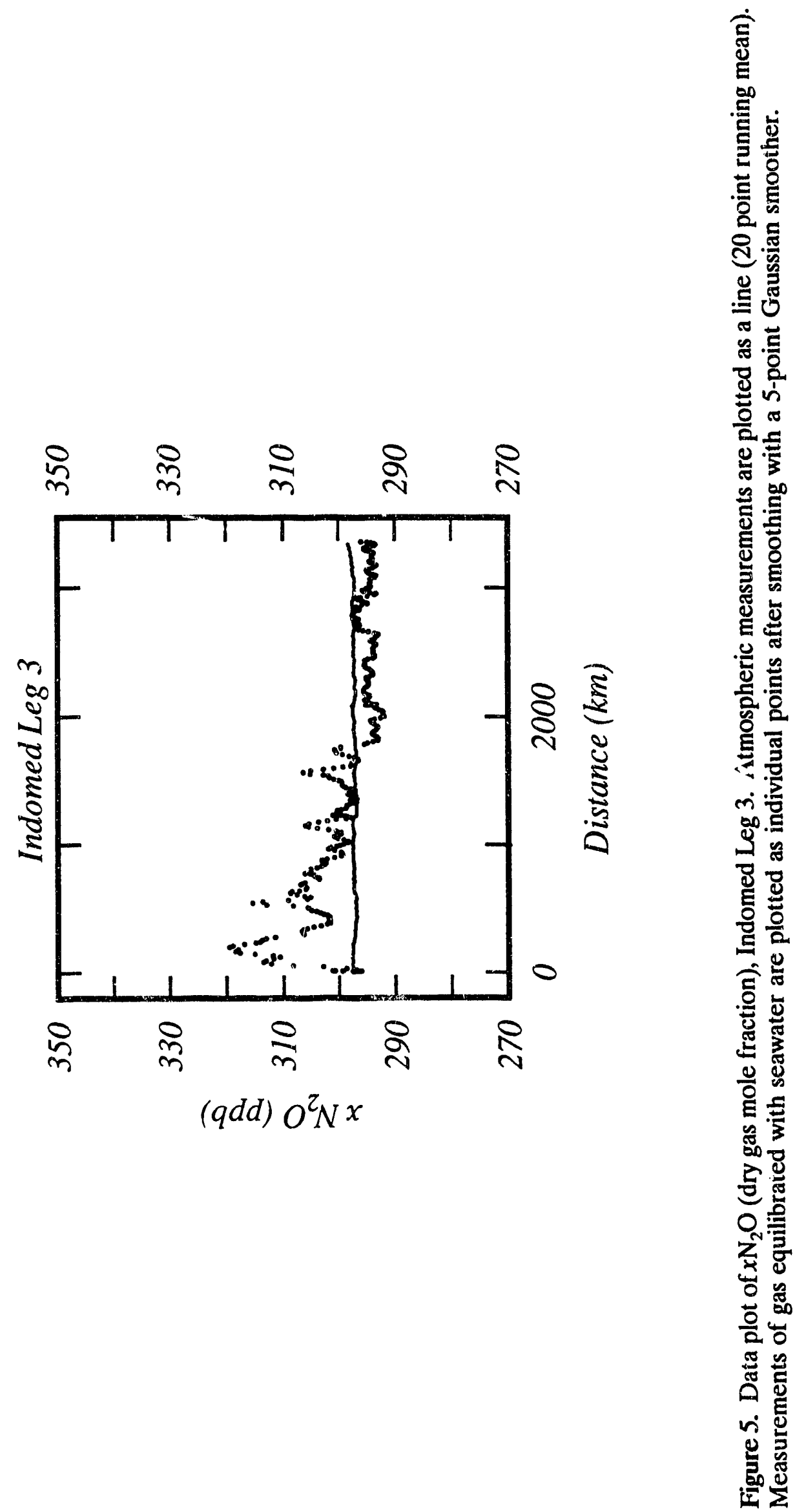


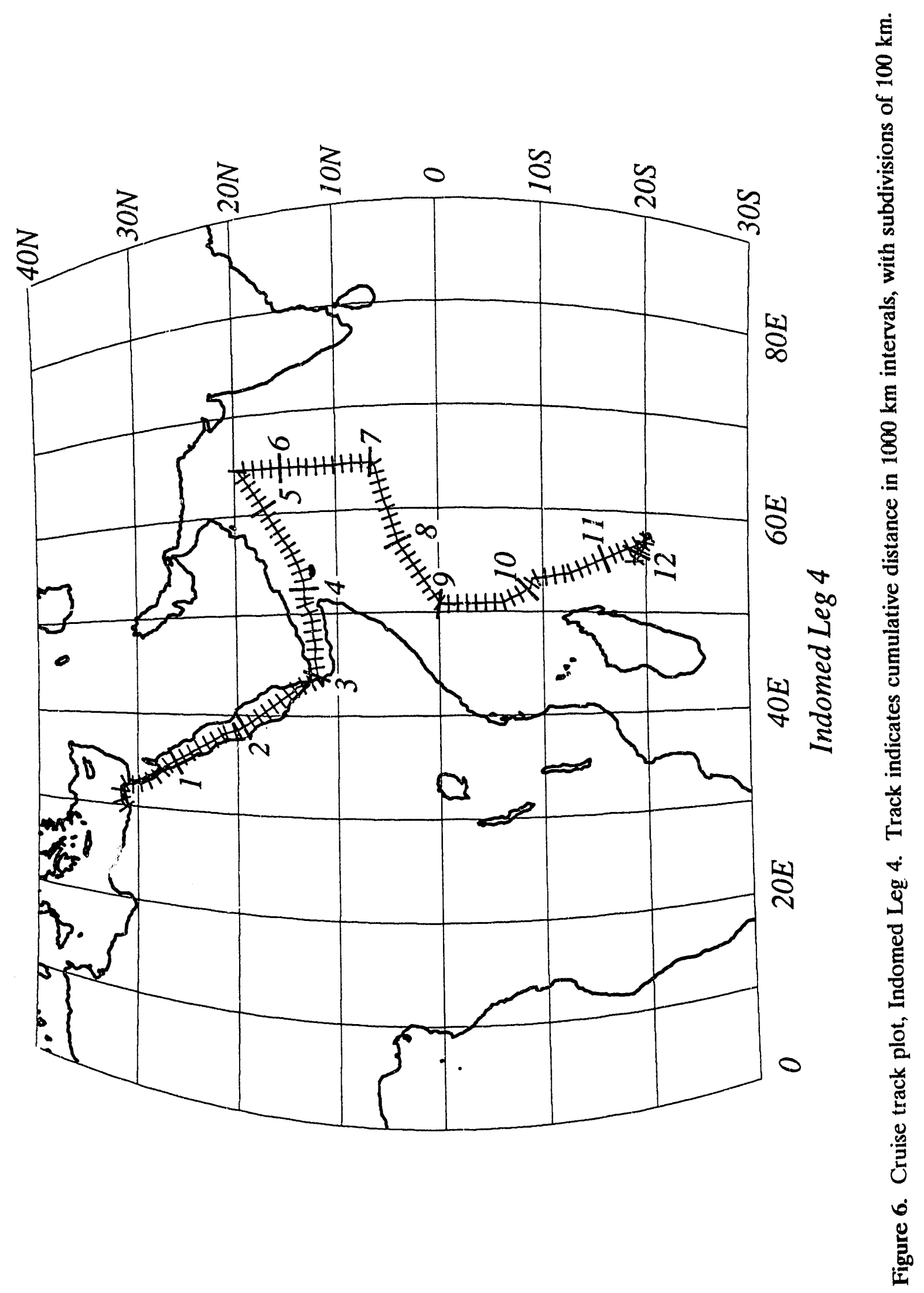




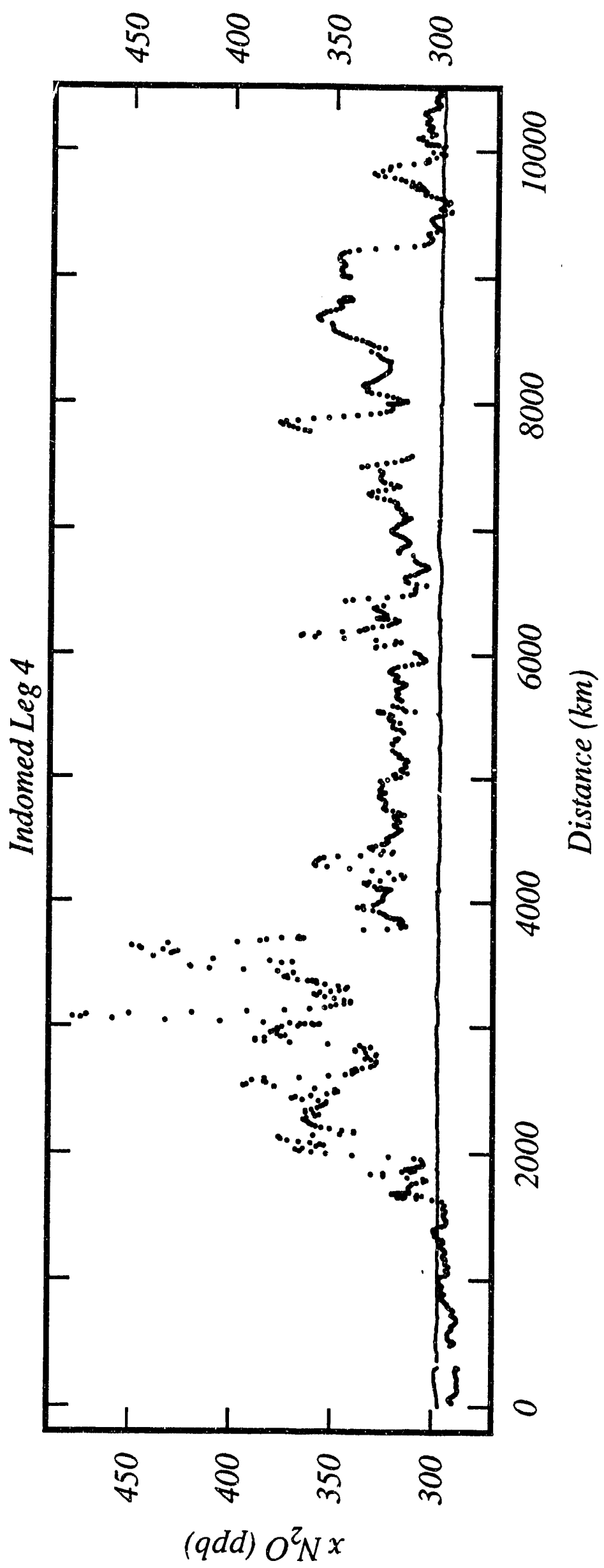

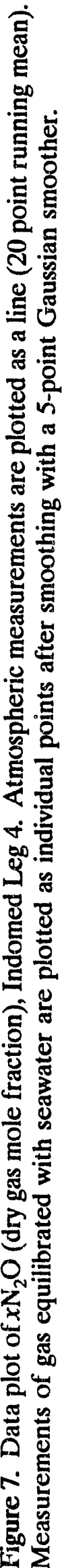




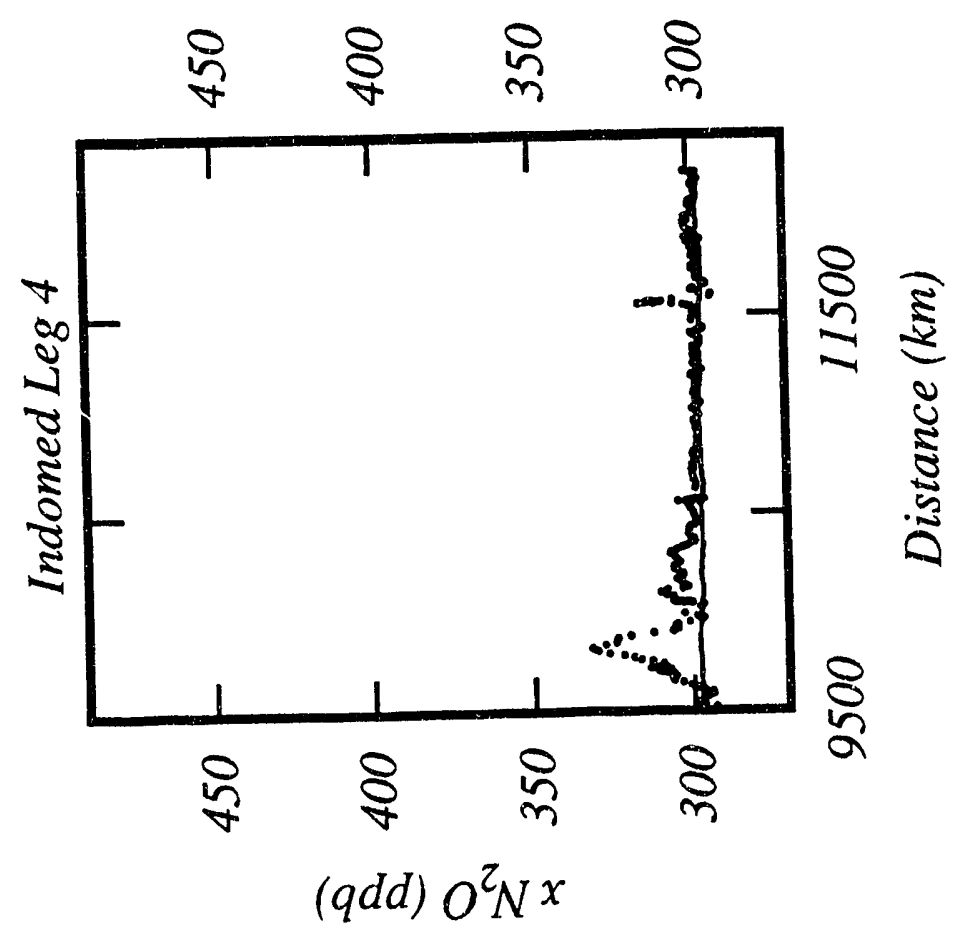

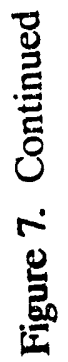




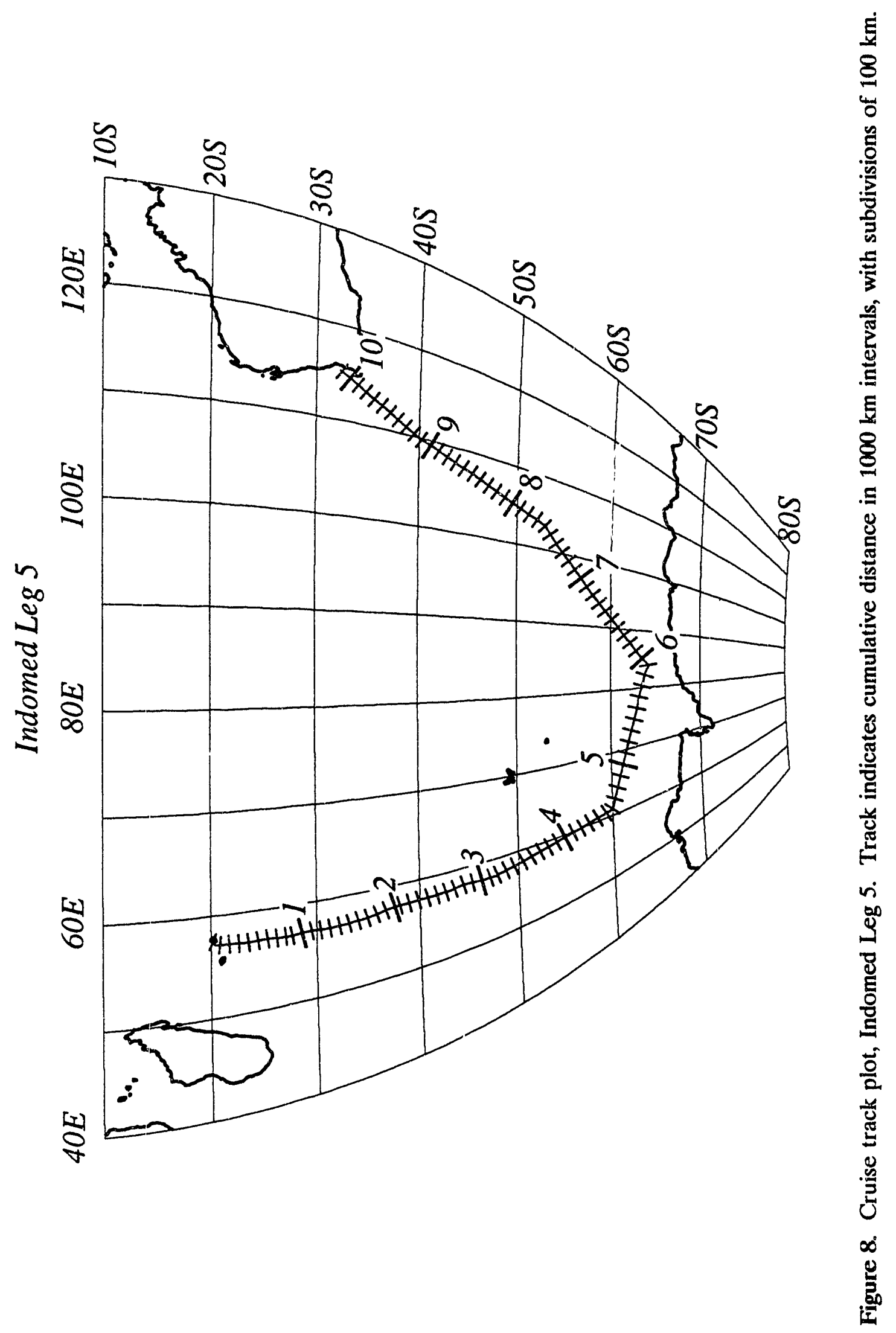




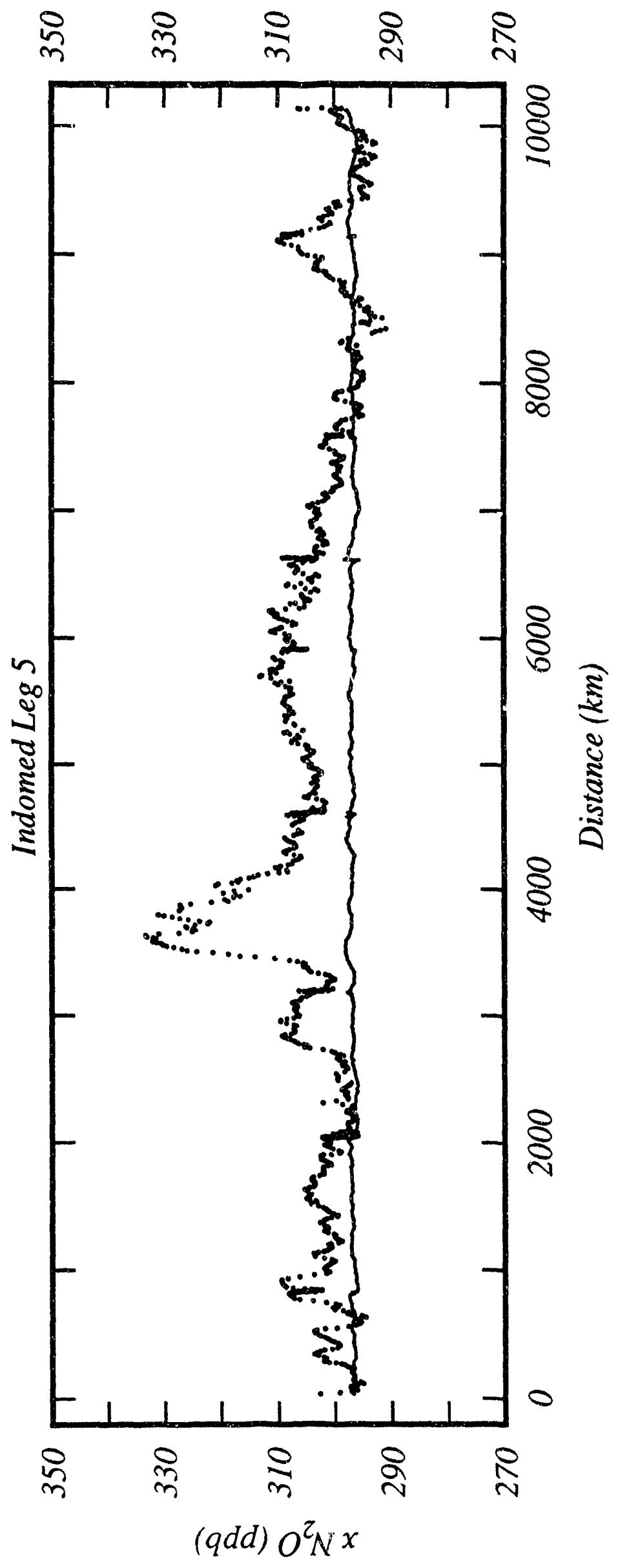

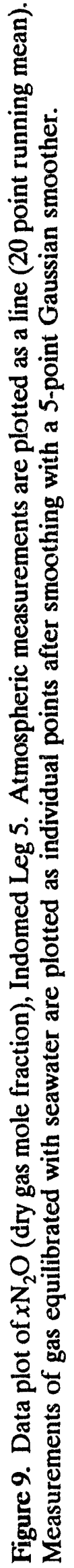




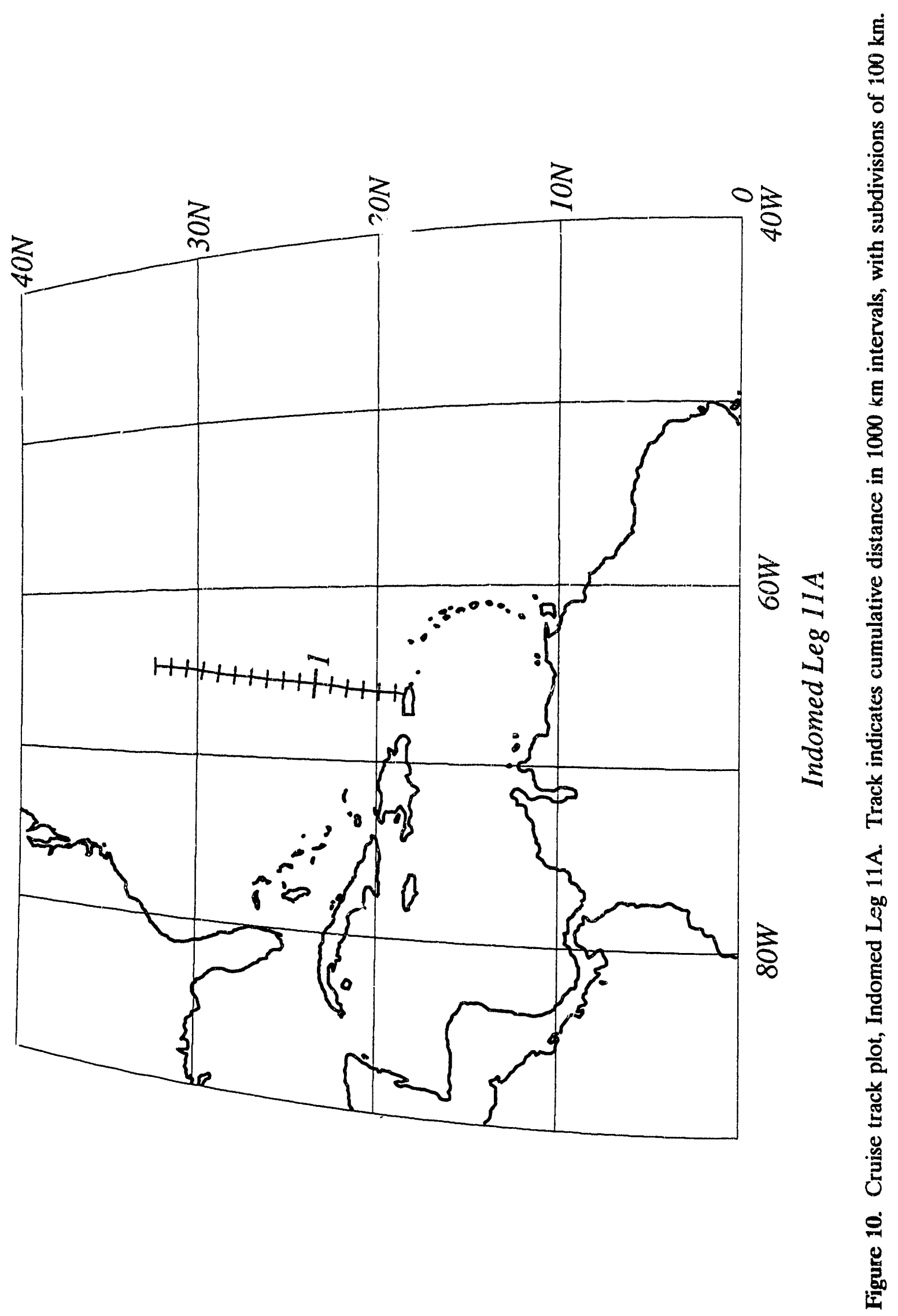




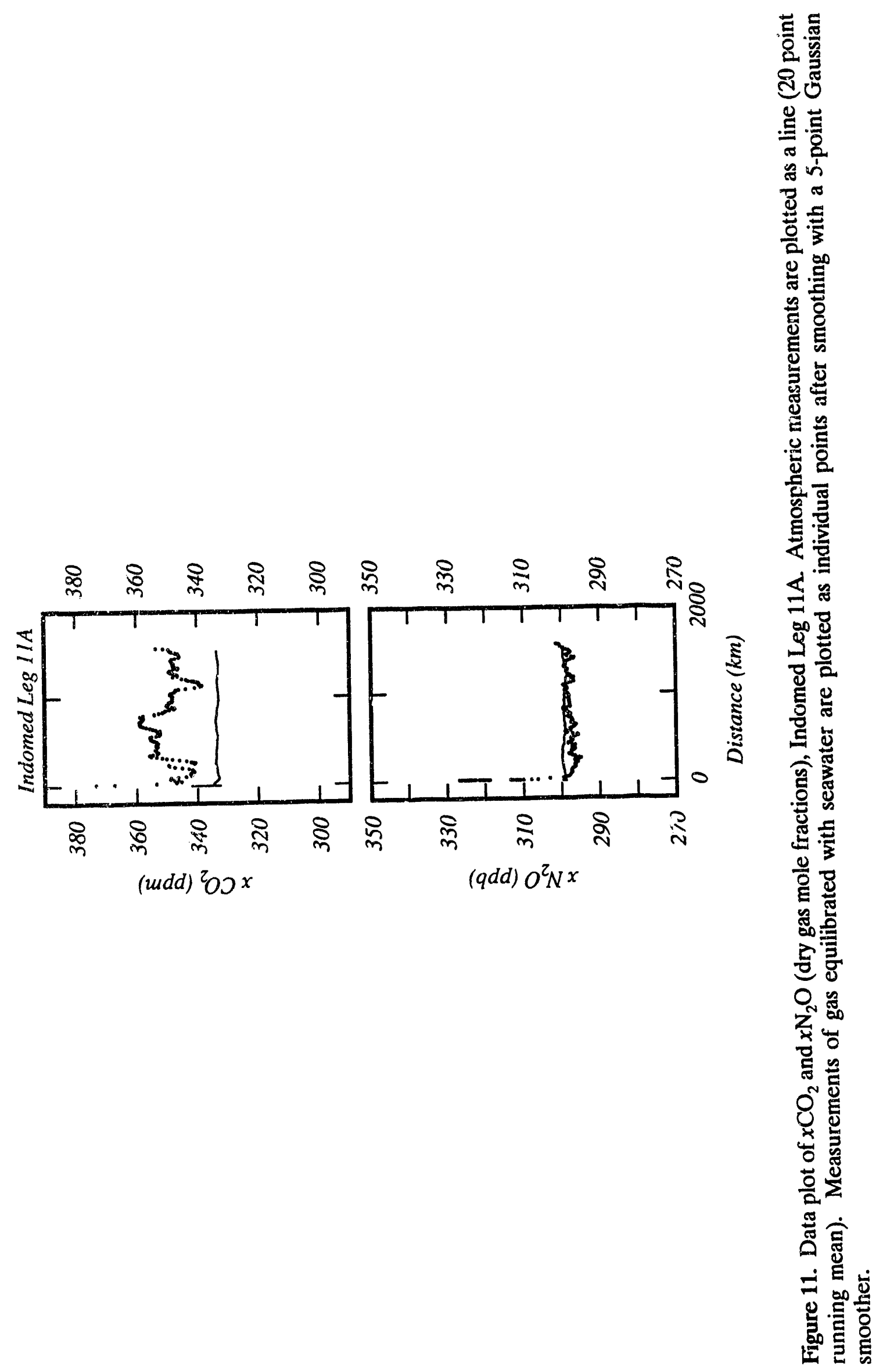




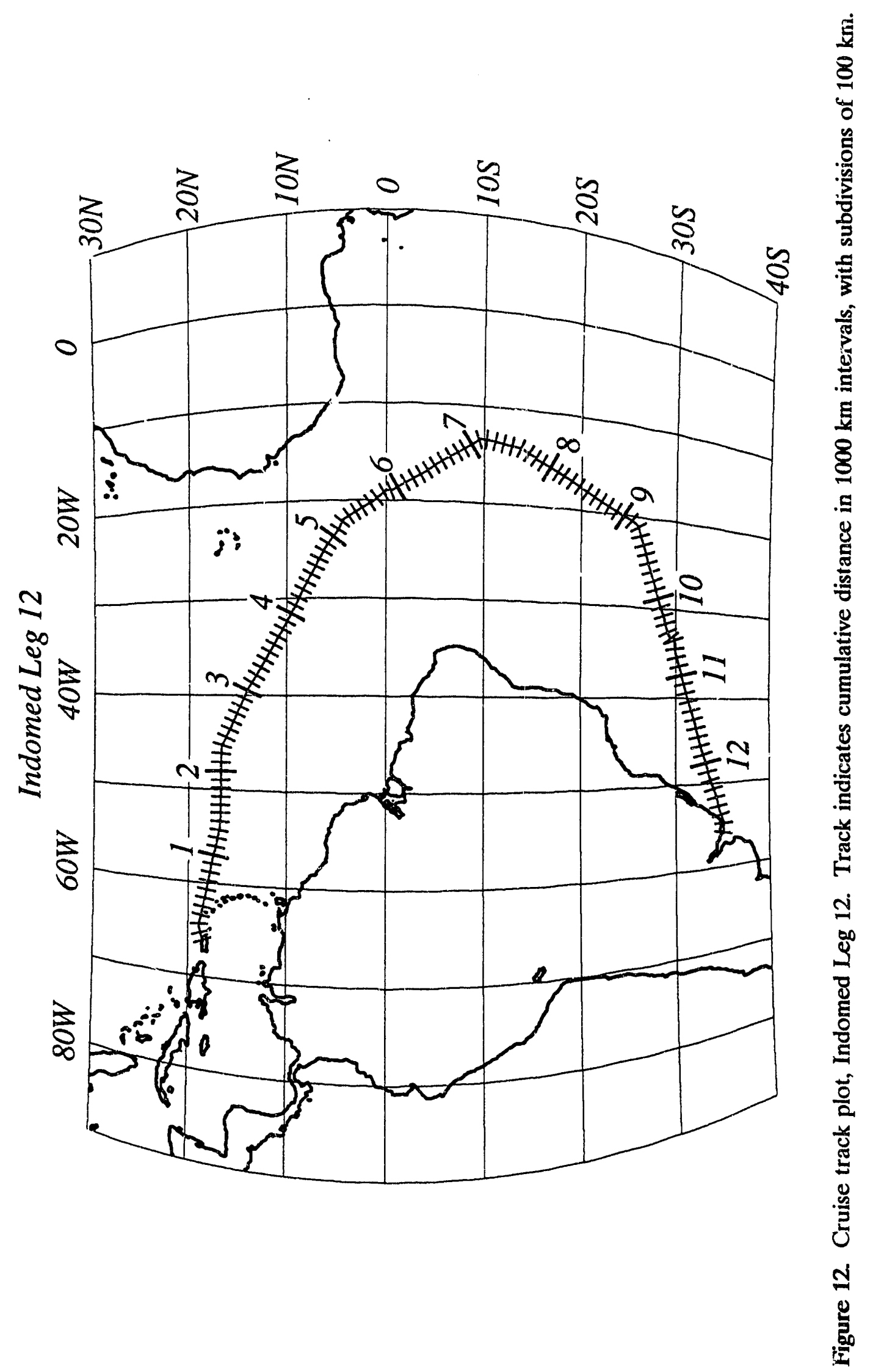




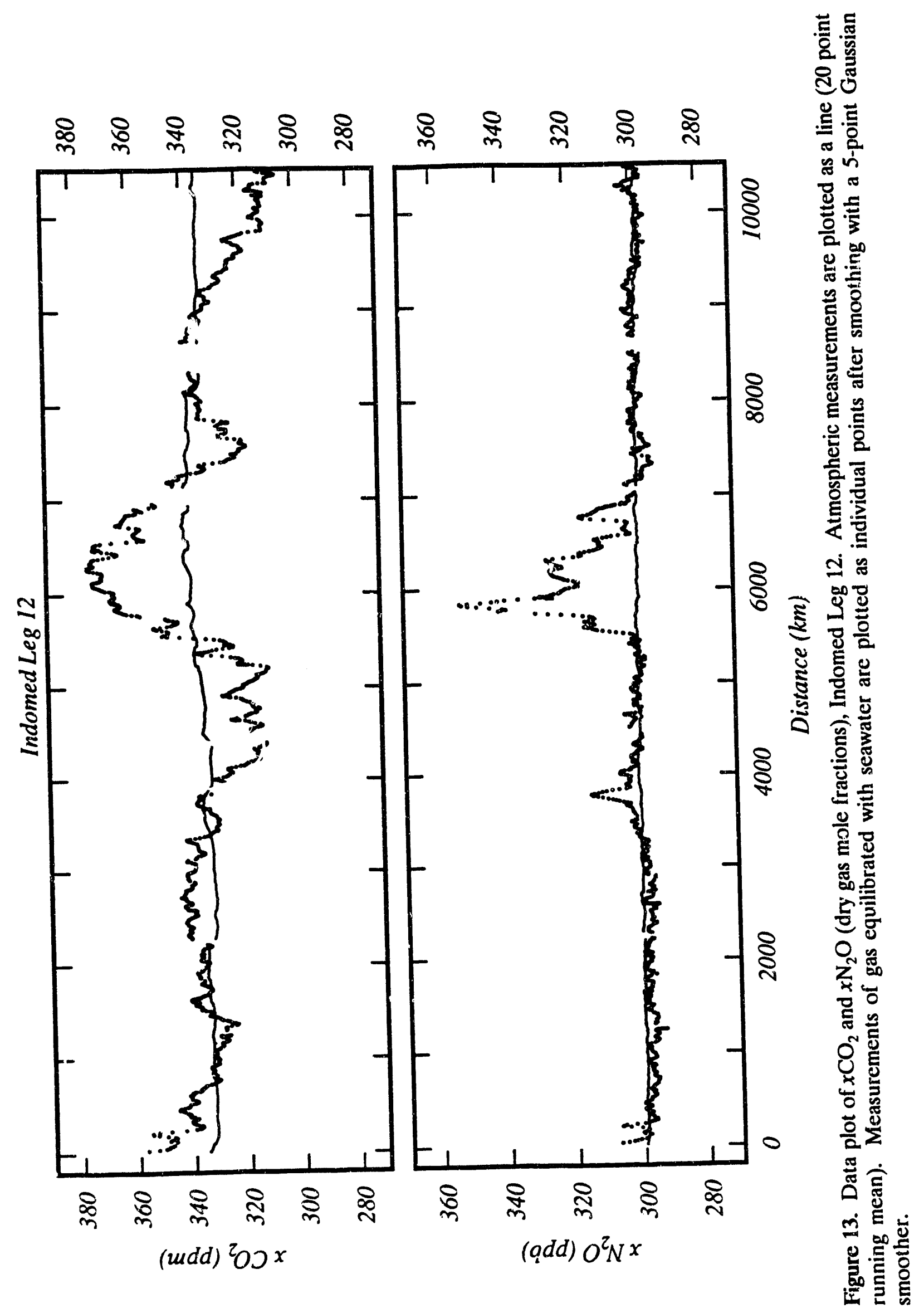




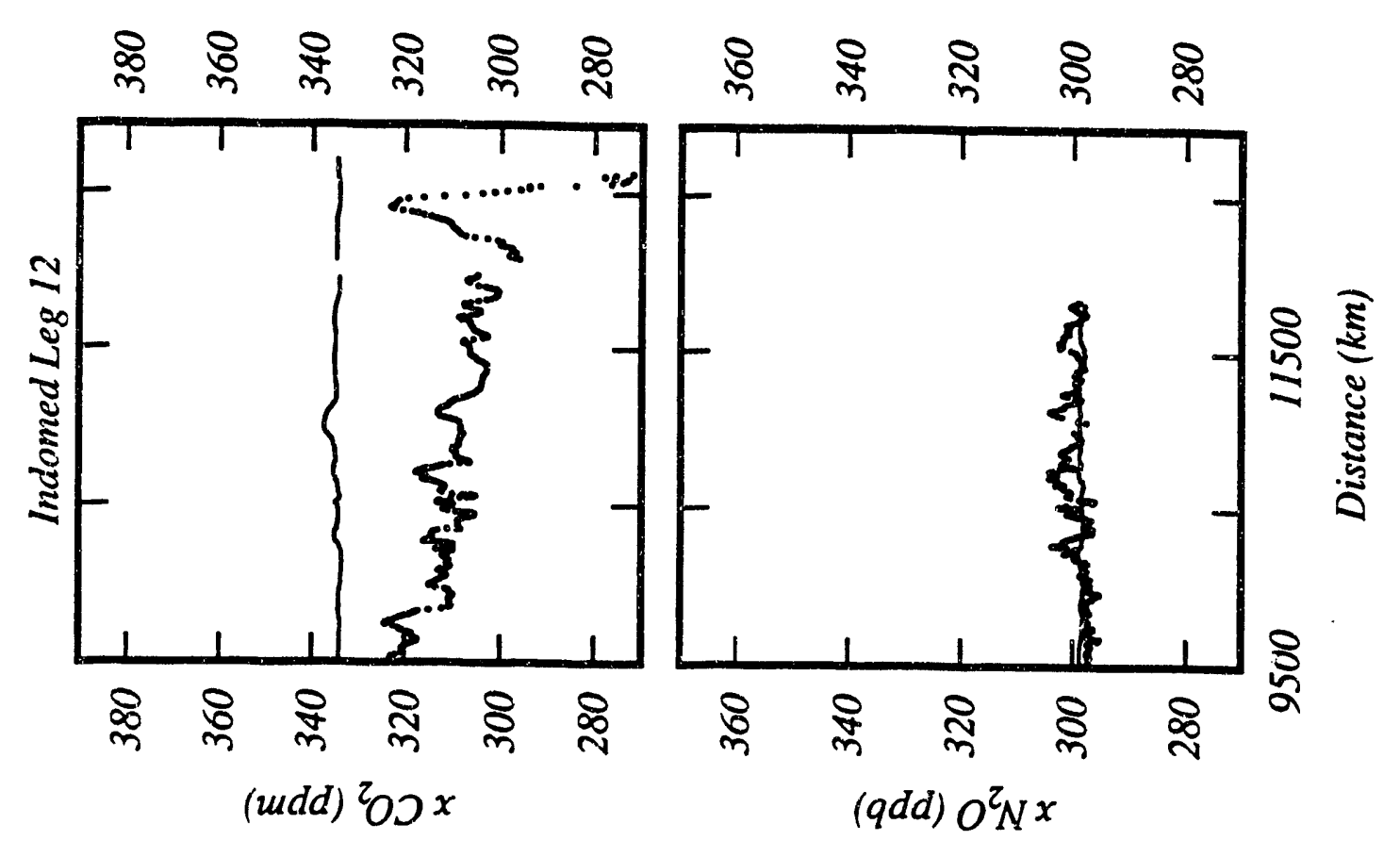

己 


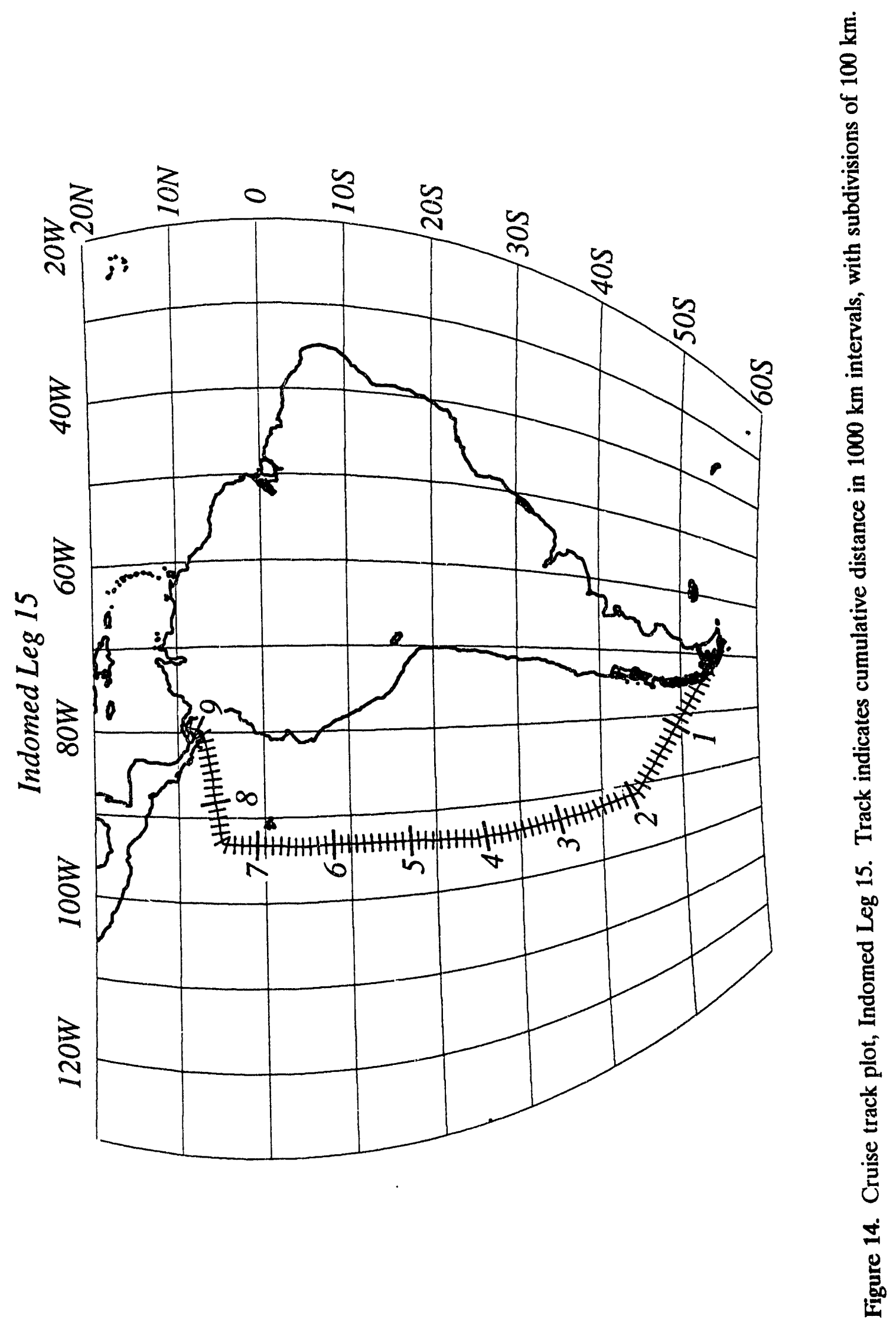




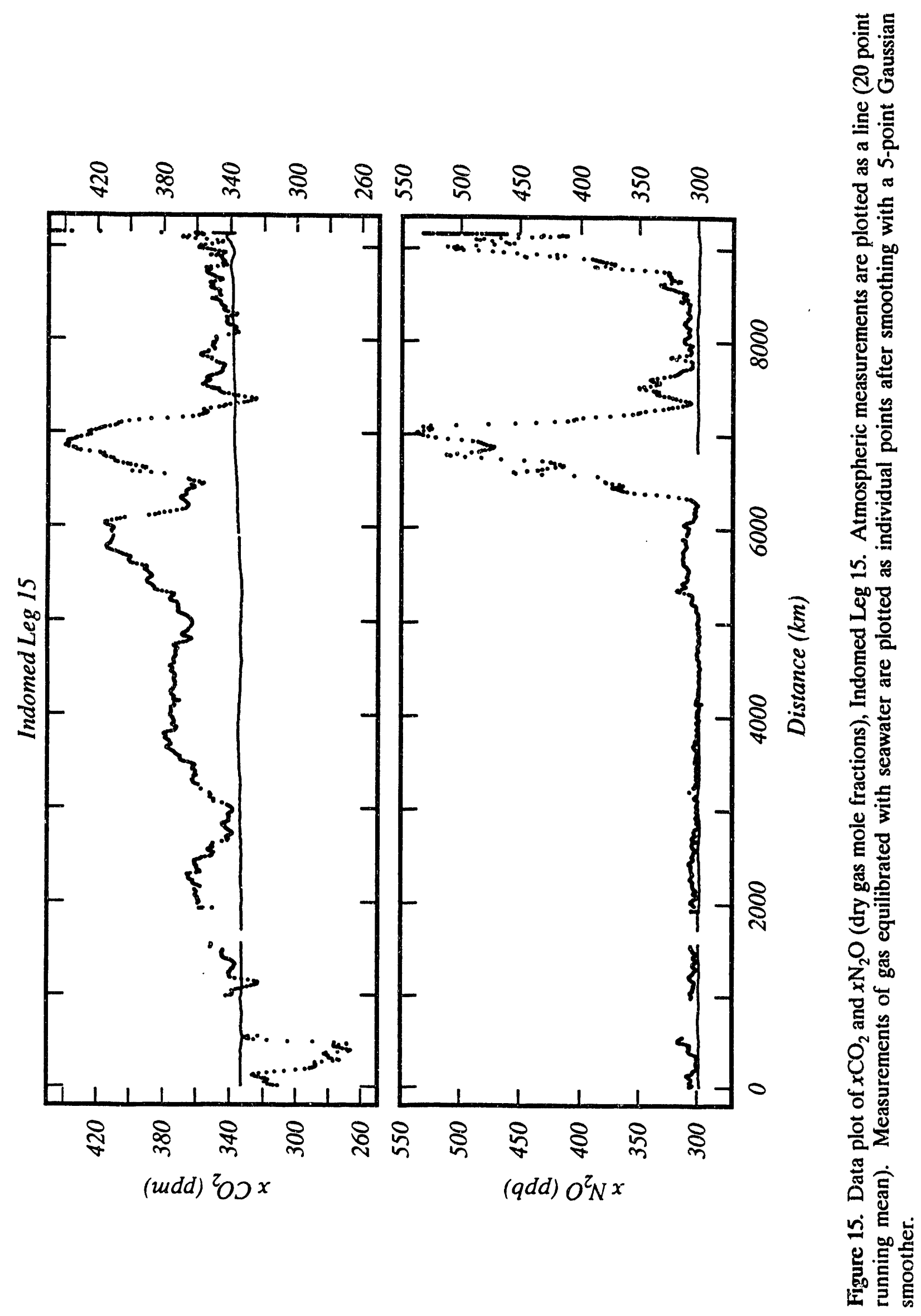




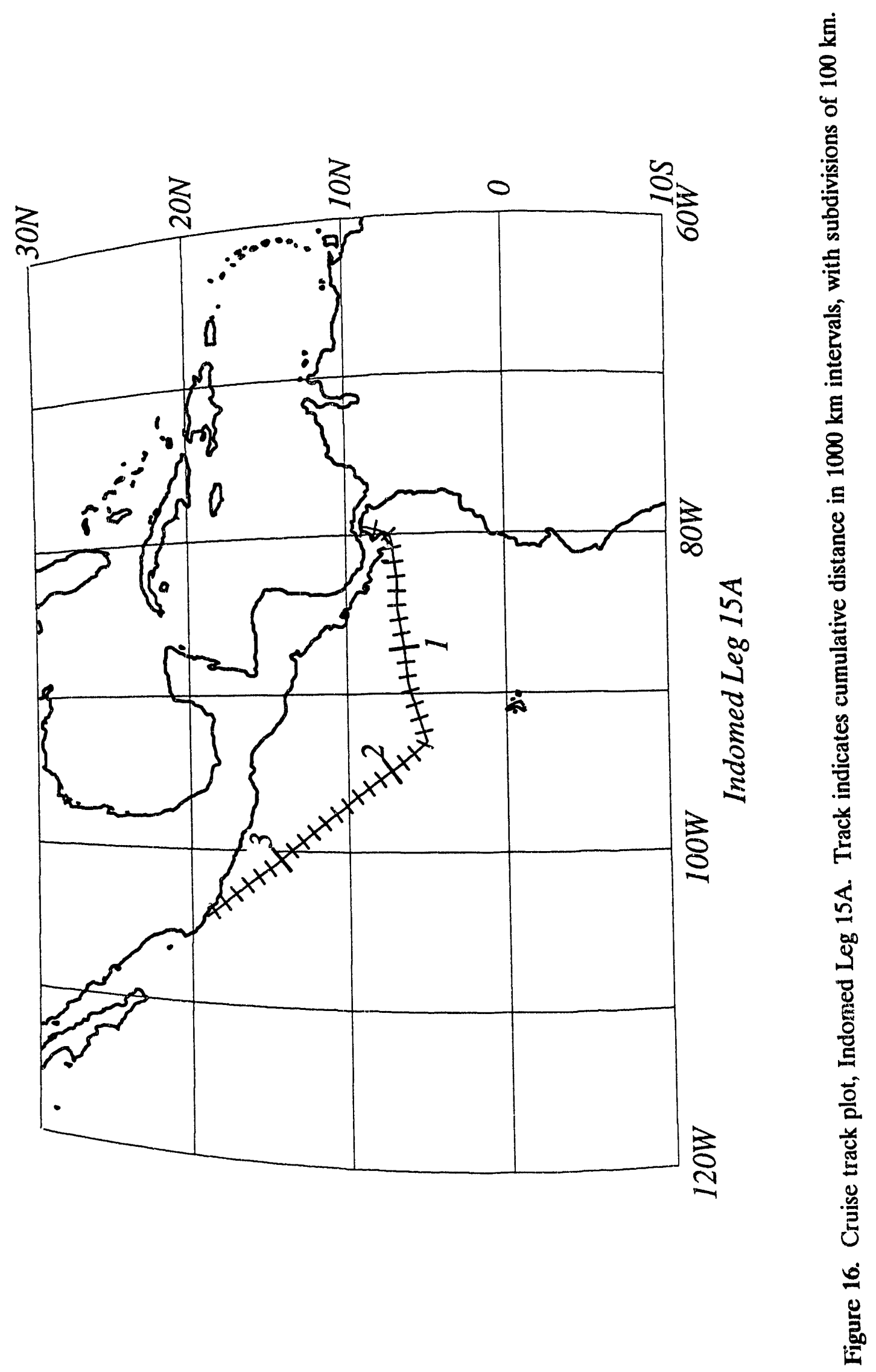




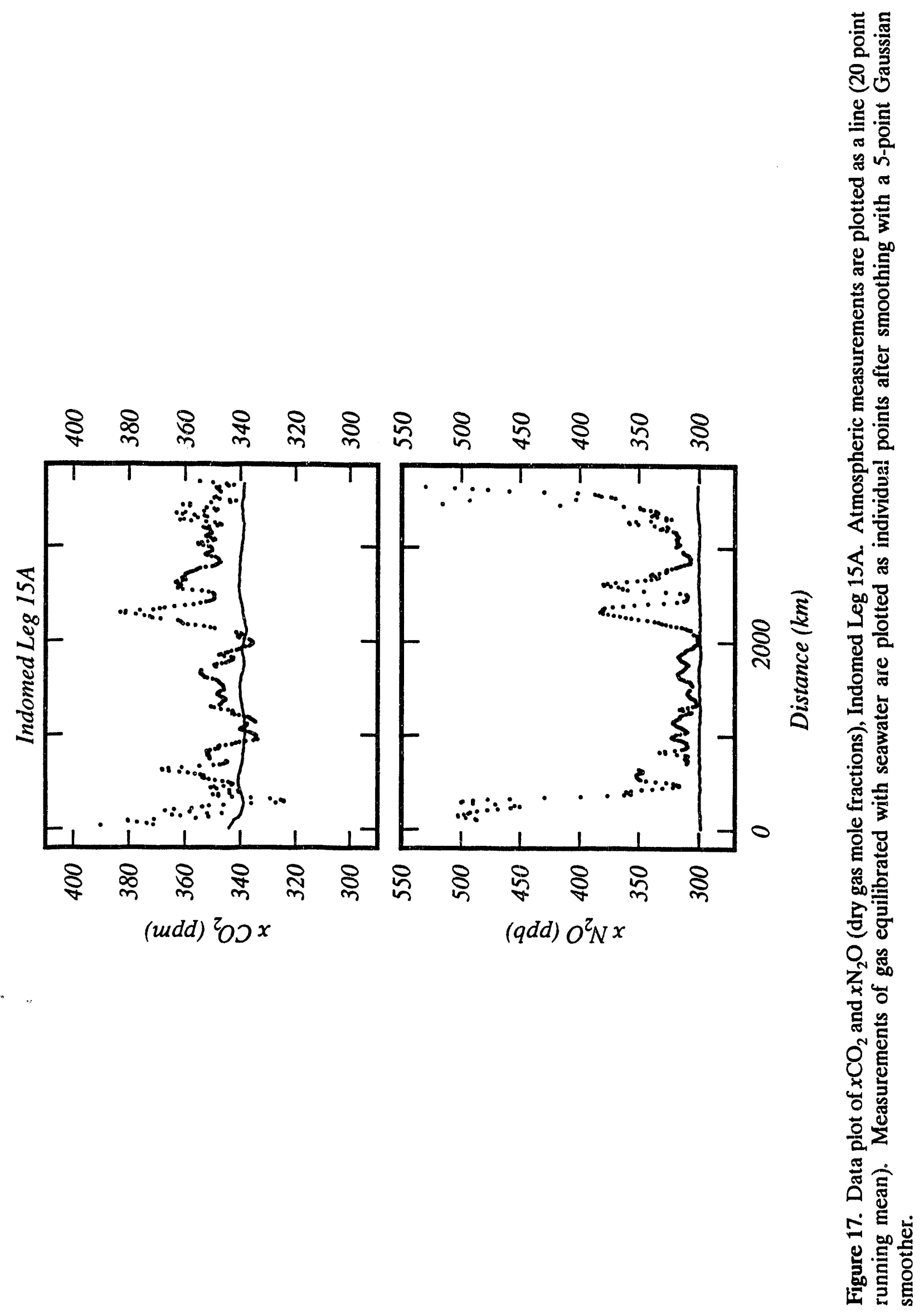




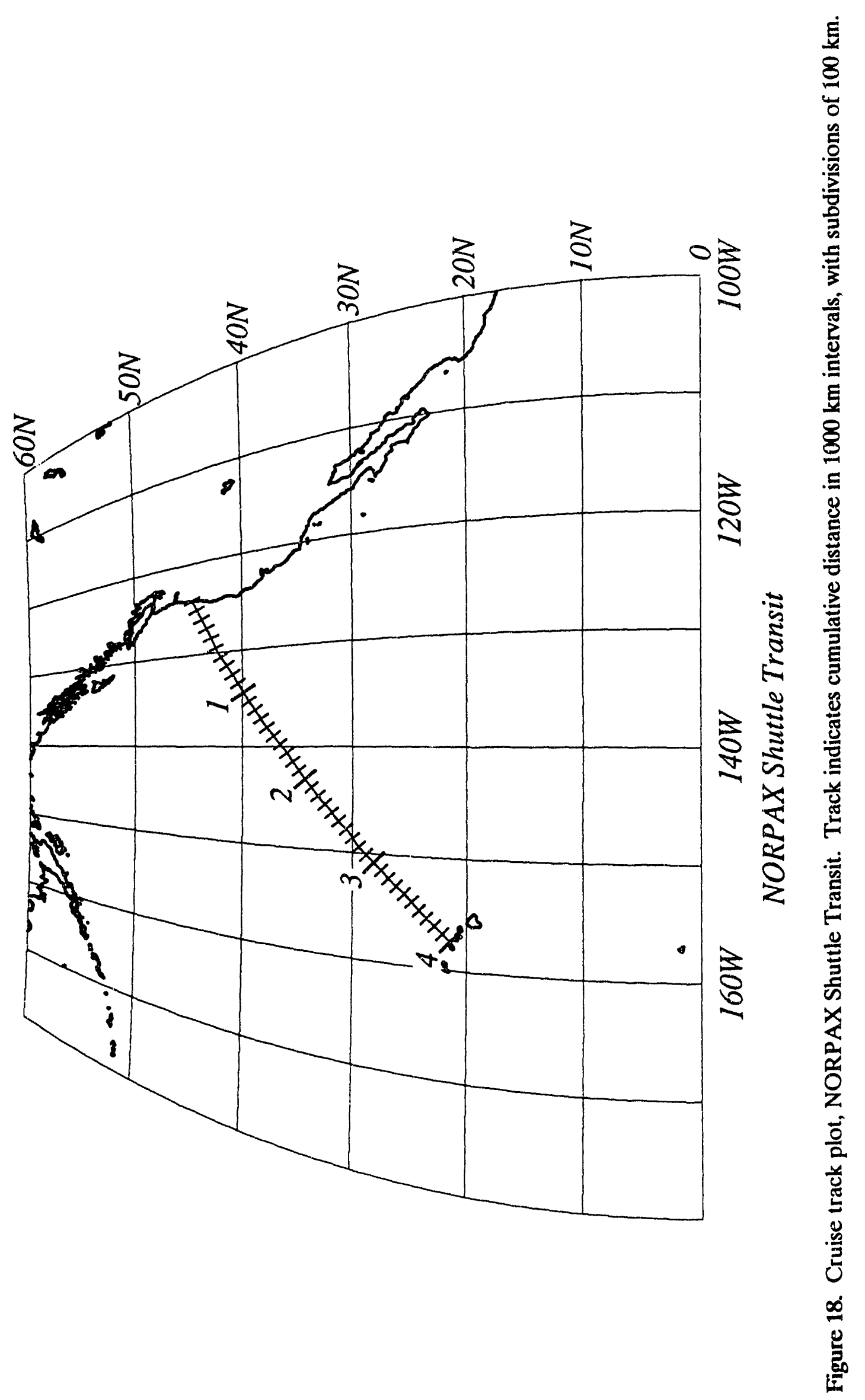




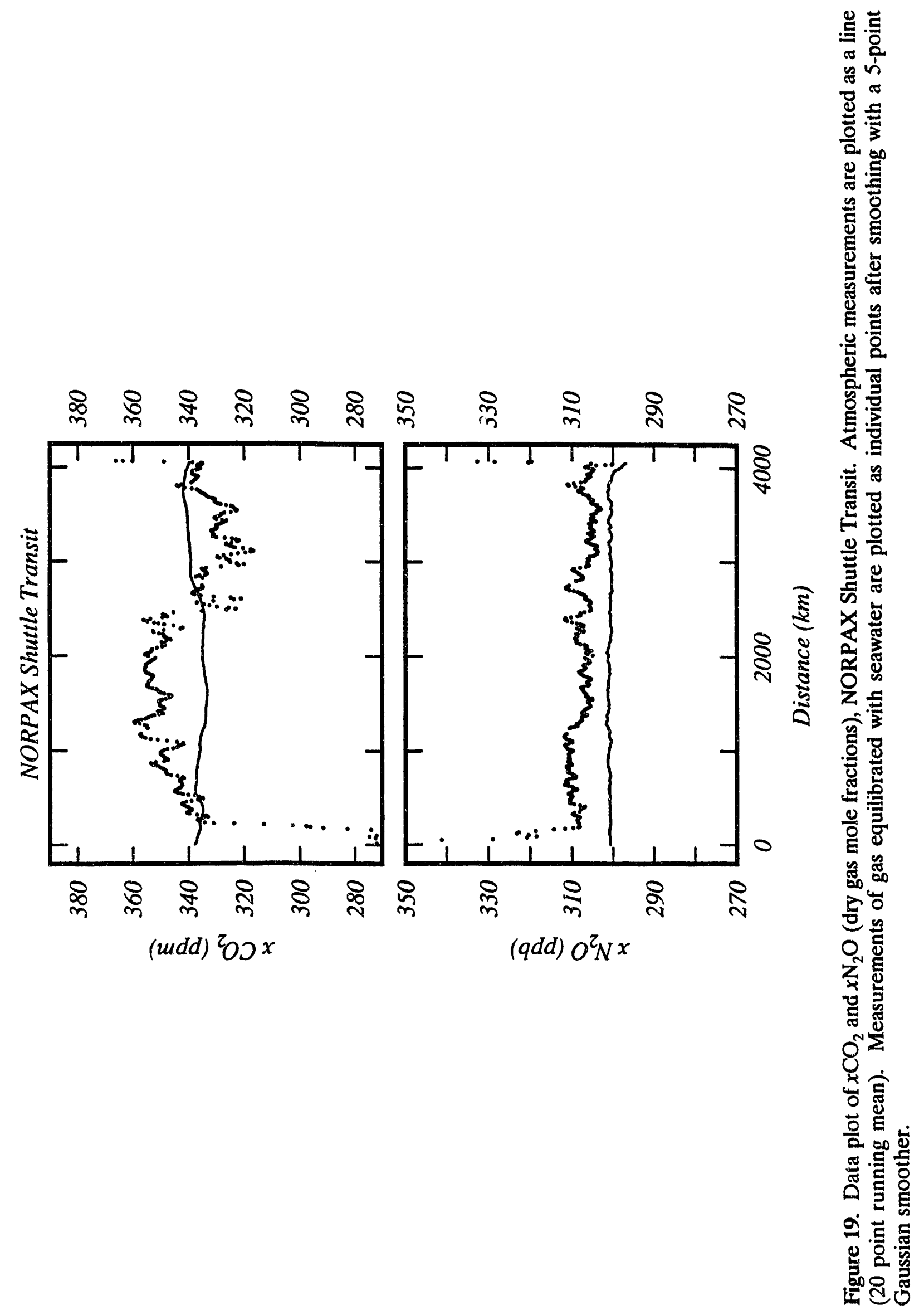




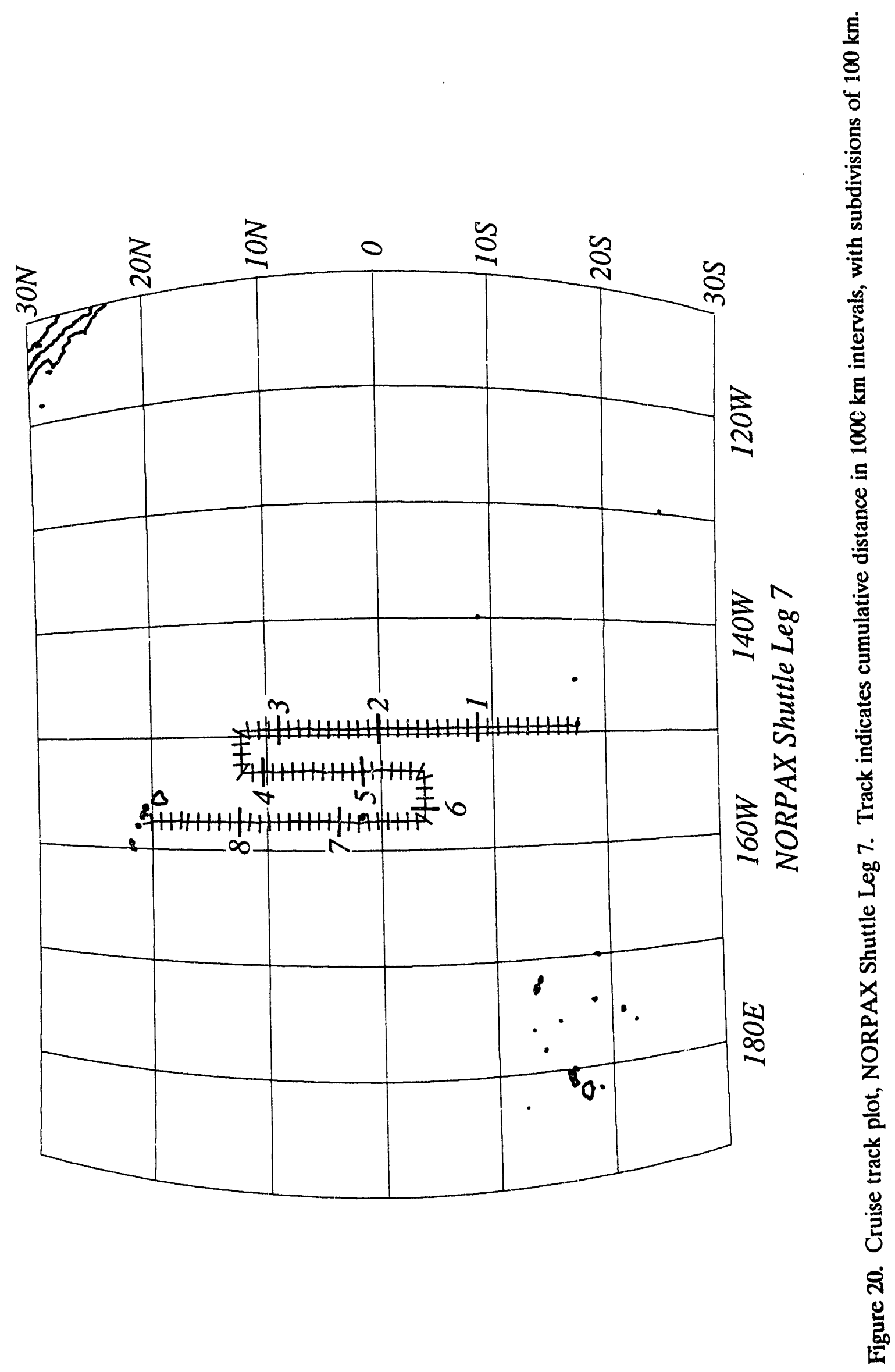




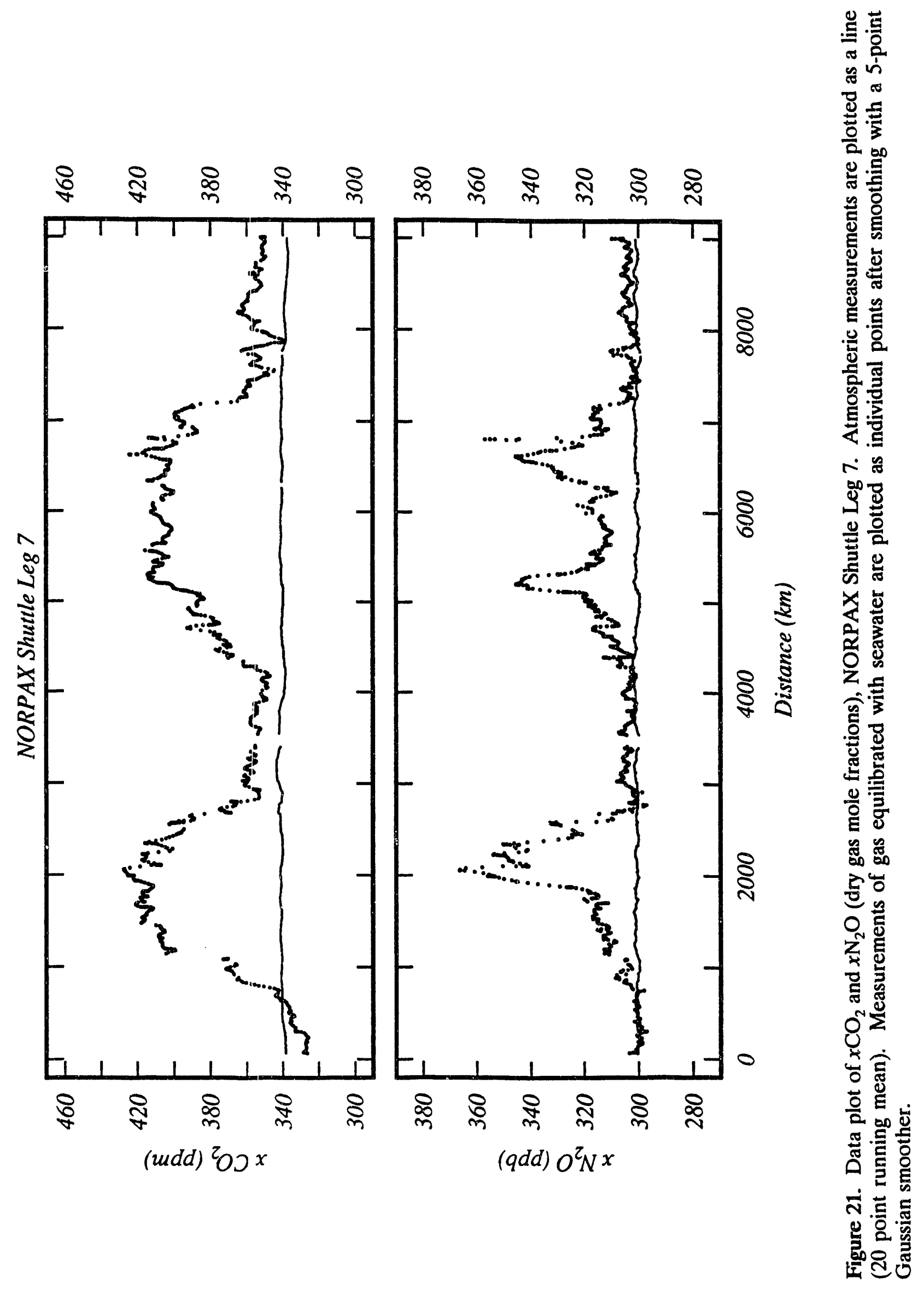




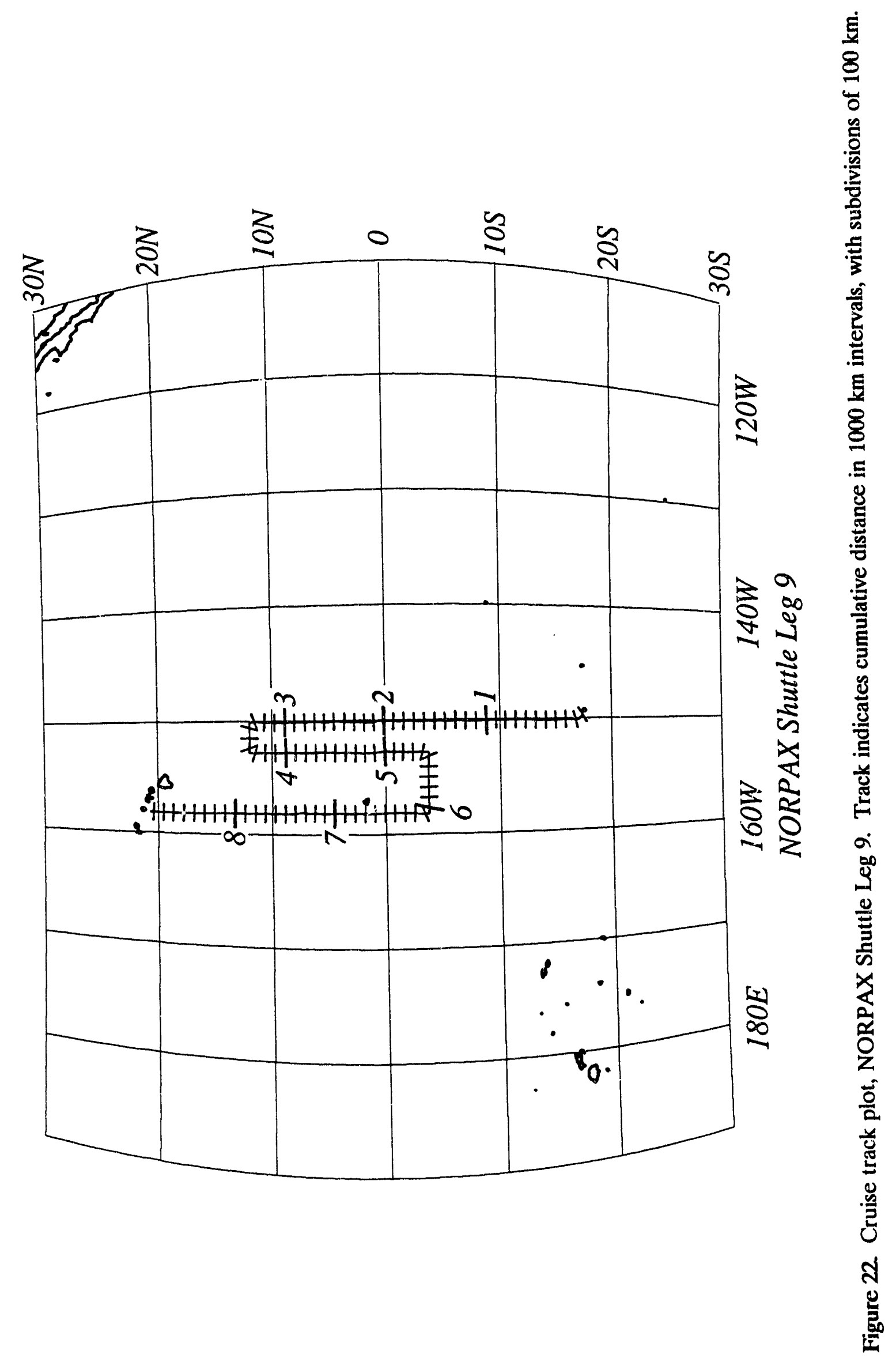




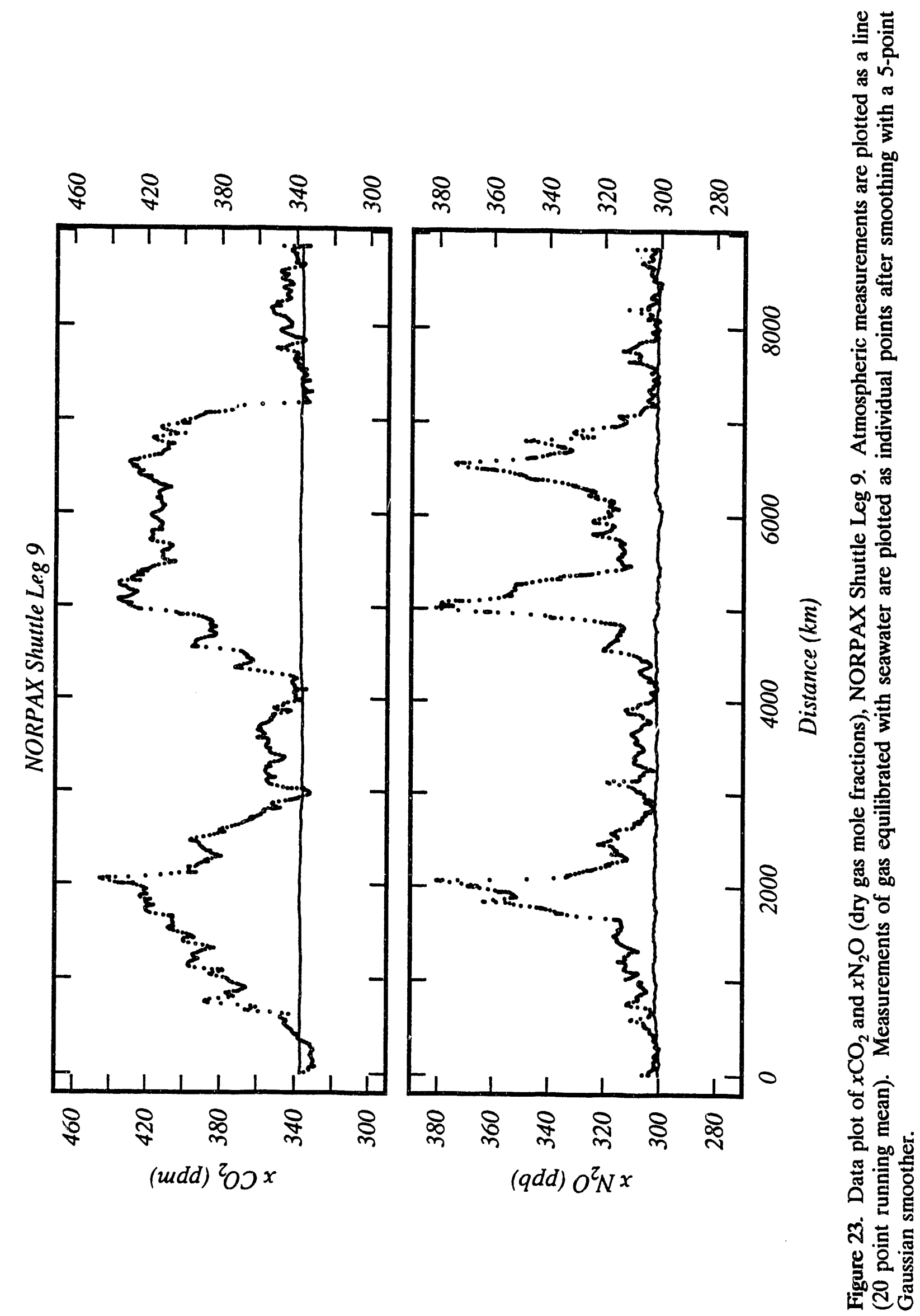




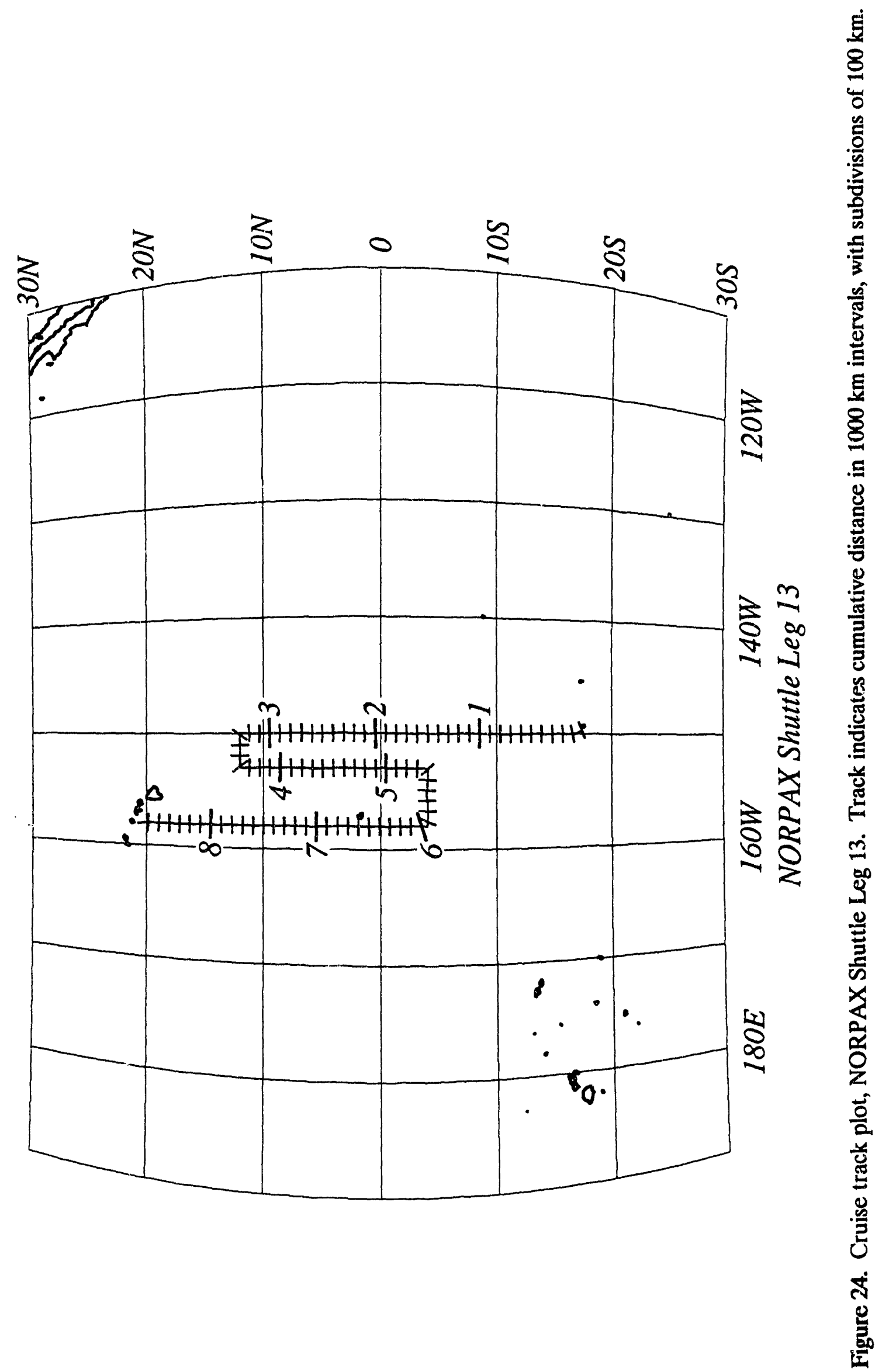




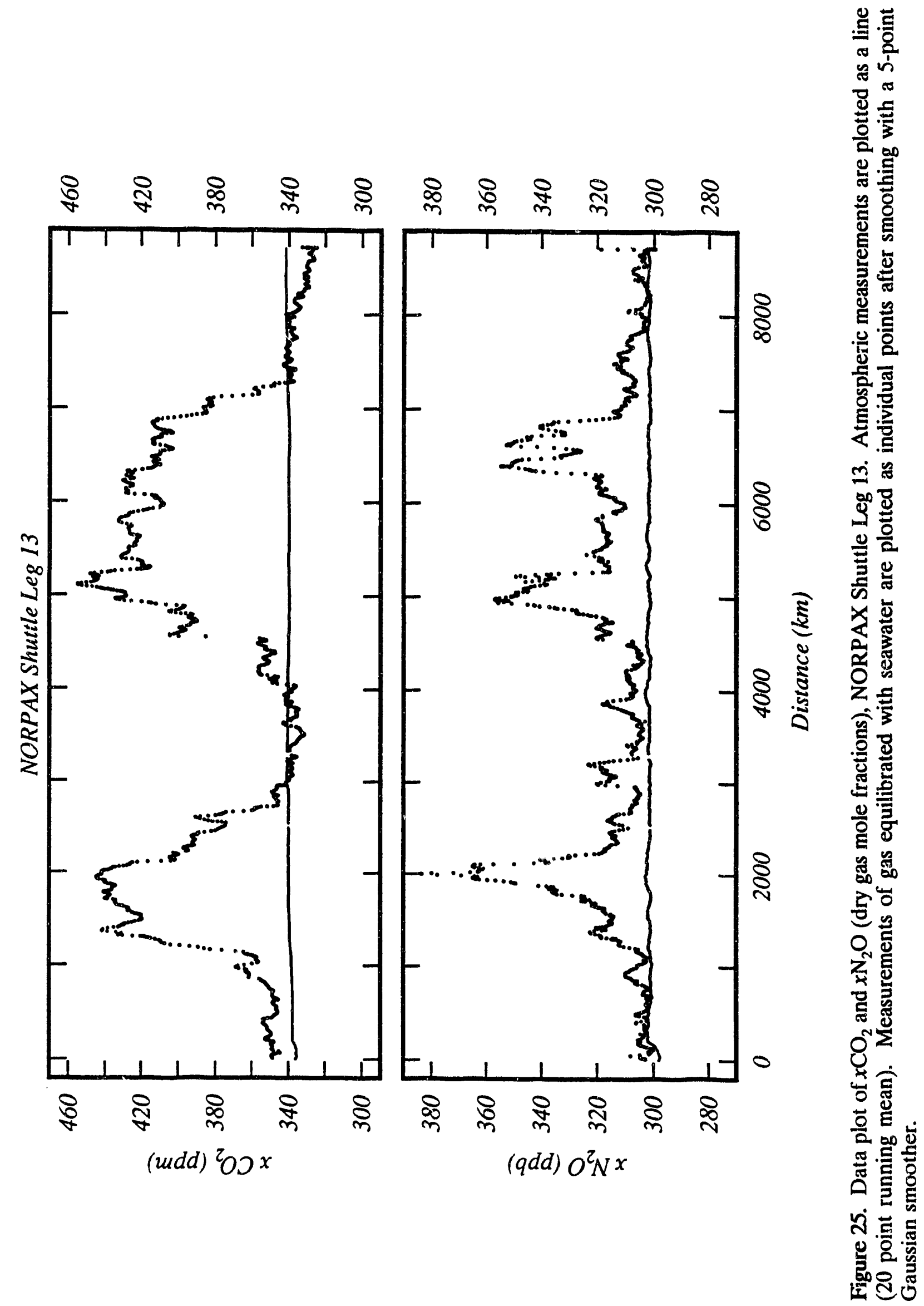




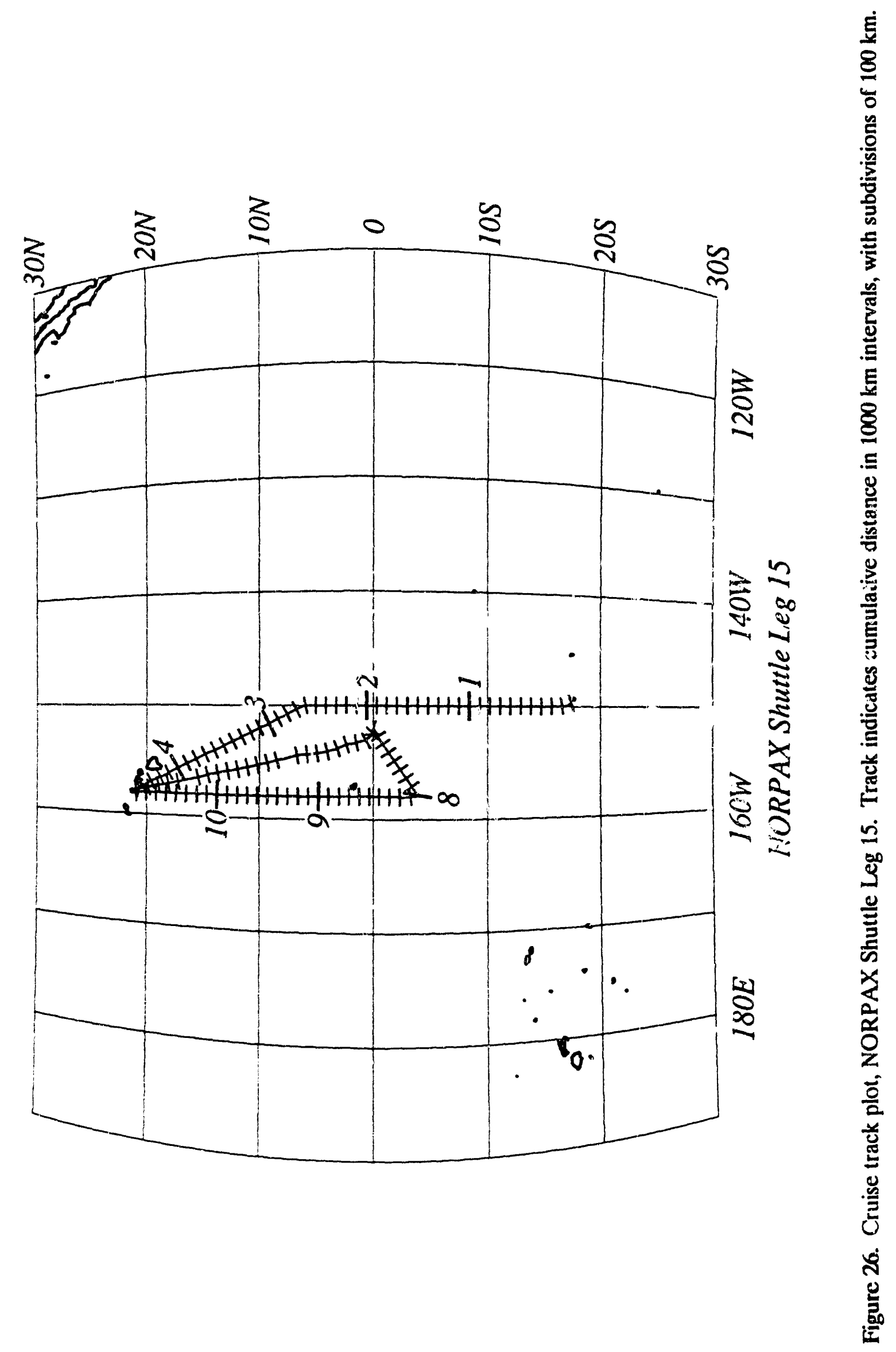




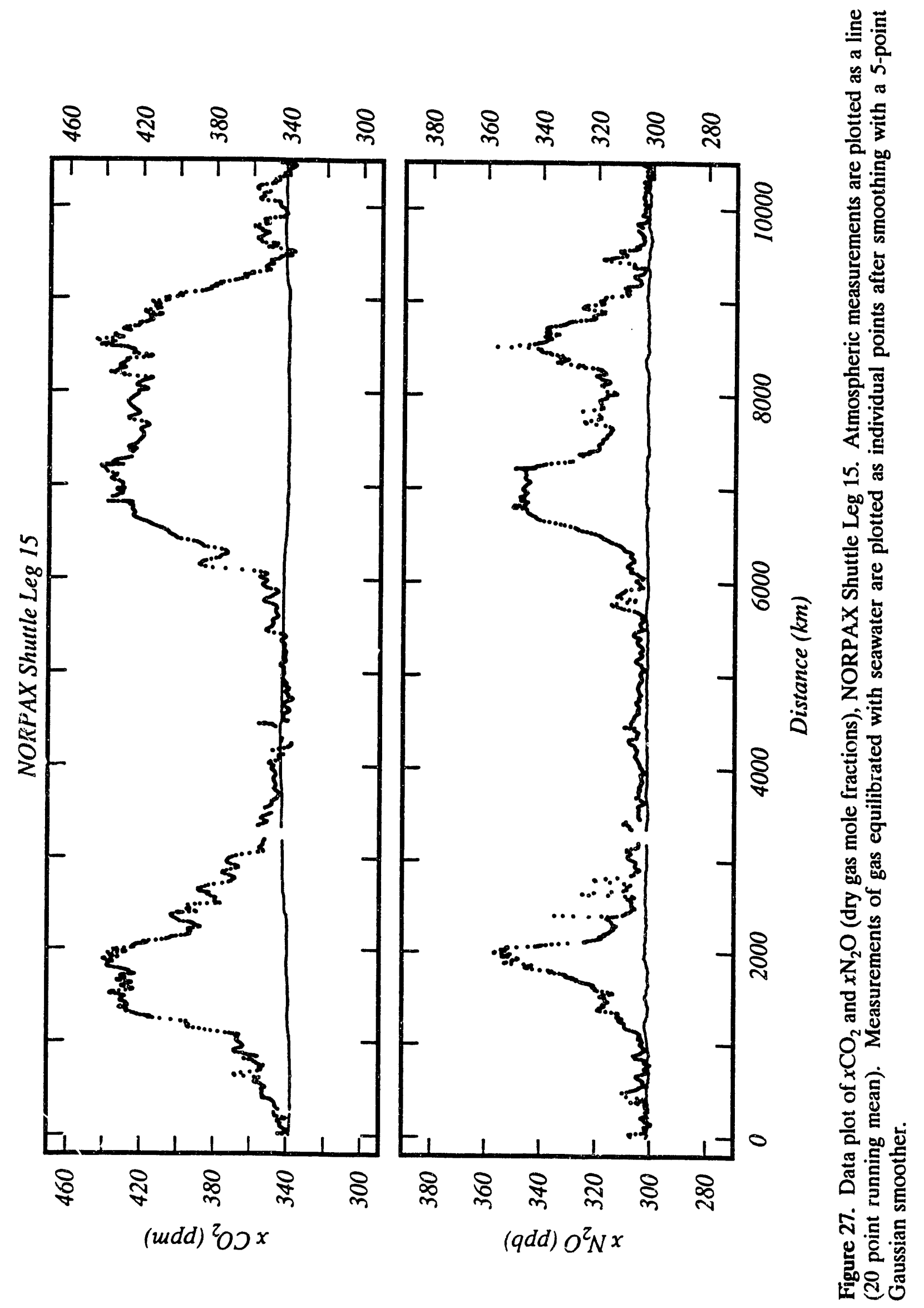




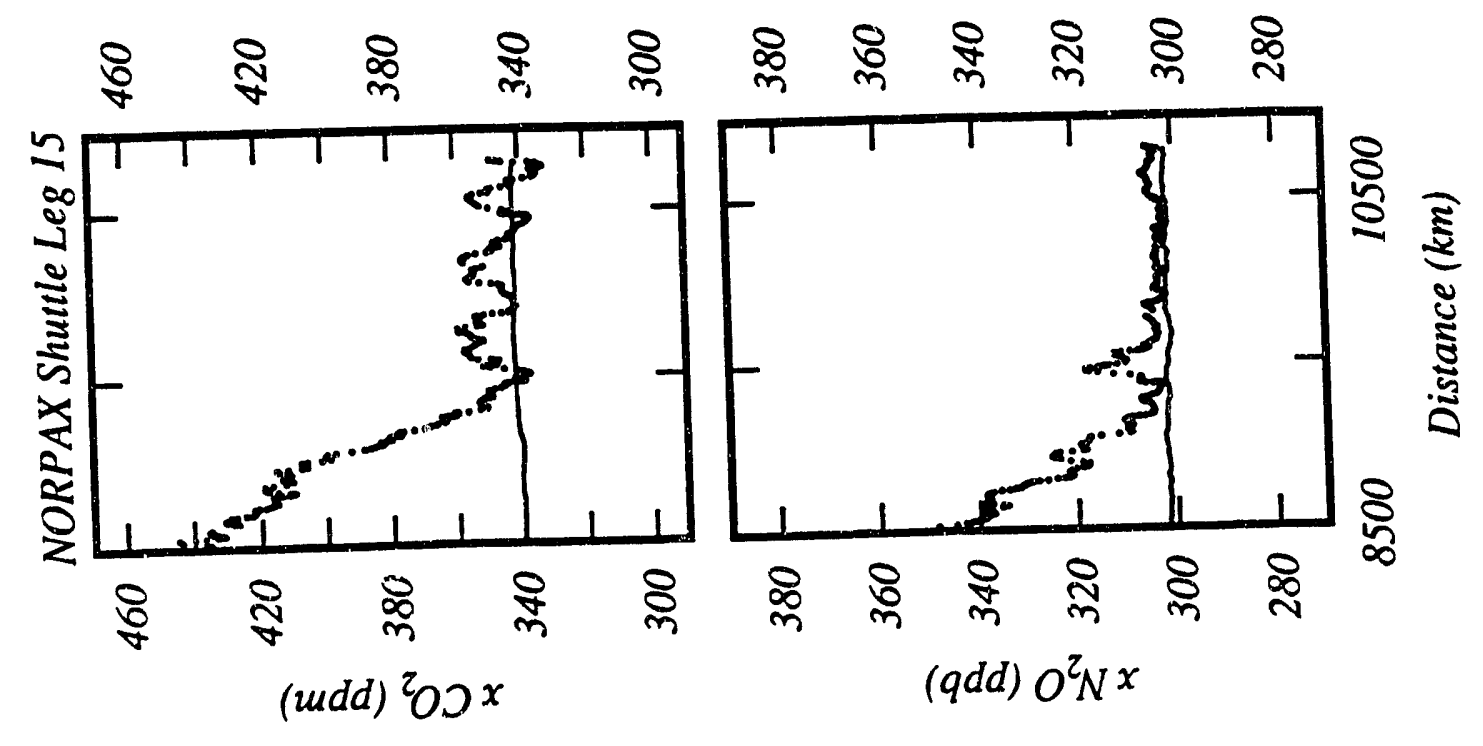

苞 


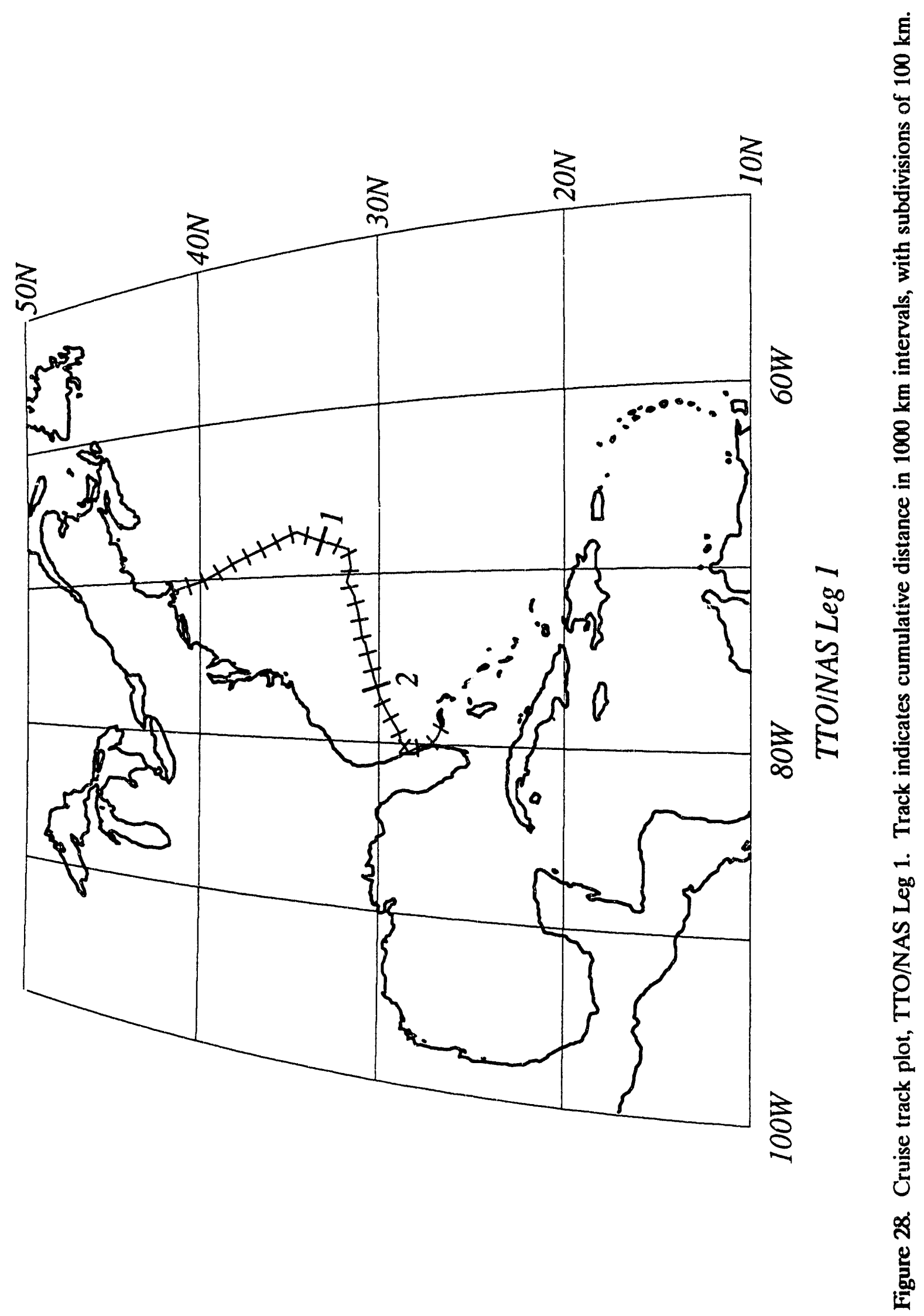




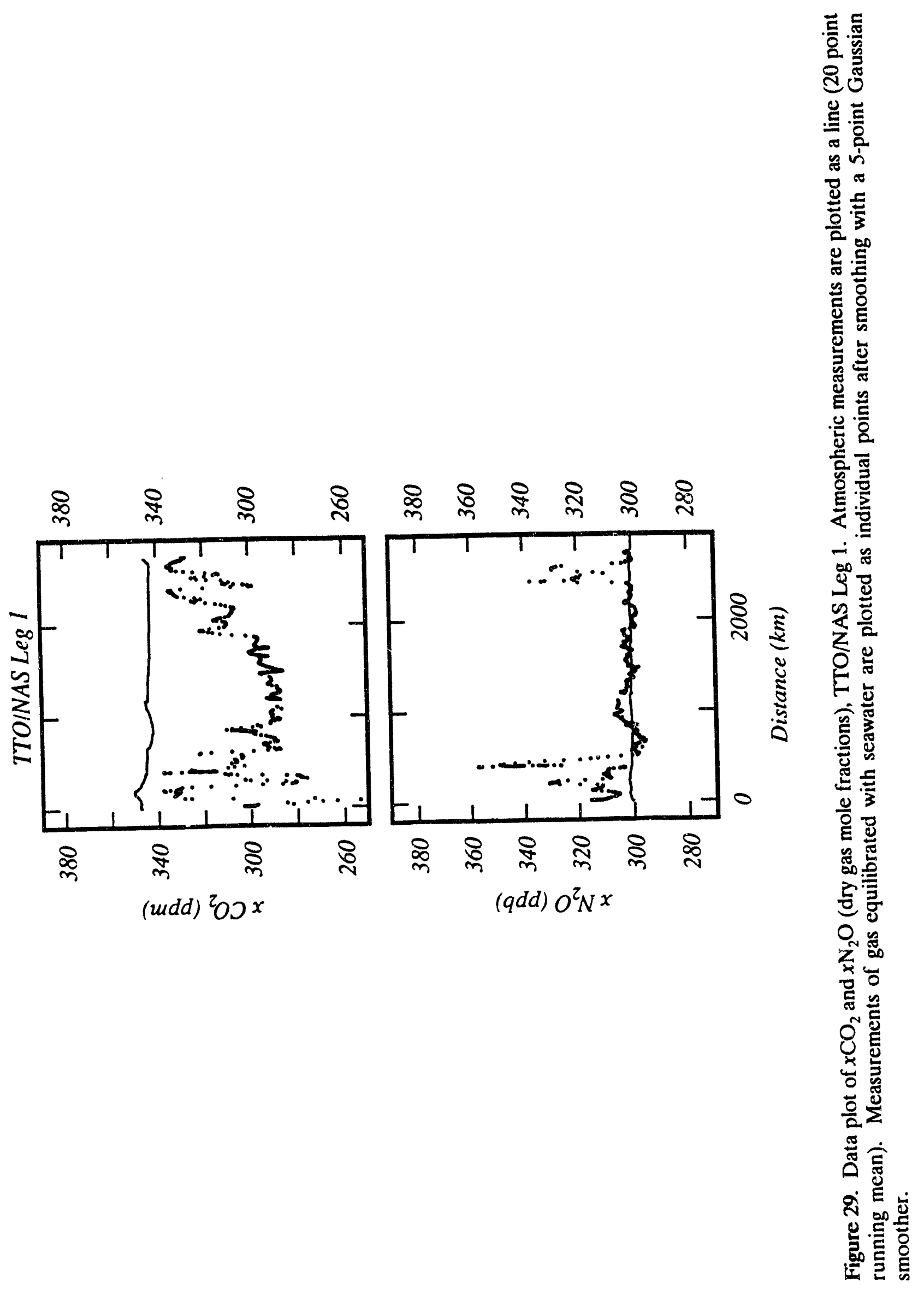




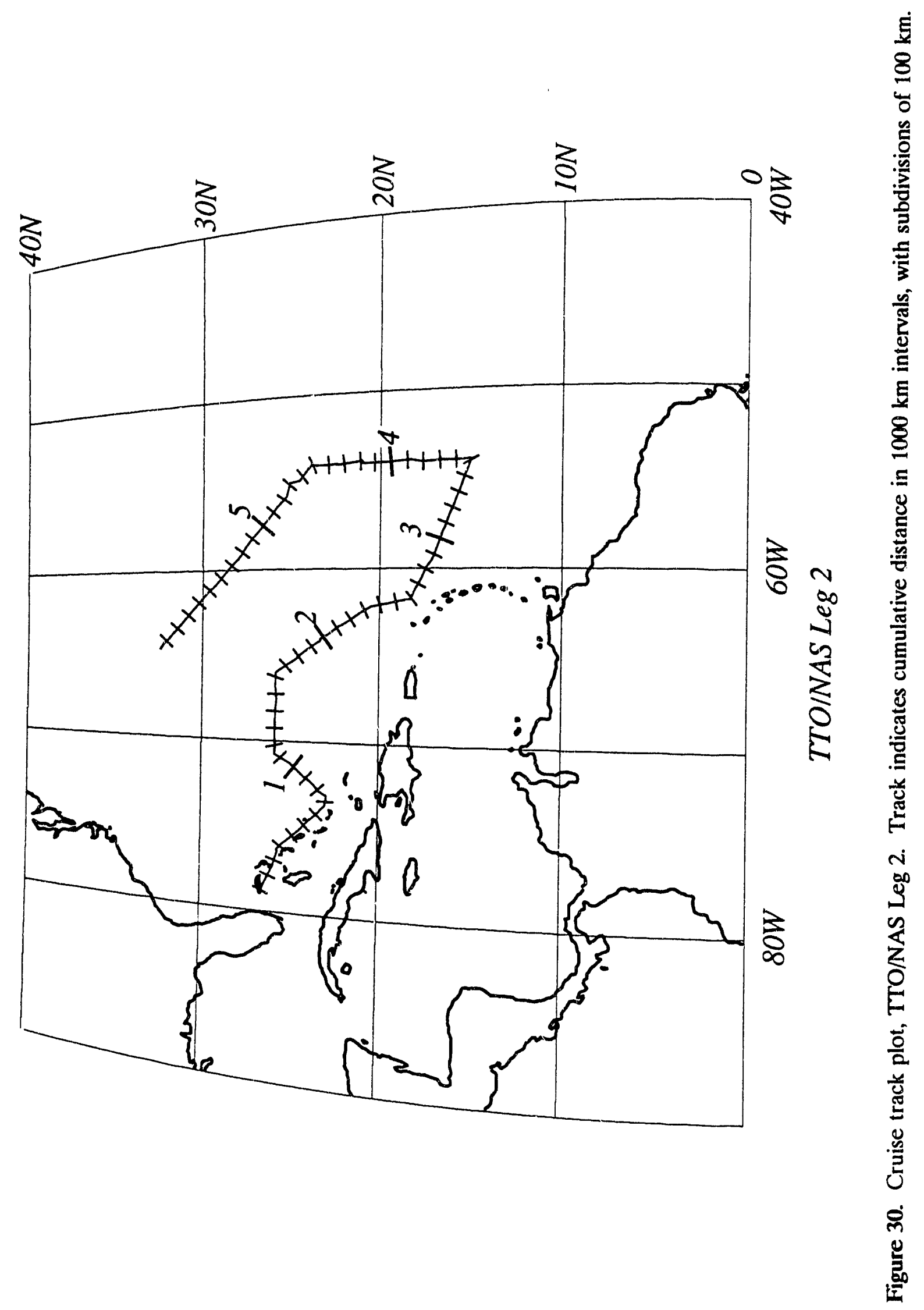




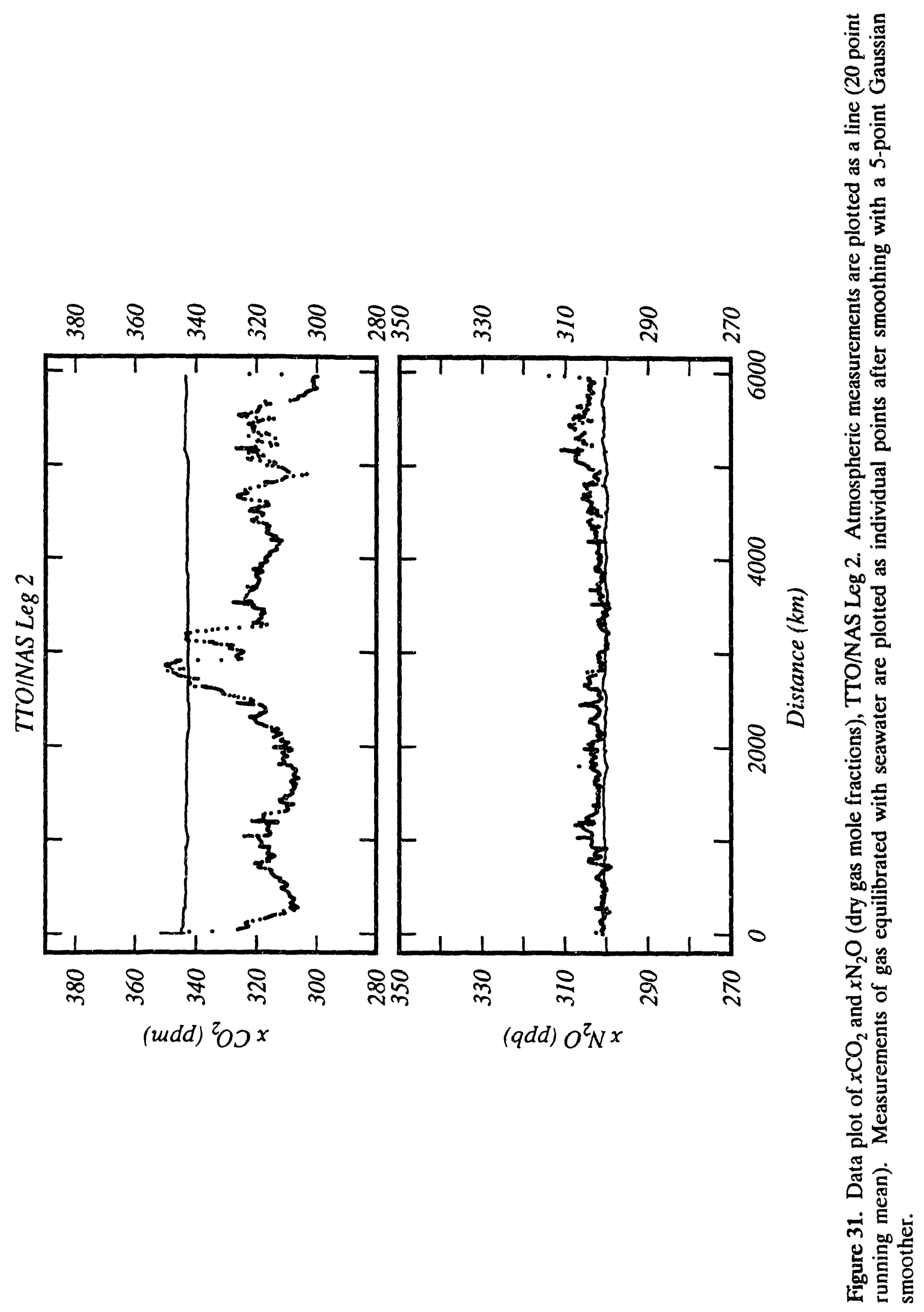




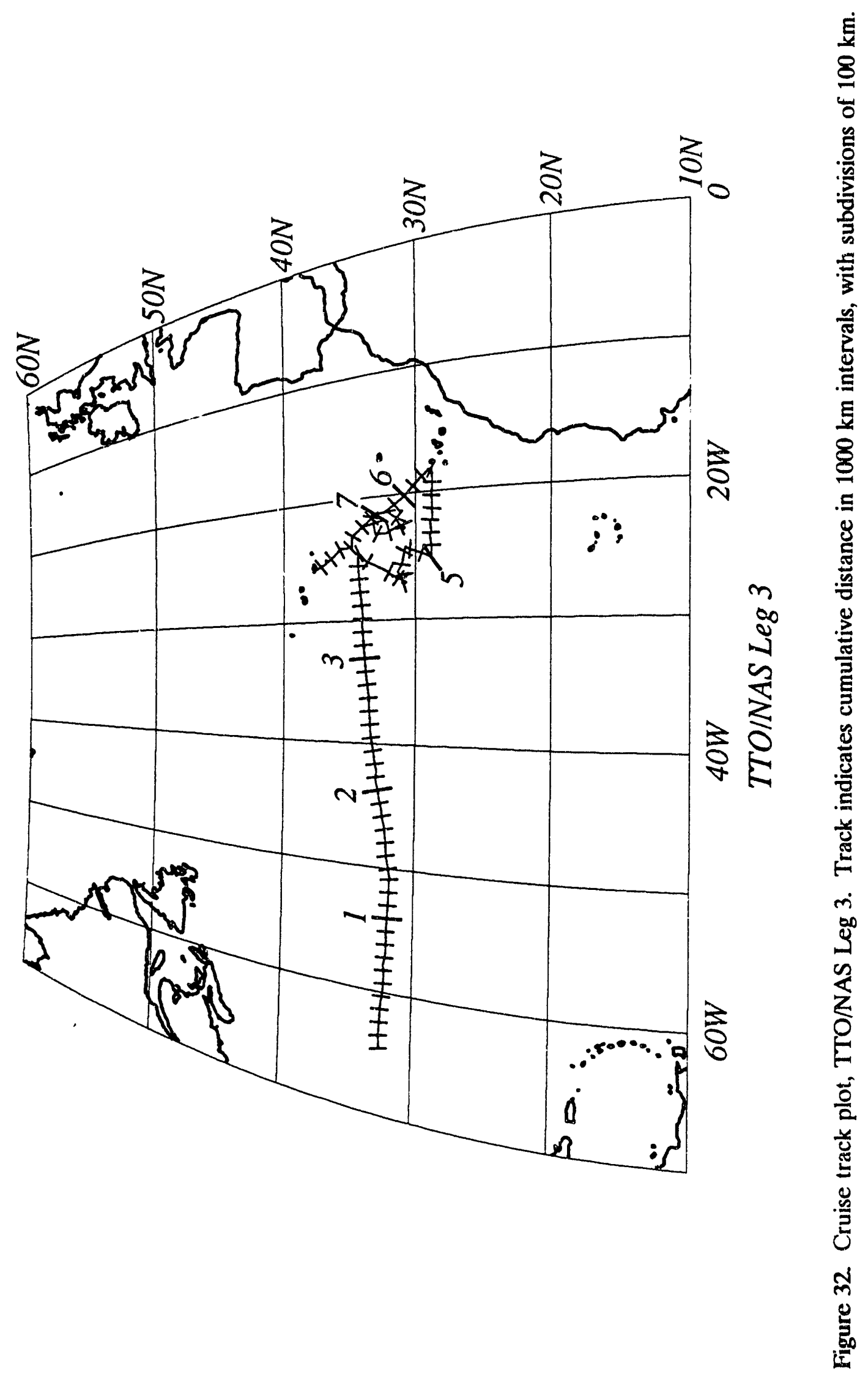




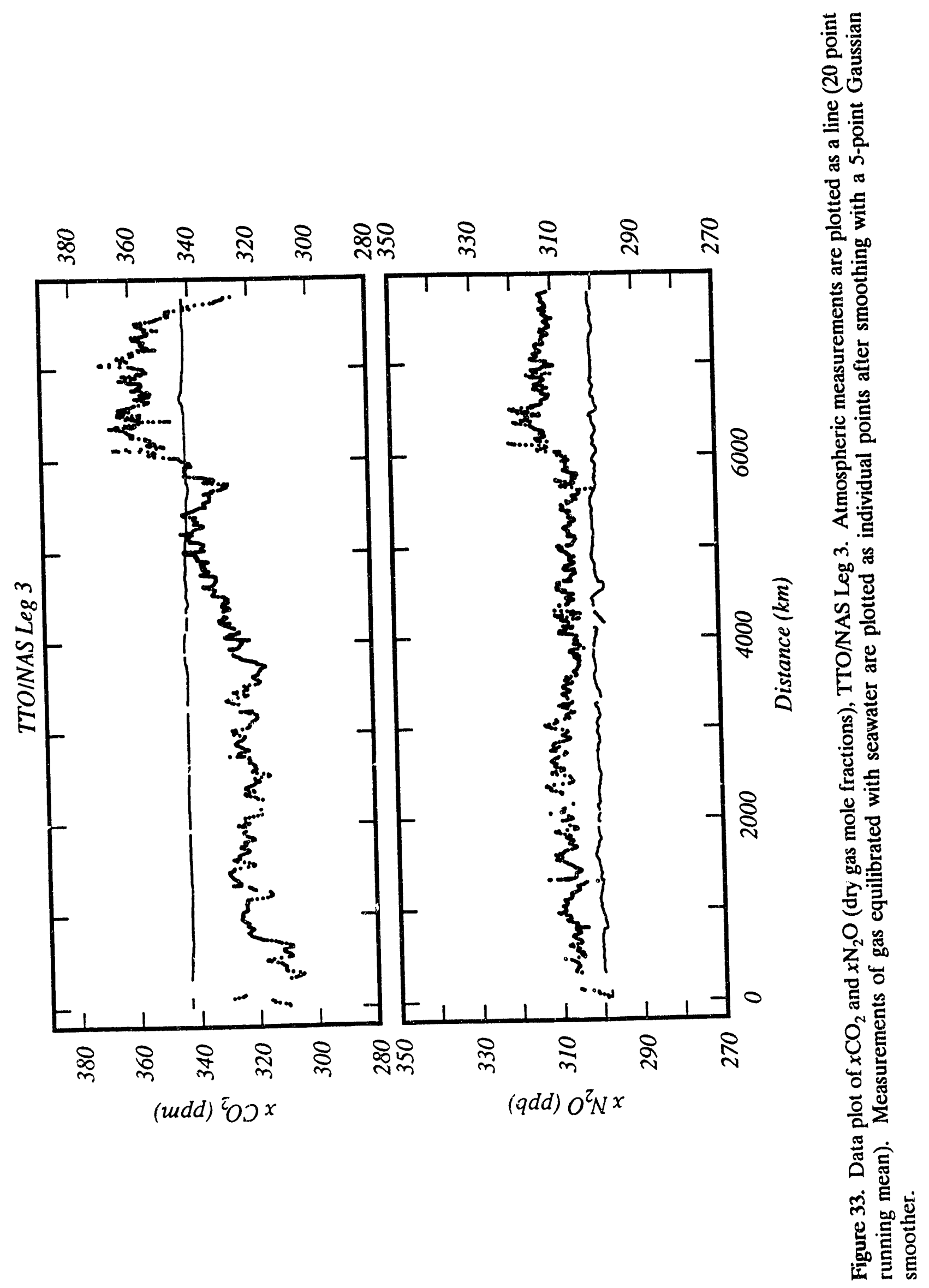




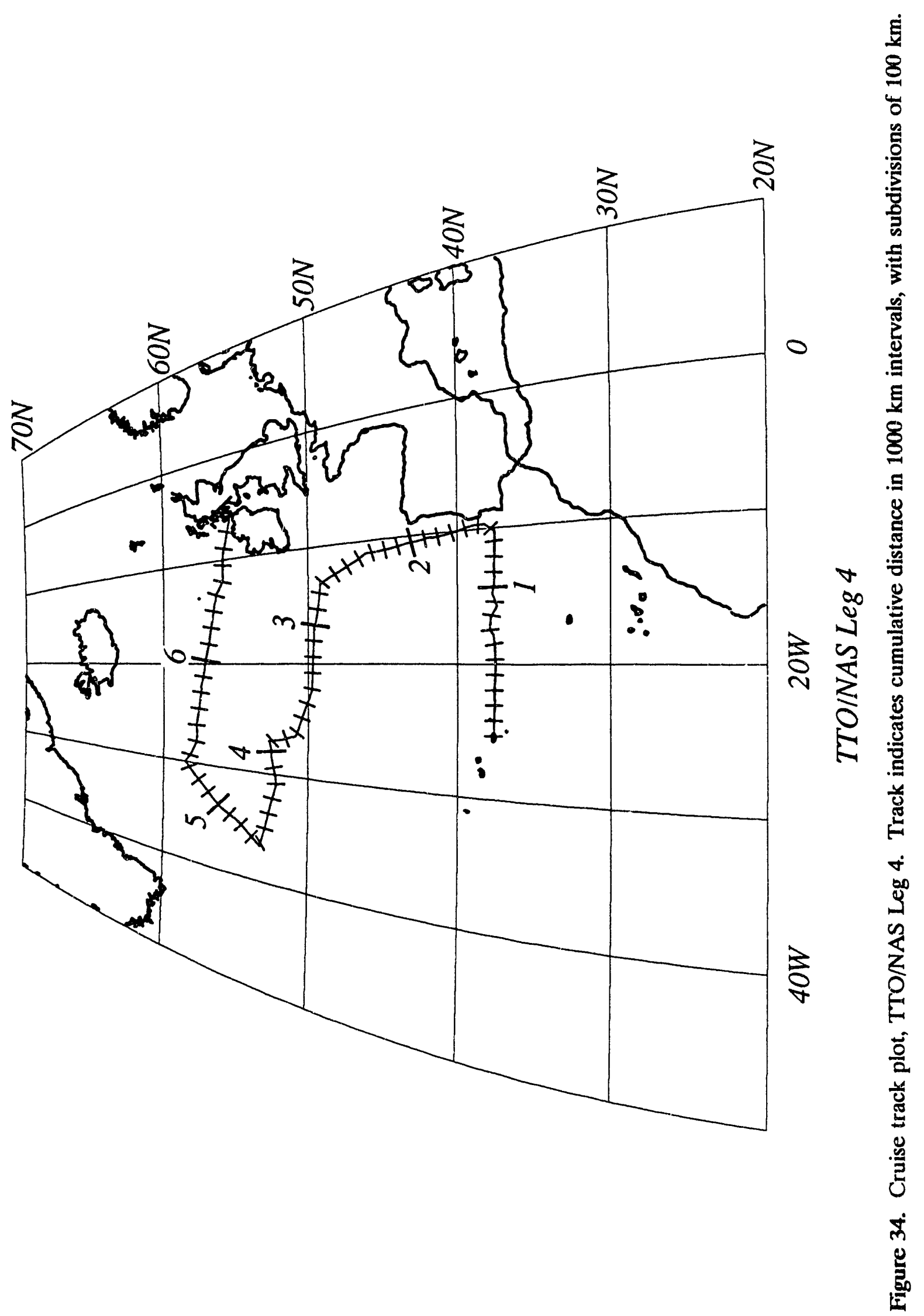




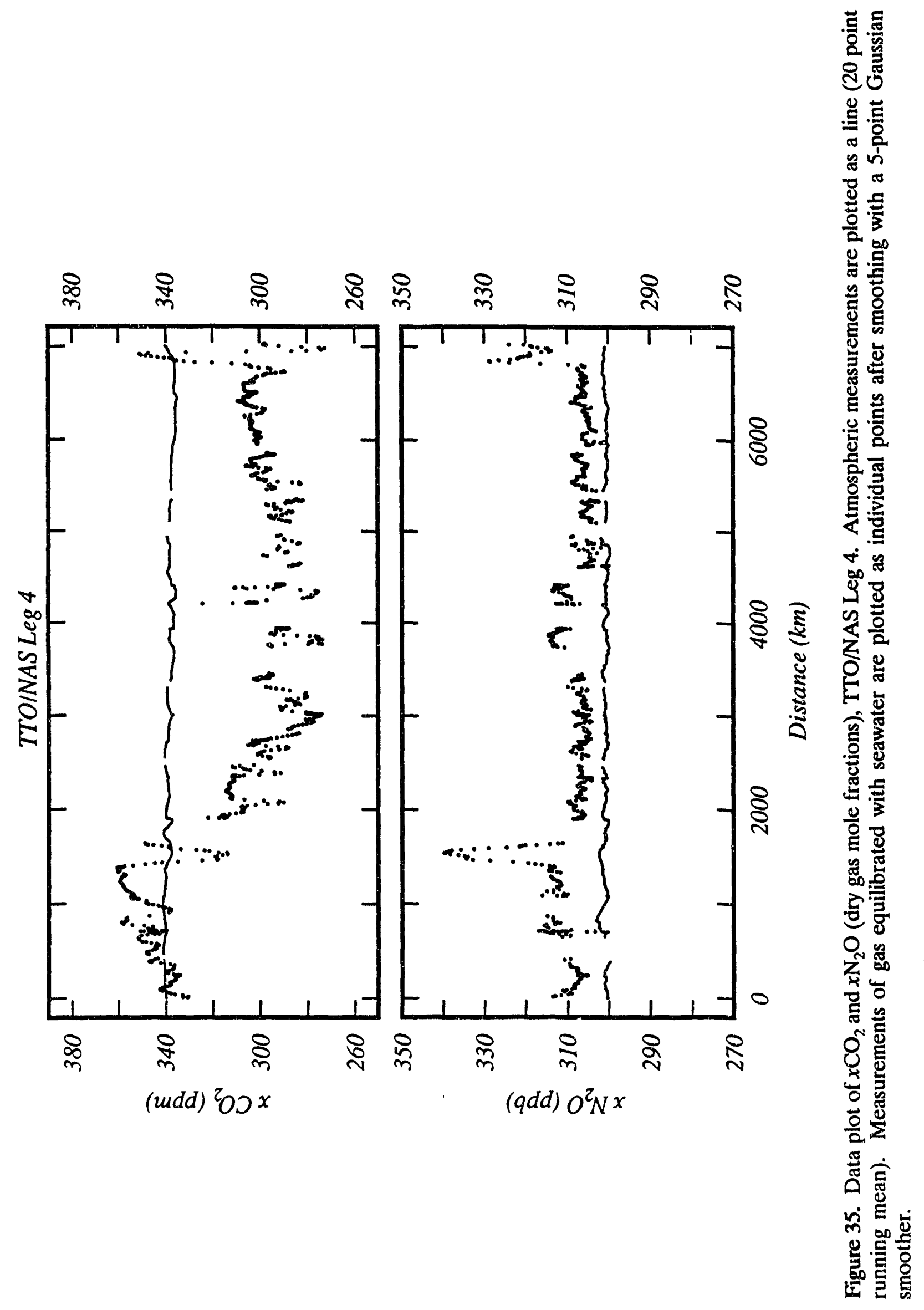




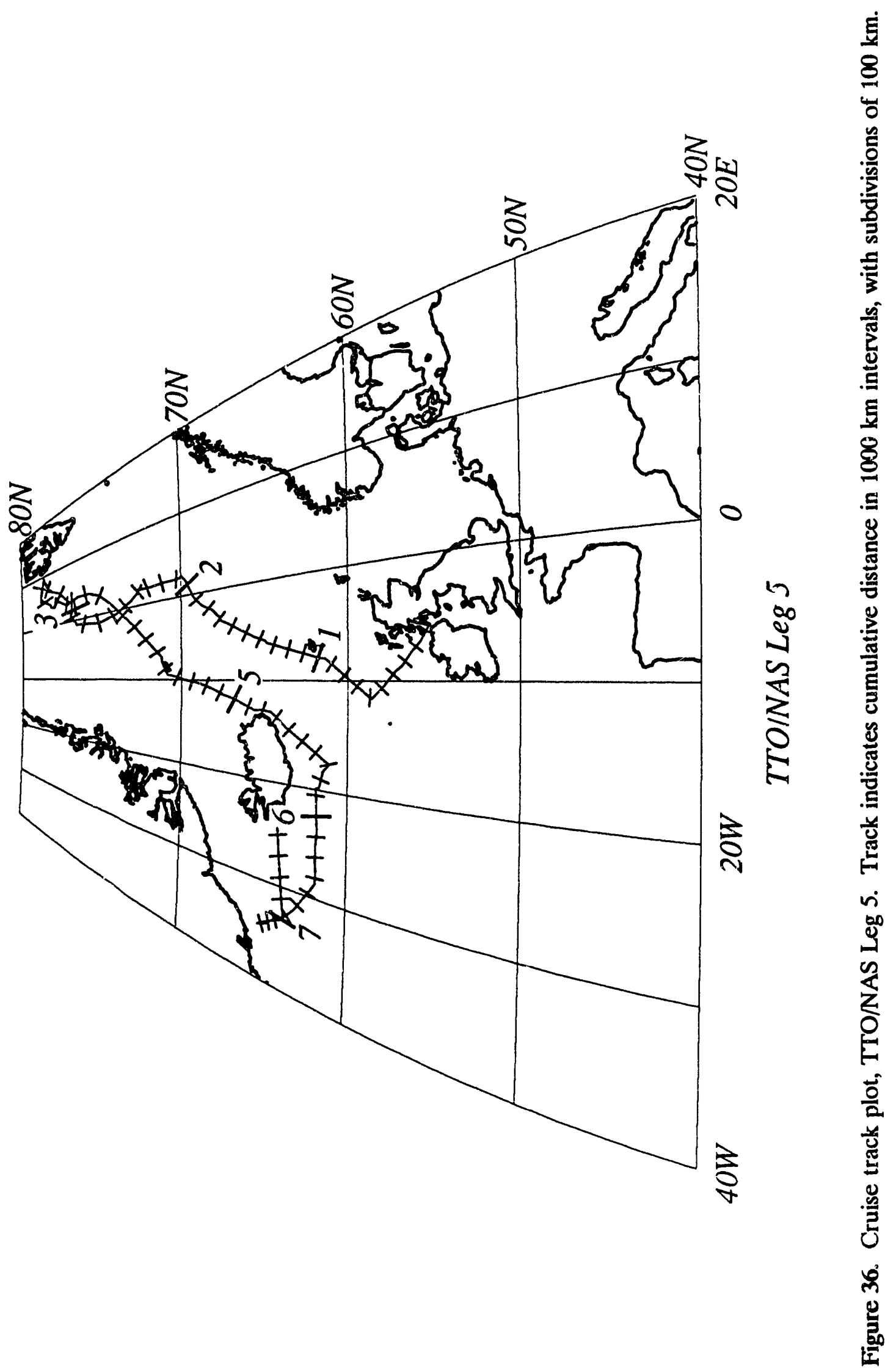




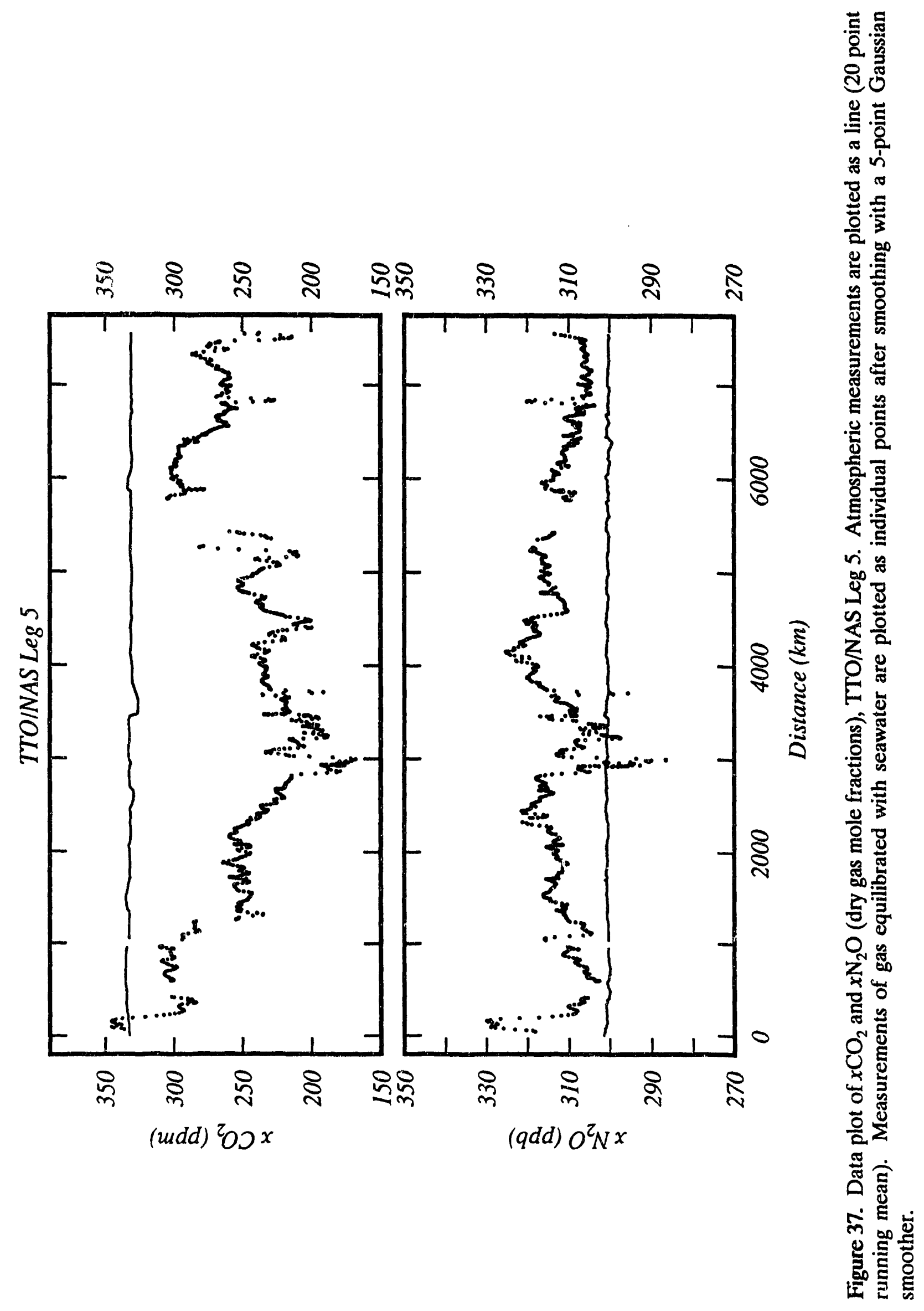




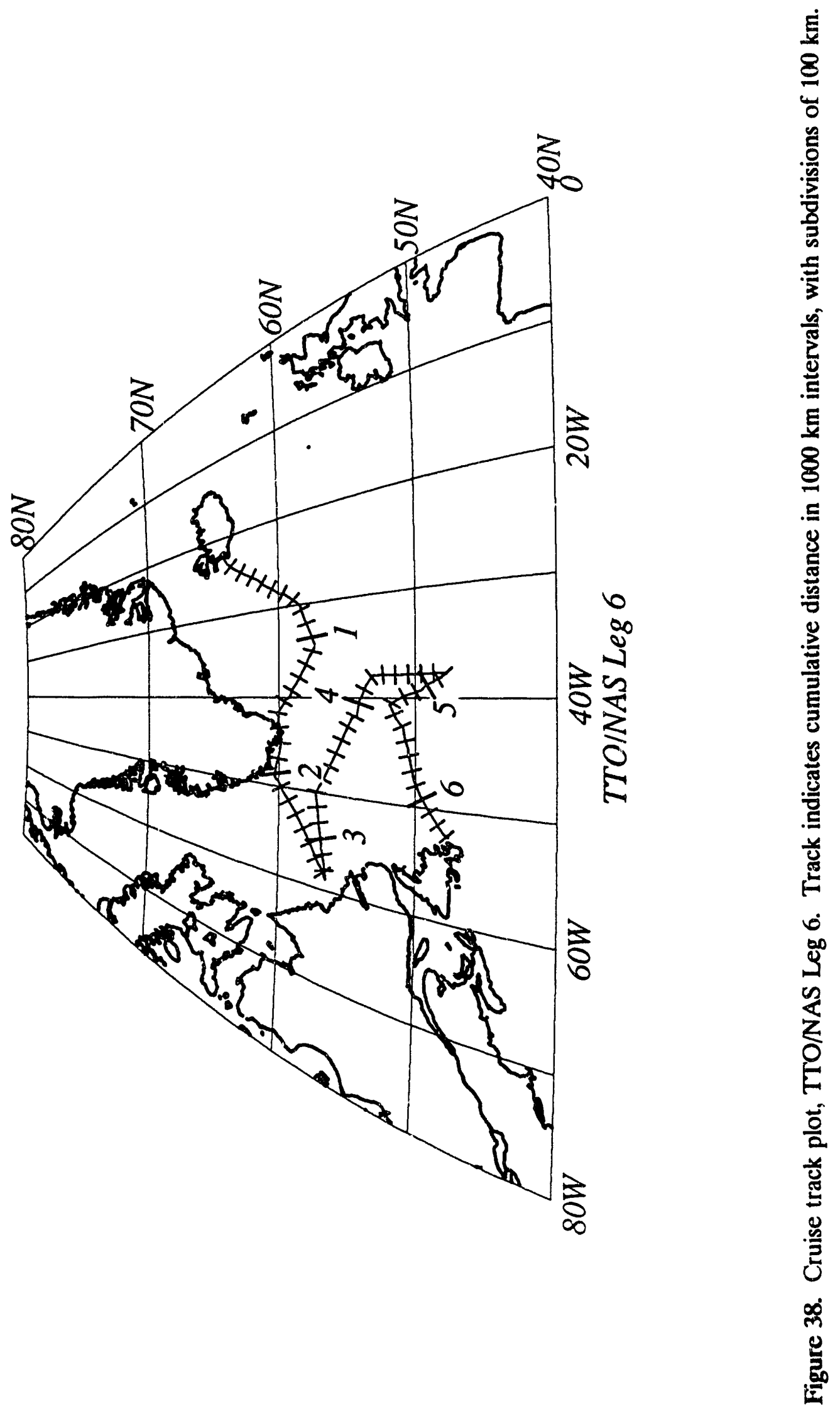




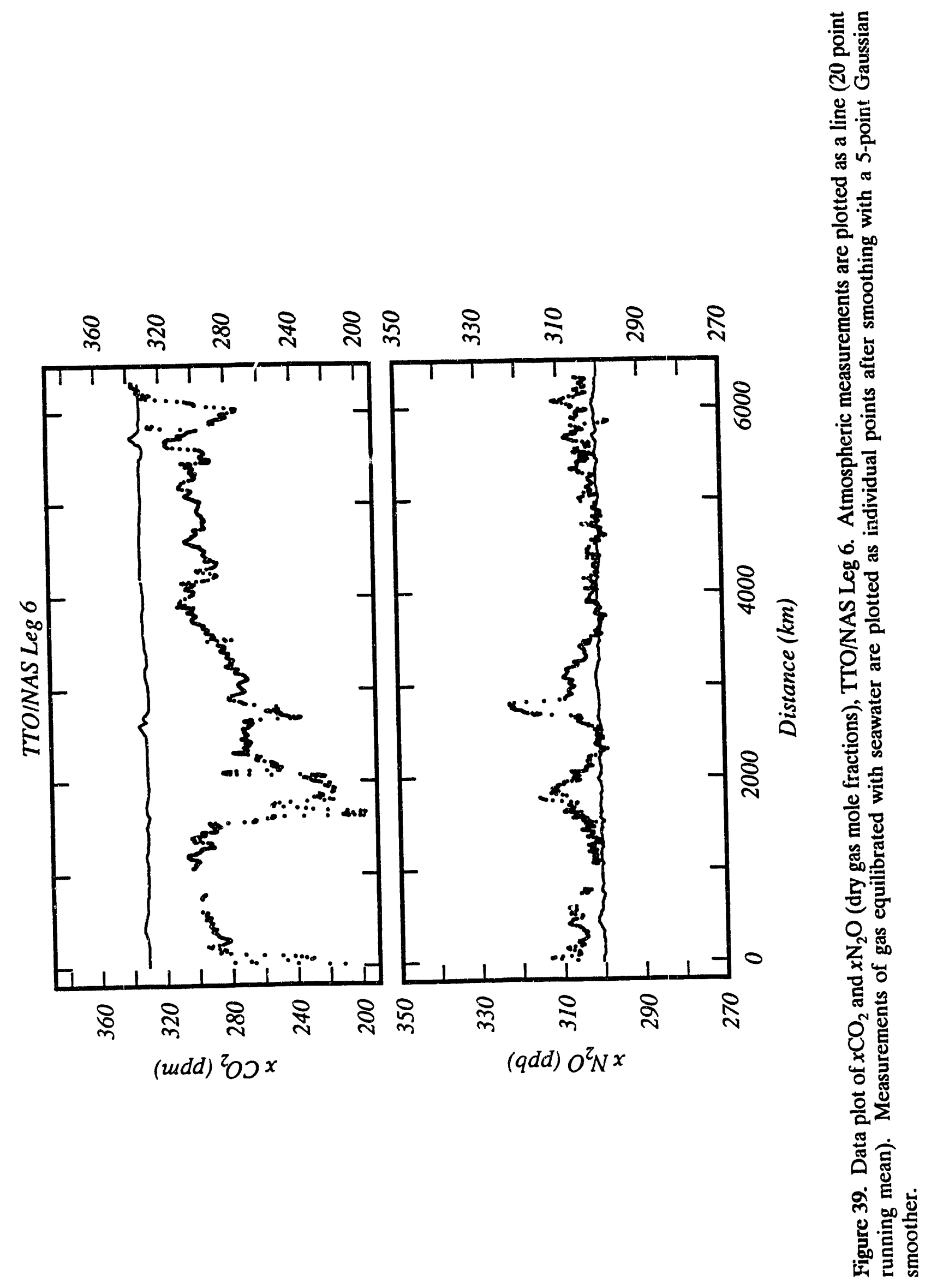




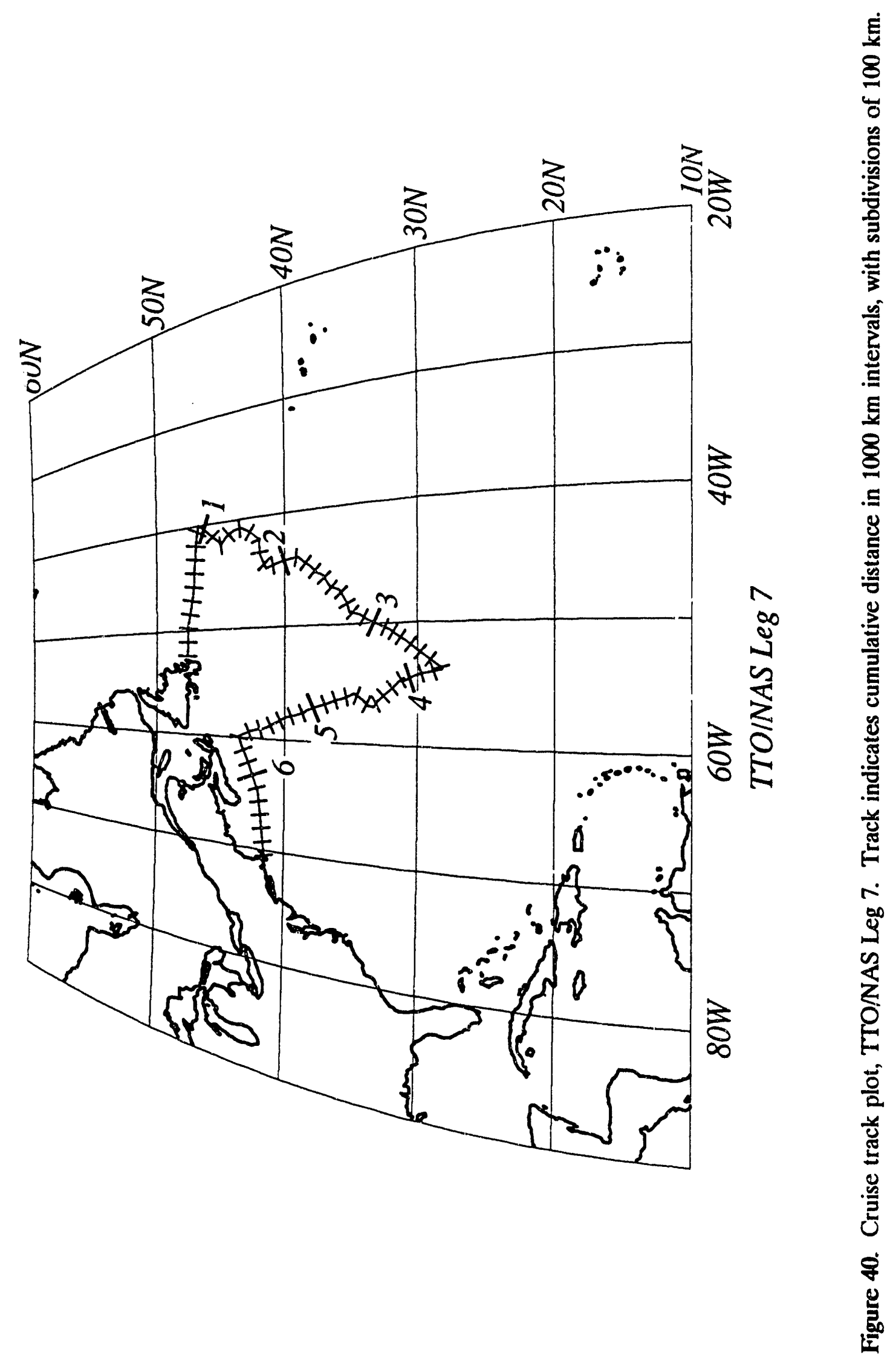




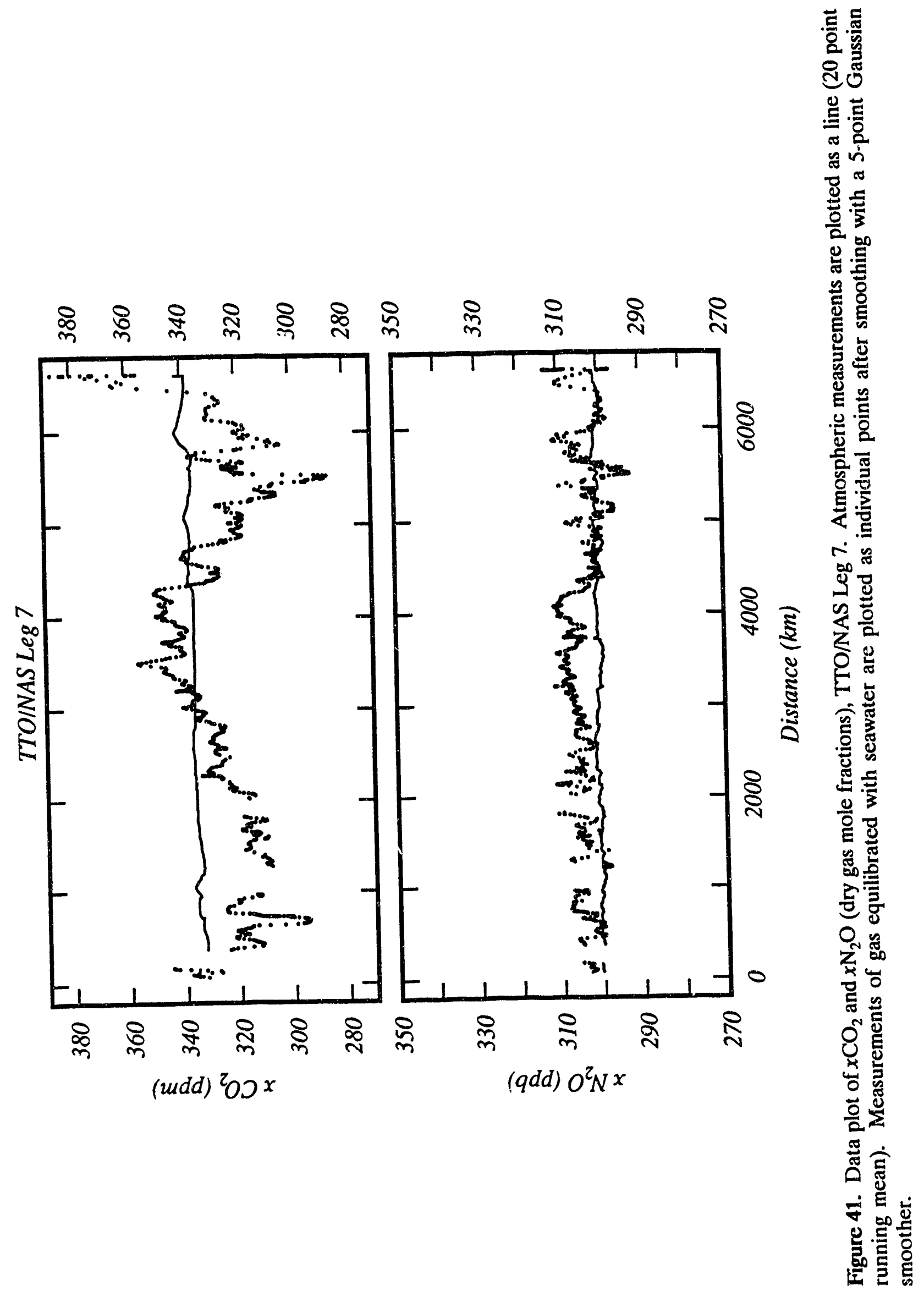




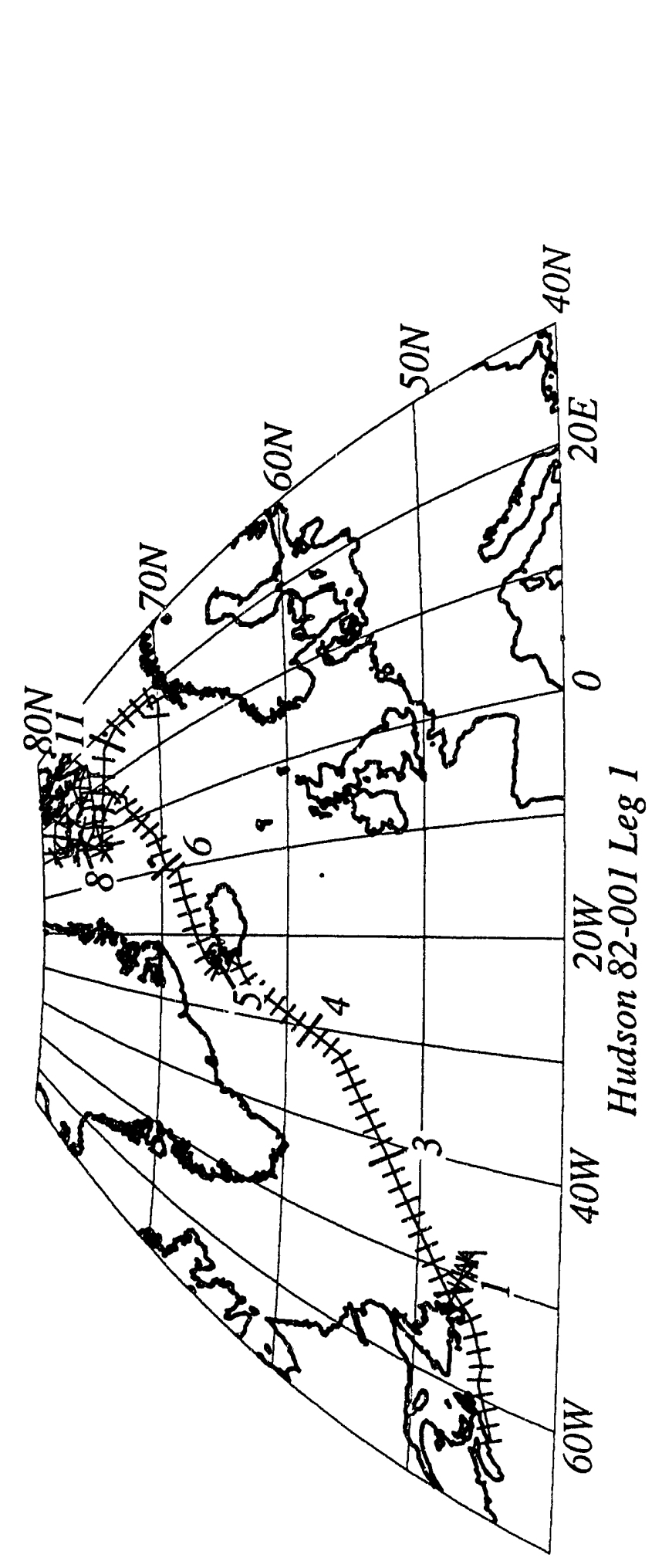

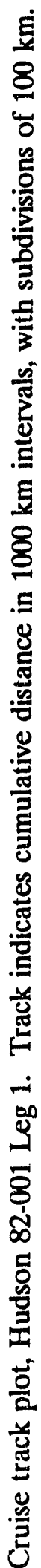

㞧 


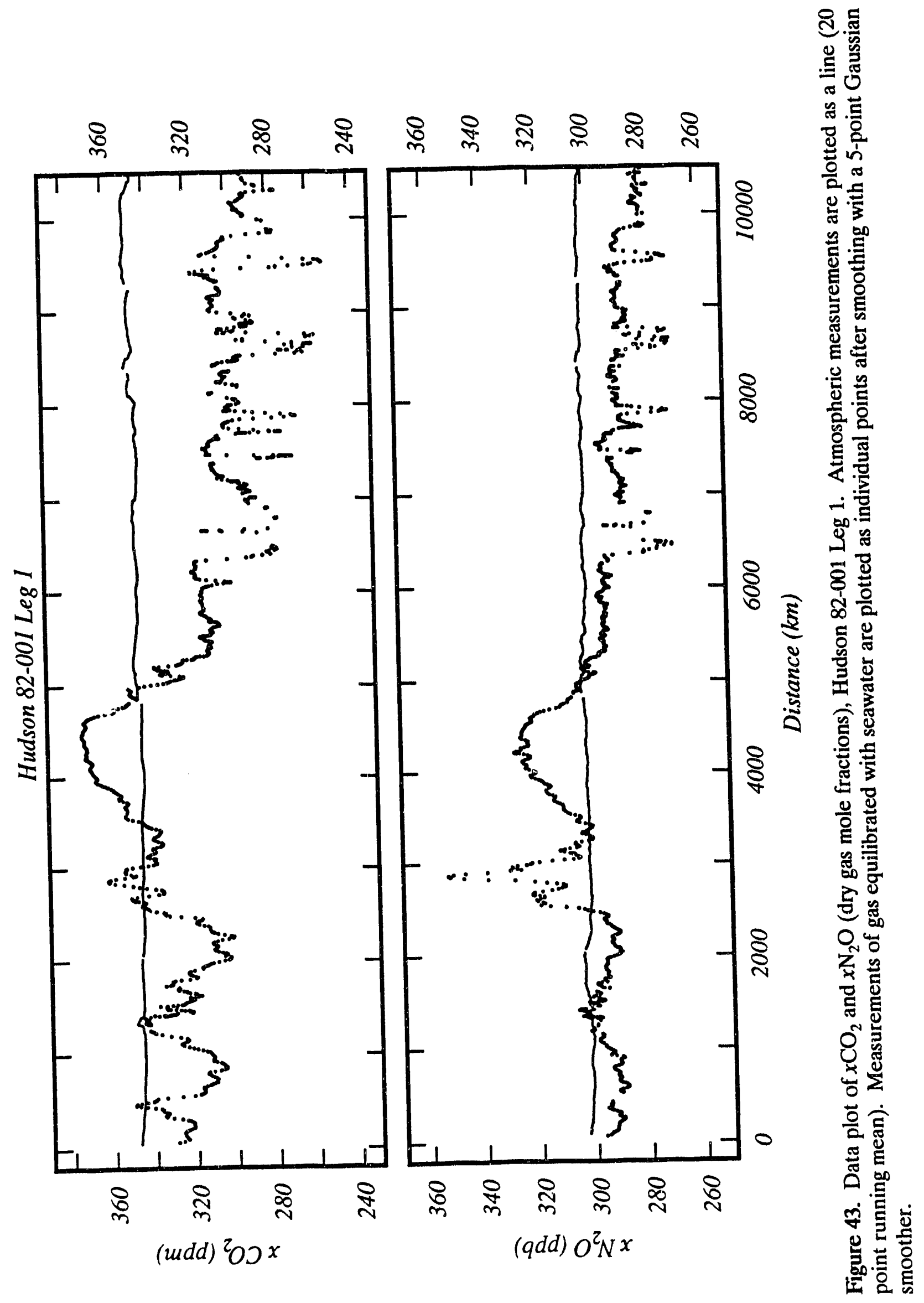




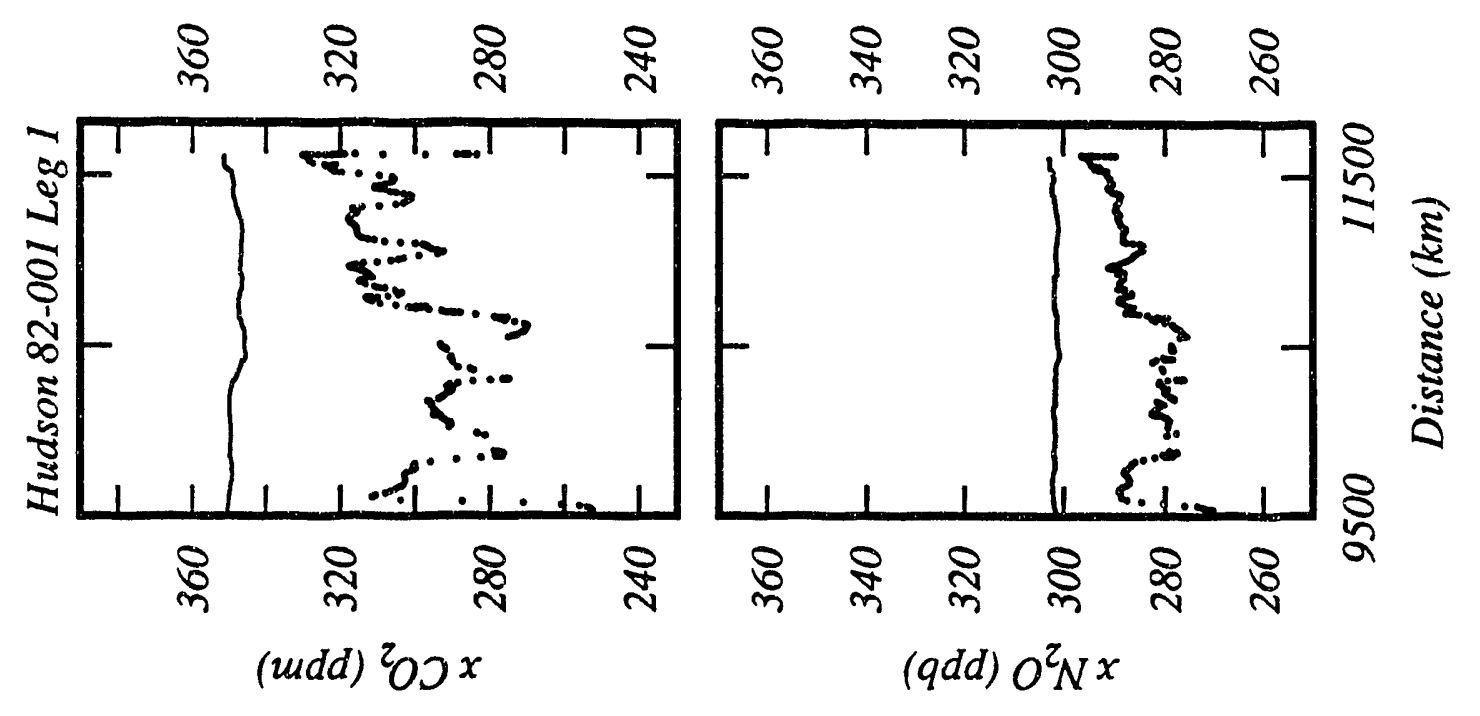

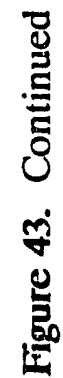




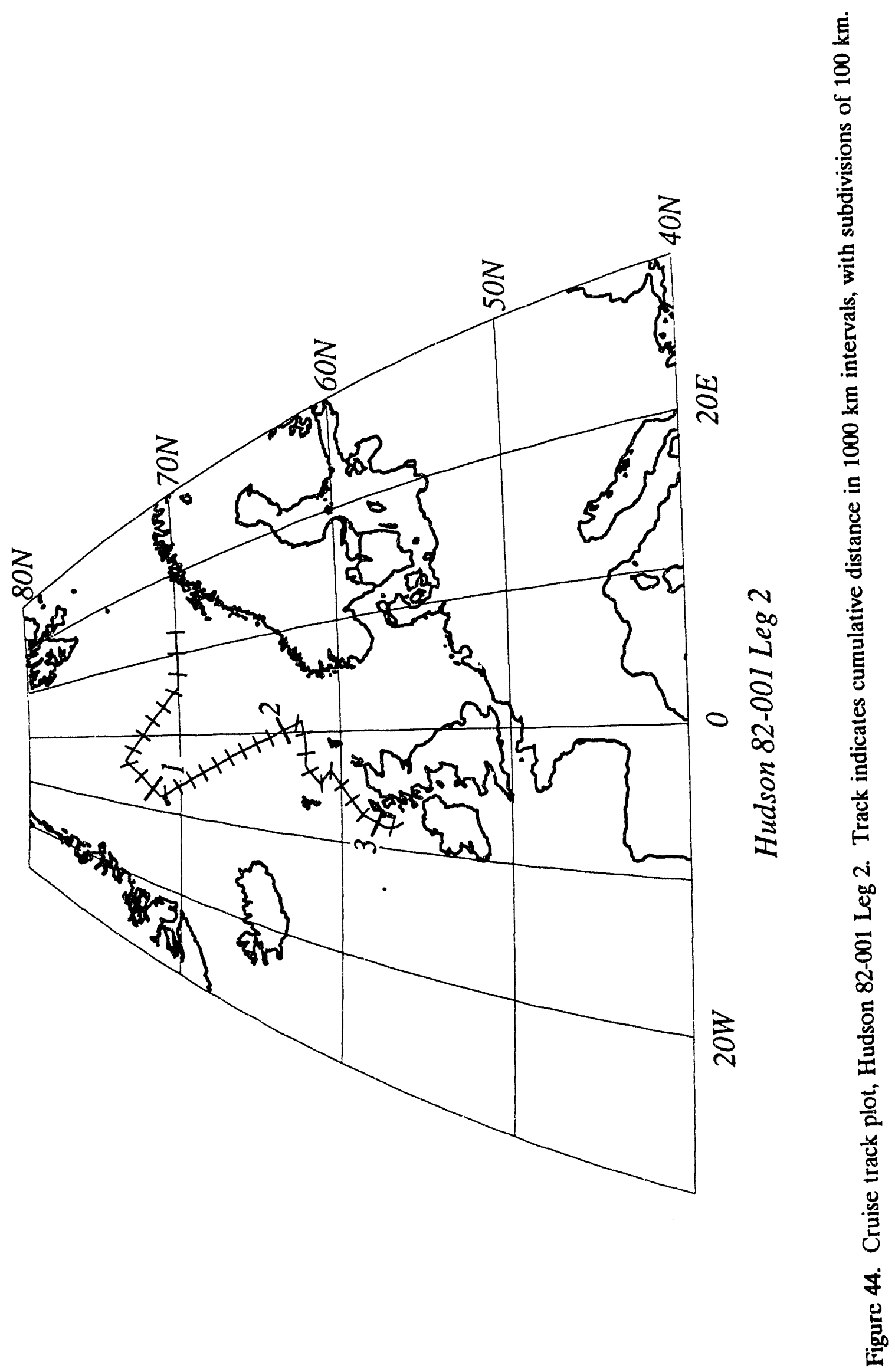




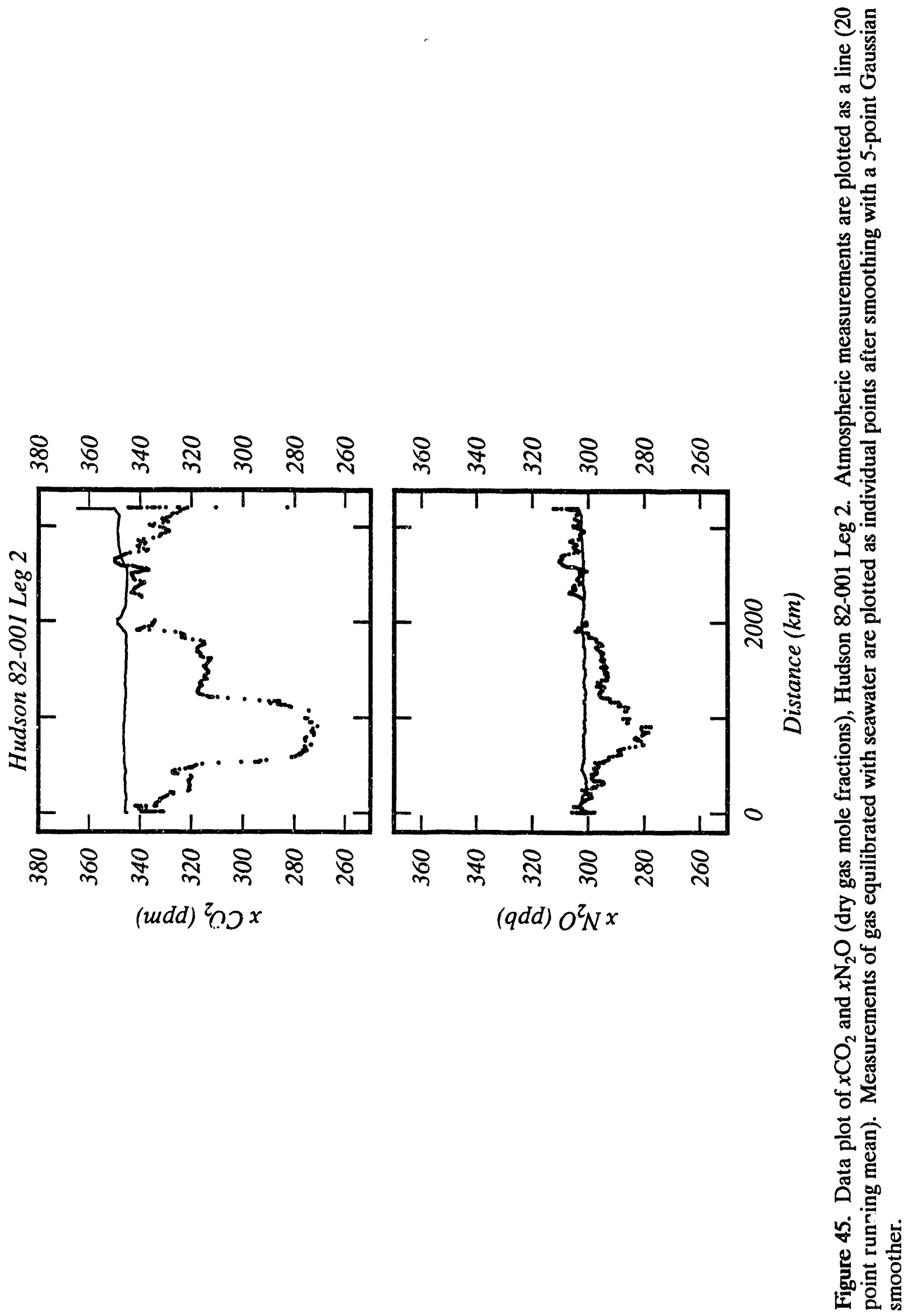




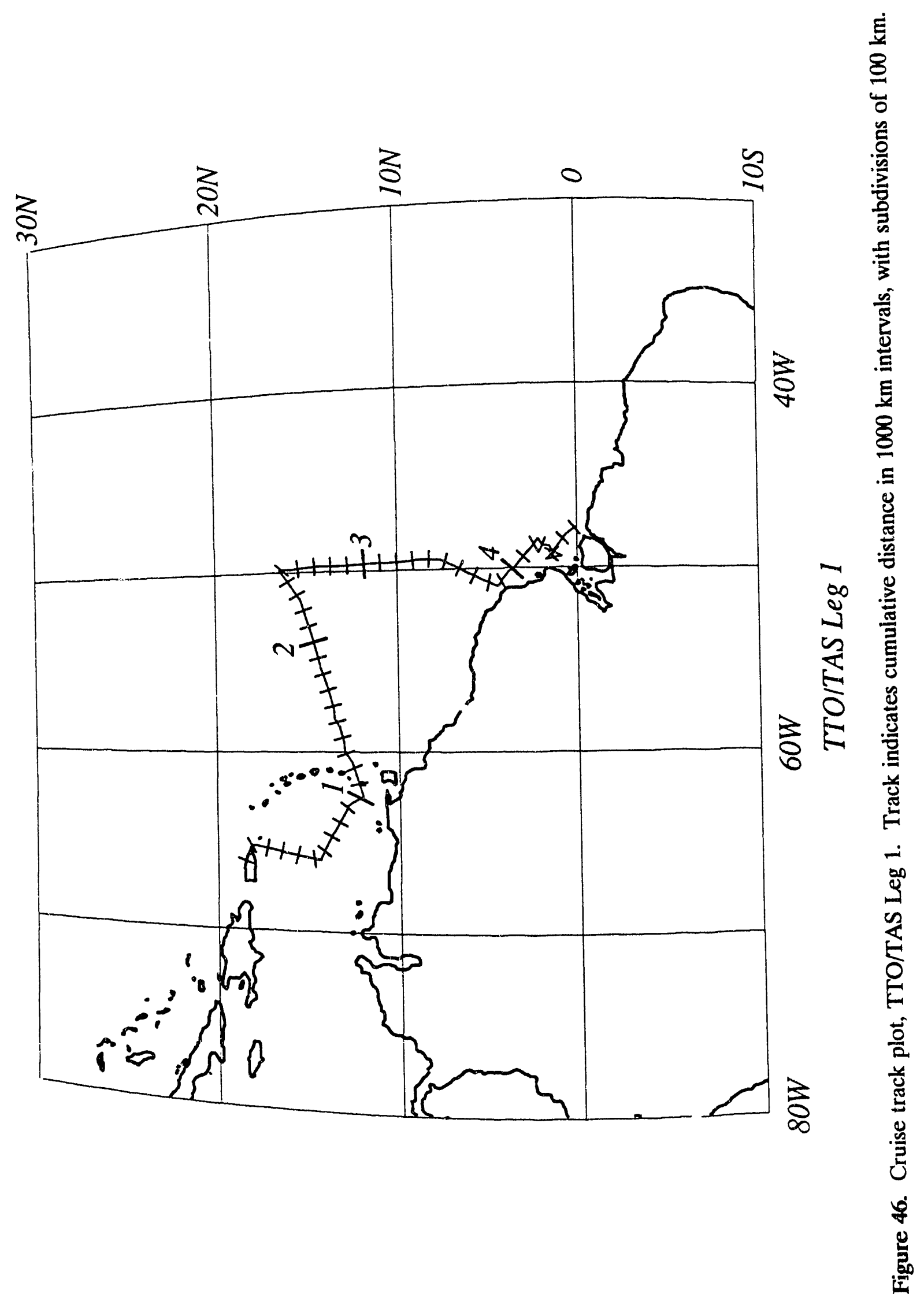




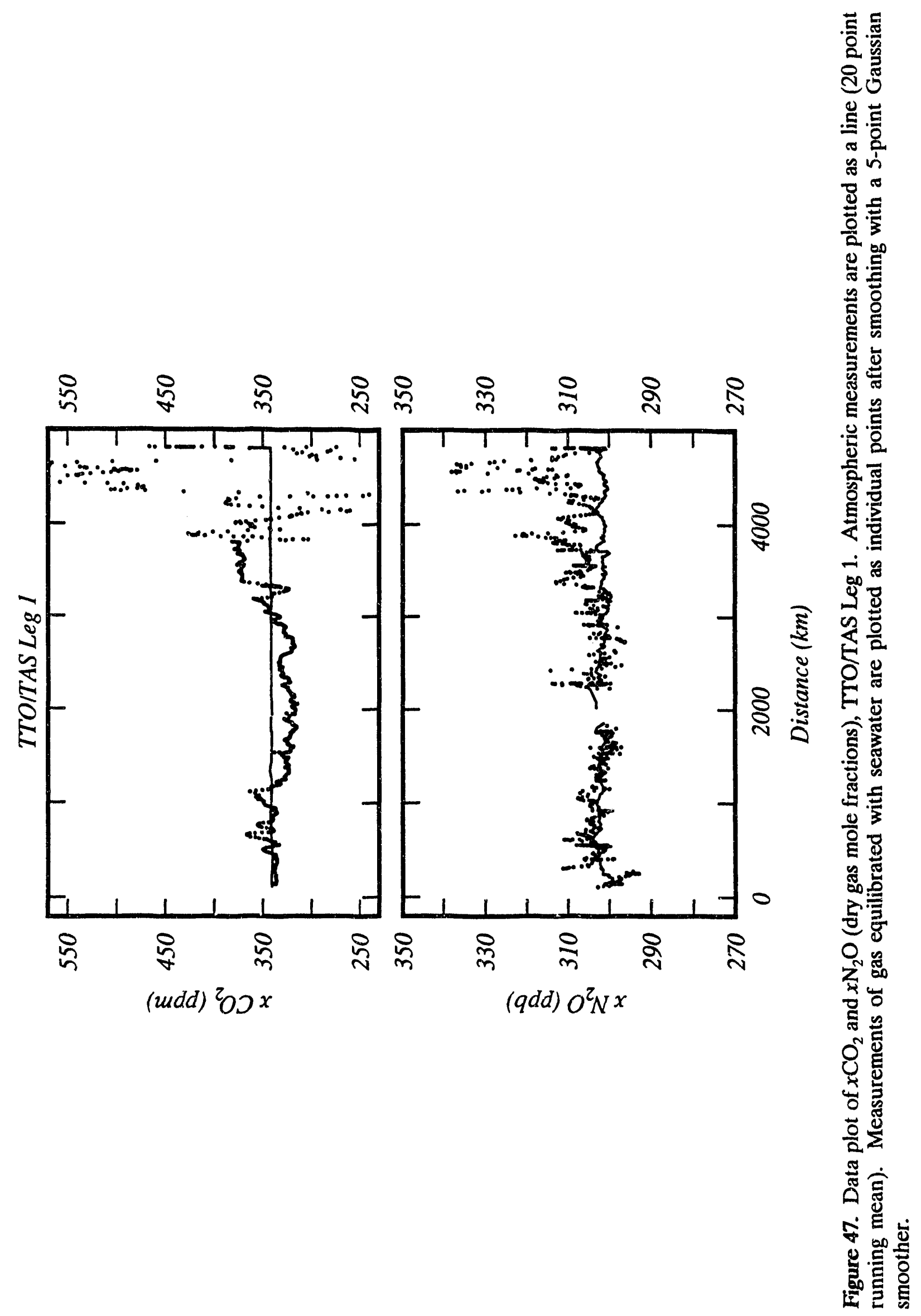




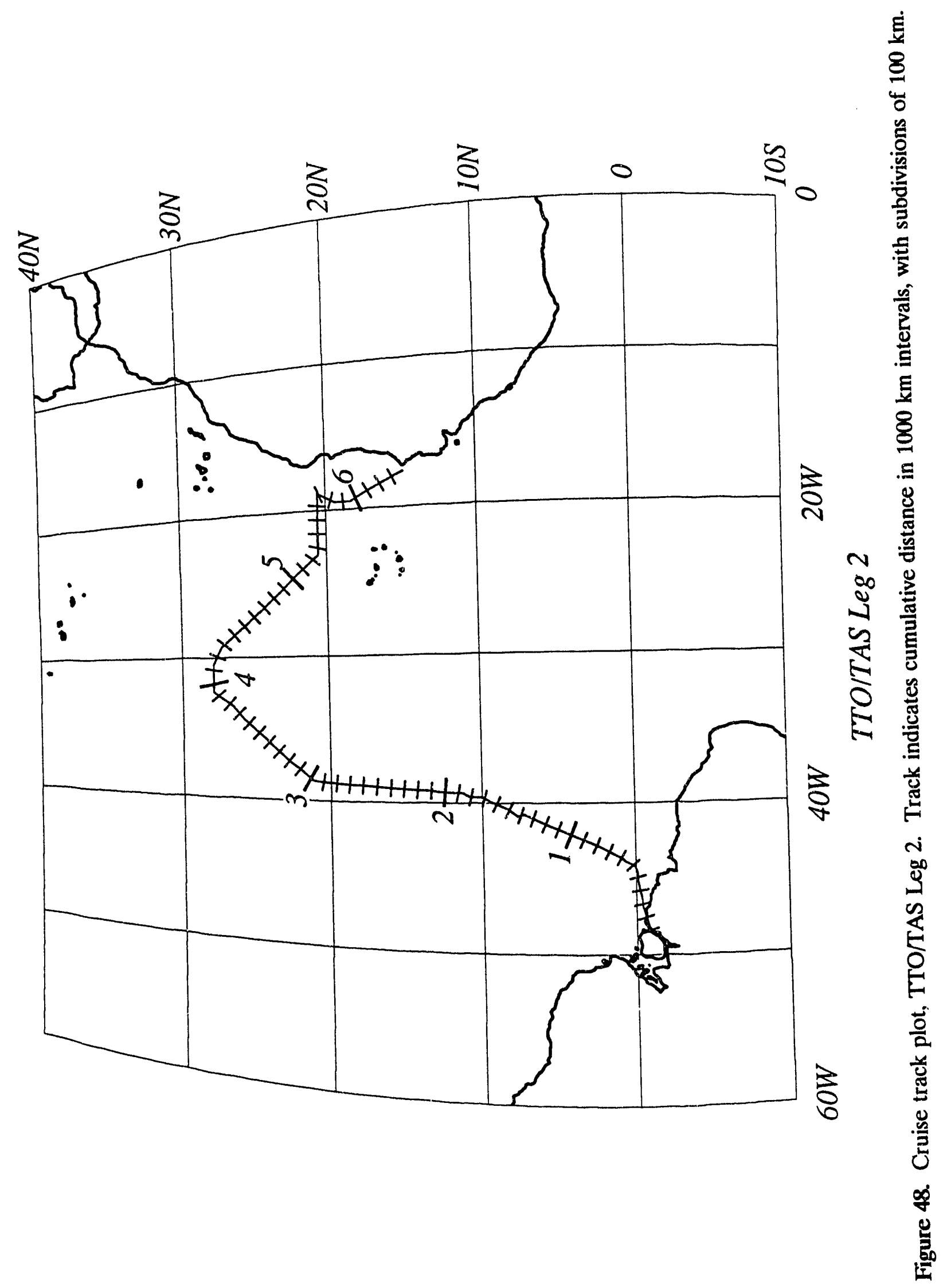




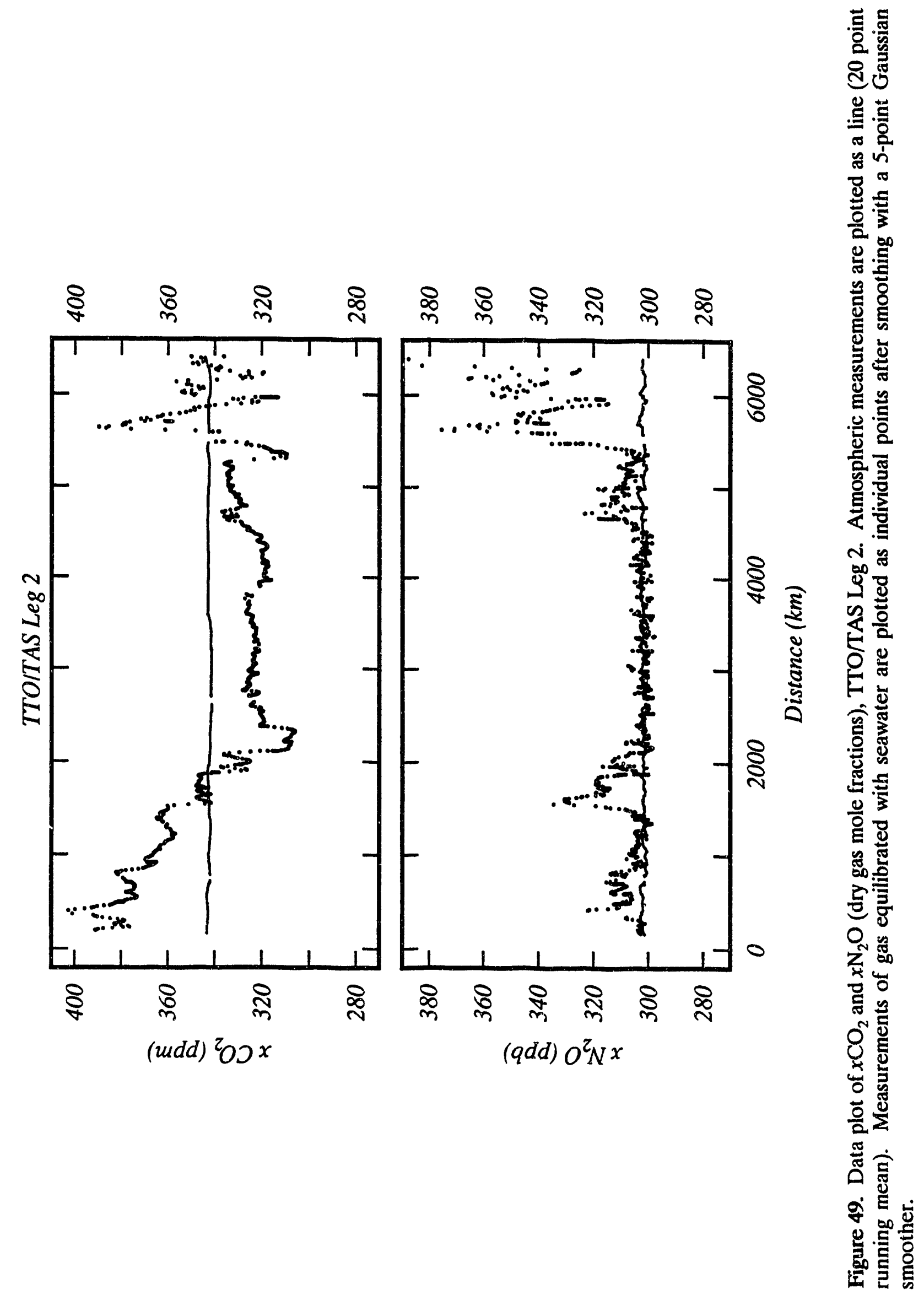




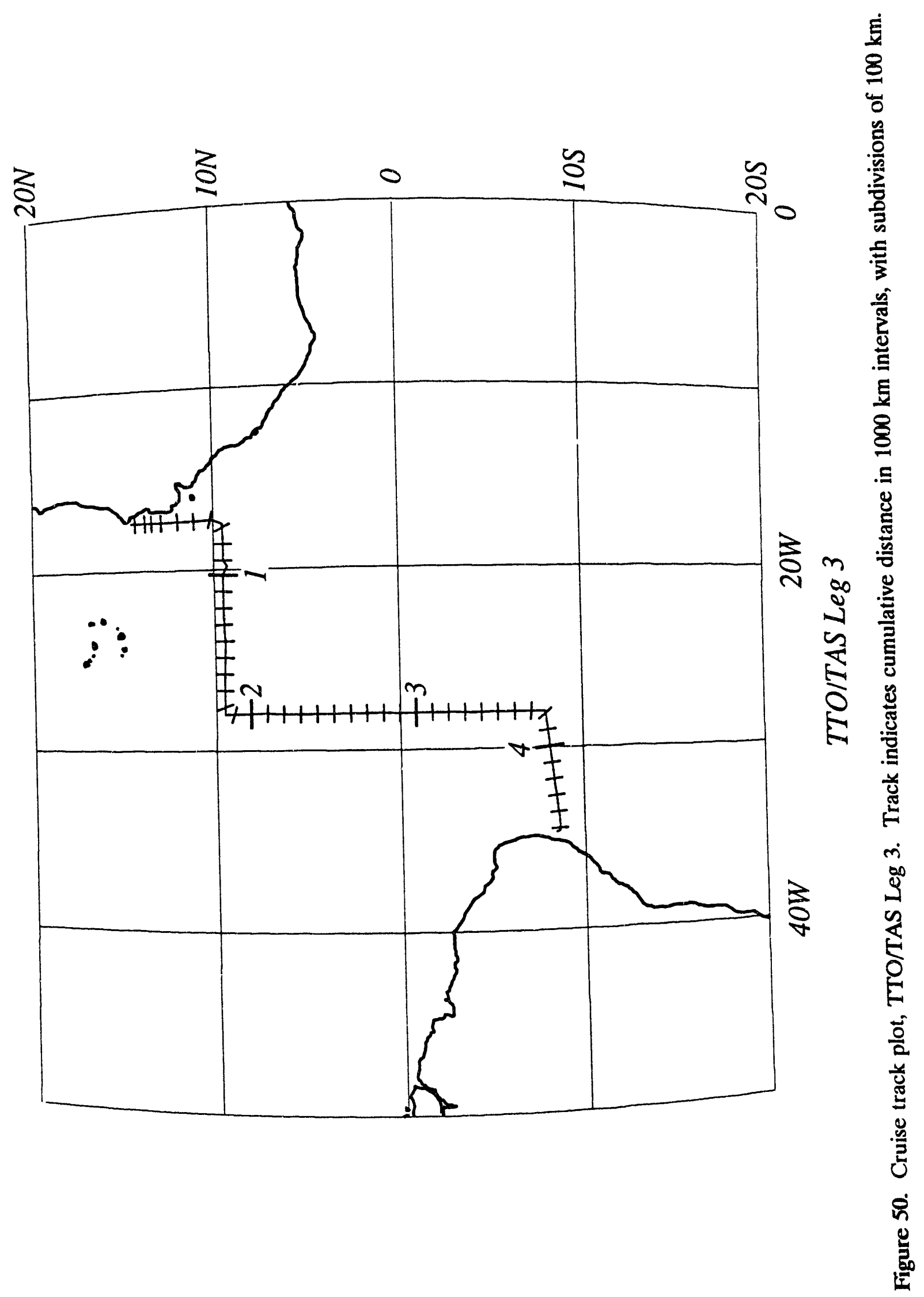




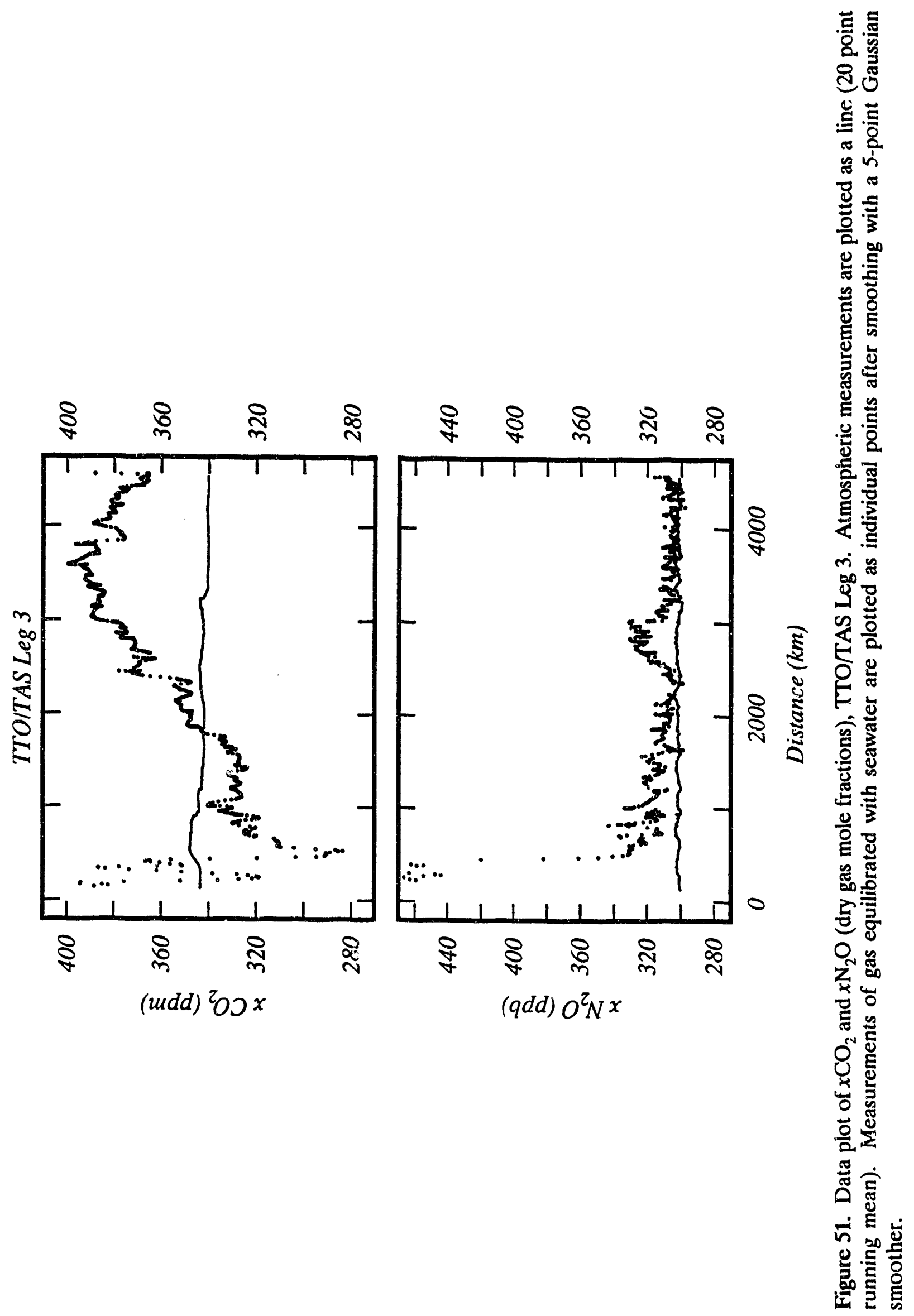



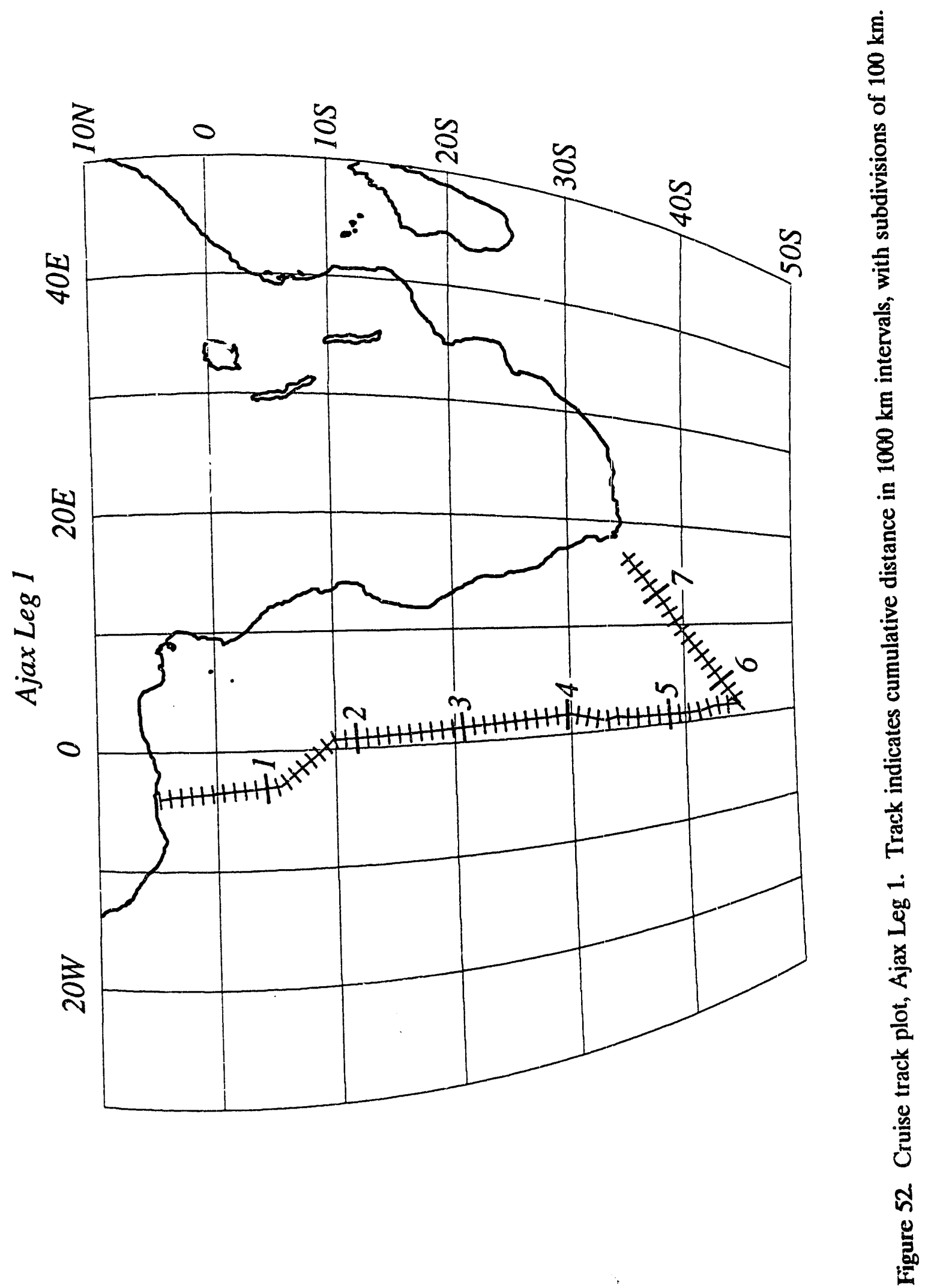


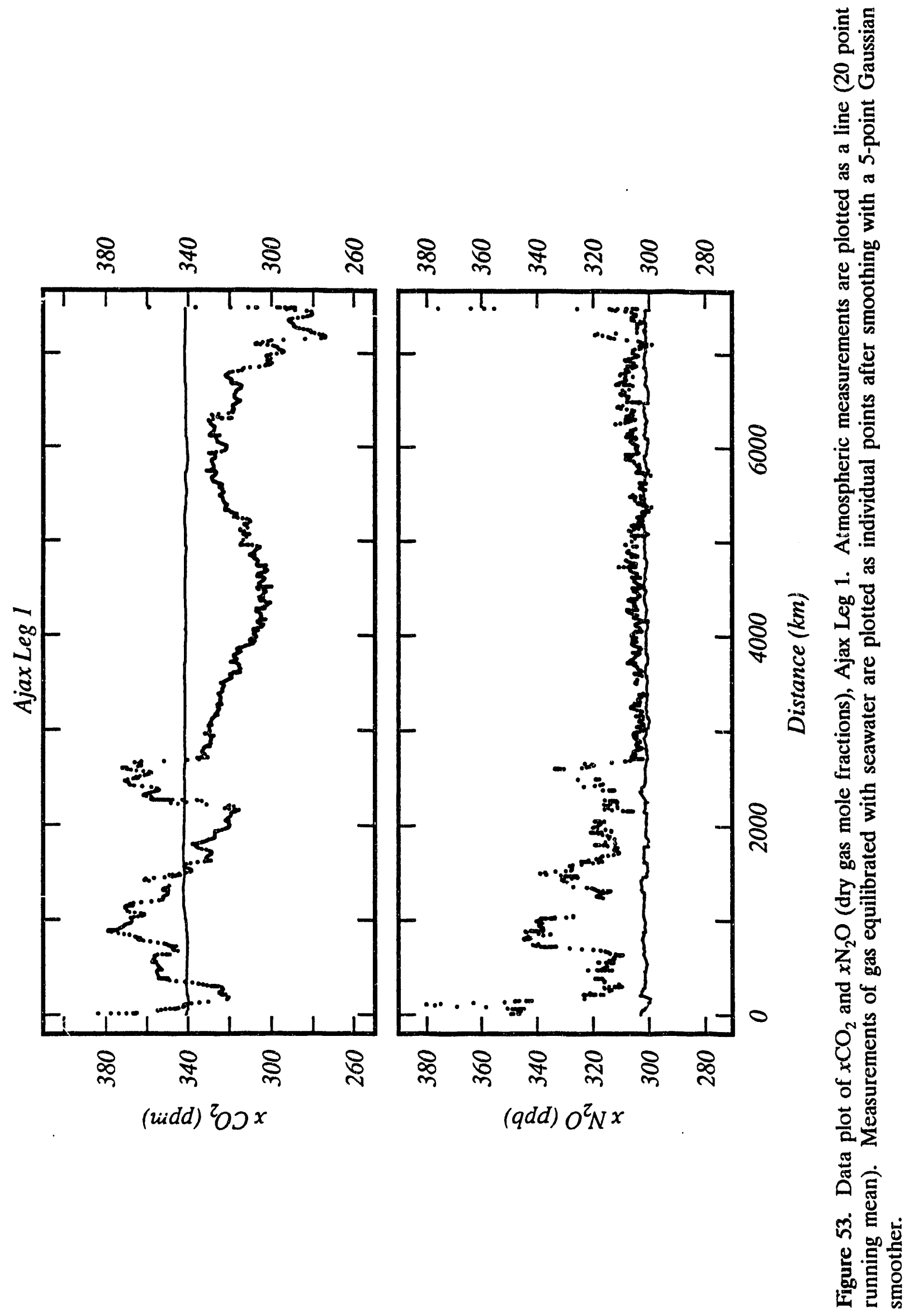




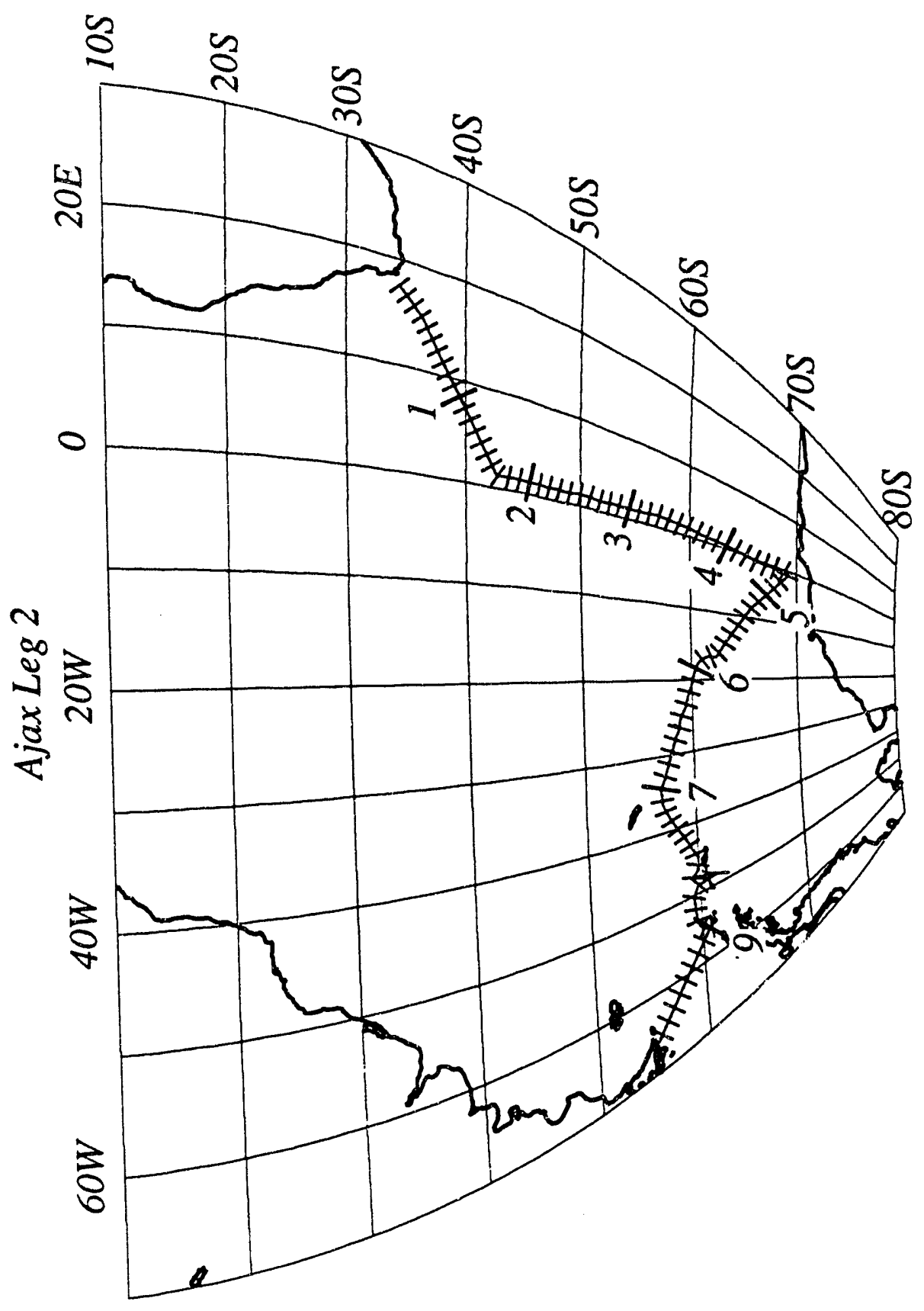

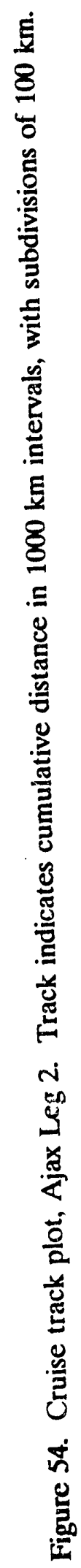




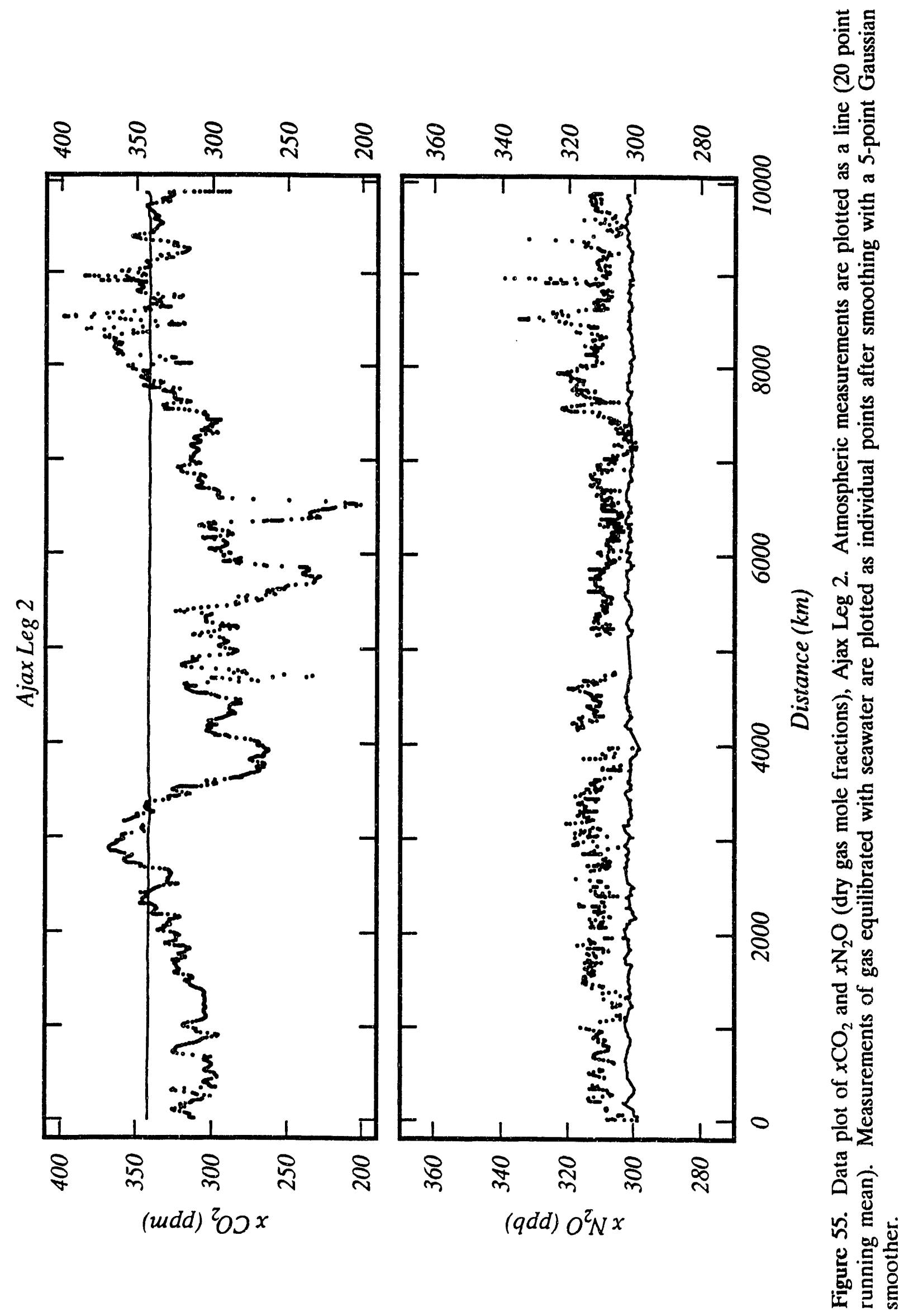




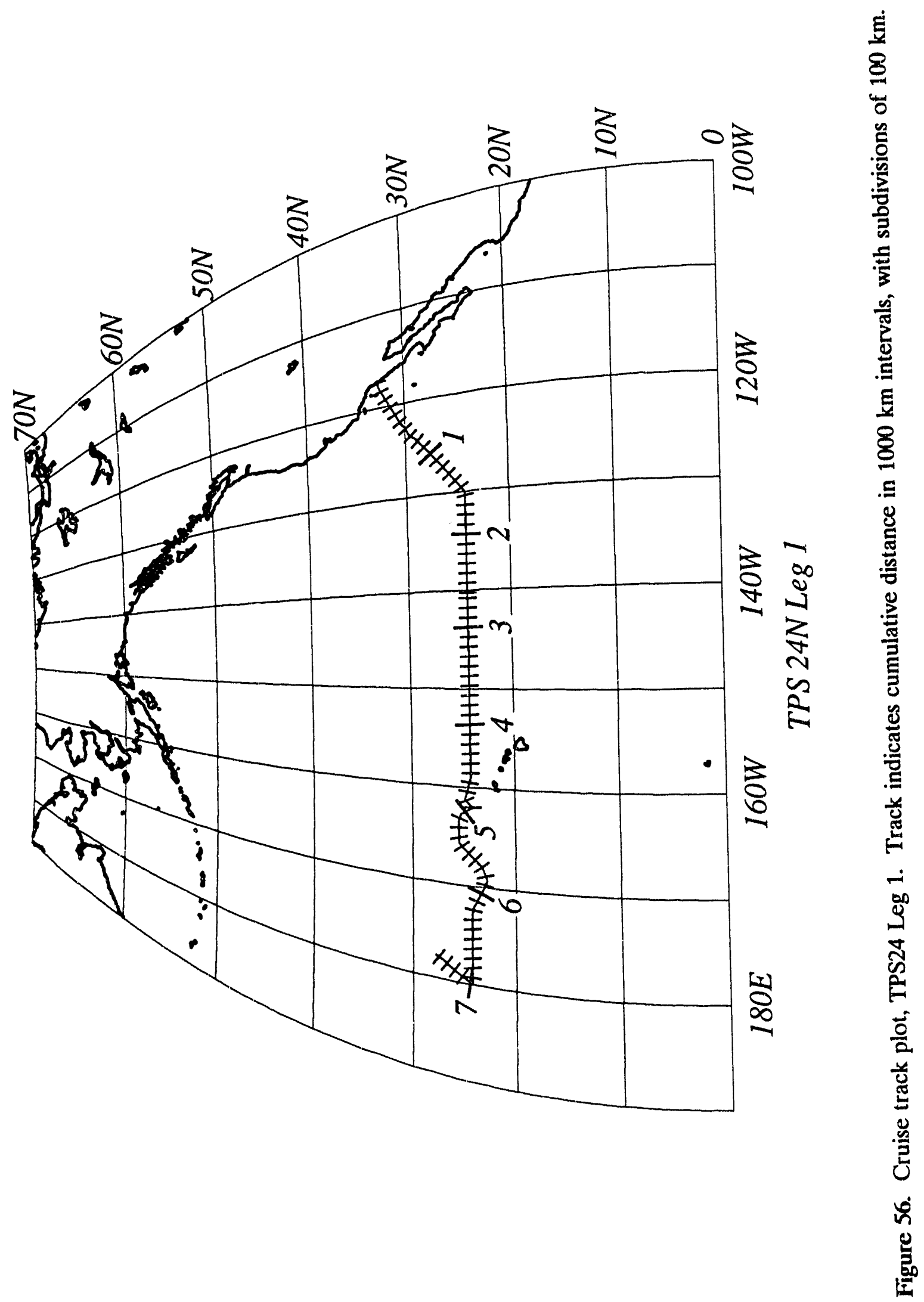




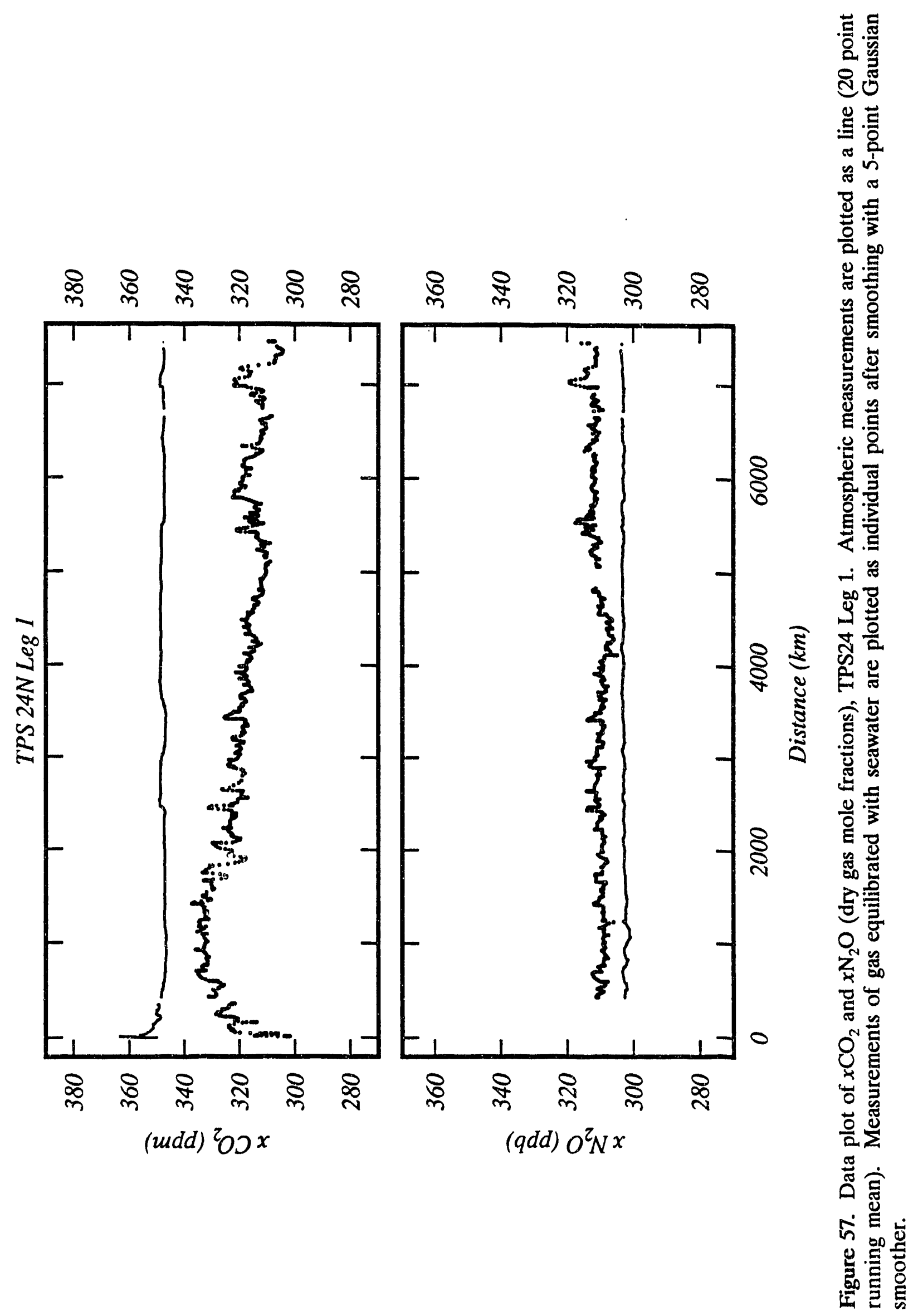




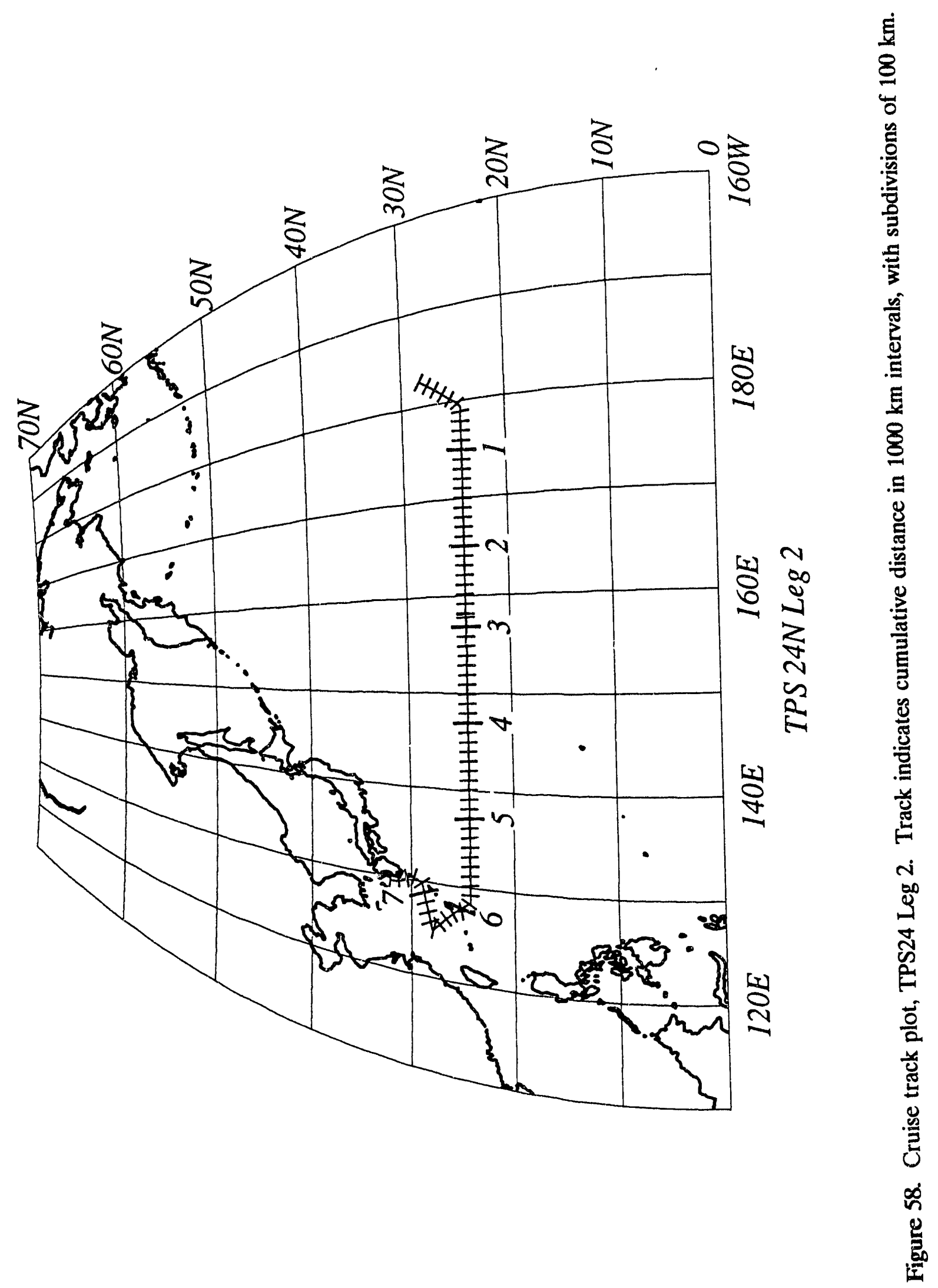




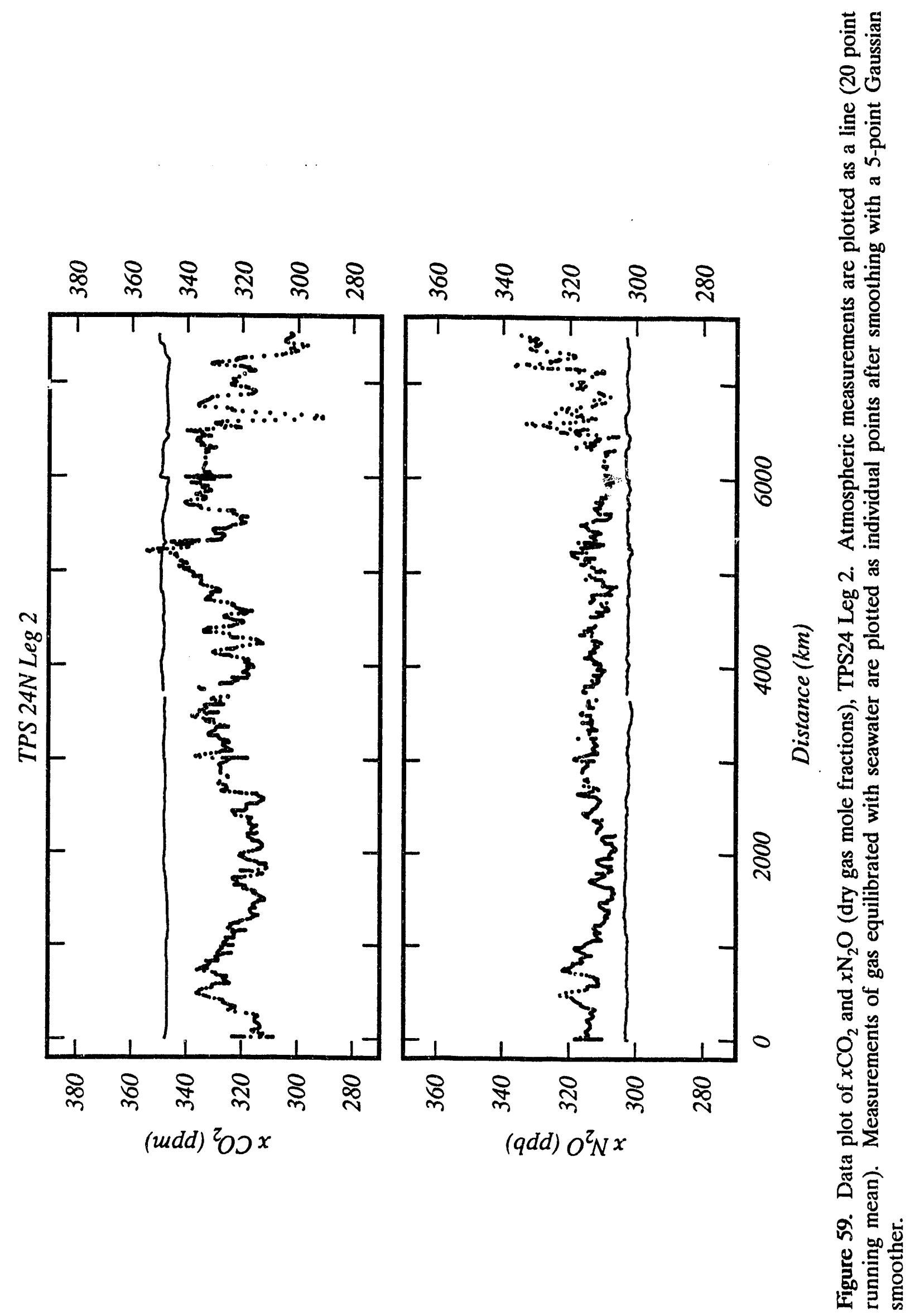




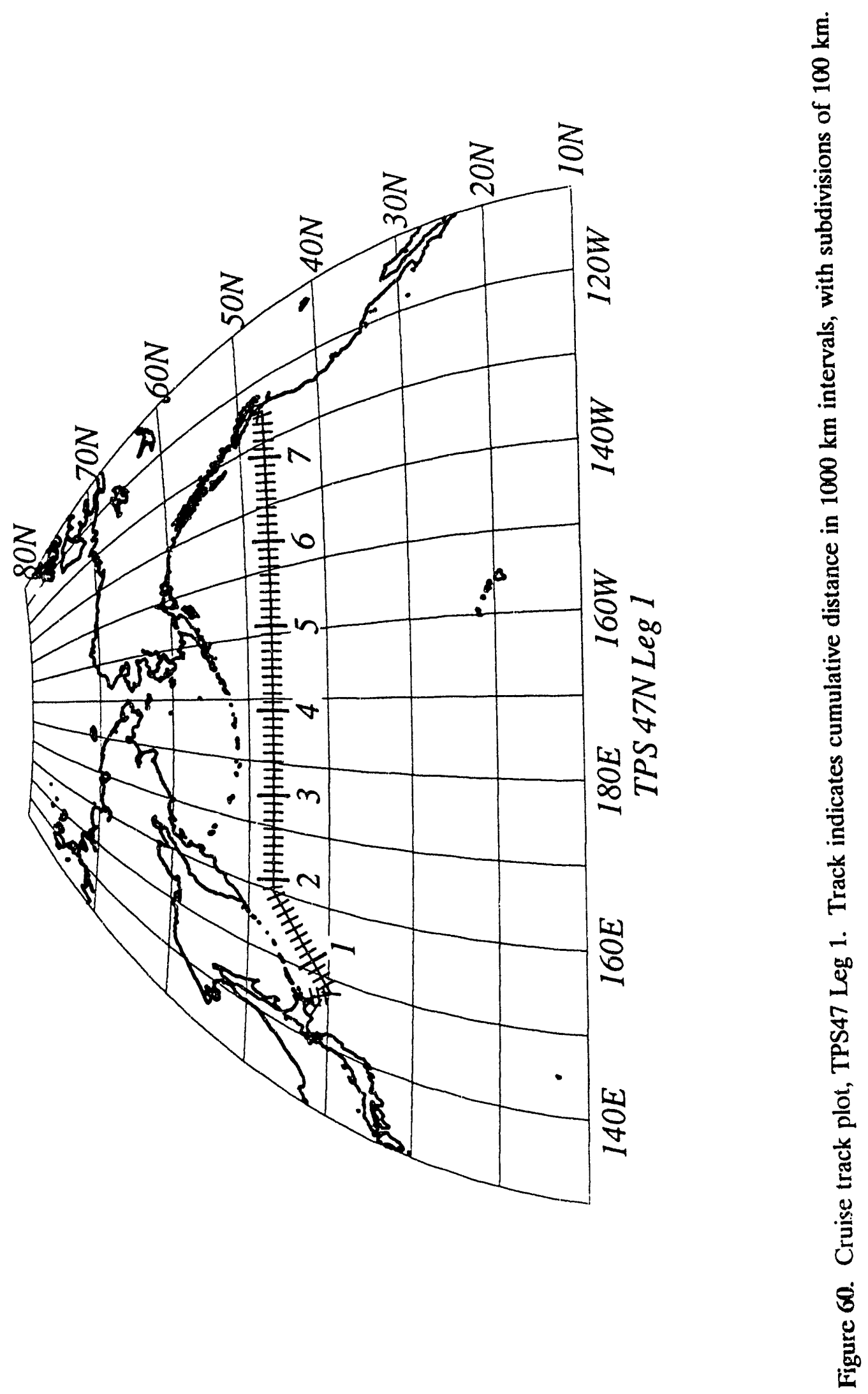




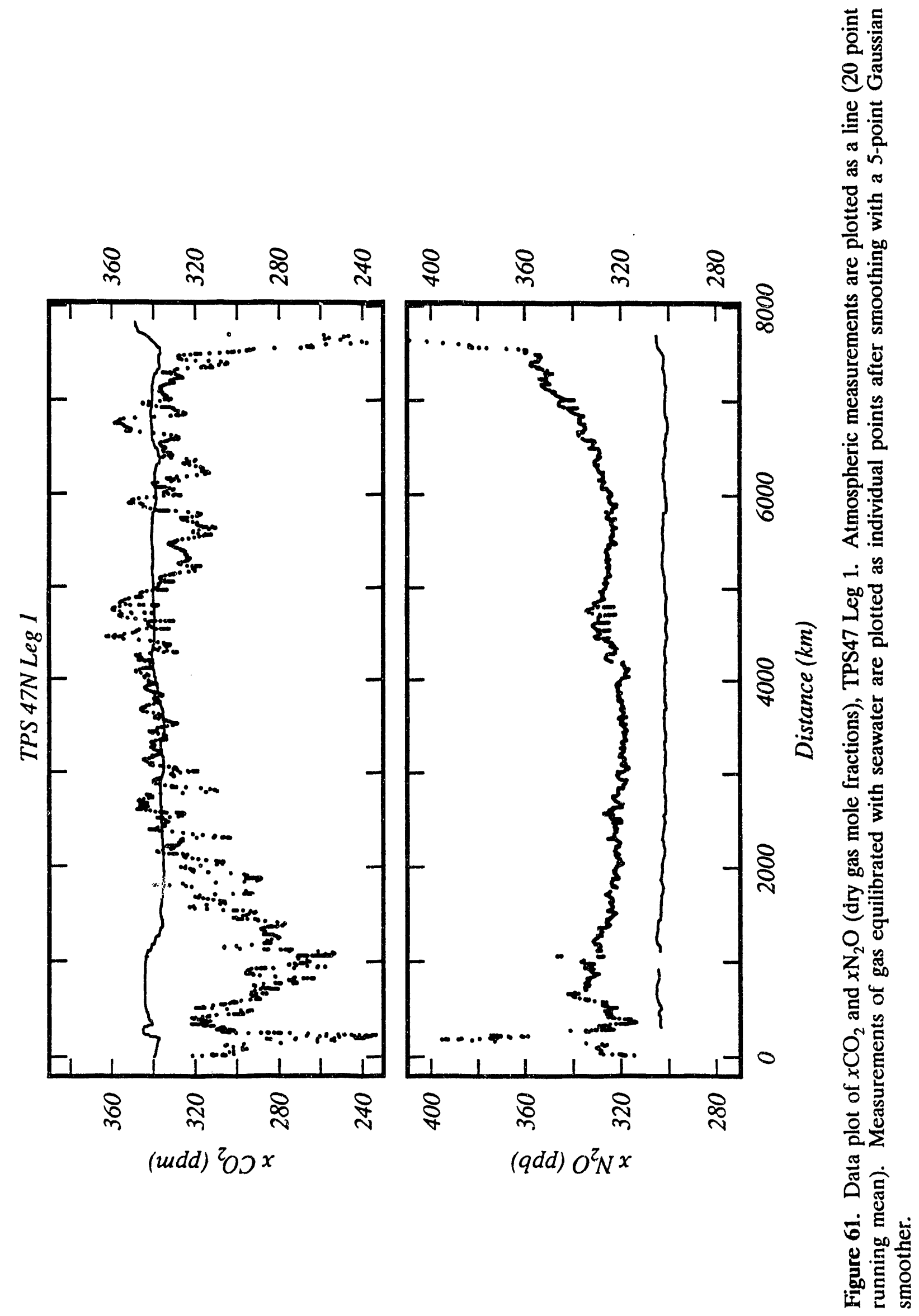



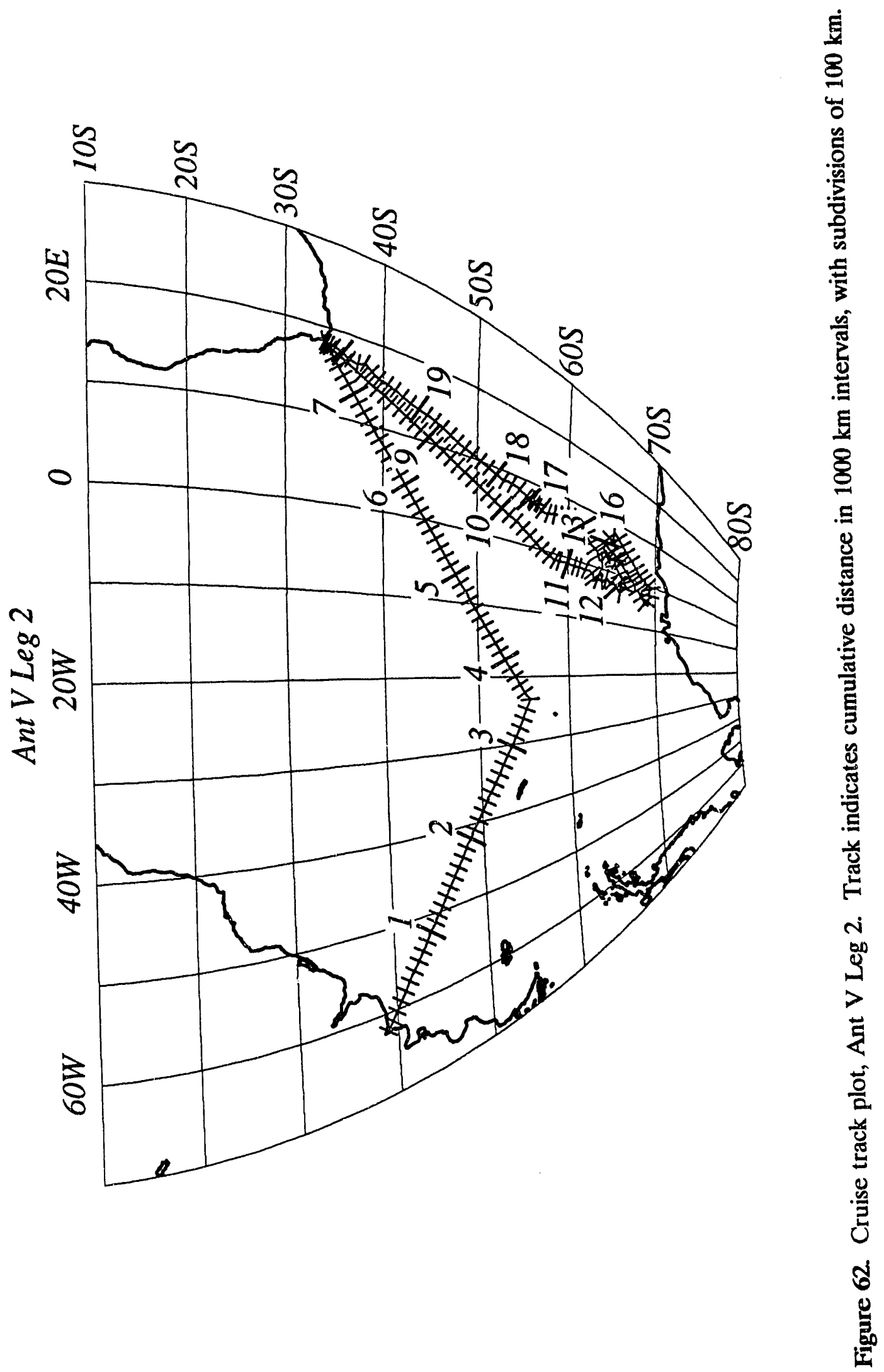


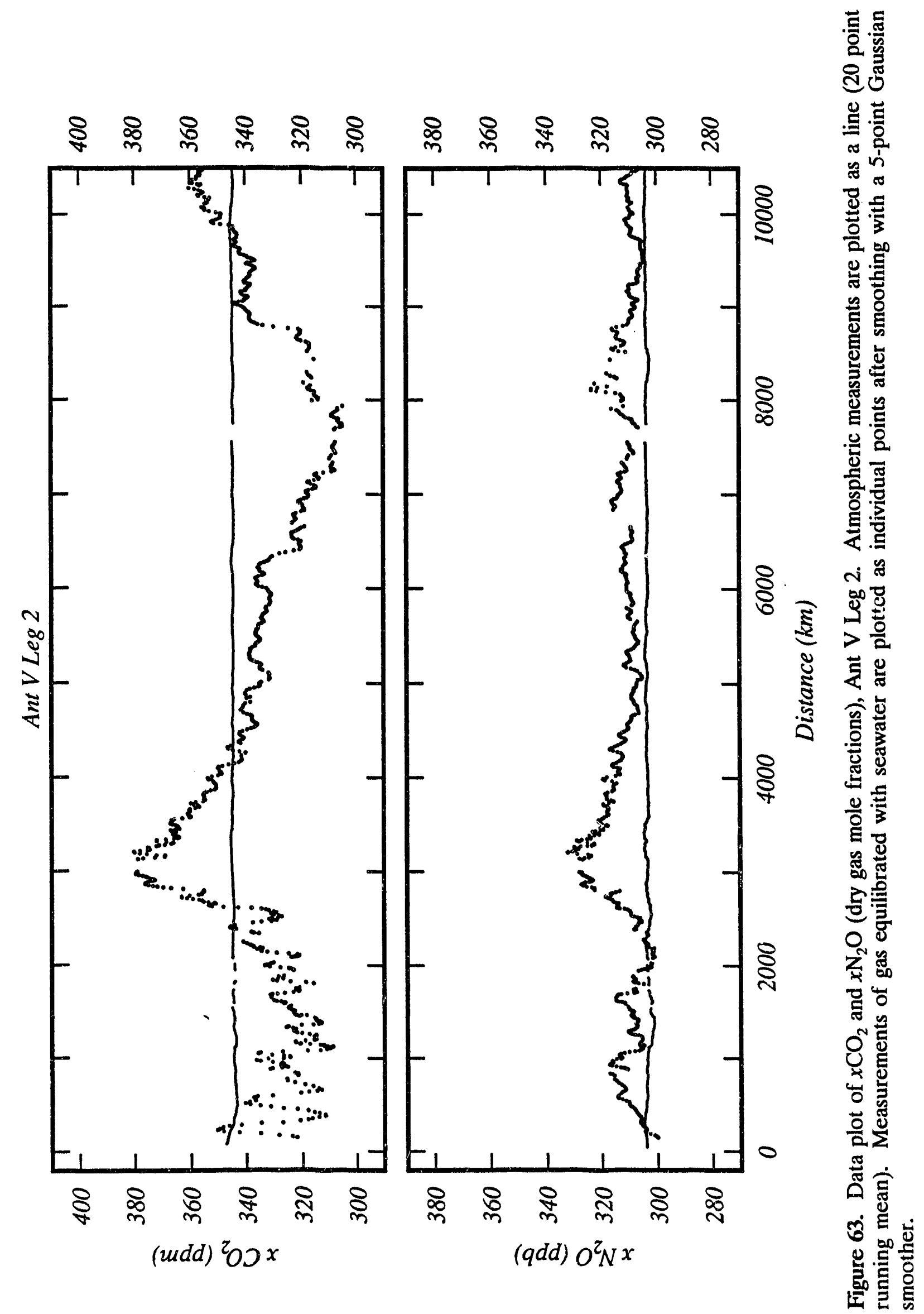




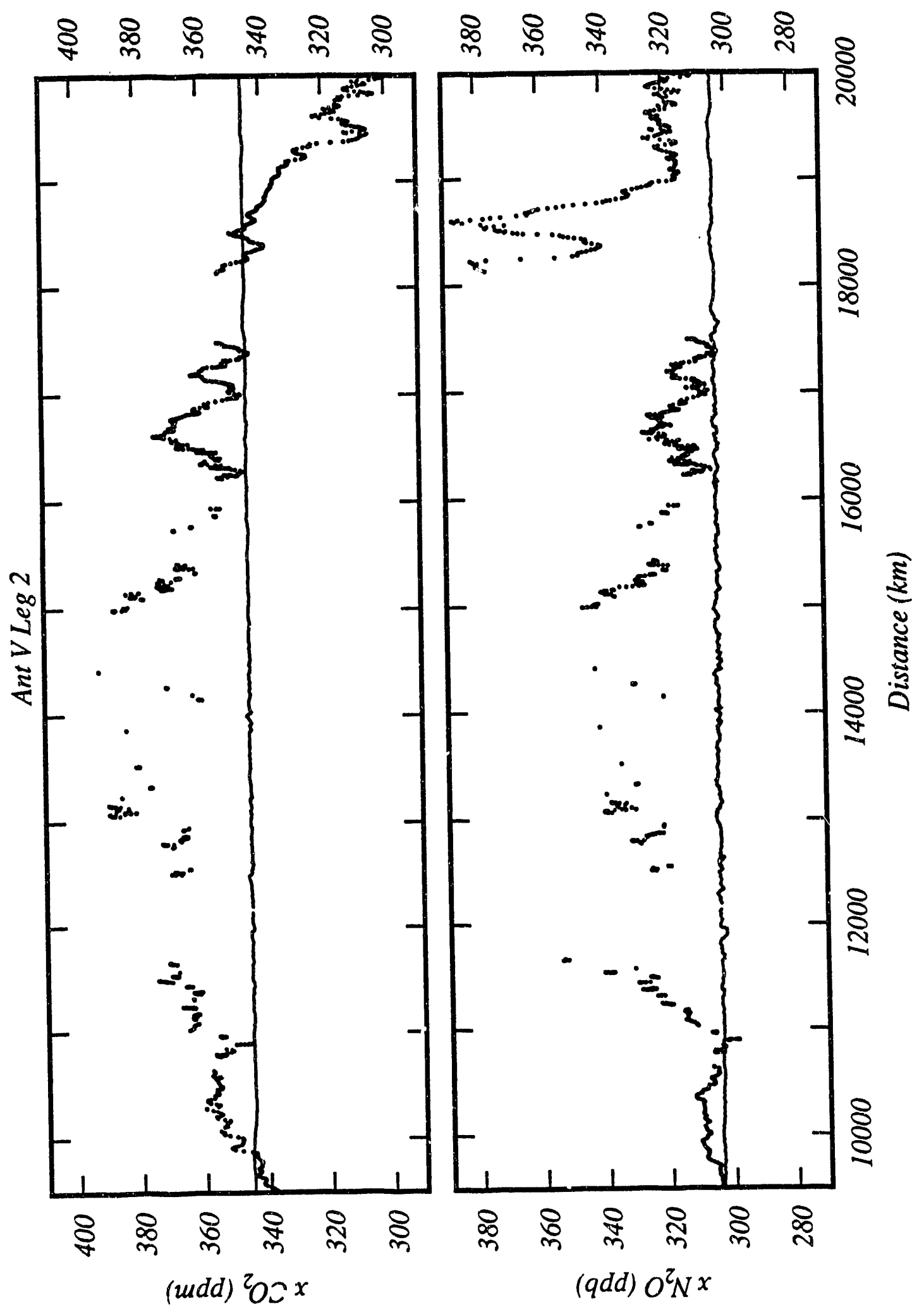

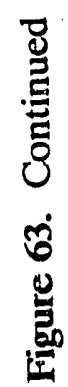




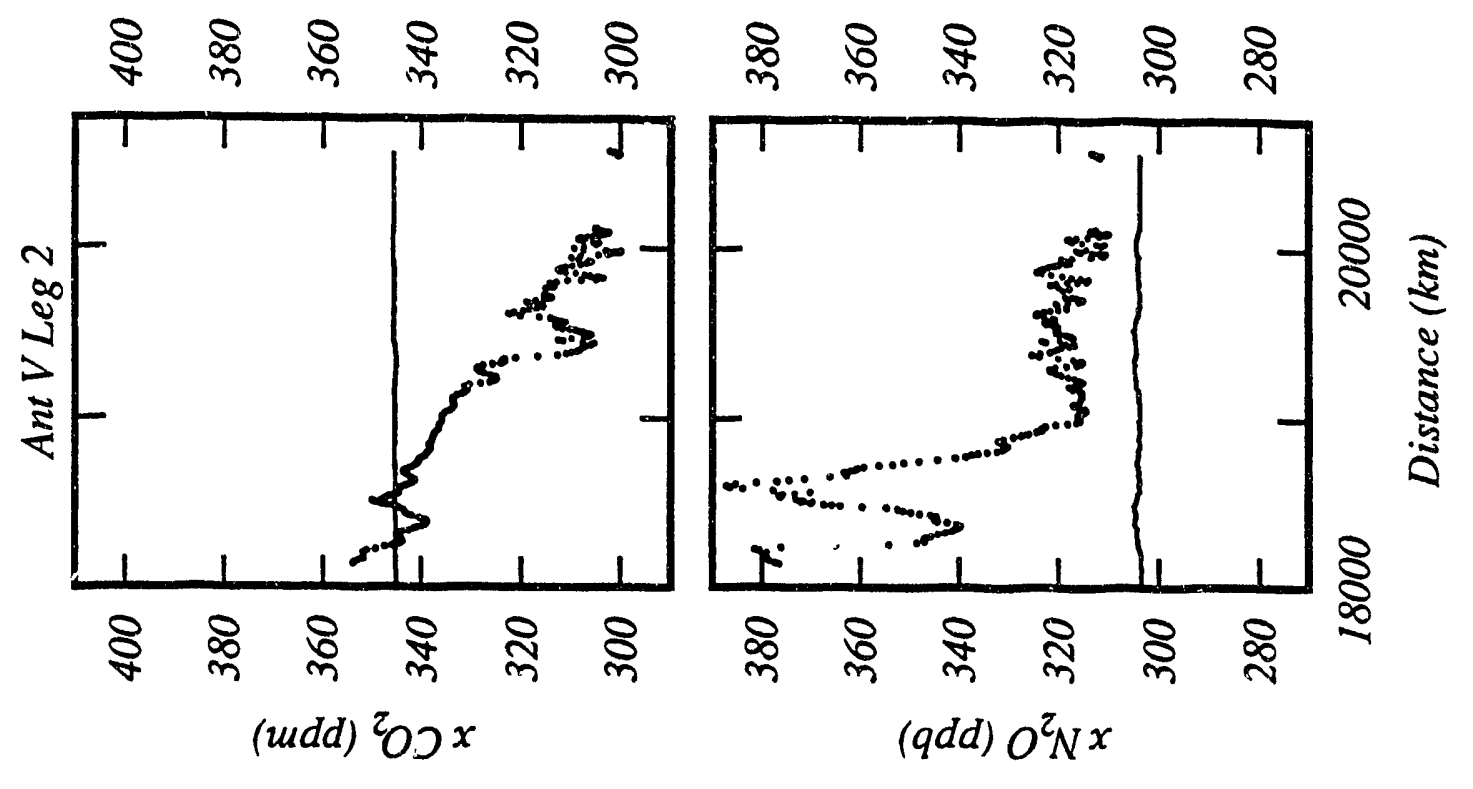




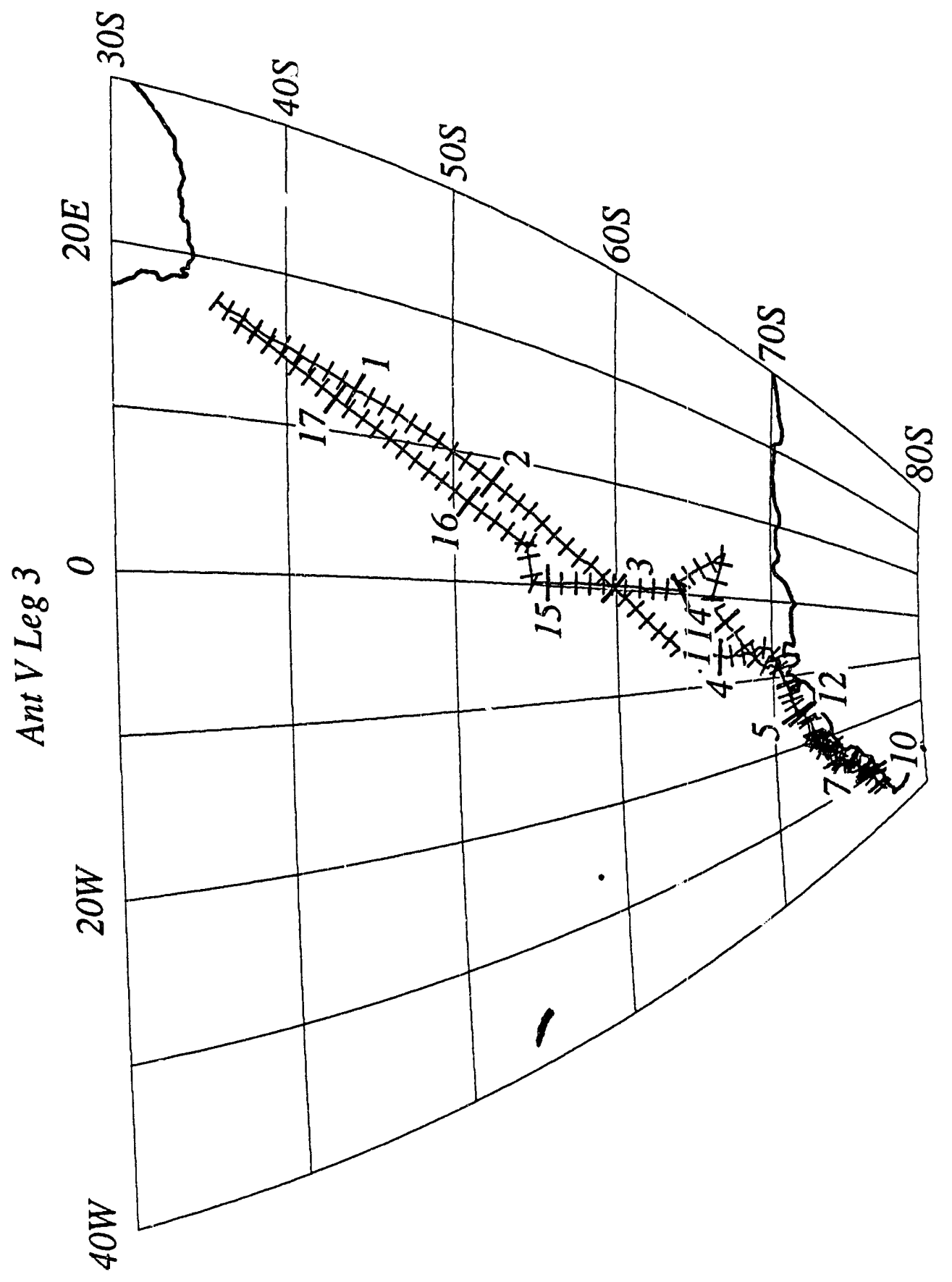

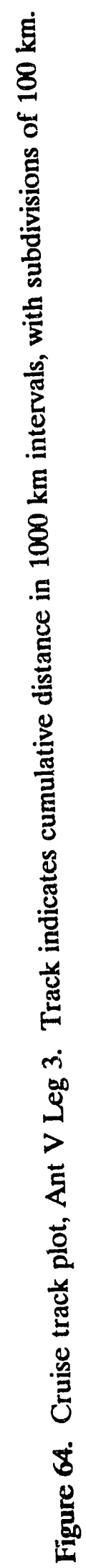




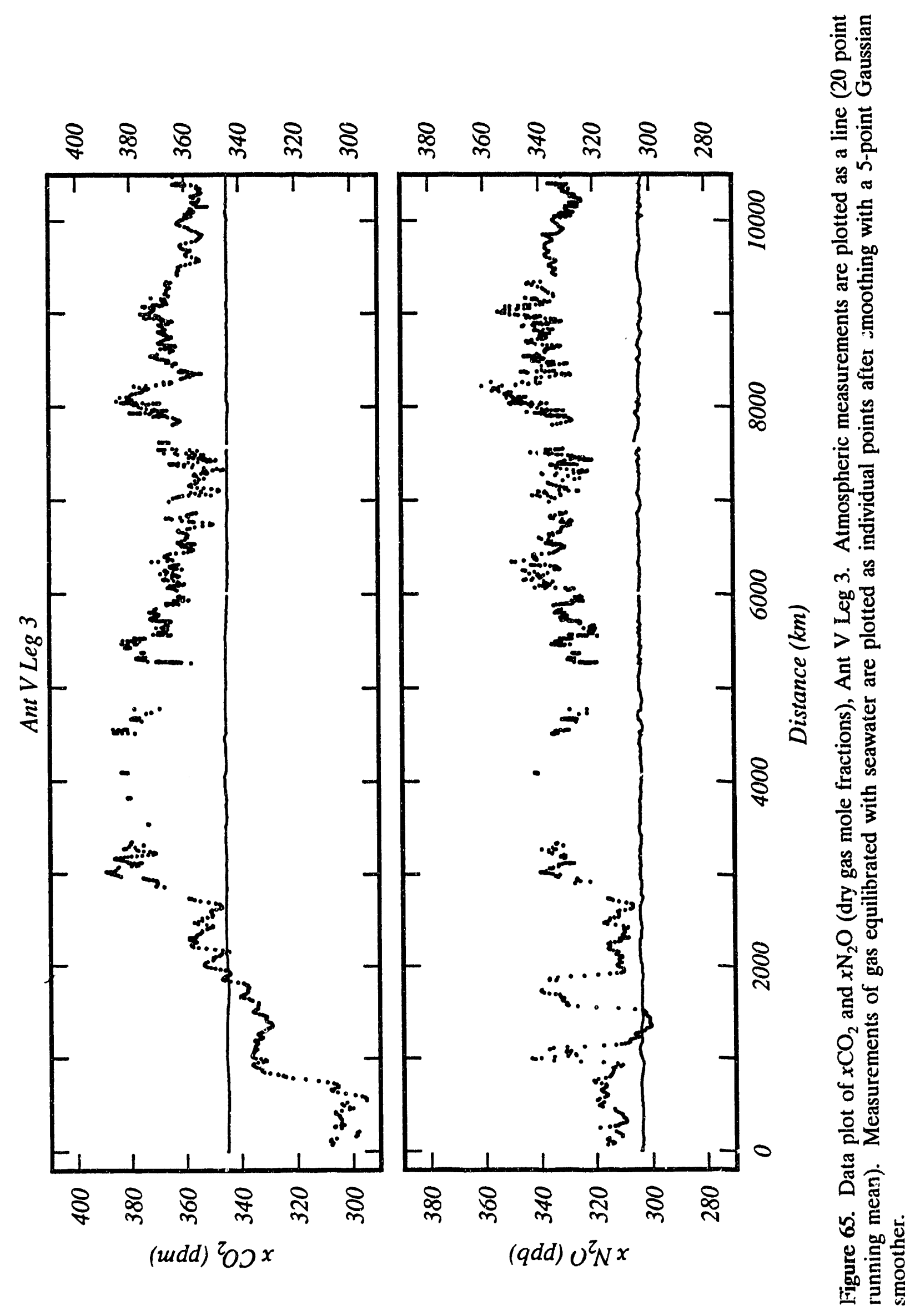




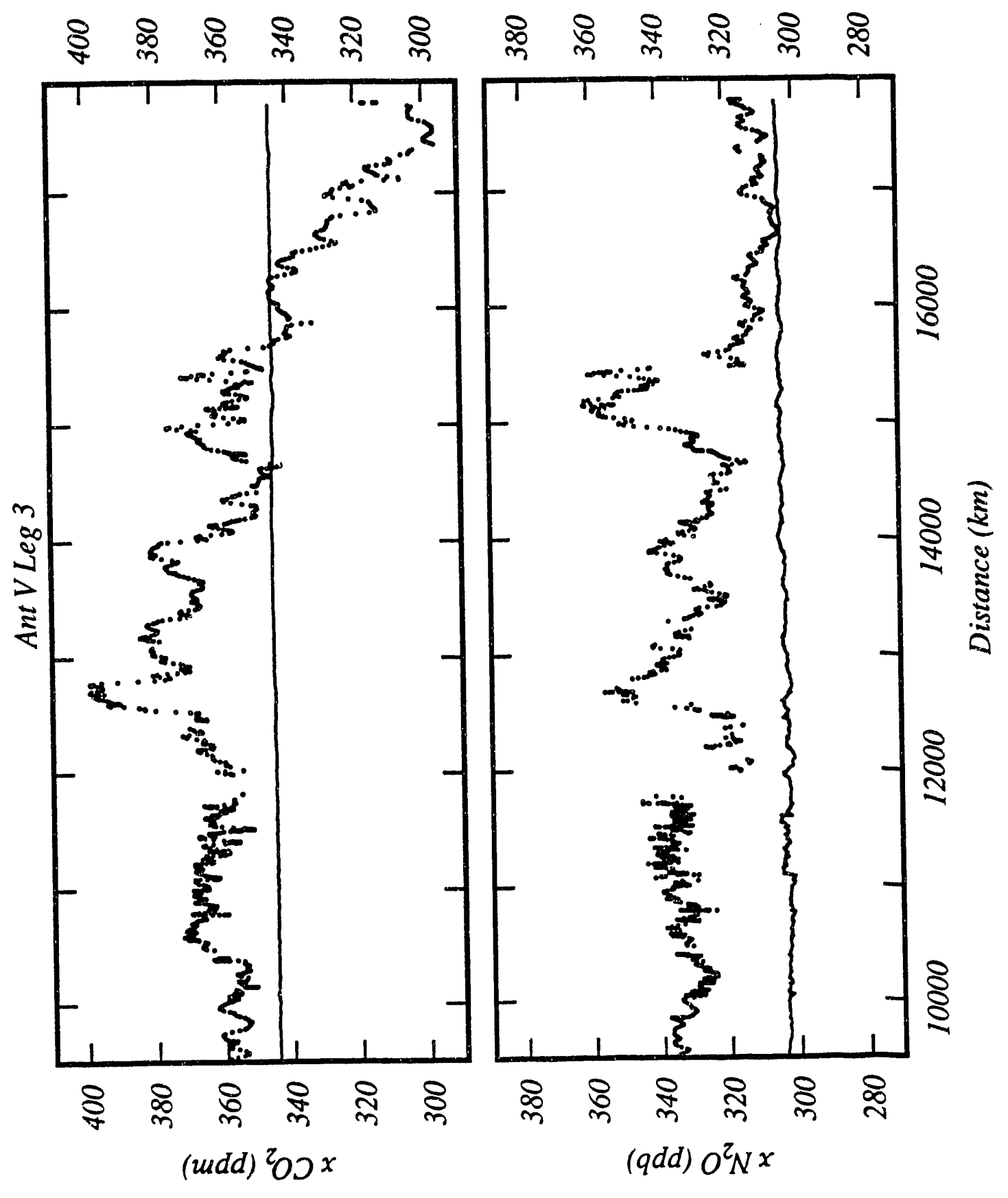

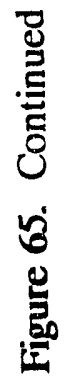




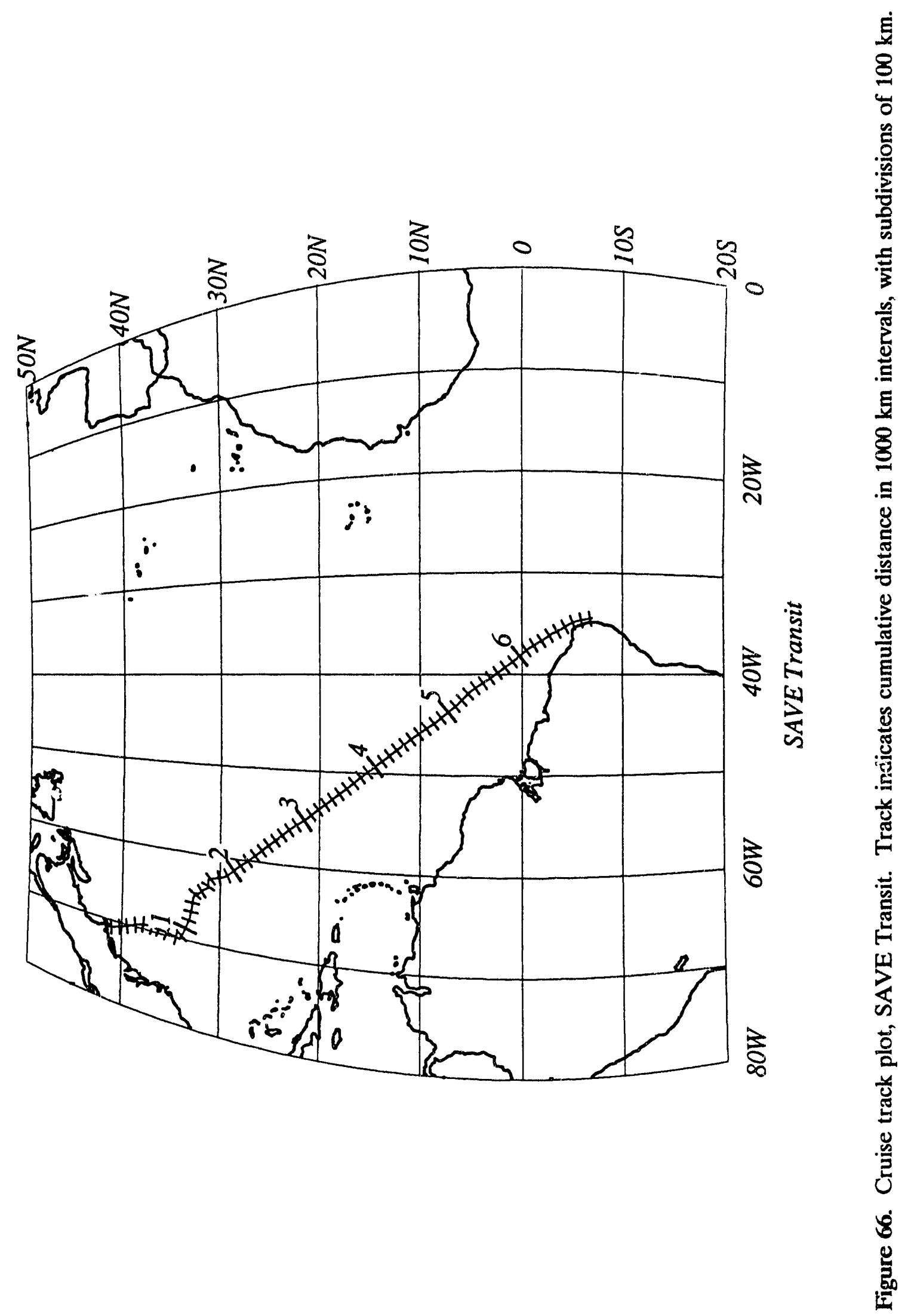




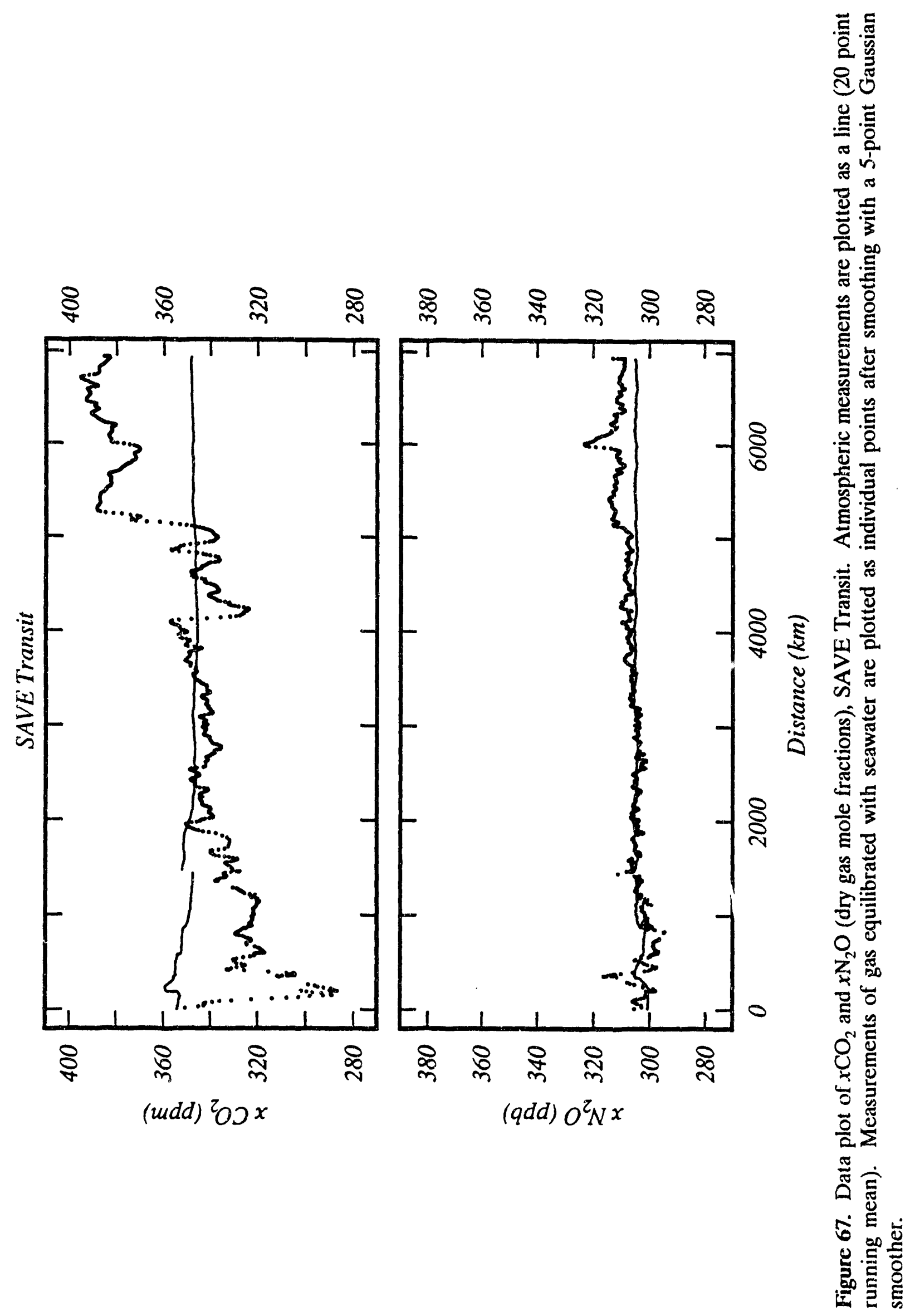




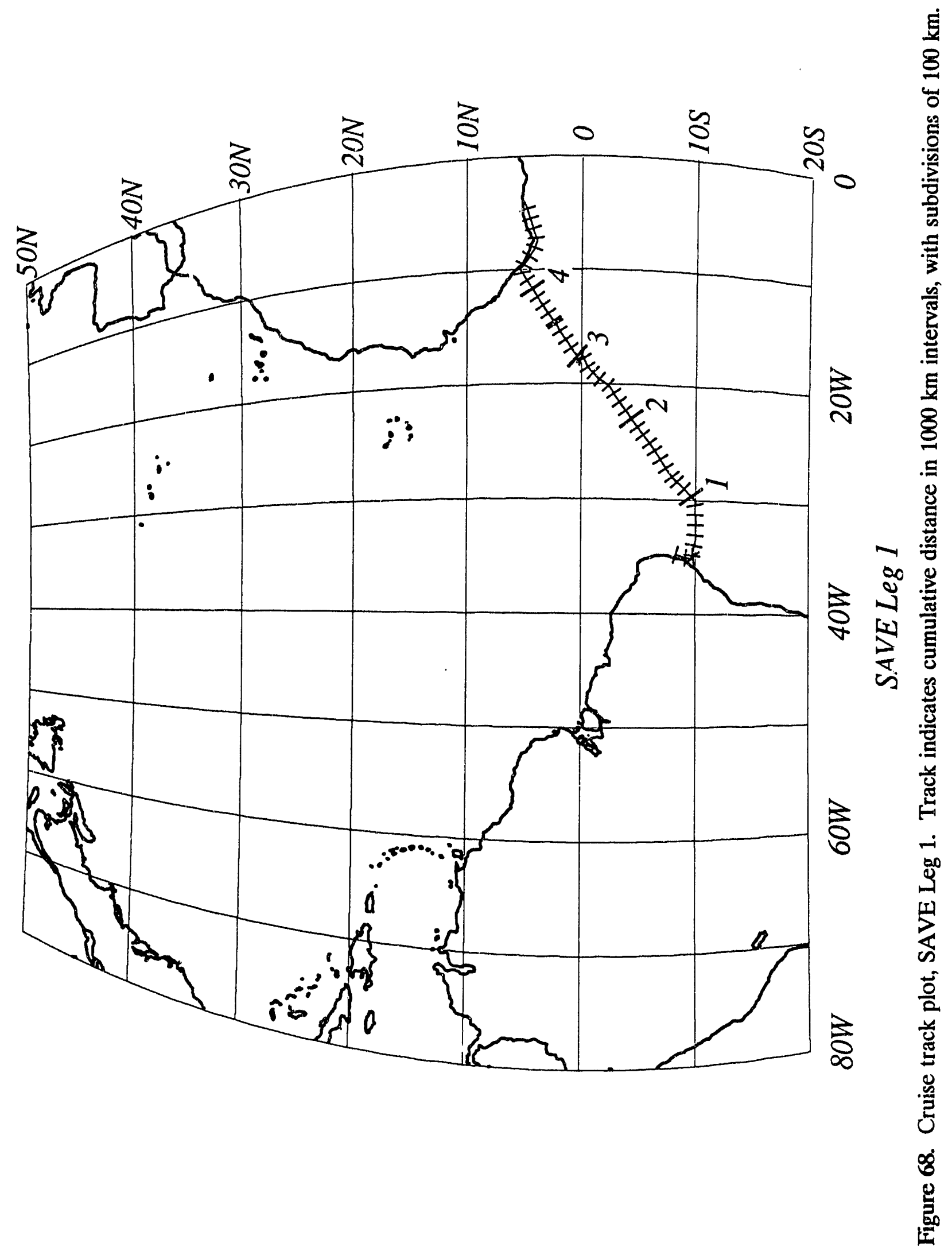




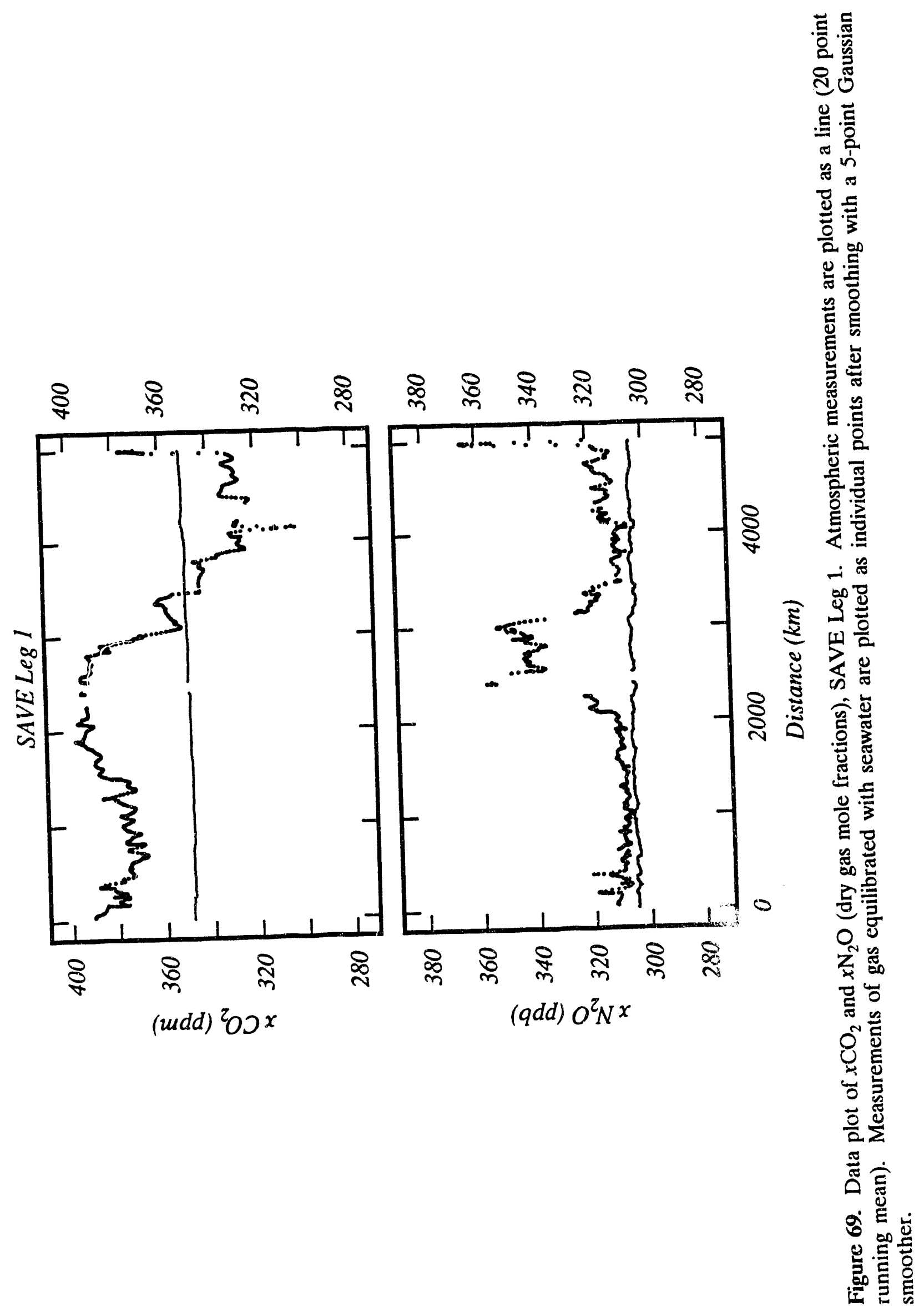




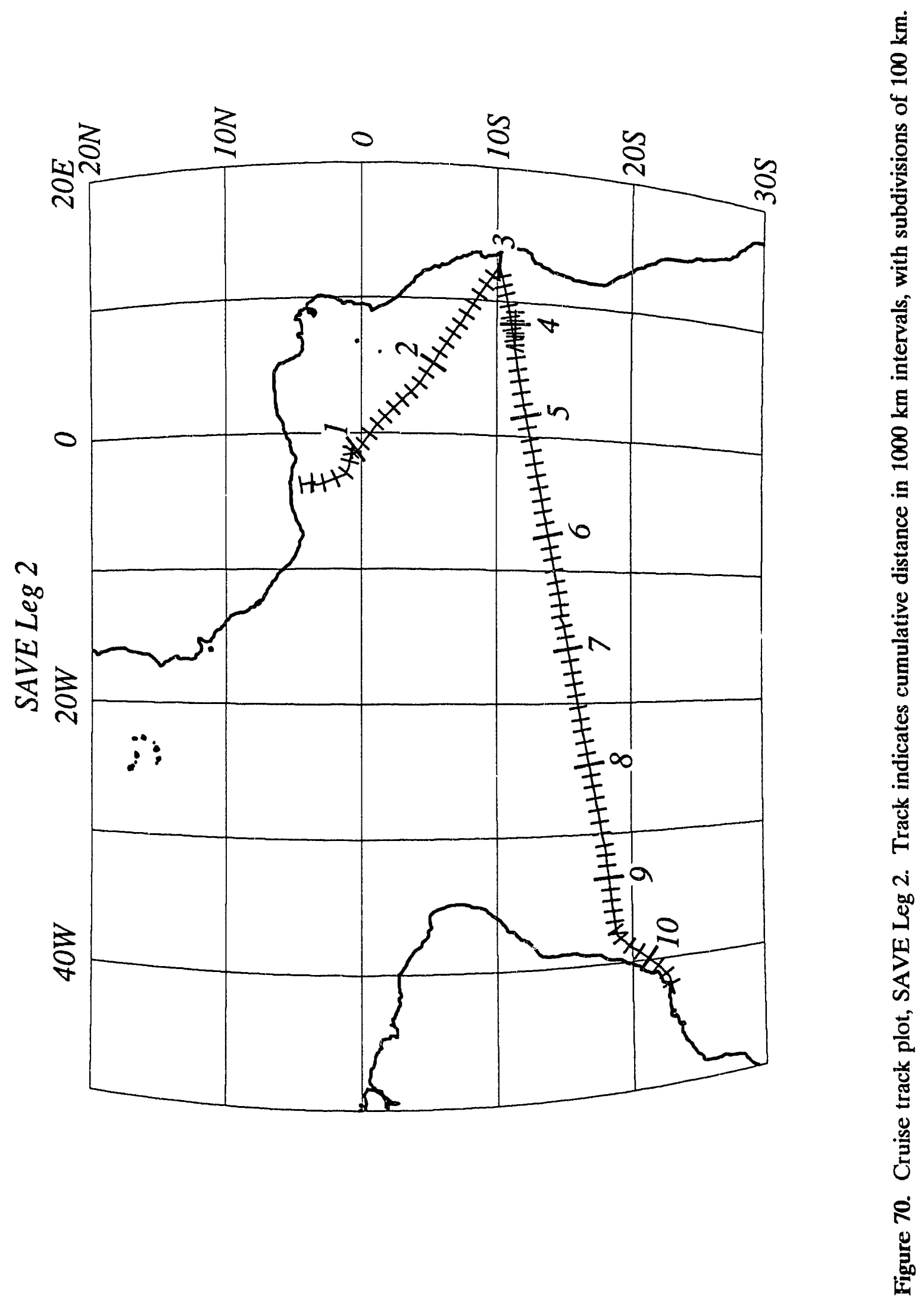




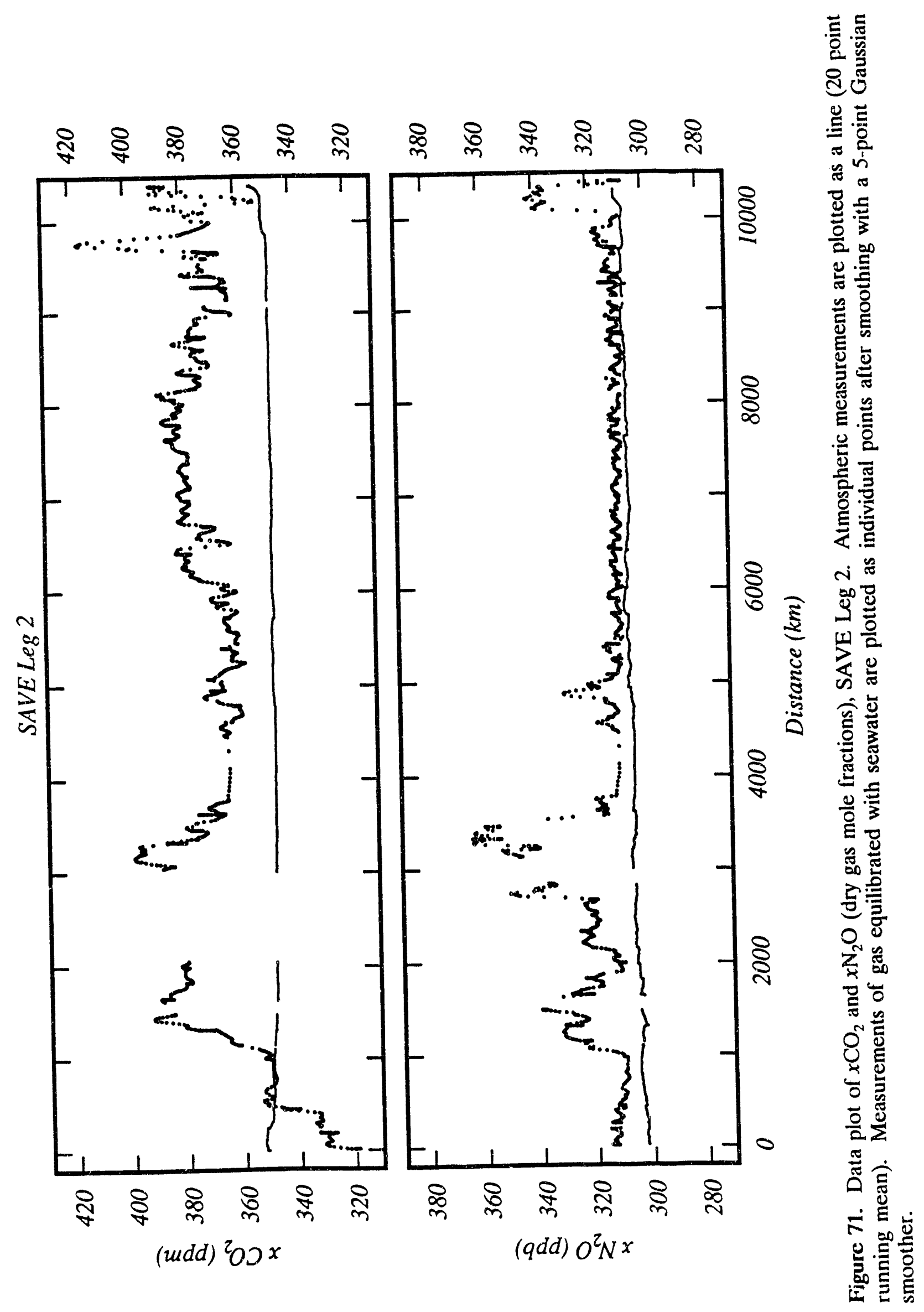




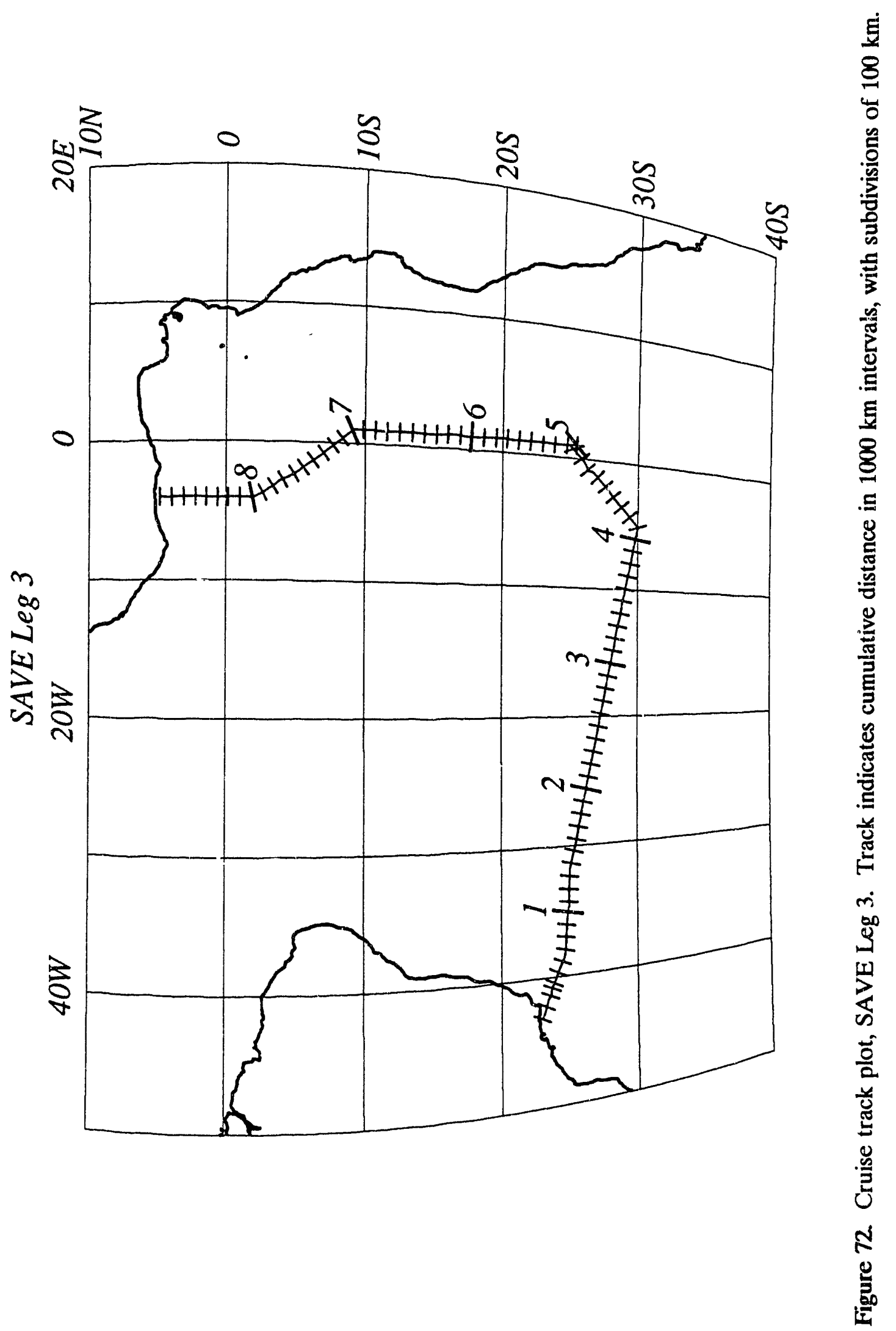




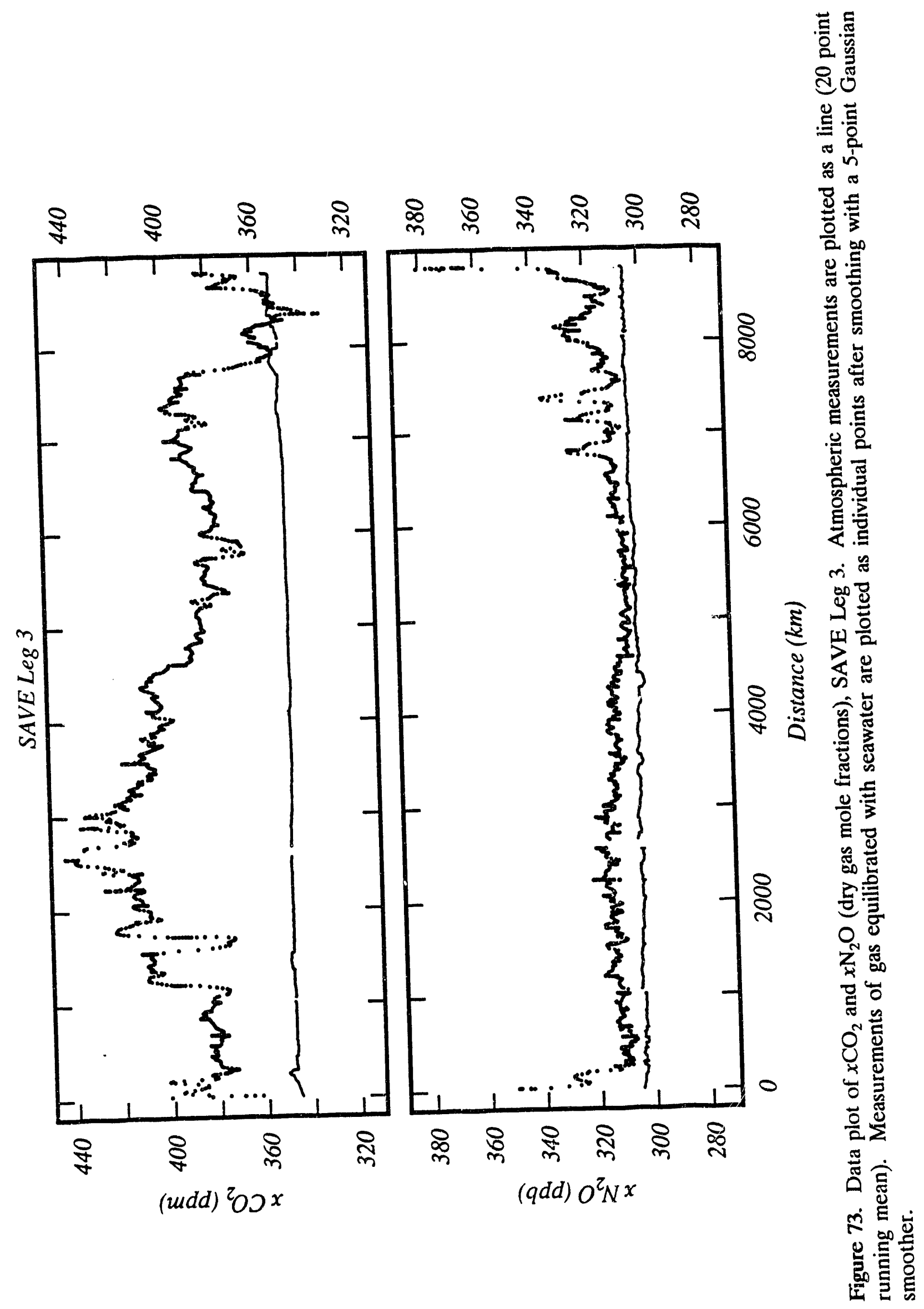




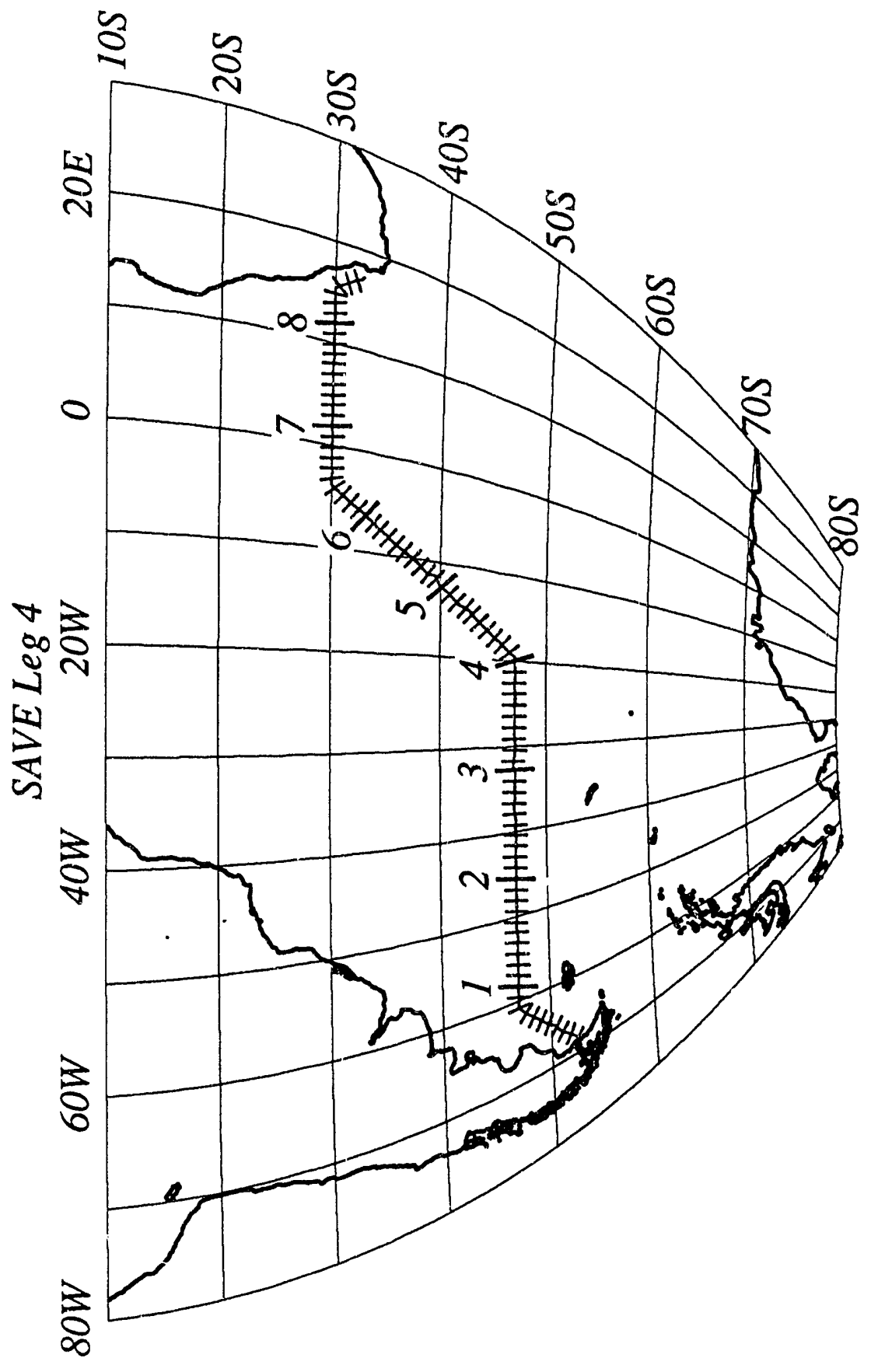

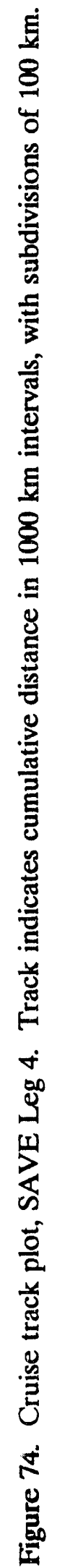




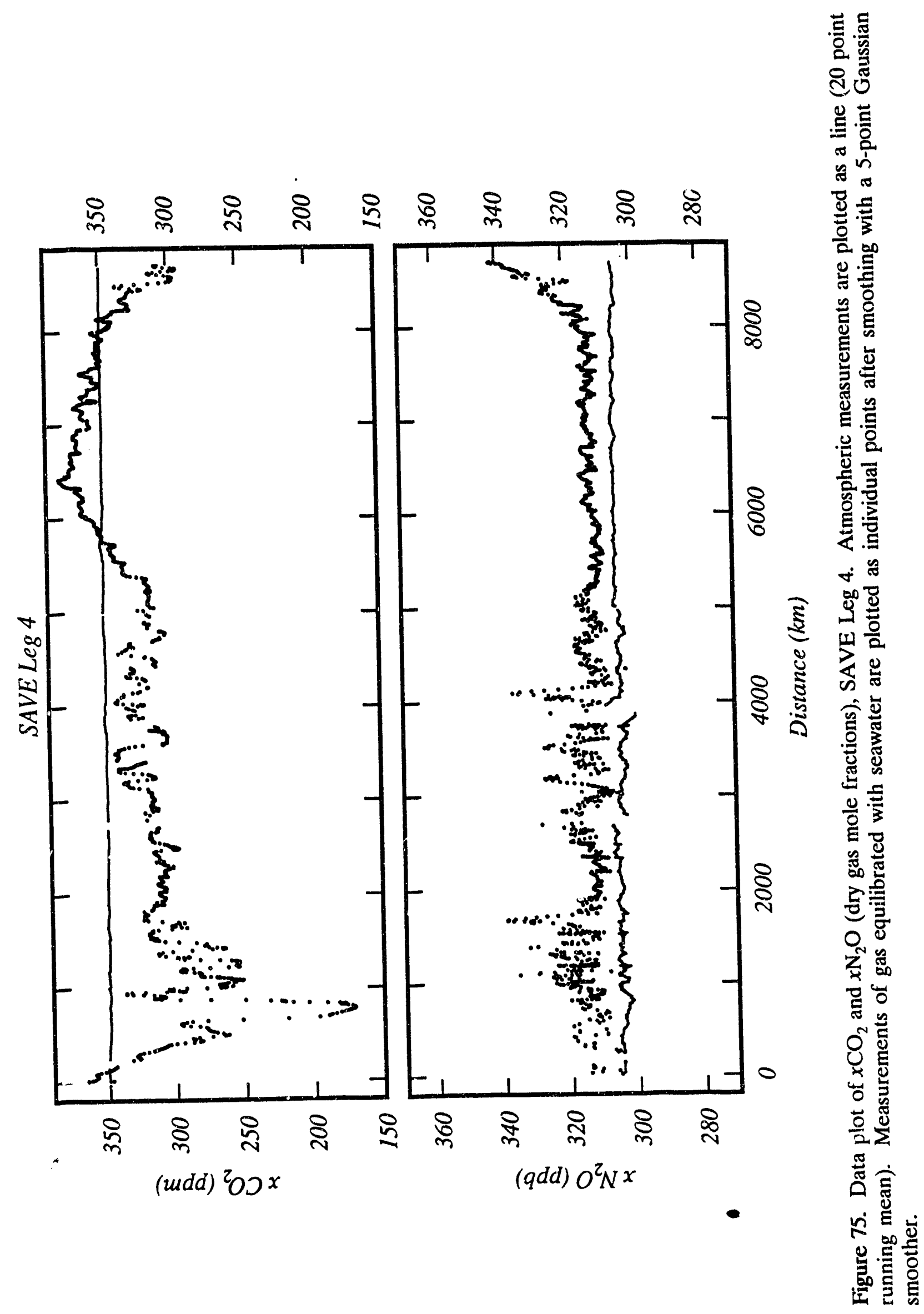




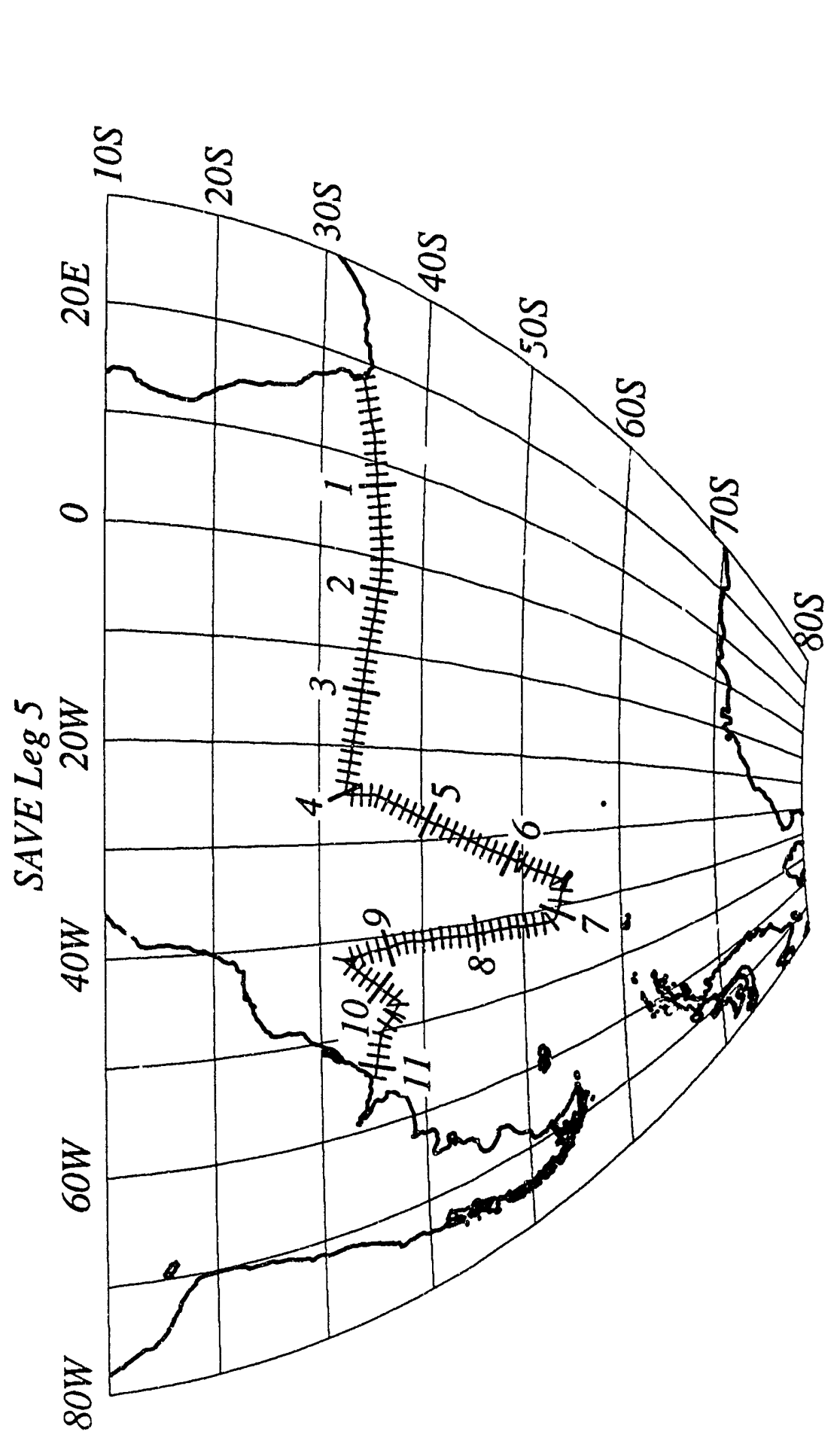

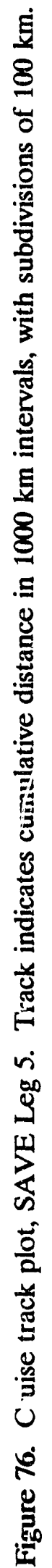




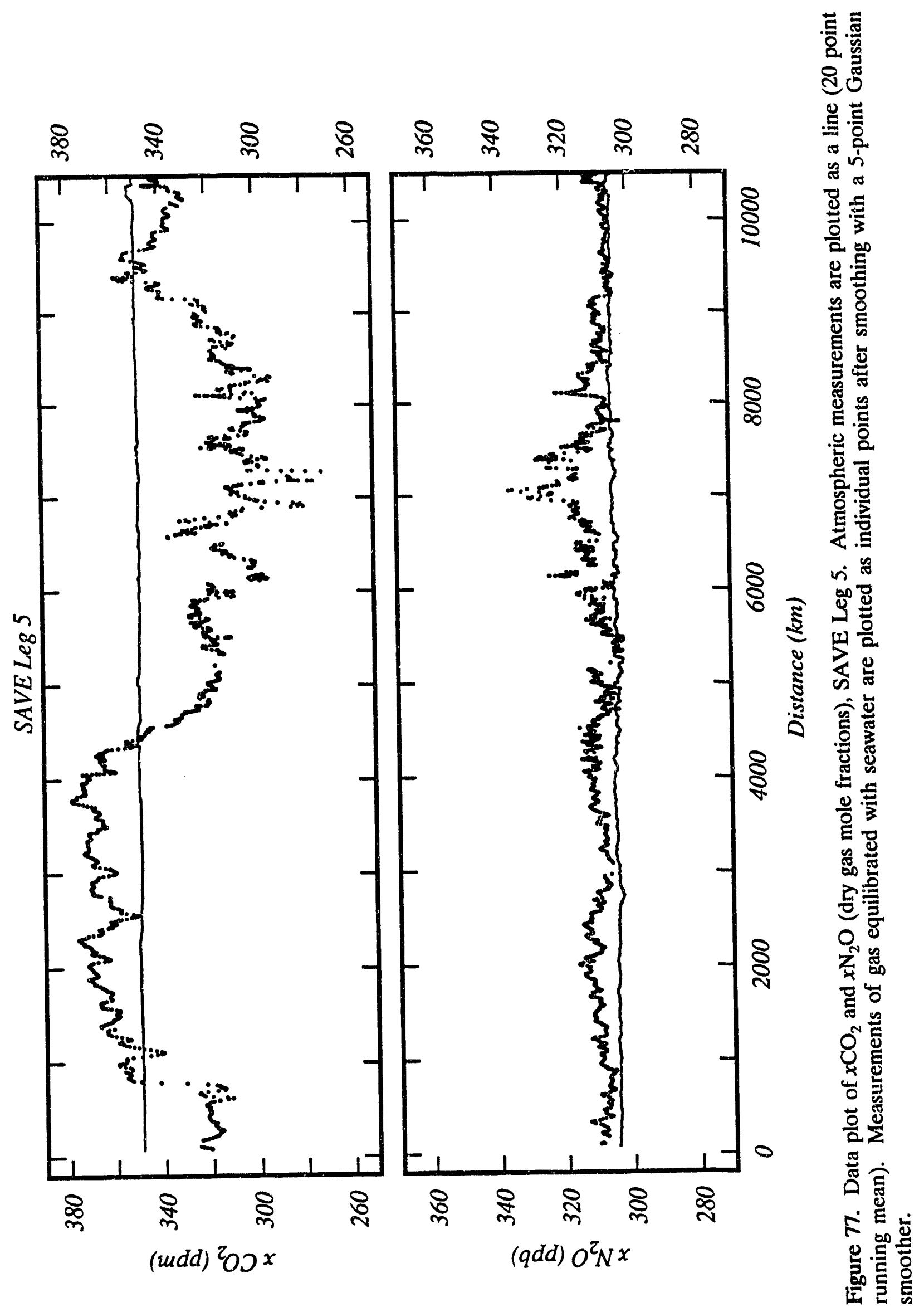




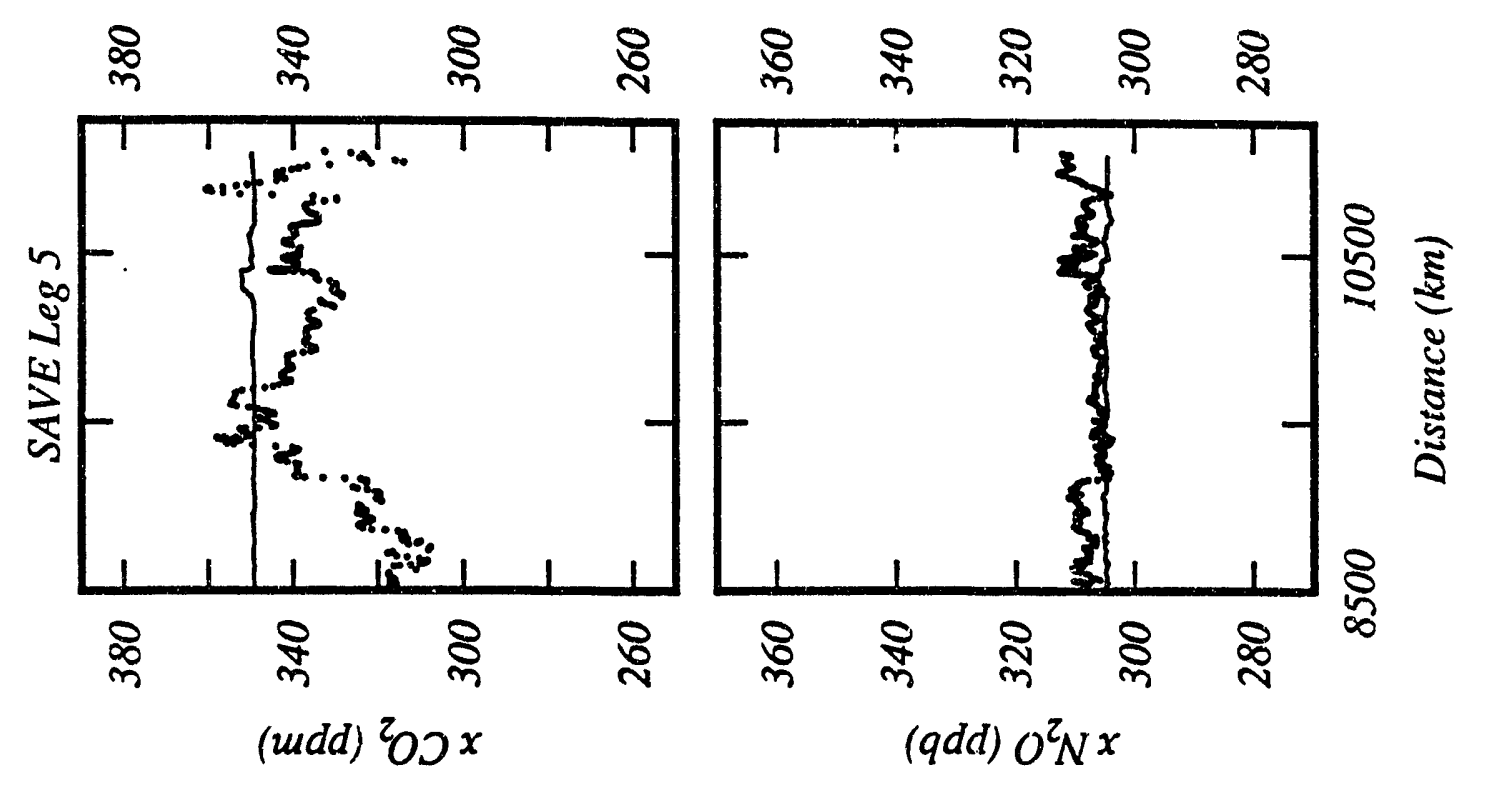

范 


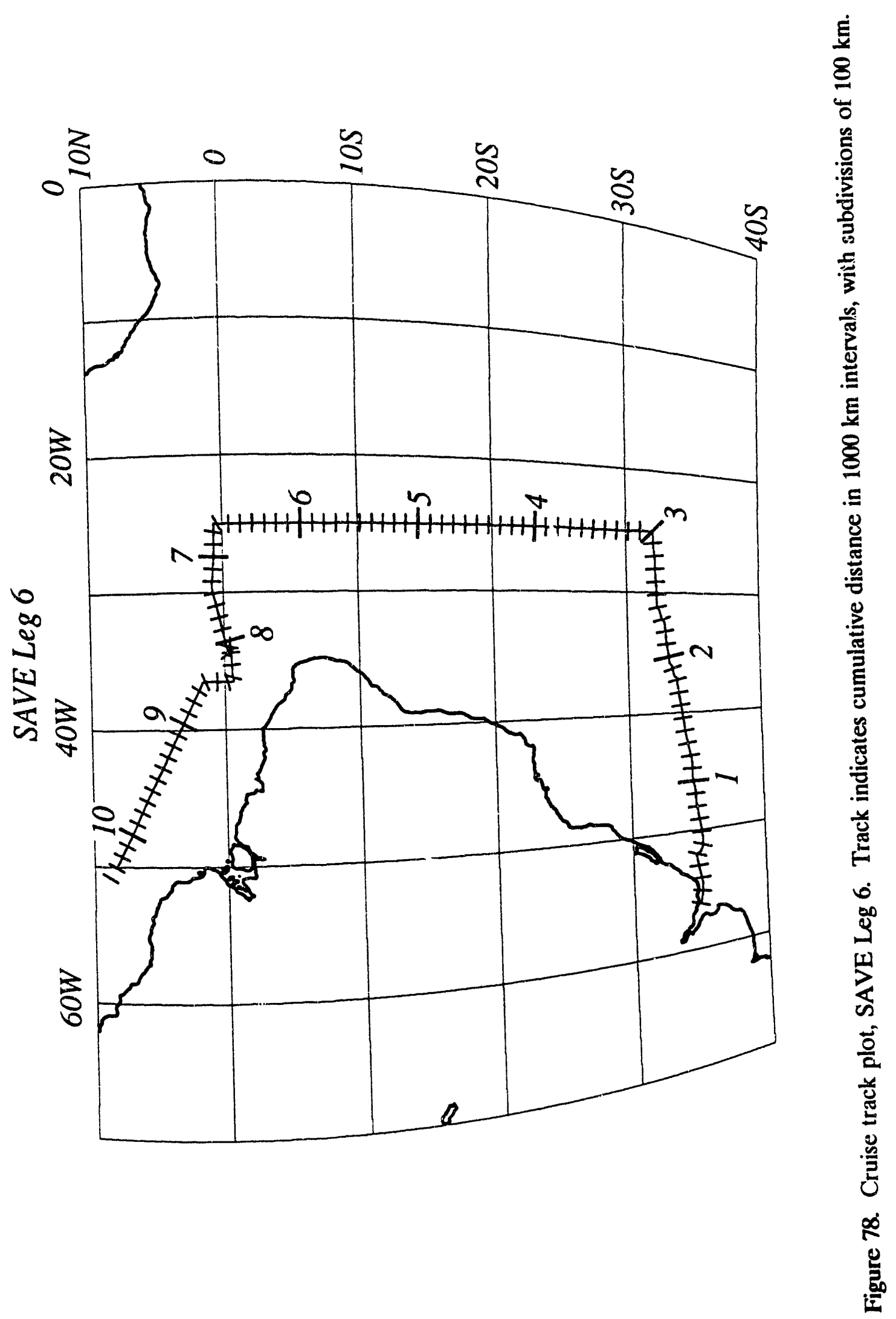




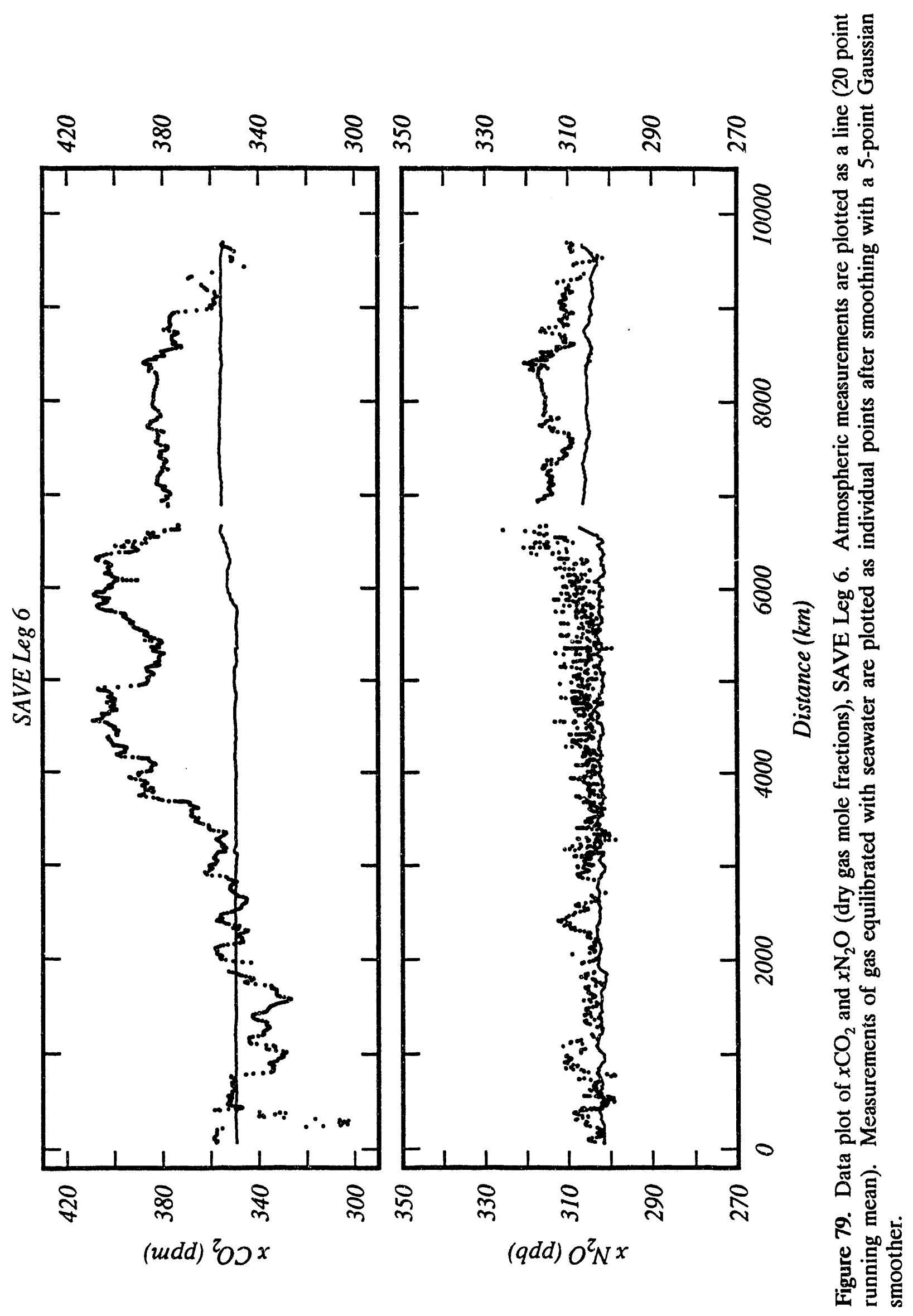




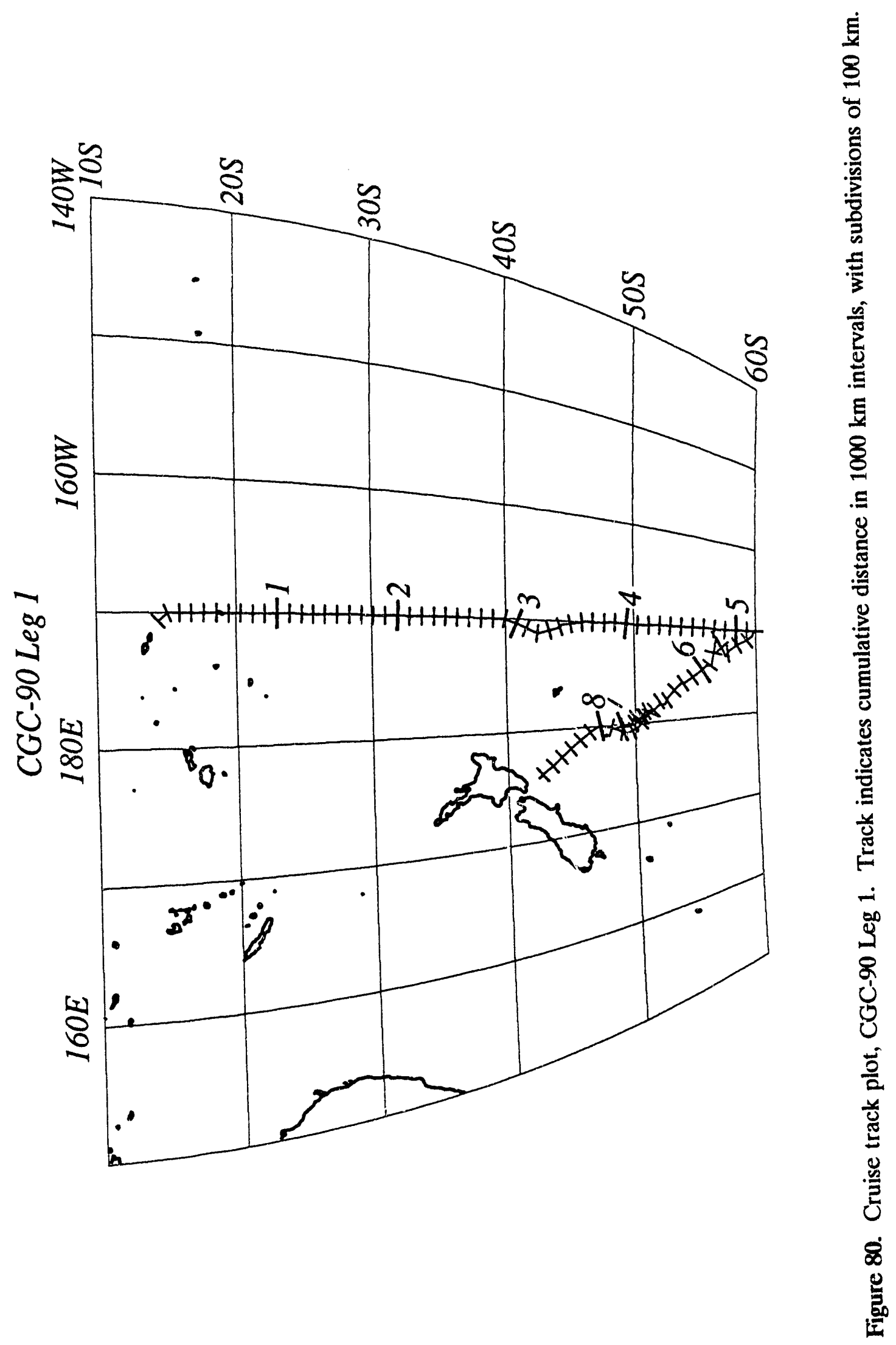




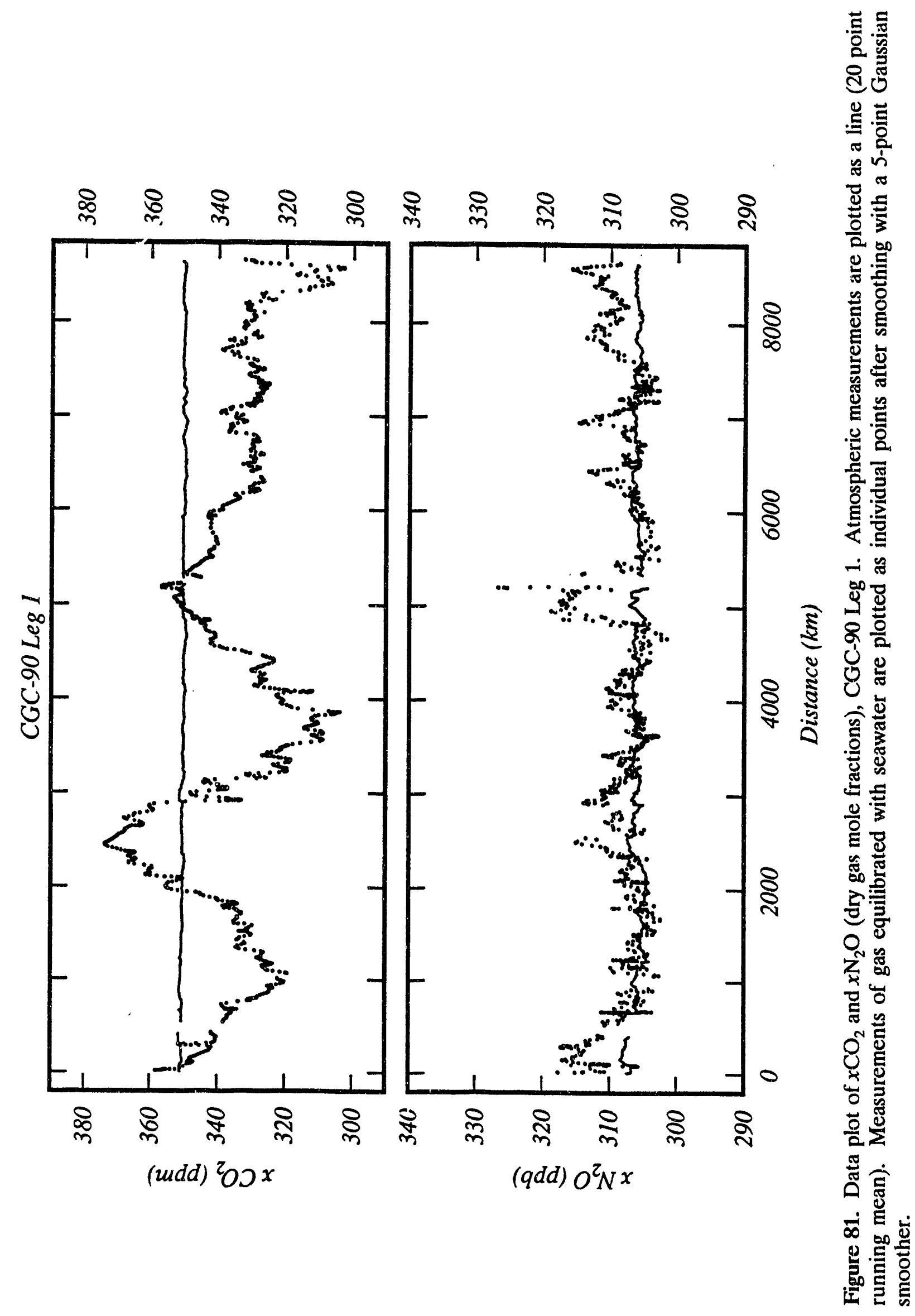



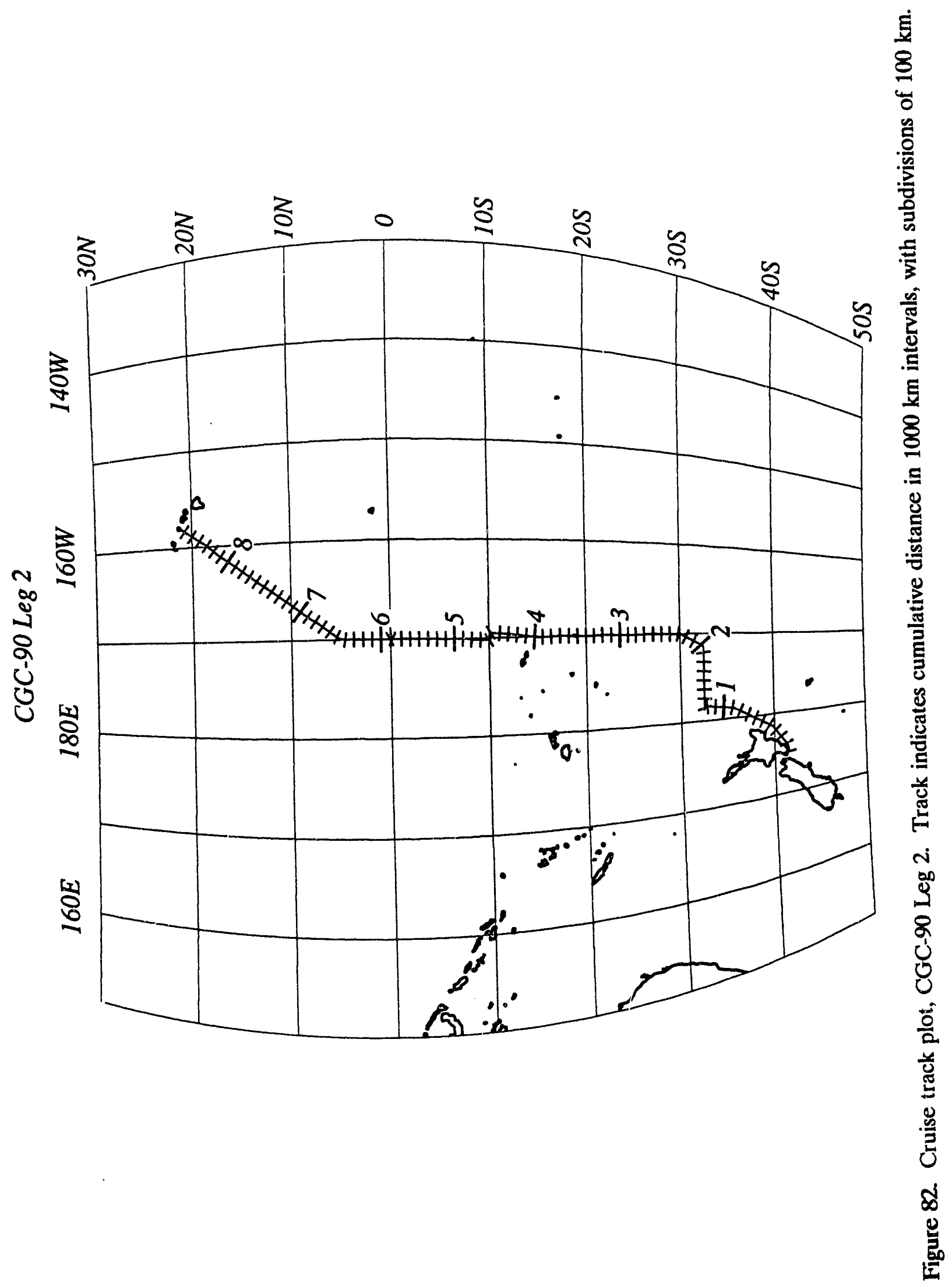


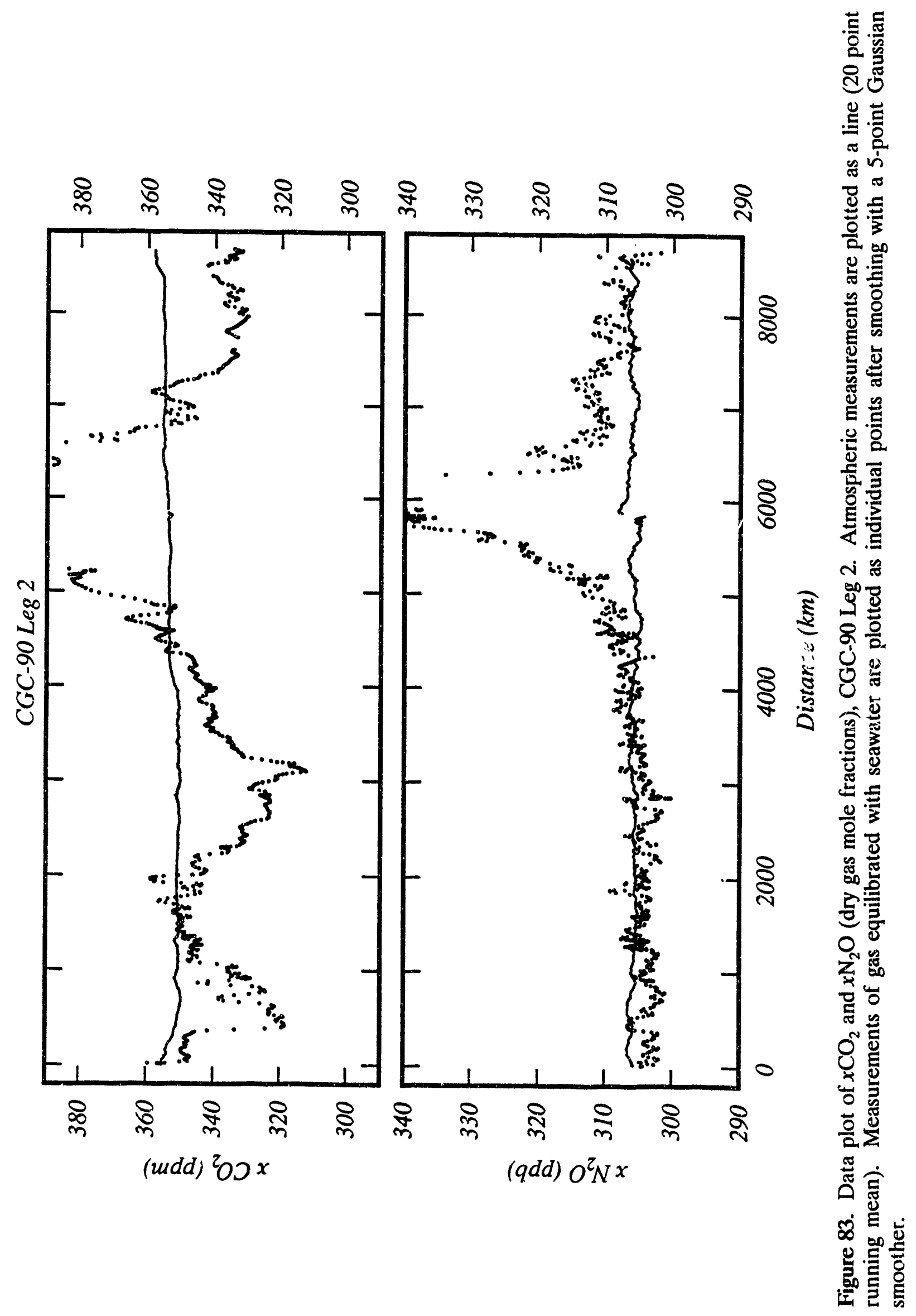




\section{METHODOLOGY}

This document describes the results of surface water and atmospheric $\mathrm{CO}_{2}$ and $\mathrm{N}_{2} \mathrm{O}$ measurements carried out by shipboard gas chromatography over the period 1977-1990. The measurements were made by an automated high-precision shipbcard gas chromatographic system developed during the late 1970 s and used extensively over the intervening years. This instrument, which is described by Weiss (1981), measures $\mathrm{CO}_{2}$ by flame ionization after quantitative reaction to methane in a stream of hydrogen using a reduced nickel catalyst preceded by a palladium catalyst to protect the nickel from oxygen and other atmospheric oxidants. Nitrous oxide is measured by a separate electron capture detector. (Methane is also measured by the flame ionization detector, although the system is not optimized for this gas. The methane results are not included in this data set because methane's equilibration time constant is long, and the results are therefore subject to contamination by biological activity in the ship's seawater pumping system.)

The chromatographic system measures 196 dry-gas samples a day, divided equally among the atmosphere, gas equilibrated with surface water, a low-range gas standard, and a high-range gas standard. Thus, the atmosphere and the ocean are each measured every half-hour, or 48 times a day. This corresponds to a spatial resolution of about $10 \mathrm{~km}$ w'hen the ship is under way and gives several replicate measurements at cach hydrographic station. The typical relative standard deviation of a single determination is about $0.04 \%$ for $\mathrm{CO}_{2}$ and $0.3 \%$ for $\mathrm{N}_{2} \mathrm{O}$, but precision is occasionally affected adversely by shipboard operating conditions. The measurements are calibrated with dry-air secondary standards stored in Spectra-Seal aluminum cylinders. These standards are periodicaliy calibrated for $\mathrm{CO}_{2}$ against the Scripps Institution of Oceanography (SIO) manometric scale in the laboratory of C. $D$. Keeling and for $\mathrm{N}_{2} \mathrm{O}$ against the calibration scale developed by Weiss et al. (1981). In addition to its accuracy, the chromatographic method for $\mathrm{CO}_{2}$ offers the benefits over other commonly used techniques of being independent of oxygen concentration and using small amounts of sample and standard.

Surface seawater is pumped continuously from the sow of the ship (nominal depth approximately $3 \mathrm{~m}$ ) at a rate of about 100 liters $/ \mathrm{min}$. This high pumping rate and the use of plastic polyvinylchloride (PVC) piping assure a minimal change in temperature and a minimal opportunity for chemical alteration of the water. The equilibrator is constructed of heavy acrylic plastic (for visibility and temperature insulation) and has an internal gas volume of about 20 liters. The equilibrator design consists of 2 concentric cylindrical stages, with a drain at the center to minimize volume changes as a result of ship motion. The water "rains" through the 2 stages of the equilibrator at a combined rate of about 20 liters $/ \mathrm{min}$, and a low 0.2-atm pressure head minimizes spraying, bubble entrapment, and other dynamic pressure effects. The 20 -liter gas space is circulated by an air pump through a closed loop which provides the pressurized gas required by the chromatograph. Each half-hourly analysis removes about $75 \mathrm{ml}$ ot gas from this pumped loop. The first siage of the equilibrator is vented to the outside atmosphere so that the gas used for the analysis is replaced by clean marine air.

The temperature of the equilibrator is monitored and compared with the surface ocean temperatures measured $\mathrm{a}^{ \pm}$. hydrographic stations to determine the thermal effect of the ship's pumping system as a function of intake temperature. The maximum amount of change, found for the coldest surface waters, is typically a warming of $<1^{\circ} \mathrm{C}$. As expected, this temperature difference decreases to zero when the water temperature reaches the mean inside temperature of the ship. The measured $\mathrm{CO}_{2}$ values are corrected for this thermal effect (roughly 4\% per degree) using an empirical equation (Weiss et al. 1982) which is dominated 
by the temperature dependence of the $\mathrm{CO}_{2}$ solubility coefficient (Weiss 1974). The measured $\mathrm{N}_{2} \mathrm{O}$ values are also corrected for the solubility effect (Weiss and Price 1980).

The response time of the equilibrator has been evaluated theoretically and experimentally. For unbuffered gases such as nitrous oxide, oxygen and nitrogen, the theoretical response time (assuming complete exchange between water and gas) is given as $F S / V$, where $F$ is the flux of water through the system, $V$ is the volume of the equilibrated gas phase, and $S$ is the Ostwald solubility coefficient. For tine equilibrator used in the measurements presented here, this gives a characteristic (1/e) response time of about $1 \mathrm{~min}$ for $\mathrm{N}_{2} \mathrm{O}$, about $0.5 \mathrm{hr}$ for oxygen and about $1 \mathrm{hr}$ for nitrogen. For $\mathrm{CO}_{2}$ the response time would be similar to $\mathrm{N}_{2} \mathrm{O}$ if there were no chemical buffering, but with chemical buffering (see gas exchange discussion in Broecker and Peng 1982) the response time is enhanced by an order of magnitude to about $0.1 \mathrm{~min}$. Laboratory experiments by Weiss et al. (1982) and by scientists at the National Oceanic and Atmospheric Administration (NOAA), Pacific Marine Environmental Laboratory (PMEL) and Climate Monitoring and Diagnostics Laboratory (CMDL), who have adopted this equilibrator design, have confirmed that the actual equilibration times are close to thes: theoretical values.

These differences in exchange times are important in understanding the performance of an equilibrator that is vented to atmospheric pressure. Since the major components of equilibrated gas - nitrogen, oxygen, and argon - have equilibration times that are much longer than those of the measured species, $\mathrm{N}_{2} \mathrm{O}$ and $\mathrm{CO}_{2}$, the effect will be for the equilibrium partial pressures of these lattur two gases to be present in a water-saturated gas phase at a total pressure equal to the barometric pressure. This is exactly the condition that is satisfied by the actual atmosphere when it is in equilibrium with the ocean, since the gas-phase boundary layer is always saturated with water vapor and at the total barometric pressure. Since the chromatographic system measures the dry-gas mole fractions of these constituents, $x \mathrm{CO}_{2}$ and $x \mathrm{~N}_{2} \mathrm{O}$, in both the atmosphere and the equilibrated gas, and the total pressure is the saine in both cases, the differences in $x \mathrm{CO}_{2}$ between these phasas are a close measure of the differences in $\mathrm{CO}_{2}$ partial pressure $\left(p \mathrm{CO}_{2}\right)$, as long as the total pressure is near 1 atm:

$$
\operatorname{delta}\left(p \mathrm{CO}_{2}\right)=\operatorname{delta}\left(x \mathrm{CO}_{2}\right)\left(P-p \mathrm{H}_{2} \mathrm{O}\right)
$$

where delta signifies the difference between sea and air, $P$ is the barometric pressure, and $\mathrm{pH}_{2} \mathrm{O}$ is the water vapor pressure. Because the temperature and the barometric pressure are routinely recorded, the system is effectively completely constrained, $b$ dt even without these variables delta $\left(x \mathrm{CO}_{2}\right)$ is a very good approximation of delta $\left(p \mathrm{CO}_{2}\right)$. The argument is, of course, the same for the partial pressure of $\mathrm{N}_{2} \mathrm{O}$.

Another question which is more difficult to answer is whether the equilibrium reached by the equilibrator is the true thermodynamic equilibrium that we wish to measure. This type of question is always very difficult to answer, but there is indirect evidence that it is very close. The discrete equilibrator $p \mathrm{CO}_{2}$ measurements carried out by $\mathrm{T}$. Takahashi's group at Lamont-Doherty Geological Observatory during the SAVE expeditions have shown agreement with the equilibrator values presented here to within 1 or $2 \mathrm{ppm}$ ( $\mathrm{T}$. Takahashi, personal communication), even though their measurements are made at a fixed temperature and must be corrected to the surface water temperature. Also, the measurements of $p \mathrm{~N}_{2} \mathrm{O}$ in the central gyres of the major oceans presented here are generally within $1 \%$ of atmospheric saturation. If this were not the correct equilibrium value, one could not explain the constancy of these values over many thousands of kilometers in many different ceniral gyres. Through continued use of the same equilibrator design during the World Ocean 
Circulation Experiment (WOCE), it is hoped to obtain further verification that the measurements are being made at true equilibrium through comparisons with the discrete $p \mathrm{CO}_{2}$ and carbon system measurements being carried out by other lahoratories.

Concentrations of $\mathrm{CO}_{2}$ and $\mathrm{N}_{2} \mathrm{O}$ are calculated by fitting detector peak area response to a quadratic polynomial forced through the origin (zeroth order term is zero). The calculation is performed with the assumption that the linearity of the detector varies slowly compared with changes in detector sensitivity. Accordingly, the second order term of the quadratic polynomial (linearity of the detector) is determined from a running mean of the high standard to low standard response ratio over a range of plus and minus 20 runs, and the first order term (sensitivity of the detector) is determined from the immediately bracketing high and low standard runs. Further details concerning the methods of sample measurement and analysis are provided in Weiss (1981), a copy of which is provided in the appendix of this document.

The magnetic tape (or floppy diskettes) that accompanies this document includes a descriptive information file (File 1 on the magnetic tape or NDP044.DES on the floppy diskettes), a file (File 2 on the magnetic tape or TRACKLST on the floppy diskettes) containing a list of the expedition legs on which measurements were made, and a file (File 3 on the magnetic tape or DATA.LST on the floppy disksttes) containing a list of the corresponding data filenames. The tape or diskettes also contain two data files for each expedition leg: one file containing the atmospheric results and one containing the surface seawater results for $x \mathrm{CO}_{2}$ and $x \mathrm{~N}_{2} \mathrm{O}$ [in parts per million (ppm) and parts per billion (ppb), respectively].

\section{APPLICATIONS OF THE DATA}

The data in this package constitute one of the most extensive records available of $x \mathrm{CO}_{2}$ and, particularly, $x \mathrm{~N}_{2} \mathrm{O}$ in marine air and surface seawater. These data will be useful in modeling applications dealing with the ocean's role in the global biogeochemical cycles of carbon and nitrogen. The combination of atmospheric and surface seawater sampling represented in these data should also make them useful in studies of ocean-atmosphere dynamics. In addition, since determinations of $p \mathrm{CO}_{2}$ in the past were usually derived indirectly, these shipboard gas chromatographic analyses are especially valuable in that they represent direct measurements of seawater $\mathrm{CO}_{2}$ and will be useful in studies evaluating other methodologies for determining $\mathrm{pCO}_{2}$.

\section{LMMTATIONS AND RESTRICTIONS}

The locations, surface water temperatures, and barometric pressures presented in the surface water and atmospheric $\mathrm{CO}_{2}$ and $\mathrm{N}_{2} \mathrm{O}$ data set are all interpolated from the discrete values recorded on the ship, and therefore must be taken only as approximations. As noted in the list of expeditions presented in Table 1 , only $\mathrm{N}_{2} \mathrm{O}$ was measured on the first four expedition legs. Barometric pressure also was not measured on these first four expedition legs, but this should not be a significant impediment to the use of the data for most applications, as was discussed in the methodology section (Section 5). The reader should note that measurements on NORPAX leg 7 and before were made using anhydrous calcium sulfate drying agent to dry the measured samples, which may bias the $\mathrm{CO}_{2}$ results slightly due to 
acid-base reactions. This problem is discussed by Weiss (1981) and affects only these early legs. During the TPS-24 and TPS-47 expeditions, nitrogen carrier gas was used instead of argon-methane carrier gas for the $\mathrm{N}_{2} \mathrm{O}$ determinations. Subsequent comparisons with flask atmospheric samples suggested that this may have produced a small bias of 1 or $2 \mathrm{ppb}$ in the $\mathrm{N}_{2} \mathrm{O}$ results from these legs, but no corrections have been applied.

The primary purpose of these data is to describe large-scale distributions of $\mathrm{CO}_{2}$ and $\mathrm{N}_{2} \mathrm{O}$. The times of the measurements are accurate to within about $1 \mathrm{~min}$, but the ancillary data such as position, sea surface temperature, and barometric pressure were interpolated from observations of extremely variable frequency and accuracy. The uncertainties resulting from these interpolations do not significantly increase the errors in the $\mathrm{CO}_{2}$ and $\mathrm{N}_{2} \mathrm{O}$ results. Ancillary data are reported for the sake of completeness but should not be used in their own right without a more thorough investigation of measurement and interpolation errors.

\section{DATA CHECKS PERFORMED BY CDIAC}

The Carbon Dioxide Information Analysis Center (CDIAC) endeavors to provide quality assurance (QA) of al! data before their distribution. To ensure the highest possible quality in the data, CDIAC conducts extensive reviews for reasonableness, accuracy, completeness, and consistency of form. Although the reviews have common objectives, the specific form must be tailored to each data set; this tiloring process may involve considerable programming efforts. The entire QA process is in impcriant part of CDIAC's effort to ensure accurate, usable $\mathrm{CO}_{2}$-related data for researcisiss.

It is important to emphasize that the data were edited by the authors before submission to CDIAC to remove serious outliers and contaminated samples and to correct gross numerical errors. However, not all the data have yet been subjected to the level of scrutiny associated with careful interpretive work. Readers are therefore requested to draw to the attention of the authors any suspected inconsistencies in these data. Readers who have obtained this report directly from CDIAC will automatically receive notification of updates and corrections. The authors also wish to encourage scientific collaborations with readers for the purpose of interpreting the results of these observations.

The following summarizes the QA checks performed on the surface water and atmospueric $\mathrm{CO}_{2}$ and $\mathrm{N}_{2} \mathrm{O}$ data by CDIAC.

1. The format of all information, including header items, was checked to ensure consistency throughout each data file.

2. All numeric values were inspected for logical inconsistencies (e.g., values of DATEDA $<1$ or $>31$; YEAR $<1977$ or $>1990$; TIME $<0$ or $>2359$; LAT $<-90.0$ or $>90.0$; LON $<-180.0$ or $>180.0$ ) and for the presence of outliers (e.g., PRESS $<900$ or $>1100$; H2OTMP $<-5$ or $>32$ ).

The data distributed in this package are identical to the original data received by CDIAC. However, in order to enhance the ease of use of these data, the following alterations in format were made.

1. The data filenames were modified to conform to the two-level naming convention of DOS-based systems; for example, 05.INDOMED.LEG11A.WATER was changed to 
INDOM11A.H2O. A complete listing of the filenames and the corresponding expedition legs is given in Section 11 of this document.

2. Within each data file, all header material was condensed into a single line. This involved removing blank lines a atmospheric data or surface seawater data). In addition, all descriptive column tixies were removed.

3. Values of latitude and longitude were converted from degrees and minutes to decimal degrees, and signs were added to denote the hemisphere (Northern and Eastern Hemispheres were assigned " + " values; Southern and Western Hemispheres were assigned "-" values).

4. The designations for missing values, given as blanks in the original files, were changed to the following: $\mathbf{- 9 9 9 . 9}$ for missing values of barometric pressure; 99.99 for missing values of surface water temperature; and -99.9 for missing values of $x \mathrm{~N}_{2} \mathrm{O}$ and $x \mathrm{CO}_{2}$.

\section{REFERENCES}

Broecker, W. S., and T.-H. Peng. 1982. Tracers in the Sea. Eldigio Press, Lamont-Doherty Geological Observatory of Columbia University, Palisades, New York.

Weiss, R. F. 1974. Carbon dioxide in water and seawater: The solubility of a non-ideal gas. Marine Chemistry 2:203-215.

Weiss, R. F., and B. A. Price. 1980 . Nitrous oxide solubility in water and seawater. Marine Chemistry 8:347-359.

Weiss, R. F. 1981. Determinätions of carbon dioxide and methane by dual catalyst flame ionization chromatography and nitrous oxide by electron capture chromatography. Journal of Chromatographic Science 19:611-616.

Weiss, R. F., C. D. Keeling, and H. Craig. 1. 81. The determination of tropospheric nitrous oxide. Journal of Geophysical Research 86:7197-7202.

Weiss, R. F., R. A. Jahnke, and C. D. Keeling. 1982. Seasonal effects of temperature and salinity on the partial pressure of carbon dioxide in seawater. Nature 300:511-513.

\section{HOW TO OBTAIN THE DATA PACKAGE}

This document describes a data set consisting of surface water and atmospheric $\mathrm{CO}_{2}$ and $\mathrm{N}_{2} \mathrm{O}$ measurements carried out by shipboard gas chromatography over the period 1977-1990. The data are available without charge upon request on nine-track magnetic tape, on floppy diskettes (IBM PC format, high- or low-density, 5.25- or 3.5-in. diskettes), or through File Transfer Protocol (FTP) from CDIAC. Requests for magnetic tapes should include any 
specific instructions for transmitting the data as required by the user to access the data. Requests not accompanied by specific instructions will be filled on nine-track, $6250 \mathrm{BPI}$, standard-labeled tapes with characters written in Extended Binary Codes Decimal Interchange Code (EBCTIC), and files will be formatted as given in Section 11. Requests should be addressed to the following.

Carbon Dioxide Information Analysis Center

Oak Ridge National Laboratory

Post Office Box 2008

Oak Ridge, Tennessee 37831-6335

U.S.A.

The tape and documentation can be ordered by telephone, fax, or through electronic mail.

Telephone: (615) 574-0390

Fax: $\quad(615) \quad 574-2232$

Electronic mail: BITNET: CDP@ORNLSTC

OMNET: CDIAC

INTERNET: CDP@ORNL.GOV 
PART 2

INFORMATION ABOUT THE DATA FILES PROVIDED ON MAGNETIC TAPE OR FLOPPY DISKETTES 


\section{CONTENTS OF THE MAGNETIC TAPE OR FLOPPY DISKETTES}

The following is a list of files distributed on magnetic tape or floppy diskettes by CDIAC along with this documentation.

File number, diescription, and name

\section{Logical}

records
Record

format $^{a}$
Block

size
Record

length

1. General descriptive information file:

NDP044.DES

324

FB

8000

80

2. Track list of expedition legs: TRACK.LST

3. List of data filenames:

DATALST

4. FORTRAN-77 data retrieval code to read and print the surface water and atmospheric $\mathrm{CO}_{2}$ and $\mathrm{N}_{2} \mathrm{O}$ data (Files 5-86): NDP044.FOR

5. $\mathrm{SAS}^{b}$ input/output routine to read and print the surface water and atmospheric $\mathrm{CO}_{2}$ and $\mathrm{N}_{2} \mathrm{O}$ data (Files 5-86): NDP044.SAS

6. Atmospheric $\mathrm{CO}_{2}$ and $\mathrm{N}_{2} \mathrm{O}$ data for Indomed Leg 2: INDOM2.AIR

7. Surface seawater $\mathrm{CO}_{2}$ and $\mathrm{N}_{2} \mathrm{O}$ data for Indomed Leg 2 : INDOM2.H2O

8. Atmospheric $\mathrm{CO}_{2}$ and $\mathrm{N}_{2} \mathrm{O}$ data for Indomed Leg 3:

9. Surface seawater $\mathrm{CO}_{2}$ and $\mathrm{N}_{2} \mathrm{O}$ data for Indomed Leg 3: INDOM3.H2O

10. Atmospheric $\mathrm{CO}_{2}$ and $\mathrm{N}_{2} \mathrm{O}$ data for Indomed Leg 4: INDOM4.AIR 
File number, description, and name
Logical

records
Record

format $^{\circ}$
Block

size
Record

length

11. Surface seawater $\mathrm{CO}_{2}$ and

$\mathrm{N}_{2} \mathrm{O}$ data for Indomed $\mathrm{Leg} 4$ :

INDOM4.H2O

1529

FB

8000

80

12. Atmospheric $\mathrm{CO}_{2}$ and $\mathrm{N}_{2} \mathrm{O}$

data for Indomed Leg 5:

INDOM5.AIR

1233

FB

8000

80

13. Surface seawater $\mathrm{CO}_{2}$ and

$\mathrm{N}_{2} \mathrm{O}$ data for Indomed Leg 5:

INDOM5.H2O

1223

FB

8000

80

14. Atmospheric $\mathrm{CO}_{2}$ and $\mathrm{N}_{2} \mathrm{O}$ data for Indomed Leg 11A: INDOM11A.AIR

FB

8000

80

15. Surface seawater $\mathrm{CO}_{2}$ and $\mathrm{N}_{2} \mathrm{O}$ data for Indomed Leg 11A: INDOM11A.H2O

FB

8000

80

16. Atmospheric $\mathrm{CO}_{2}$ and $\mathrm{N}_{2} \mathrm{O}$ data for Indomed Leg 12: INDOM12.AIR

FB

8000

80

17. Surface seawater $\mathrm{CO}_{2}$ and $\mathrm{N}_{2} \mathrm{O}$ data for Indomed Leg 12: INDOM12.H2O

FB

8000

80

18. Atmospheric $\mathrm{CO}_{2}$ and $\mathrm{N}_{2} \mathrm{O}$ data for Indomed Leg 15 : INDOM15.AIR

8000

80

19. Surface seawater $\mathrm{CO}_{2}$ and $\mathrm{N}_{2} \mathrm{O}$ data for Indomed Leg 15: INDOM15.H2O

8000

80

20. Atmospheric $\mathrm{CO}_{2}$ and $\mathrm{N}_{2} \mathrm{O}$ data for Indomed Leg $15 \mathrm{~A}$ : INDOM15A.AIR

FB

8000

80

21. Surface seawater $\mathrm{CO}_{2}$ and $\mathrm{N}_{2} \mathrm{O}$ data for Indomed Leg $15 \mathrm{~A}$ : INDOM15A.H2O

22. Atmospheric $\mathrm{CO}_{2}$ and $\mathrm{N}_{2} \mathrm{O}$ data for NORPAX Transit: NORPAX0.AIR

23. Surface seawater $\mathrm{CO}_{2}$ and $\mathrm{N}_{2} \mathrm{O}$ data for NORPAX Transit: NORPAX0.H2O

24. Atmospheric $\mathrm{CO}_{2}$ and $\mathrm{N}_{2} \mathrm{O}$ data for NORPAX Leg 7: NORPAX7.AIR 
File number, description, and name
Logical records
Record

format
Block

size
Record length

25. Surface seawater $\mathrm{CO}_{2}$ and $\mathrm{N}_{2} \mathrm{O}$ data for NORPAX Leg 7: NORPAX7.H2O

26. Atmospheric $\mathrm{CO}_{2}$ and $\mathrm{N}_{2} \mathrm{O}$ data for NOPPAX Leg 9: NORPAX9.AIR

27. Surface seawater $\mathrm{CO}_{2}$ and $\mathrm{N}_{2} \mathrm{O}$ data for NORPAX Leg 9: NORPAX9.H2O

28. Atmospheric $\mathrm{CO}_{2}$ and $\mathrm{N}_{2} \mathrm{O}$ data for NORPAX Leg 13: NORF'AX13.AIR

29. Surface seawater $\mathrm{CO}_{2}$ and $\mathrm{N}_{2} \mathrm{O}$ data for NORPAX Leg 13: NORPAX13.H2O

30. Atmospheric $\mathrm{CO}_{2}$ and $\mathrm{N}_{2} \mathrm{O}$ data for NORPAX Leg 15: NORPAX15.AIR

31. Surface seawater $\mathrm{CO}_{2}$ and $\mathrm{N}_{2} \mathrm{O}$ data for NORPAX Leg 15: NORPAX15.H2O

32. Atmospheric $\mathrm{CO}_{2}$ and $\mathrm{N}_{2} \mathrm{O}$ data for TTO/NAS Leg 1: NAS1.AIR

33. Surface seawater $\mathrm{CO}_{2}$ and $\mathrm{N}_{2} \mathrm{O}$ data for TTO/NAS Leg 1: NAS1.H2O

34. Atmospheric $\mathrm{CO}_{2}$ and $\mathrm{N}_{2} \mathrm{O}$ data for TTO/NAS Leg 2: NAS2.AIR

35. Surface seawater $\mathrm{CO}_{2}$ and $\mathrm{N}_{2} \mathrm{O}$ data for TTO/NAS Leg 2: NAS2.H12O

36. Atmosplieric $\mathrm{CO}_{2}$ and $\mathrm{N}_{2} \mathrm{O}$ data for TTO/NAS Leg 3: NAS3.AIR

37. Surface seawater $\mathrm{CO}_{2}$ and $\mathrm{N}_{2} \mathrm{O}$ data for TTO/NAS Leg 3: NAS3.H2O

38. Atmospheric $\mathrm{CO}_{2}$ and $\mathrm{N}_{2} \mathrm{O}$ data for TTO/NAS Leg 4: NAS4.AIR 
File number, description, and name

\section{Logical}

records
Record format ${ }^{a}$
Block

size
Record

length

39. Surface seawater $\mathrm{CO}_{2}$ and

$\mathrm{N}_{2} \mathrm{O}$ data for TTO/NAS Leg 4:

NAS4.H2O

933

FB

8000

80

40. Atmospheric $\mathrm{CO}_{2}$ and $\mathrm{N}_{2} \mathrm{O}$

data for TTO/NAS Leg 5:

NAS5.AIR

1206

FB

8000

80

41. Surface seawater $\mathrm{CO}_{2}$ and

$\mathrm{N}_{2} \mathrm{O}$ data for TTO/NAS Leg 5:

NAS5.H2O

1109

FB

8000

80

42. Atmospheric $\mathrm{CO}_{2}$ and $\mathrm{N}_{2} \mathrm{O}$ data for TTO/NAS Leg 6:

NAS6.AIR

1255

FB

8000

80

43. Surface seawater $\mathrm{CO}_{2}$ and

$\mathrm{N}_{2} \mathrm{O}$ data for TTO/NAS Leg 6: NAS6.H2O

1212

FB

8000

80

44. Atmospheric $\mathrm{CO}_{2}$ and $\mathrm{N}_{2} \mathrm{O}$

data for TTO/NAS Leg 7:

NAS7.AIR

1144

FB

8000

80

45. Surface seawater $\mathrm{CO}_{2}$ and

$\mathrm{N}_{2} \mathrm{O}$ data for TTO/NAS Leg 7:

NAS7.H2O

1089

FB

8000

80

46. Atmospheric $\mathrm{CO}_{2}$ and $\mathrm{N}_{2} \mathrm{O}$

data for Hudson 82-001 Leg 1:

HUD1.AIR

1578

FB

8000

80

47. Surface seawater $\mathrm{CO}_{2}$ and $\mathrm{N}_{2} \mathrm{O}$ data $f_{0}$ - Hudson 82-001 Leg 1 : HUC : 20

8000

80

48. Atmospheric $\mathrm{CO}_{2}$ and $\mathrm{N}_{2} \mathrm{O}$ data for Hudson 82-001 Leg 2: HUD2.AIR

8000

80

49. Surface seawater $\mathrm{CO}_{2}$ and $\mathrm{N}_{2} \mathrm{O}$ data for Hudson 82-001 Leg 2: HUD2.H2O

50. Atmospheric $\mathrm{CO}_{2}$ and $\mathrm{N}_{2} \mathrm{O}$ data for TTO/TAS Leg 1 :

TAS1_AIR

51. Surface seawater $\mathrm{CO}_{2}$ and $\mathrm{N}_{2} \mathrm{O}$ data for TTO/TAS Leg 1: TAS1.H2O

52. Atmospheric $\mathrm{CO}_{2}$ and $\mathrm{N}_{2} \mathrm{O}$ data for TTO/TAS Leg 2: 
53. Surface seawater $\mathrm{CO}_{2}$ and $\mathrm{N}_{2} \mathrm{O}$ data for TTO/TAS Leg 2: TAS2.H2O

54. Atmospheric $\mathrm{CO}_{2}$ and $\mathrm{N}_{2} \mathrm{O}$ diata for TTO/TAS Leg 3: TAS3.AIR

55. Surface seawater $\mathrm{CO}_{2}$ and $\mathrm{N}_{2} \mathrm{O}$ data for TTO/TAS Leg 3: TAS3.H2O

FB

8000

80

56. Atmospheric $\mathrm{CO}_{2}$ and $\mathrm{N}_{2} \mathrm{O}$ data for Ajax Leg 1:

AJAX1.AIR

8000

80

57. Surface seawater $\mathrm{CO}_{2}$ and $\mathrm{N}_{2} \mathrm{O}$ data for Ajax Leg 1: AJAX1.H2O

58. Atmospheric $\mathrm{CO}_{2}$ and $\mathrm{N}_{2} \mathrm{O}$ data for Ajax Leg 2:

AJAX2.AIR

59. Surface seawater $\mathrm{CO}_{2}$ and $\mathrm{N}_{2} \mathrm{O}$ data for Ajax Leg 2: AJAX2.H2O

60. Atmospheric $\mathrm{CO}_{2}$ and $\mathrm{N}_{2} \mathrm{O}$ data for TPS24 Leg 1:

TPS241.AIR

61. Surface seawater $\mathrm{CO}_{2}$ and $\mathrm{N}_{2} \mathrm{O}$ data for TPS24 Leg 1: TPS241.H2O

62. Atmospheric $\mathrm{CO}$ and $\mathrm{N}_{2} \mathrm{O}$ data for TPS24 Leg 2:

TPS242.AIR

63. Surface seawater $\mathrm{CO}_{2}$ and $\mathrm{N}_{2} \mathrm{O}$ data for TPS24 Leg 2: TPS242.H2O

64. Atmospheric $\mathrm{CO}_{2}$ and $\mathrm{N}_{2} \mathrm{O}$ data for TPS47 Leg 1:

TPS471.AIR

65. Surface seawater $\mathrm{CO}_{2}$ and $\mathrm{N}_{2} \mathrm{O}$ data for TPS47 Leg 1 : TPS471.H2O

66. Atmospheric $\mathrm{CO}_{2}$ and $\mathrm{N}_{2} \mathrm{O}$ data for Ant V Leg 2: 
File number, description, and name
Logical

records
Record

format ${ }^{\circ}$
Block

size
Record

length

67. Surface seawater $\mathrm{CO}_{2}$ and $\mathrm{N}_{2} \mathrm{O}$ data for Ant $\mathrm{V}$ Leg 2: ANT52.H2O

68. Atmospheric $\mathrm{CO}_{2}$ and $\mathrm{N}_{2} \mathrm{O}$ data for Ant V Leg 3: ANT53.AIR

69. Surface seawater $\mathrm{CO}_{2}$ and $\mathrm{N}_{2} \mathrm{O}$ data for Ant $\mathrm{V}$ Leg 3: ANT53.H2O

70. Atmospheric $\mathrm{CO}_{2}$ and $\mathrm{N}_{2} \mathrm{O}$ data for SAVE Transit: SAVEO.AIR

71. Surface seawater $\mathrm{CO}_{2}$ and $\mathrm{N}_{2} \mathrm{O}$ data for SAVE Transit: SAVEO.H2O

72. Atmospheric $\mathrm{CO}_{2}$ and $\mathrm{N}_{2} \mathrm{O}$ data for SAVE Leg 1: SAVE1.AIR

73. Surface seawater $\mathrm{CO}_{2}$ and $\mathrm{N}_{2} \mathrm{O}$ data for SAVE Leg 1: SAVE1.H2O

74. Atmospheric $\mathrm{CO}_{2}$ anci $\mathrm{N}_{2} \mathrm{O}$ data for SAVE Leg 2:

SAVE2.AIR

8000

75. Surface seawater $\mathrm{CO}_{2}$ and $\mathrm{N}_{2} \mathrm{O}$ data for SAVE Leg 2: SAVE2.H2O

76. Atmospheric $\mathrm{CO}_{2}$ and $\mathrm{N}_{2} \mathrm{O}$ data for SAVE Leg 3: SAVE3.AIR

77. Surface seawater $\mathrm{CO}_{2}$ and $\mathrm{N}_{2} \mathrm{O}$ data for SAVE Leg 3: SAVE3.H2O

78. Almospheric $\mathrm{CO}_{2}$ and $\mathrm{N}_{2} \mathrm{O}$ data for SAVE Leg 4:

79. Surface seawater $\mathrm{CO}_{2}$ and $\mathrm{N}_{2} \mathrm{O}$ data for SAVE Leg 4: SÁVE4.H2O

80. Atmospheric $\mathrm{CO}_{2}$ and $\mathrm{N}_{2} \mathrm{O}$ data for SAVE Leg 5: 
File number, description, and name

\section{Logical}

records
Record

format $^{\circ}$
Block

size

Record

length

81. Surface seawater $\mathrm{CO}_{2}$ and

$\mathrm{N}_{2} \mathrm{O}$ data for SAVE Leg 5:

SAVE5.H2O

1927

FB

8000

80

82. Atmospheric $\mathrm{CO}_{2}$ and $\mathrm{N}_{2} \mathrm{O}$ data for SAVE Leg 6:

SAVE6.AIR

1471

FB

8000

80

83. Surface seawater $\mathrm{CO}_{2}$ and $\mathrm{N}_{2} \mathrm{O}$ data for SAVE Leg 6: SAVE6.H2O

FB

8000

80

84. Atmospheric $\mathrm{CO}_{2}$ and $\mathrm{N}_{2} \mathrm{O}$ data for CGC-90 Leg 1:

CGC901.AIR

1175

FB

8000

80

85. Surface seawater $\mathrm{CO}_{2}$ and $\mathrm{N}_{2} \mathrm{O}$ data for CGC-90 Leg 1:

CGC901.H2O

FB

8000

80

86. Atmospheric $\mathrm{CO}_{2}$ and $\mathrm{N}_{2} \mathrm{O}$ data for CGC-90 Leg 2:

CGC902.AIR

996

FB

8000

80

87. Surface seawater $\mathrm{CO}_{2}$ and

$\mathrm{N}_{2} \mathrm{O}$ data for CGC-90 Leg 2 :

CGC902.H2O

1003

FB

8000

80

Total records $1 \overline{102,523} \quad$ (or $\sim 7.1 \mathrm{mB}$ )

${ }^{a} \mathrm{FB}=$ fixed block.

${ }^{b}$ SAS is the registered trademark of SAS Institute, Inc., Cary, NC 27511-8000. 


\section{DESCRIPTIVE FILE ON 'THE TAPE/DISKETTES}

The following is a listing of File 1 on the magnetic tape (or NDP044.DES on the floppy diskettes) distributed by CDIAC. This file is intended to complement the documentation and provide details (i.e., variable descriptions, formats, and units) about the data files on the magnetic tape or floppy diskettes.

\section{TITLE OF THE DATA SET}

Surface Water and Atmospheric Carbon Dioxide and Nitrous Oxide Observations by Shipboard Automated Gas Chromatography: Results from Expeditions between 1977 and 1990.

\section{DATA CONTRIBUTORS}
R. F. Weiss
F. A. Van Woy
P. K. Salameh

Scripps Institution of Oceanography

University of California, San Diego

La Jolla, California

\section{SOURCE AND SCOPE OF THE DATA}

Note: The material provided on the magnetic tape or floppy diskettes for this section is essentially identical to the contents of Sections 4 (Source Information) and 5 (Methodology) of Part I of this documentation.

\section{DATA FORMAT}

Eighty-seven files are provided on this magnetic tape or these floppy diskettes, including (1) this descriptive file - File 1 on the magnetic tape or NDP044.DES on the floppy diskettes; (2) a file containing a list of expedition legs on which measurements were made - File 2 on the magnetic tape or TRACK.LST on the floppy diskettes; (3) a file containing a list of the data filenames corresponding (by number) to the expedition legs listed in File 2 (or TRACK.LST) - File 3 on the magnetic tape or DATA.LST on the floppy diskettes; (4) a FORTRAN-77 retrieval program to read and print any of the data files - File 4 on the magnetic tape or NDP044.FOR on the floppy diskettes; (5) a SAS input/output routine to read and print any of the data files - File 5 on the magnetic tape or NDP044.SAS on the floppy diskettes; and (6)-(87) 82 files containing the surface water and atmospheric $\mathrm{CO}_{2}$ and $\mathrm{N}_{2} \mathrm{O}$ data - Files $6-87$ on the magnetic tape or *.AIR and ${ }^{*} . \mathrm{H} 2 \mathrm{O}$ on the floppy diskettes [with full filenames as listed in File 3 (or DATALST)].

Table 2 (located in the documentation that accompanies this tape/diskettes) presents a partial listing of one of the surface water and atmospheric $\mathrm{CO}_{2}$ and $\mathrm{N}_{2} \mathrm{O}$ data files. The data files are formatted in the following way: 


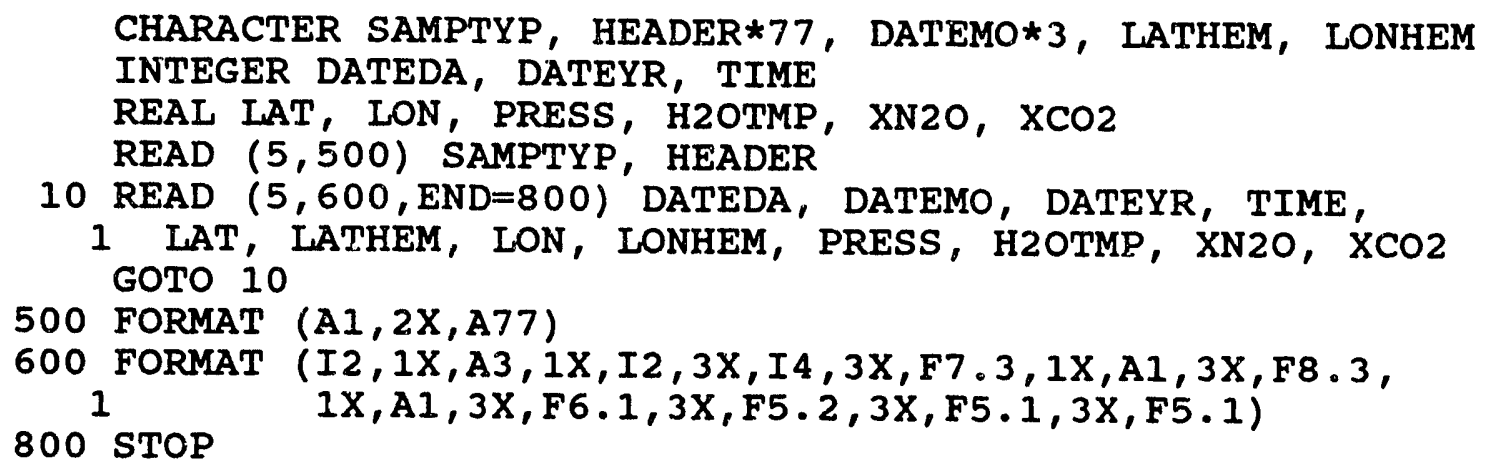

where

SAMPTYP is a one-character code describing the type of :amples being presented in the data file: $\mathrm{A}=$ atmospheric samples, $\mathrm{S}=$ surface seawater (i.e., gas equilibrated with surface seawater) samples;

HEADER is a descriptive character string consisting of (1) the name of the expedition (e.g., AJAX Leg 1) and (2) the name of the research vessel (e.g., R/V Knorr);

DATEDA is the numeric day of the month on which the sample was coilected;

DATEMO is the three-letter abbreviation (Jan, Feb, etc.) for the month in which the sample was collected;

DATEYR is the final two digits of the year (since 1900) in which the sample was collected;

TIME is the Greenwich Mean Time at which the sample was collected, expressed in 24-hour time from 0000 to 2359 ;

LAT is the latitude (in decimal degrees) at which the sample was collected, with possible values from -90.000 to 90.000 (north latitudes are represented as positive);

LATHEM is the latitudinal hemisphere in which the sample was taken: $\mathrm{N}=$ Northern Hemisphere, $\mathrm{S}=$ Southern Hemisphere;

LON is the longitude (in decimal degrees) at which the sample was collected, with possible values from -180.000 to 180.000 (east longitudes are represented as positive);

LONHEM is the longitudinal hemisphere in which the sample was taken: $\mathrm{E}=$ Eastern Hemisphere, $\mathrm{W}=$ Western Hemisphere; 
PRESS is the approximate sea level barometric pressure in mBar, interpolated from discrete values recorded on the ship at hydrographic stations;

H2OTMP is the approximate surface water temperature in degrees Celsius, interpolated from discrete values recorded on the ship at hydrographic stations;

$\mathrm{XN2O}$ is the dry gas mole fraction of nitrous oxide $\left(\mathrm{N}_{2} \mathrm{O}\right)$ in the sample, measured in parts per billion (ppb);

$\mathrm{XCO} 2$ is the dry gas mole fraction of carbon dioxide $\left(\mathrm{CO}_{2}\right)$ in the sample, measured in parts per million (ppm);

Stated in tabular form the contents include the following:

\begin{tabular}{|c|c|c|c|c|c|}
\hline Variable $^{a}$ & $\begin{array}{l}\text { Variable } \\
\text { type }\end{array}$ & $\begin{array}{l}\text { Variable } \\
\text { width }^{b}\end{array}$ & Line & $\begin{array}{l}\text { Starting } \\
\text { column }\end{array}$ & $\begin{array}{l}\text { Ending } \\
\text { column }\end{array}$ \\
\hline SAMPTYYP & Character & Al & 1 & 1 & 1 \\
\hline HEADER & Character & A77 & 1 & 4 & 80 \\
\hline DATEDA & Numeric & 12 & $2 \ldots n$ & 1 & 2 \\
\hline DATEMO & Character & $\mathrm{A}^{3}$ & $2 \ldots n$ & 4 & 6 \\
\hline DATEYR & Numeric & 12 & $2 \ldots n$ & 8 & 9 \\
\hline TIME & Numeric & 14 & $2 \ldots n$ & 13 & 16 \\
\hline LAT & Numeric & F7.3 & $2 \ldots n$ & 20 & 26 \\
\hline LATHEM & Character & A1 & $2 \ldots n$ & 28 & 28 \\
\hline LON & Numeric & F8.3 & $2 \ldots n$ & 32 & 39 \\
\hline LONHHEM & Characte: & A1 & $2 \ldots n$ & 41 & 41 \\
\hline PRESS & Numeric & F6.1 & $2 \ldots n$ & 45 & 50 \\
\hline H2OTMP & Numeric & F5.2 & $2 \ldots n$ & 54 & 58 \\
\hline $\mathrm{XN2O}$ & Numeric & F5.1 & $2 \ldots n$ & 62 & 66 \\
\hline $\mathrm{XCO} 2$ & Numeric & F5.1 & $2 \ldots n$ & 70 & 74 \\
\hline
\end{tabular}

${ }^{a}$ Missing values are represented as follows - PRESS: -999.9; H2OTMP: 99.99; XN2O: -99.9; XCO2: -99.9.

${ }^{b}$ Values for variable width are entered as FORTRAN 77 format codes. 
Table 2. Partial listing of one of the surface water and atmospheric $\mathrm{CO}_{2}$ and $\mathrm{N}_{2} \mathrm{O}$ data files (File 23 on the magnetic tape or NORPAX0.H2O on the floppy diskettes)

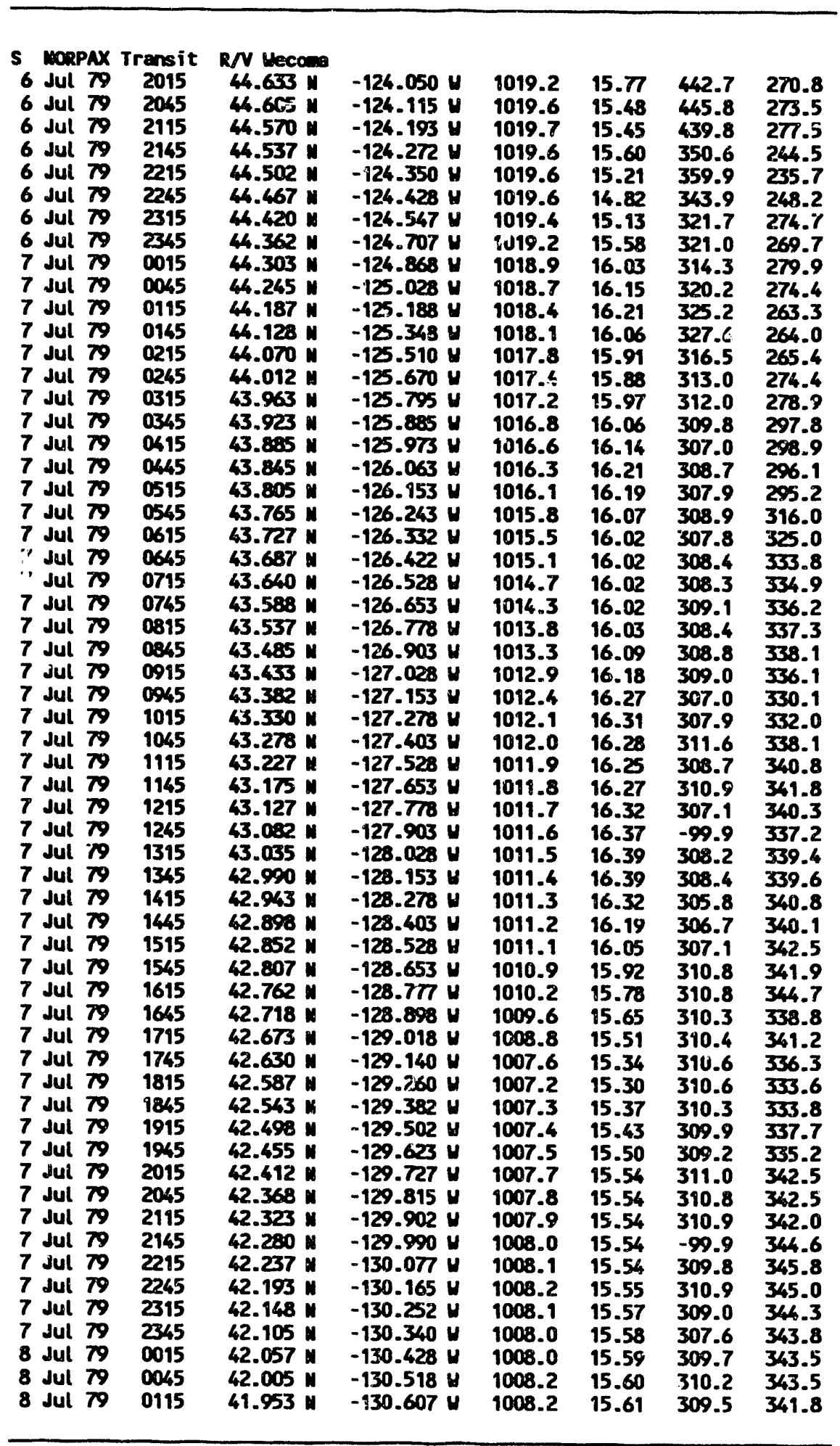




\section{REFERENCES}

Note: The material provided on the magnetic tape or floppy diskettes for this section is identical to the contents of Section 9 (References) of Part I of this documentation. 


\section{LISTING OF THE FORTRAN-77 DATA RETRIEVAL PROGRAM}

The following is a listing of the FORTRAN-77 data retrieval program (File 4 on magnetic tape or NDP044.FOR on floppy diskette) provided by CDIAC to read and print the surface water and atmospheric $\mathrm{CO}_{2}$ and $\mathrm{N}_{2} \mathrm{O}$ data (Files $6-87$ on magnetic tape or files *.AIR and *.H2O on floppy diskettes). The job control language (JCL) statements (preceded by $/ /$ or $/ *$ ) shown below are not provided in the file on the tape/diskette;

requestors must add JCL statements themselves if required. The statements required will vary for each operating system. The JCL statements shown below are provided to illustrate the statements that an individual using an IBM mainframe (e.g., IBM 3090) at ORNL would need to read these data from a nine-track, $6250 \mathrm{BPI}$, standard-labeled tape with characters written in EBCDIC.

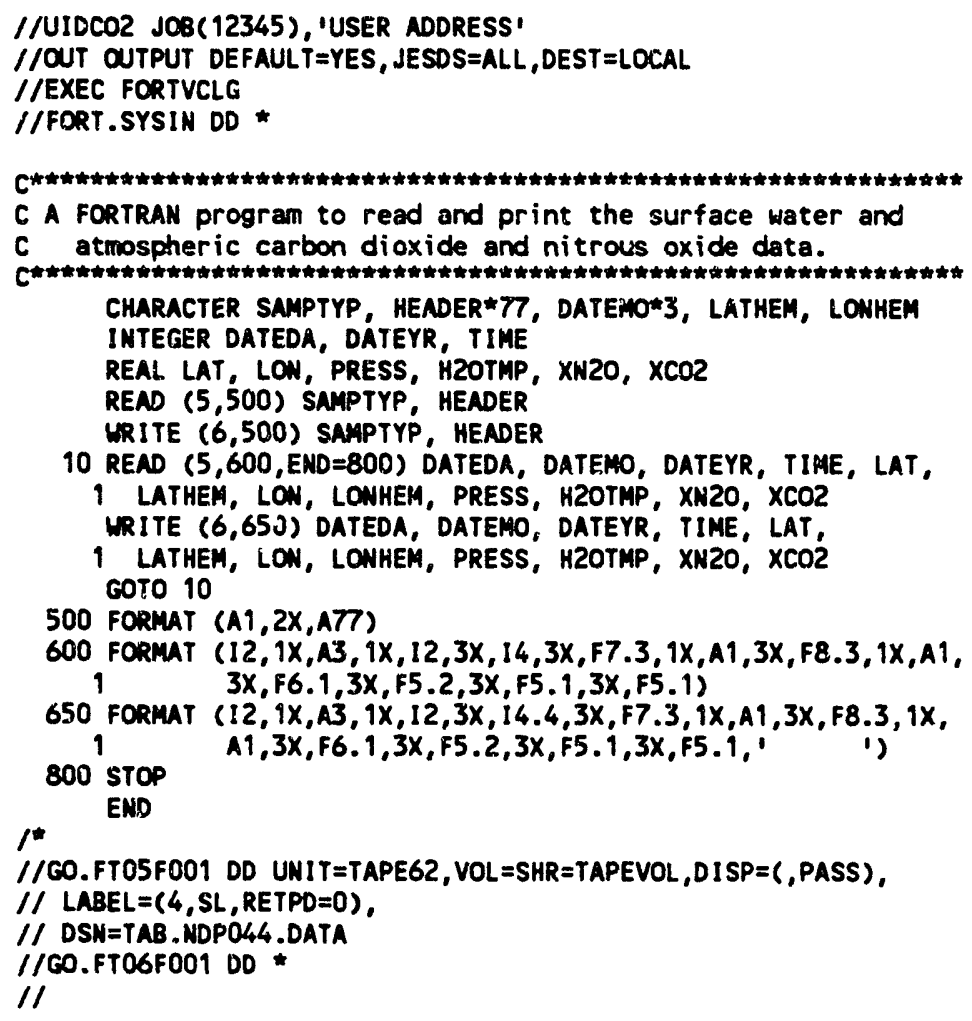




\section{LISTING OF THE SAS INPUT/OUTPUT RETRIEVAL PROGRAM}

The following is a listing of the SAS data retrieval program (File 5 on magnetic tape or NDP044.SAS on floppy diskette) provided by CDIAC to read and print the surface water and atmospheric $\mathrm{CO}_{2}$ and $\mathrm{N}_{2} \mathrm{O}$ data (Files 6-87 on magnetic tape or files *.AIR and *.H2O on floppy diskettes). The JCL statements (preceded by $/ /$ or $/ *$ ) shown below are not provided in the file on the tape/diskette; requestors must add JCL statements themselves if required. The statements required will vary for each operating system. The JCL statements shown below are provided to illustrate the statements that an individual using an IBM mainframe (e.g., IBM 3090) at ORNL would need to read these data from a nine-track, $6250 \mathrm{BPI}$, standard-labeled tape with characters written in EBCDIC.

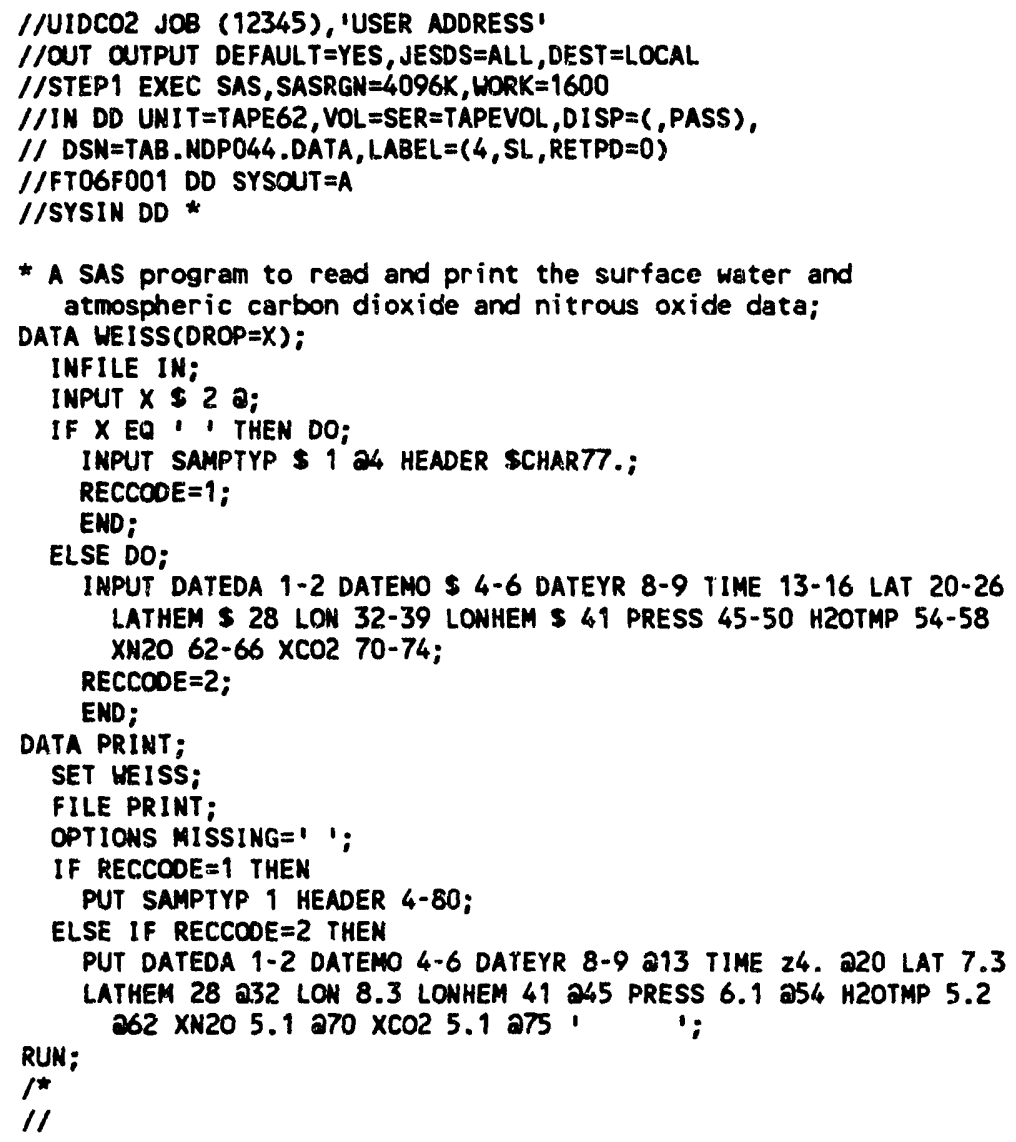

a SAS is the registered trademark of SAS Institute, Inc., Cary, NC 27511-8000. 


\section{VERIFICATION OF DATA TRANSPORT}

The surface water and atmospheric $\mathrm{CO}_{2}$ and $\mathrm{N}_{2} \mathrm{O}$ data can be read by using the FORTRAN or SAS input/output routines provided. Users should verify that the data file has been correctly transported to their systems by generating some or all of the statistics presented in Table 3. These statistics were generated in FORTRAN but can be duplicated in other languages or statistical packages. If the statistics generated by the user differ from those presented here, the data files may have been corrupted in transport.

These statistics are presented only as a tool to ensure proper reading of the data files. They are not to be construed as summarizing the surface water and atmospheric $\mathrm{CO}_{2}$ and $\mathrm{N}_{2} \mathrm{O}$ data. 
Table 3. Characteristics of numeric variables in the collective surface water and atmospheric $\mathrm{CO}_{2}$ and $\mathrm{N}_{2} \mathrm{O}$ data files

\begin{tabular}{lcrrr}
\hline & $\begin{array}{c}\text { Number } \\
\text { of } \\
\text { Variable }\end{array}$ & Mean & $\begin{array}{c}\text { Minimum } \\
\text { value }\end{array}$ & $\begin{array}{c}\text { Maximum } \\
\text { value }\end{array}$ \\
\hline DATEDA & 101920 & 14.991 & 1.000 & 31.000 \\
DATEYR & 101920 & 83.469 & 77.000 & 90.000 \\
TIME & 101920 & 1176.071 & 0.000 & 2359.000 \\
LAT & 101920 & -2.444 & -77.177 & 79.005 \\
LON & 101920 & -36.570 & -179.998 & 179.998 \\
PRESS & 101920 & 843.456 & -999.900 & 1035.400 \\
H2OTMP & 101920 & 18.075 & -2.130 & 99.990 \\
XN2O & 101920 & 279.027 & -99.900 & 725.600 \\
XCO2 & 101920 & 291.289 & -99.900 & 737.900 \\
\hline
\end{tabular}

The following is a listing of the FORTRAN program used to generate the statistics described in Table 3:

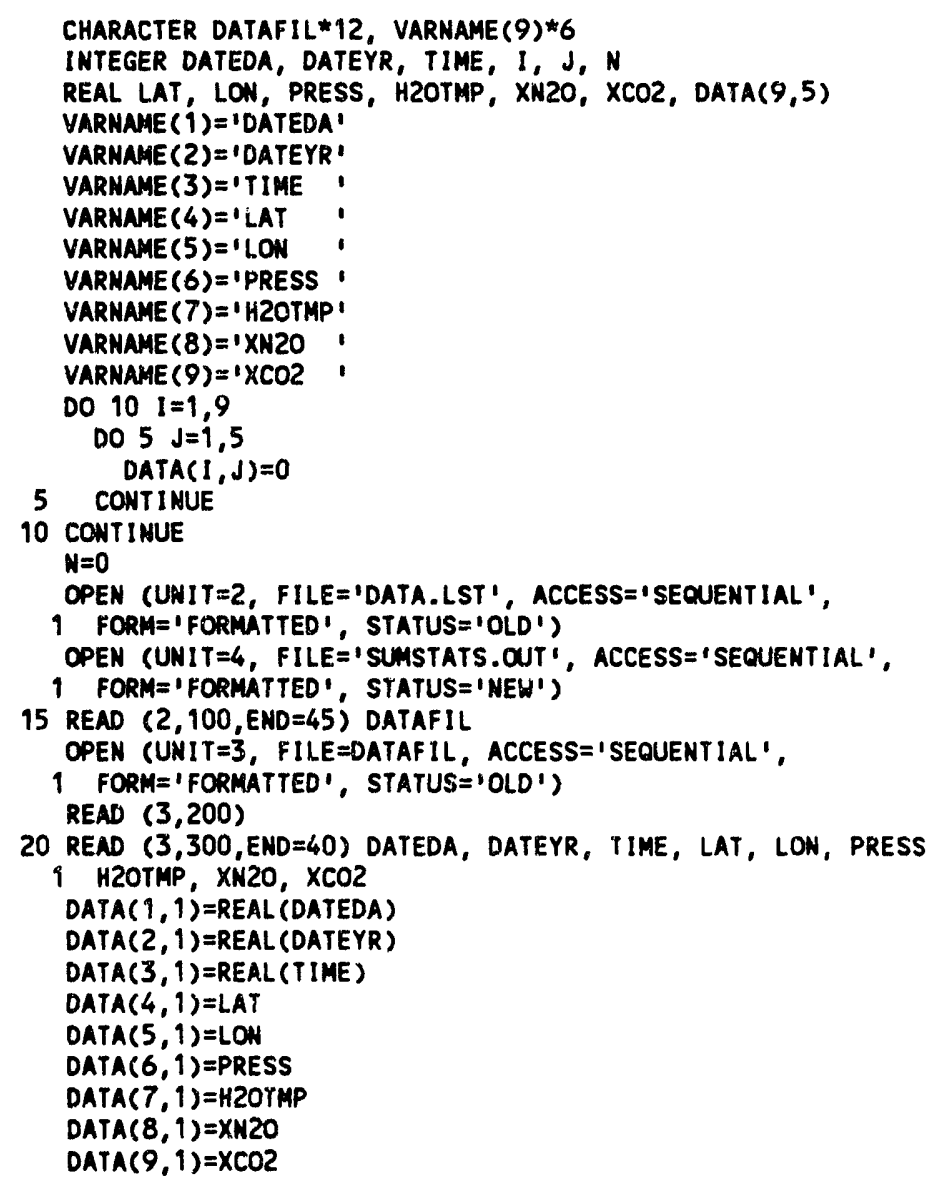



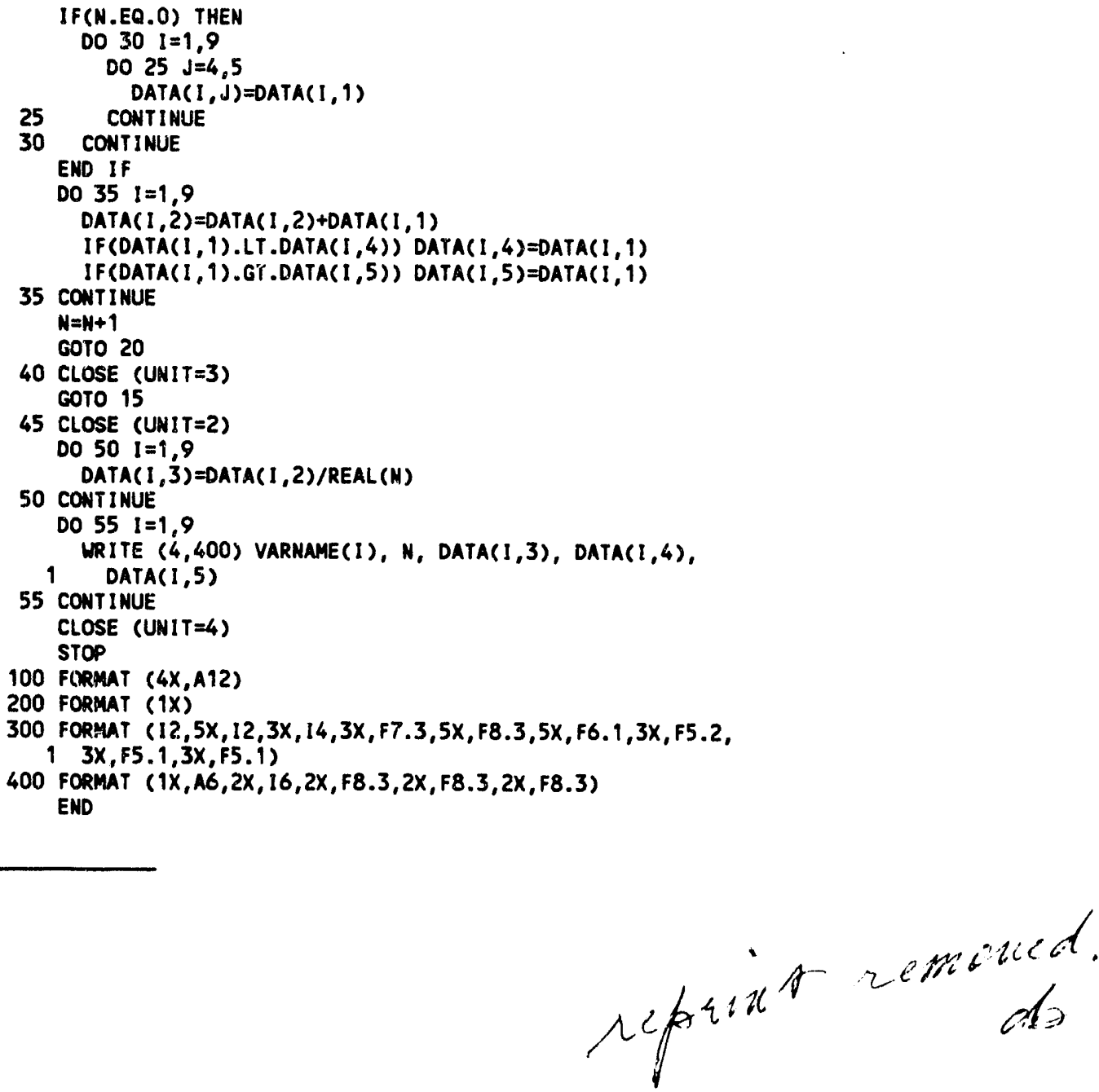
SIO 92-11

ORNL/CDIAC-59

NDP-044

\title{
INTERNAL DISTRIBUTION
}

\author{
1. T. A. Boden \\ 2. J. B. Cannon \\ 3. J. H. Cushman \\ 4. R. M. Cushman \\ 5. M. P. Farrell \\ 6. D. E. Fowler \\ 7. C. W. Gehrs \\ 8. S. G. Hildebrand \\ 9. P. Kanciruk \\ 10. T. H. Peng \\ 11. D. E. Reichle \\ 12. R. J. Sepanski \\ 13. F. E. Sharples \\ 14. D. S. Shriner \\ 16. S. H. Stow \\ 17. R. I. Van Hook \\ 318. Central Research Library \\ 319-323. ESD Library \\ 324. Information Analysis Library \\ 325-326. Laboratory Records Department \\ 327. Laboratory Records, RC \\ 328. ORNL Patent Office \\ 329. ORNL Y-12 Technical Library
}

18-317. CDIAC

\section{EXTERNAL DISTRIBUTION}

330. S. S. Alexander, Pennsylvania State University, Department of Geosciences, 503 Deike Building, University Park, PA 16802

331. J. H. Allen, National Oceanic and Atmospheric Administration, National Geophysical Data Center Code E/GC2, 325 Broadway, Boulder, CO 80303

332. D. Alvic, EERC/UT, Pellissippi Office, Ste. 100, 10521 Research Drive, Knoxville, TN 37932

333. N. Andersen, National Science Foundation, OCE, 1800 G. Street, Washington, DC 20550

334. R. C. Barry, University of Colorado, World Data Center A, Glaciology, CIRES, 
Campus Box 449, Boulder, CO 80309-0449

335. P. Brewer, Monterey Bay Aquariam Res. Inst., 160 Central Avenue, Pacific Grove, CA 93950

336. M. A. Chinnery, National Oceanic and Atmospheric Administration, National Geophysical Data Center Code E/GC2, 325 Broadway, Boulder, CO 80303

337. Roger C. Dahlman, Global Change Research Program, Environmental Sciences Division, Office of Health and Environmental Research, ER-74, U.S. Department of Energy, Washington, DC 20585

338. W. Draeger, EROS Data Center, U.S. Geological Survey, Sioux Falls, SD 57198

339. M. Dryer, National Oceanic and Atmospheric Administration, Space Environmental Lab., ERL/OAR, R/E/SE, 320 Broadway, Boulder, CO 80303

340. R. Feely, National Oceanic and Atm. Adm., Pacific Marine and Enviro. Lab, 7600 Sand Point Way, NE, Seattle, WA 98115

341. J. Filson, National Earthquake Information Center, U.S. Geological Survey, Denver Federal Center, P.O. Box 20546, Denver, CO 80225

342. Jerry F. Franklin, Bloedel Professor of Ecosystem Analysis, College of Forest Resources, University of Washington, Anderson Hall (AR-10), Seattle, WA 98195

343. David J. Galas, Office of Health and Environmental Research, ER-70, U.S. Department of Energy, Washington, DC 20585

344. S. Graves, National Aeronautics and Space Administration Headquarters Code SED, 600 Independence Avenue, Washington, DC 20546

345. J. L. Green, National Space Science Data Center, NASA Goddard Space Flight Center, Code 630.2, Greenbelt, MD 20771

346. Thomas J. Gross, Global Change Research Program, Environmental Sciences Division, Office of Health and Environmental Research, ER-74, U.S. Department of Energy, Washington, DC 20585

347. K D. Hadeen, National Oceanic and Atmospheric Administration, NESDIS/NCDC, Federal Building MC E/CC, Asheville, NC 28801

348. R. C. Harriss, Institute for the Study of Earth, Oceans, and Space, Science and Engineering Research Building, University of New Hampshire, Durham, NH 03824

349-353. G. Heimerdinger, Woods Hole Oceanographic Institution, McLean Building, Woods Hole, MA 02543 
354. W. J. Hinze, Purdue University, Department of Earth and Atmospheric Sciences, West Lafayette, IN 47907

355. G. Y. Jordy, Director, Office of Program Analysis, Office of Energy Research, ER-30, G-226, U.S. Department of Energy, Washington, DC 20585

356. D. Lauer, EROS Data Center, U.S. Geological Survey, Sioux Falls, SD 57198

357. S. Levitus, NOAA/National Oceanographic Data Center, 1825 Connecticut Avenue, NW, Washington, DC 20235

358. M. S. Loughridge, National Oceanic and Atmospheric Administration, National Geophysical Data Center, Code E/GC3, 325 Broadway, Boulder, CO 80303

359. H. M. McCammon, Acting Deputy Director, Environmental Sciences Division, Office of Health and Environmental Research, Office of Energy Research, ER-74, U.S. Department of Energy, Washington, DC 20585

360. L. Merlivat, Universite Pierre et Marie Curie, LODYC, 2 Etage, 4 place Jussieu, 75252 Paris Cedex 05

361. R. H. Olsen, Vice President for Research, University of Michigan, Medical Science Building II, \#5605, 1301 East Catherine Street, Ann Arbor, MI 48109-0620

362. J. T. Overpeck, National Oceanic and Atmospheric Administration, National Geophysical Data Center, Paleoclimatology Program, 325 Broadway E/EC, Boulder, CO 80303

363. Ari Patrinos, Director, Environmental Sciences Division, Office of Health and Environmental Research, ER-74, U.S. Department of Energ, Washington, DC 20585

364. S. Ichtiaque Rasool, IGBP Data and Information System Office, Universite Paris, Tour 26, 4 Etage, Aile 26-16, 4 Place Jussieu, 75230 Paris, Cedex 06, France

365. Michael R. Riches, Global Change Research Program, Environmental Sciences Division, Office of Health and Environmental Research. ER-74, U.S. Department of Energy, Washington, DC 20585

366. S. Ruttenberg, Univ. Corporation for Atmospheric Research, CSNET, P. O. Box 3000, Boulder, CO 80307-3000

367-371. Peter K. Salameh, Scripps Institution of Oceanography, 8602 La Jolla Shores Drive, La Jolla, CA 92037

372. A. L. Shumbera, National Oceanic and Atmospheric Administration, WDC-A for Meteorology, National Climatic Data Center, Federal Building MC E/CC, Asheville, NC 28801 
373-377. F. A. Van Woy, Scripps Institution of Oceanography, 8602 La Jolla Shores Drive, La Jolla, CA 92037

378-382. SIO Library, 8602 La Jolla Shores Drive, La Jolla, CA 92037

383-389. SIO Reference Series Contribution, 8602 La Jolla Shores Drive, La Jolla, CA 92037

390-391. SIO Technical Publications, 8602 La Jolla Shores, La Jolla, CA 92037

392. T. Takahashi, Columbia University, Lamont-Doherty Geological Obs., Palisades, NY 10964

393. P. Tans, National Oceanic and Atm. Adm., Mailcode R/E/CG1, 325 Broadway, Boulder, CO 80304

394. F. Webster, University of Delaware, College of Marine Studies, Lewes, DE 19958

395-409. Ray F. Weiss, Scripps Institution of Oceanography, 2271 Ritter Hall, 8602 I.a Jo!la Shores Drive, La Jolla, CA 92037

410. F. J. Wobber, Environmental Sciences Division, Office of Health and Environmental Research, Office of Energy Research, ER-74, U.S. Department of Energy, Washington, DC 20585

411. L. W. Wolf, National Research Council, Commission on Geosciences, Environment, and Resources, 2101 Constitution Avenue, Washington, DC 20418

412. Office of Assistant Manager for Energy Research and Development, U.S. Department of Energy Oak Ridge Field Office, P. O. Box 2001, Oak Ridge, TN 37831-8600

413-414. Office of Scientific and Technical Information, P. O. Box 62, Oak Ridge, TN 37831 
DATE FILMED $5 / 5 / 93$ 
" 